Hune 12, 1997 ENGINEERING Data tRansmitTAL

$\sin 22$ 27

Page 1 of 1

DCL $B M W$ Q/ISITT

2. To: (Receiving Organization)

Engineering Administration

3. From: (Originating Organization) FFTF ASEE 18240

5. Proi./Prog./Dept./Div.:

F-031

6. Design Authority/ Design Agent/Cog. Engr, :

Derek Jones

B. Originator Remarks:

Ladder Logic Diagrams for the Sodium Storage Facility PLC's

4. Related EDT NO.:

7. Purchase Order No.: $N / A$

9. Equip./Component No.: C-3005

10. System/BIdg./Facility: S93B/402/SSF

11. Receiver Renarks: 11A. Design Baseline Document? [X] Yes [] No

12. Major Assm. Dwg. No.: $\mathrm{H}-4-302426$

13. Permit/Permit Application No.:

14. Required Response Date:

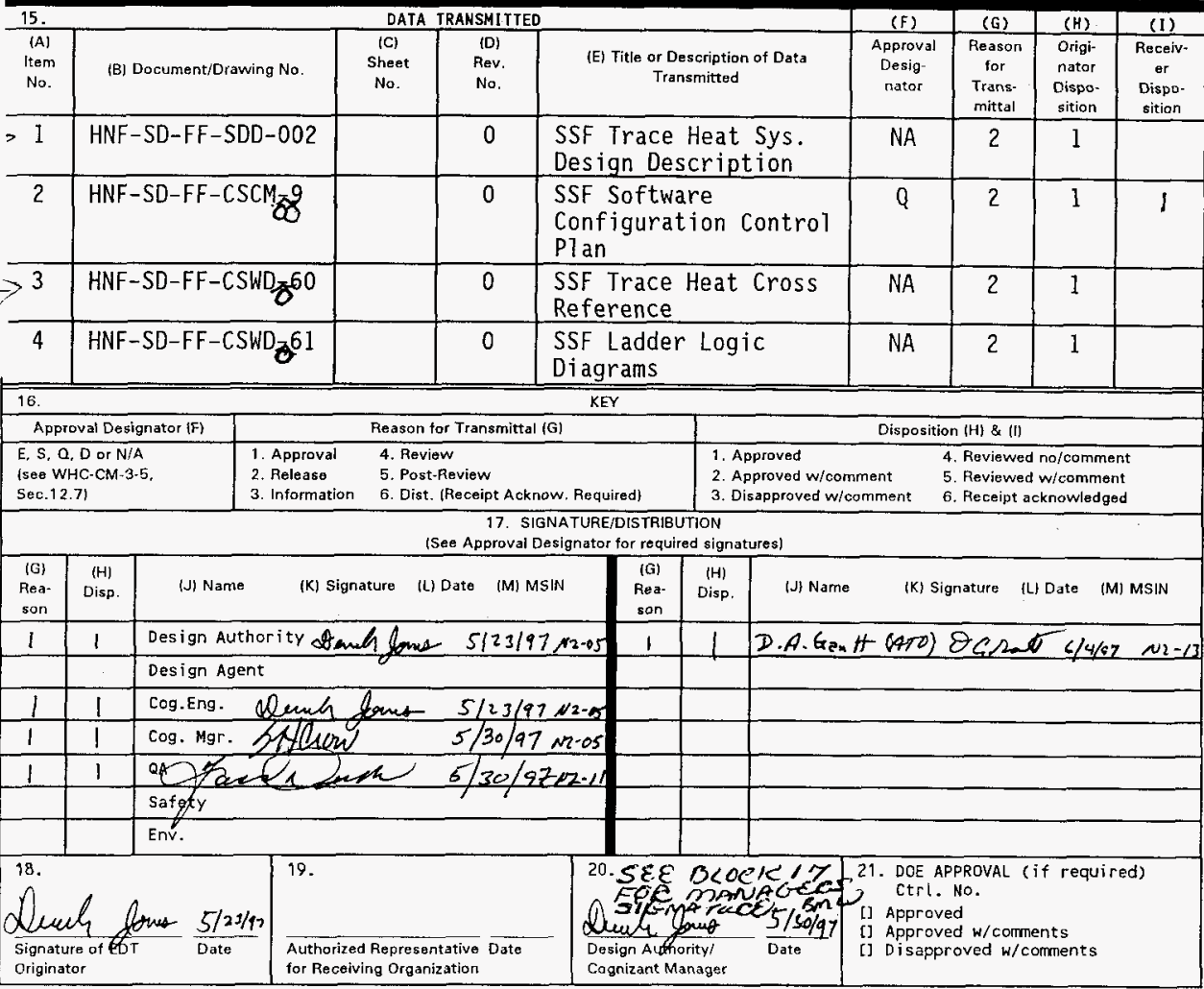

BD - 7400-172-2 (05/96) GEF097 
THIS PAGE INTENTIONALLY L...T BLANK 


\title{
Sodium Storage Facility Trace Heat Cross Reference
}

Derek D. Jones

B \& W Hanford Company, Richland, WA 99352

U.S. Department of Energy Contract DE-AC06-96RL13200

\author{
EDT/ECN: $619594 \quad$ UC: 541 \\ Org Code: 18240 Charge Code: E29146/B1/82 \\ B\&R Code: EX7002000 Total Pages: 209
}

Key Words: Sodium, SSF, Trace Heat, PLC, Computer

Abstract: This document provides the computer technician or software engineer with a method to correlate the PLC ladder logic rung numbers with the device tag in the man machine interface software.

TRADEMARK DISCLAIMER. Reference herein to any specific comercial product, process, or service by trade name, trademark, manufacturer, or otherwise, does not necessarily constitute or imply its endorsement, recommendation, or favoring by the United States Government or any agency thereof or its contractors or subcontractors.

Printed in the United States of America. To obtain copies of this document, contact: Document Control Services, P.O. Box 950, Mailstop H6-08, Richland WA 99352, Phone (509) 372-2420;

Fax $(509) 376-4989$.
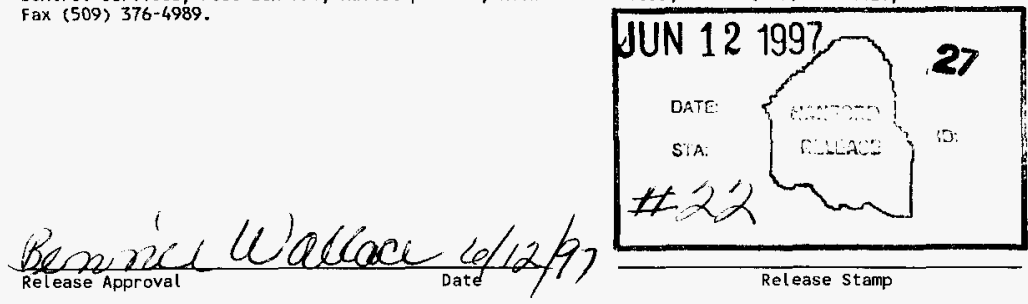

\section{Approved for Public Release}


THIS PAGE INTENTIONALLY LEFT BLANK 


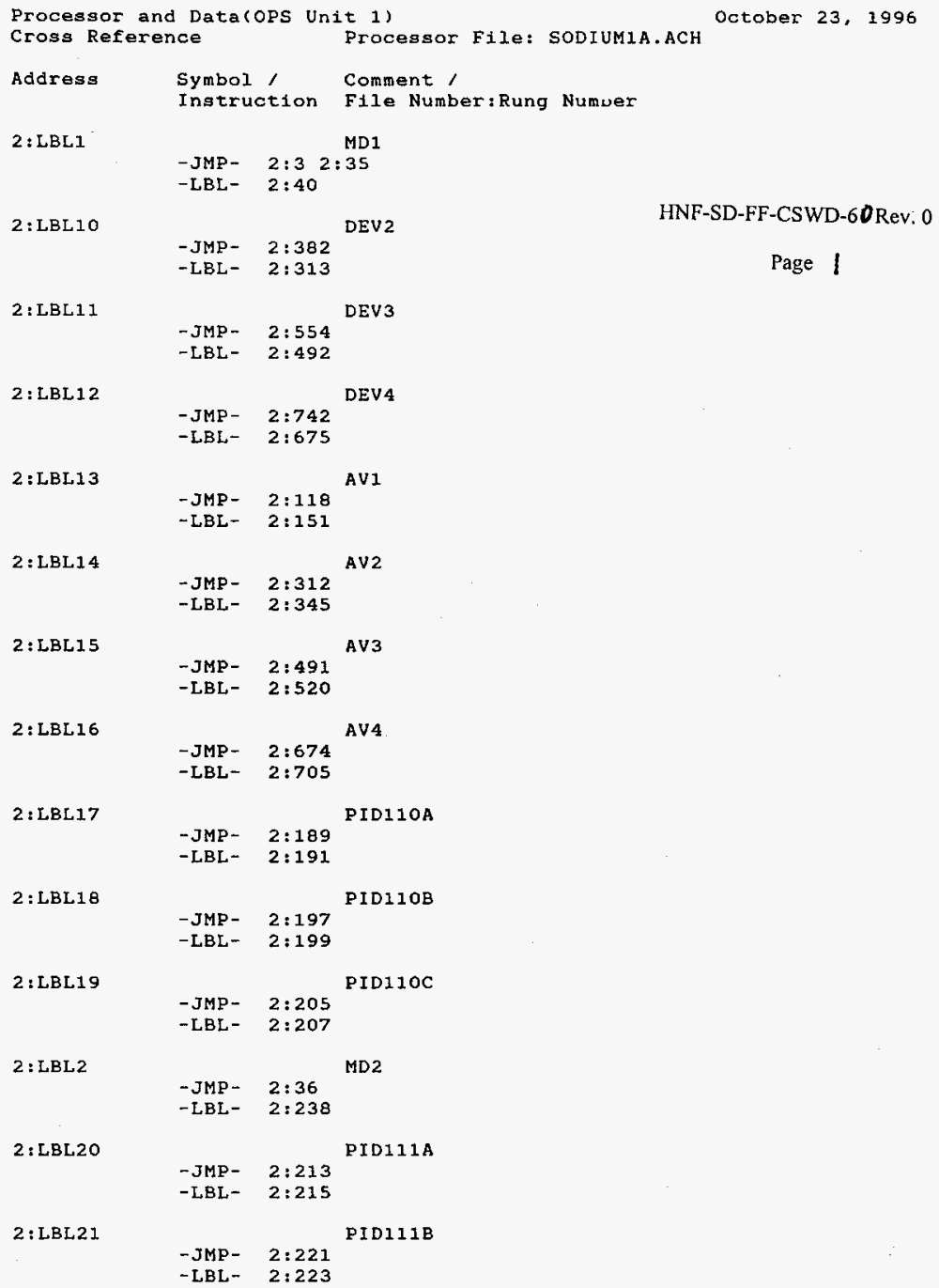

HNF-SD-FF-CSWD-60Rev. 0

\section{Page 1}




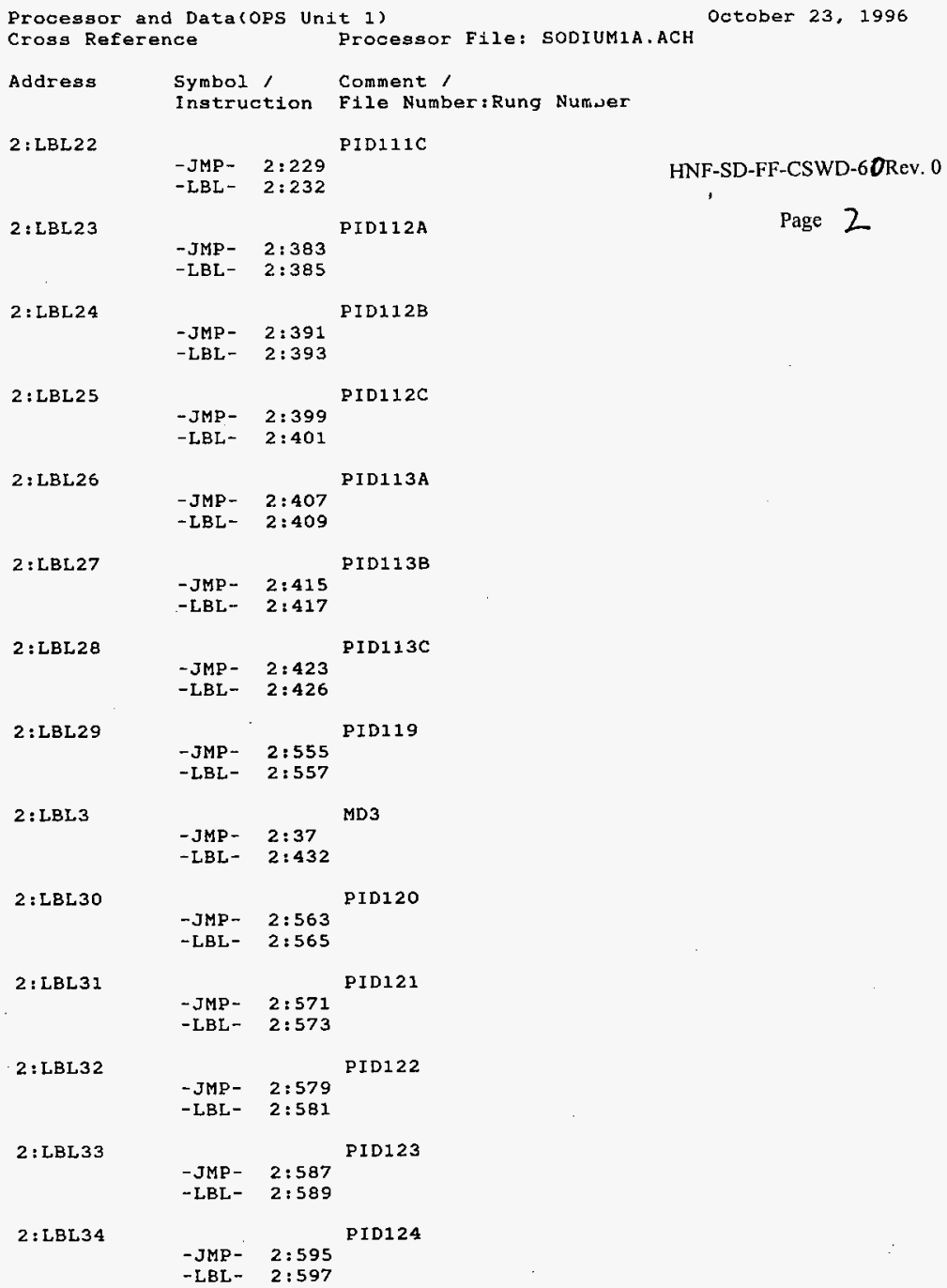

PID122

PID123

PID124

HNF-SD-FF-CSWD-60Rev. 0

PID112B

PID113A

PID113C

MD3

PID120

PID121 


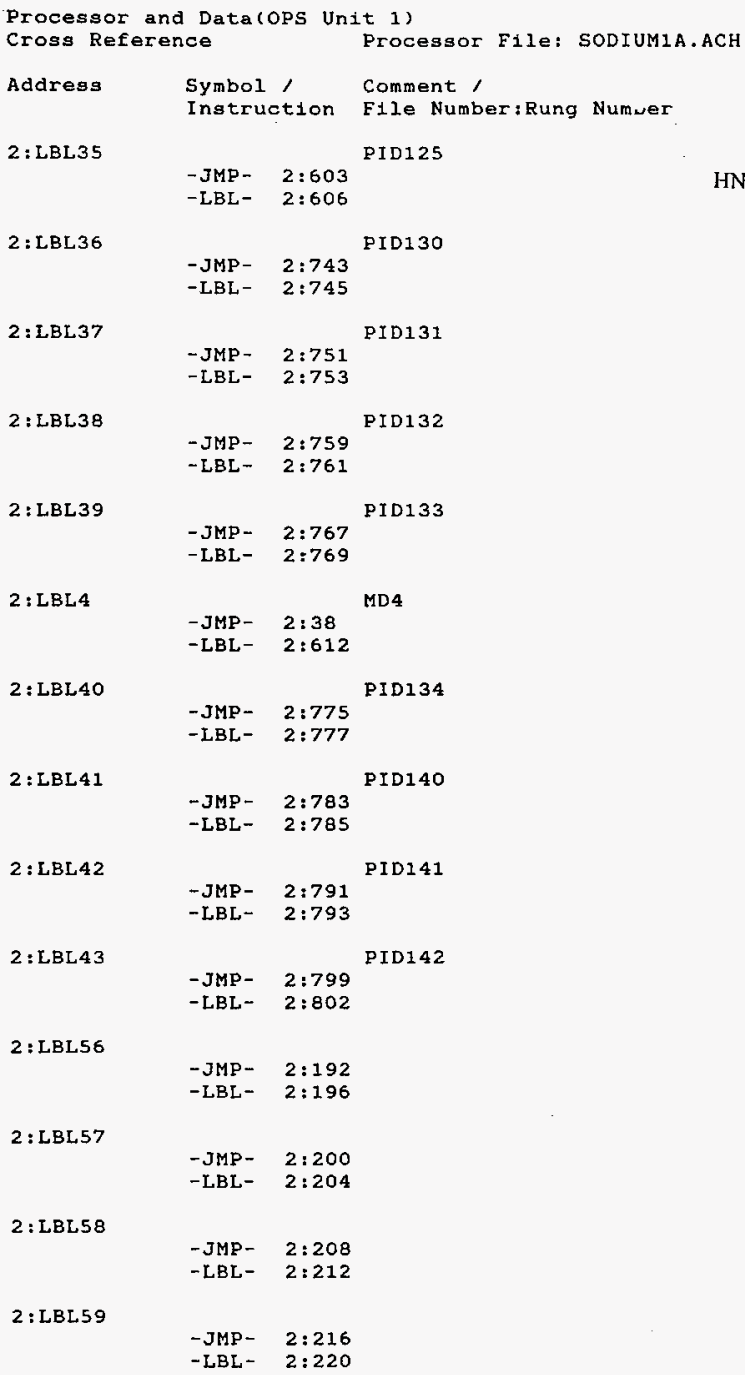


Processor and Data(OPS Unit 1) October 23, 1996 Page. Cross Reference Processor File: SODIUMiA.ACH

Address Symbol $/$ Comment,

Instruction File Number:Rung Numuer

2 : LBL6

NOT END

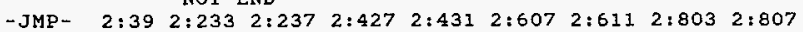

$-\mathrm{LBL}-\quad 2: 808$

$2:$ LBL6O

- JMP - $2: 224$

- LBL- $2: 228$

HNF-SD-FF-CSWD- 6 DRev. 0

$2:$ LBL62

- JMP- $\quad 2: 394$

Page 4

-LBL- $2: 398$

2 : LBL 63

-JMP- $2: 402$

-LBL- 2:406

2: LBL64

-JMP- $2: 410$

-LBL- $2: 414$

$2:$ LBL 65

-JMP- $2: 418$

-LBL- $2: 422$

$2:$ LBL 67

-JMP- 2:558

-LBL- 2:562

2 : LBL 68

- JMP - 2:566

-LBL- 2:570

2:LBL69

-JMP- 2:574

-LBL- $2: 578$

2:LBL7

-JMP - 2:31

-LBL- $2: 909$

$2: \operatorname{LBL} 70$

- JMP- 2:582

-LBL- $2: 586$

2: LBL7 1

- JMP - 2:590

-LBL- 2:594

2: LBL72

-JMP - $\quad 2: 598$

-LBL- $2: 602$

$2:$ LBL 74

- JMP- 2:746

-LBL- $2: 750$ 


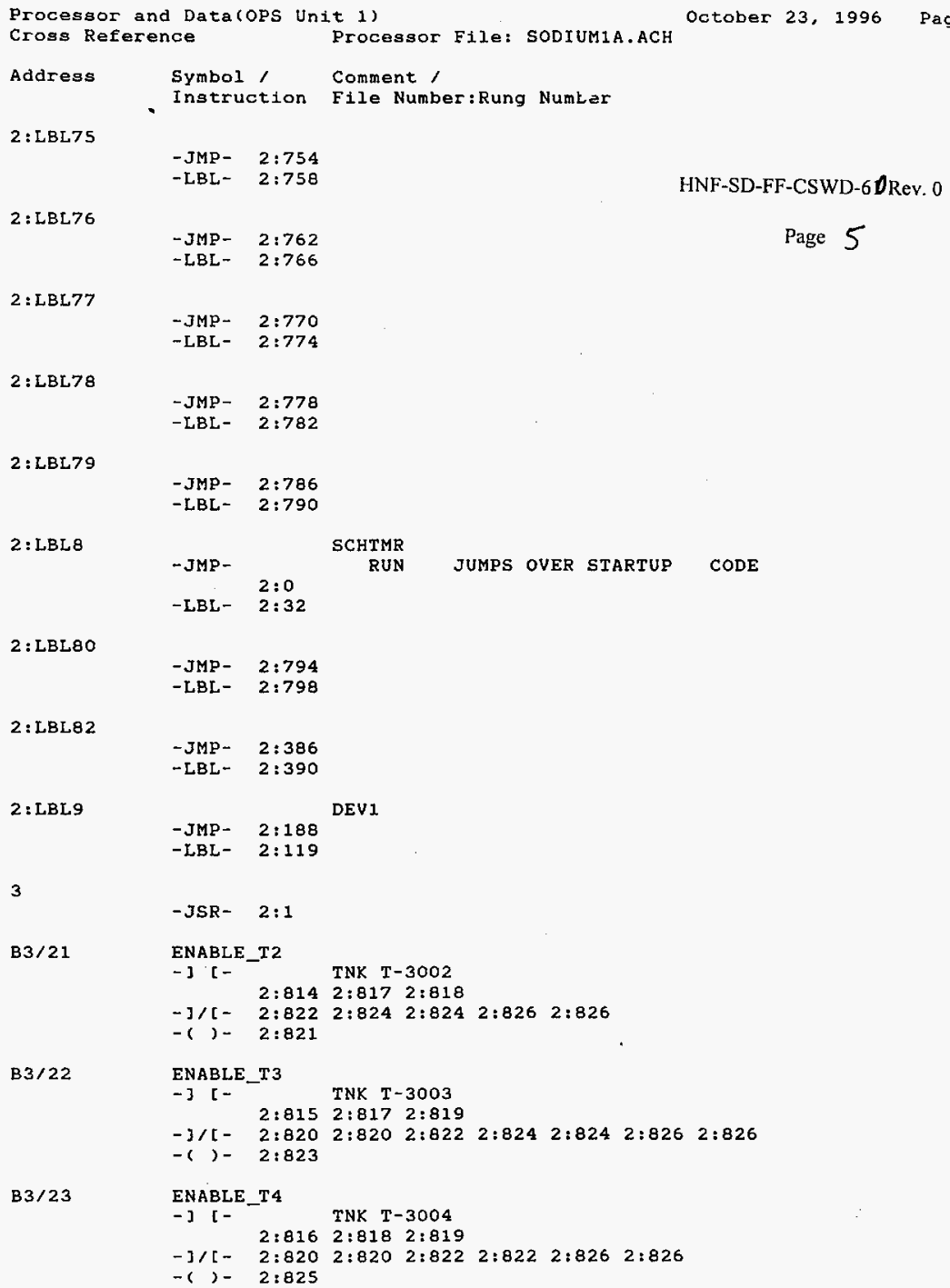


Processor and Data(OPS Unit 1)

Cross Reference Processor File: SODIUMIA.ACH

October 23, 1996 Page 6

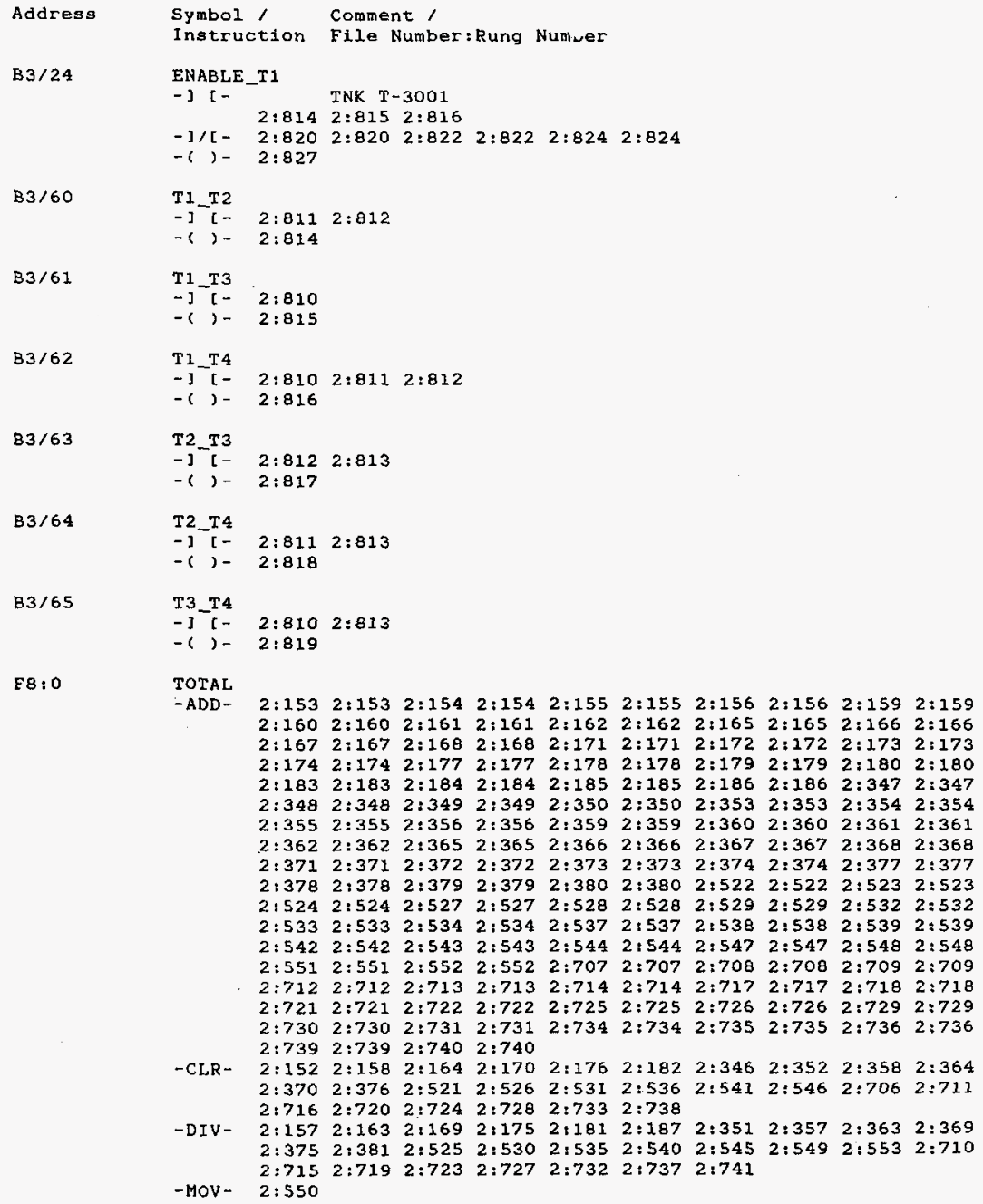

HNF-SD-FF-CSWD-6D Rev. 0

Page 6 


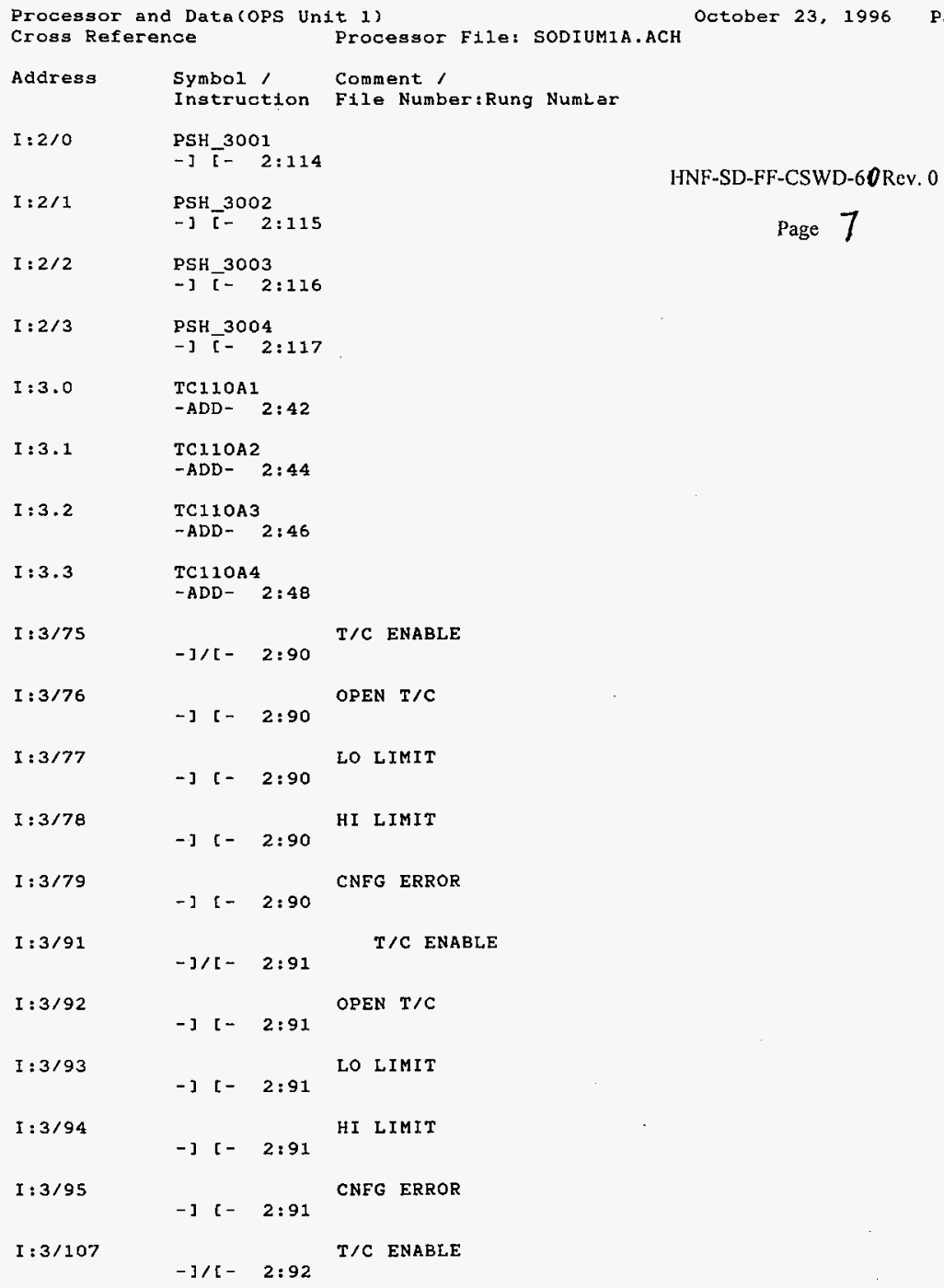

HNF-SD-FF-CSWD-60Rev. 0 


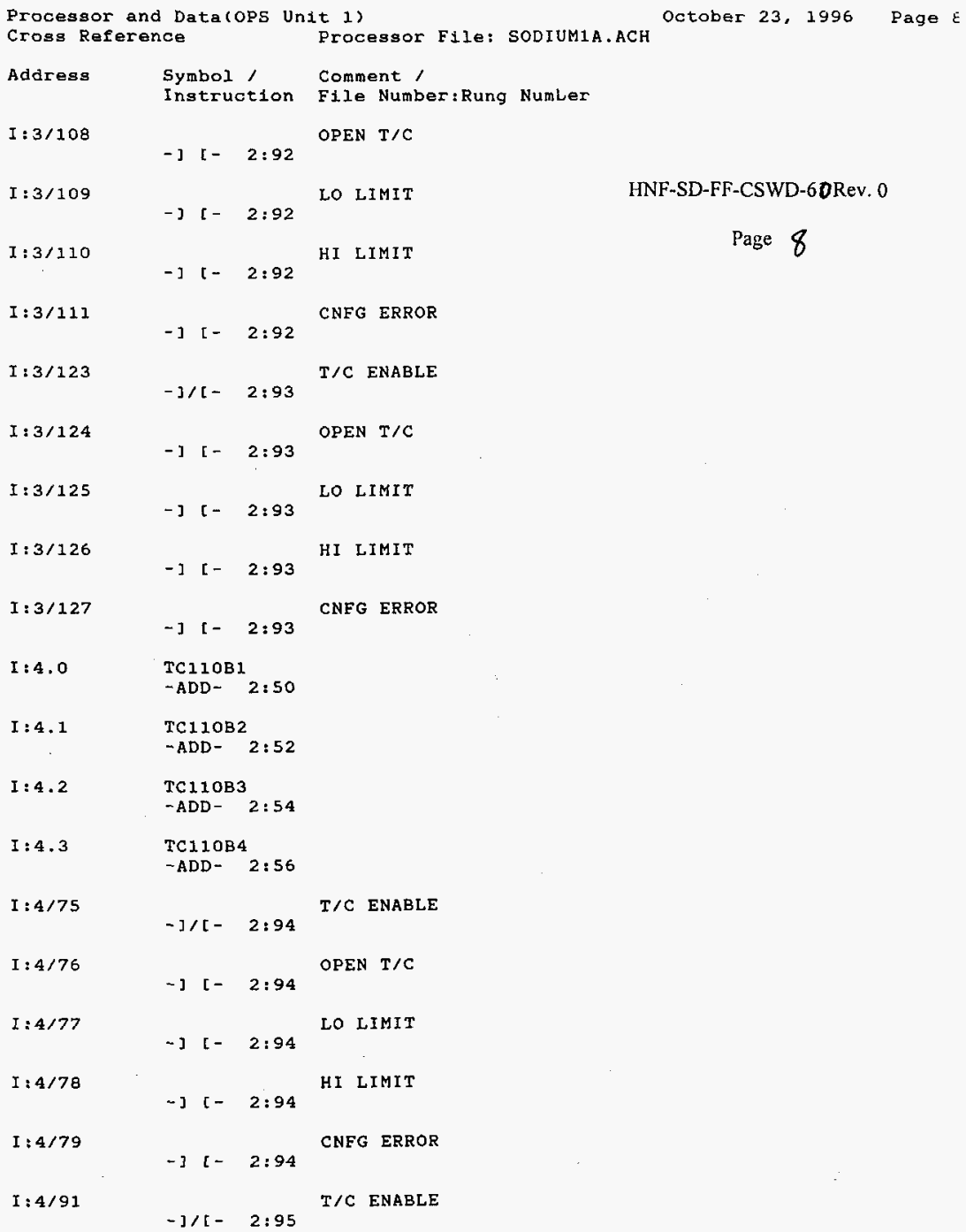




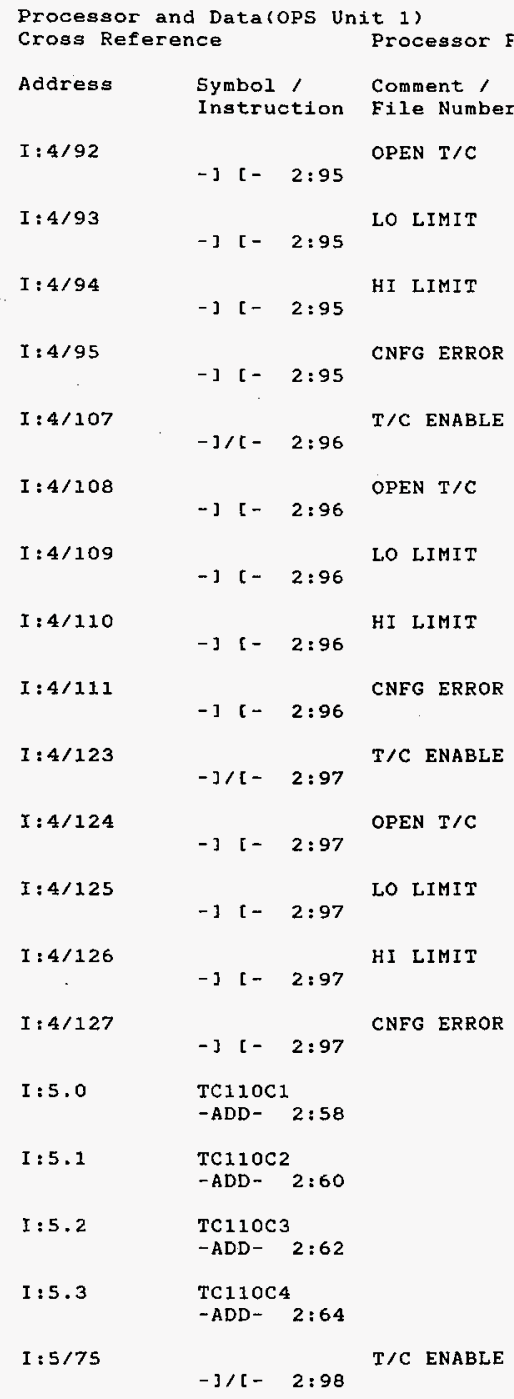




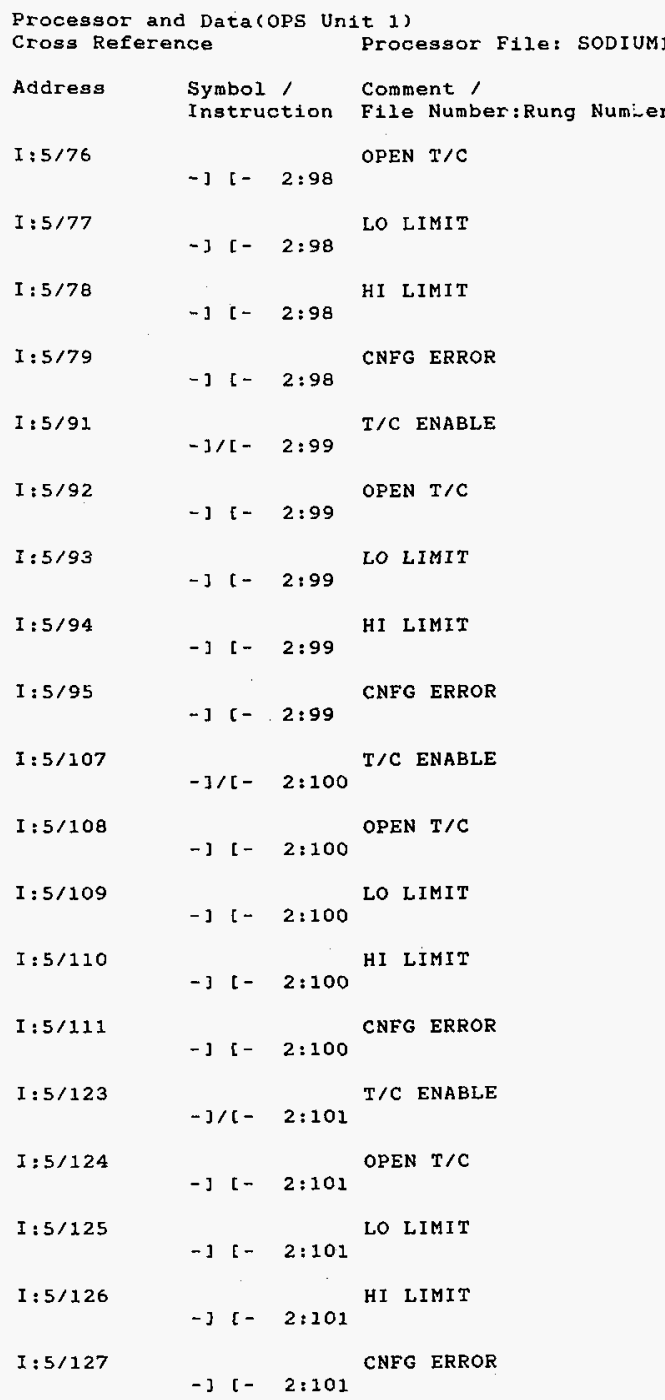

October 23, 1996 Page 10

HNF-SD-FF-CSWD-60Rev. 0

\section{Page 10}




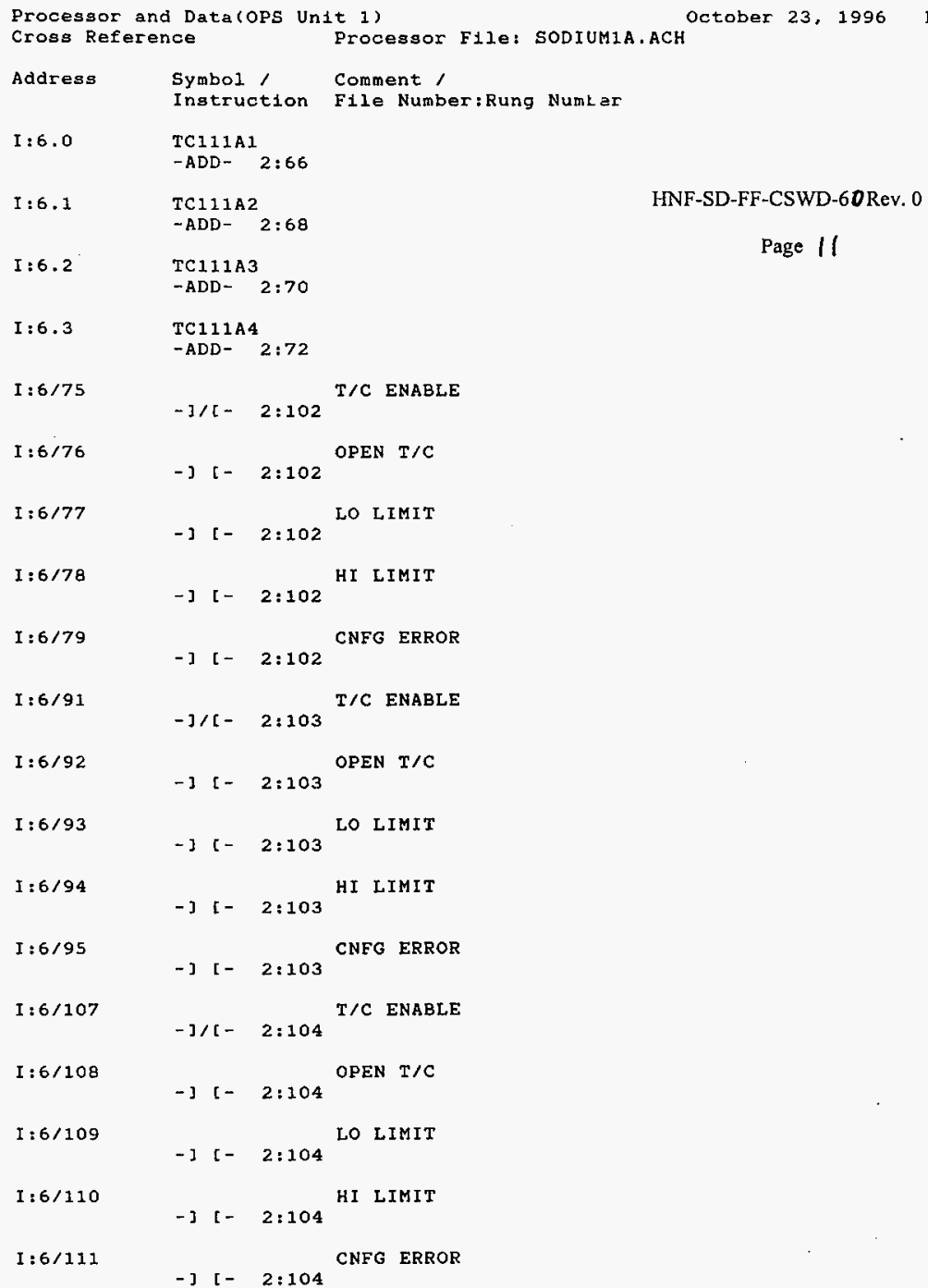

HNF-SD-FF-CSWD-60Rev. 0

Page II 


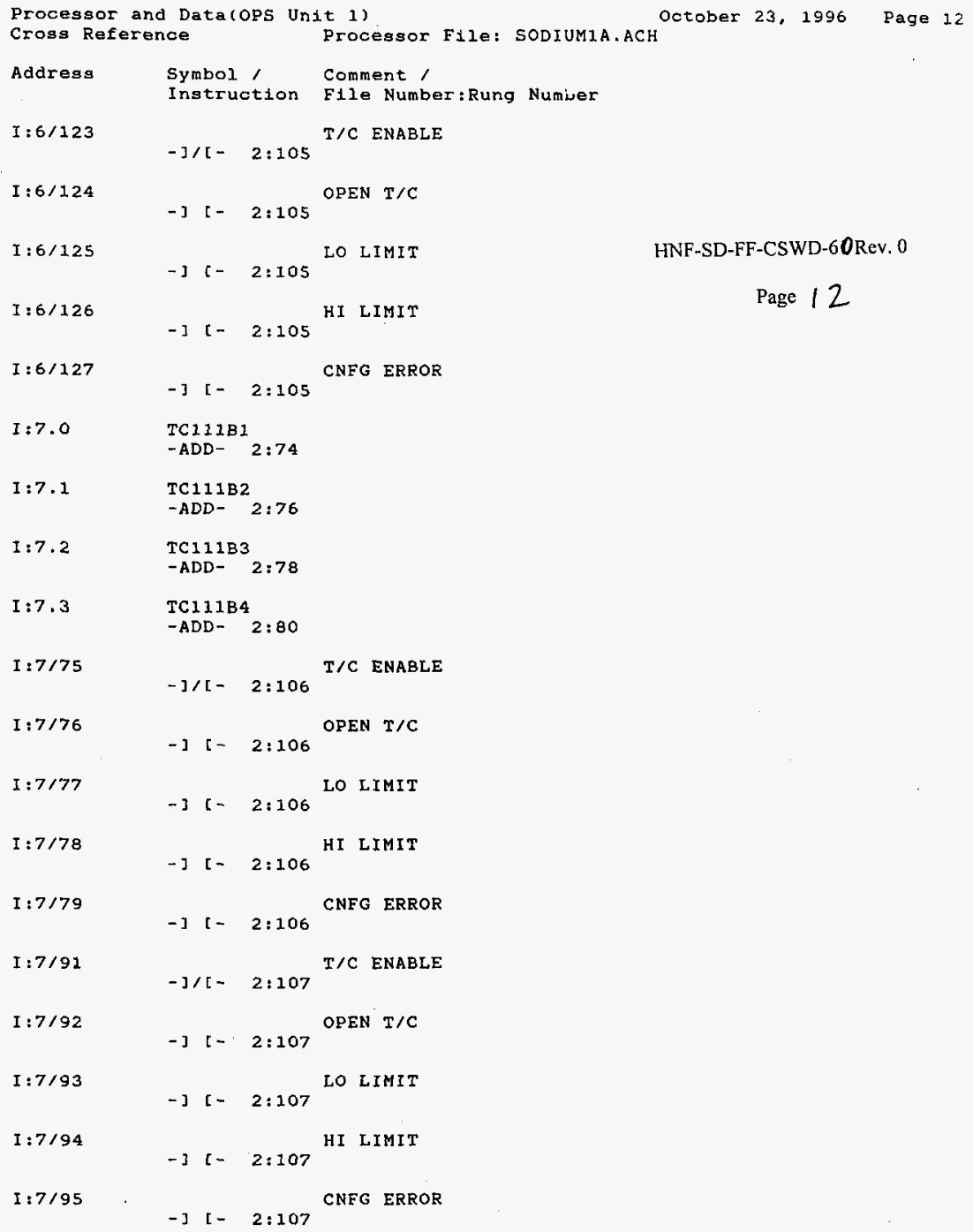

HNF-SD-FF-CSWD-60Rev. 0

\section{Page 12}




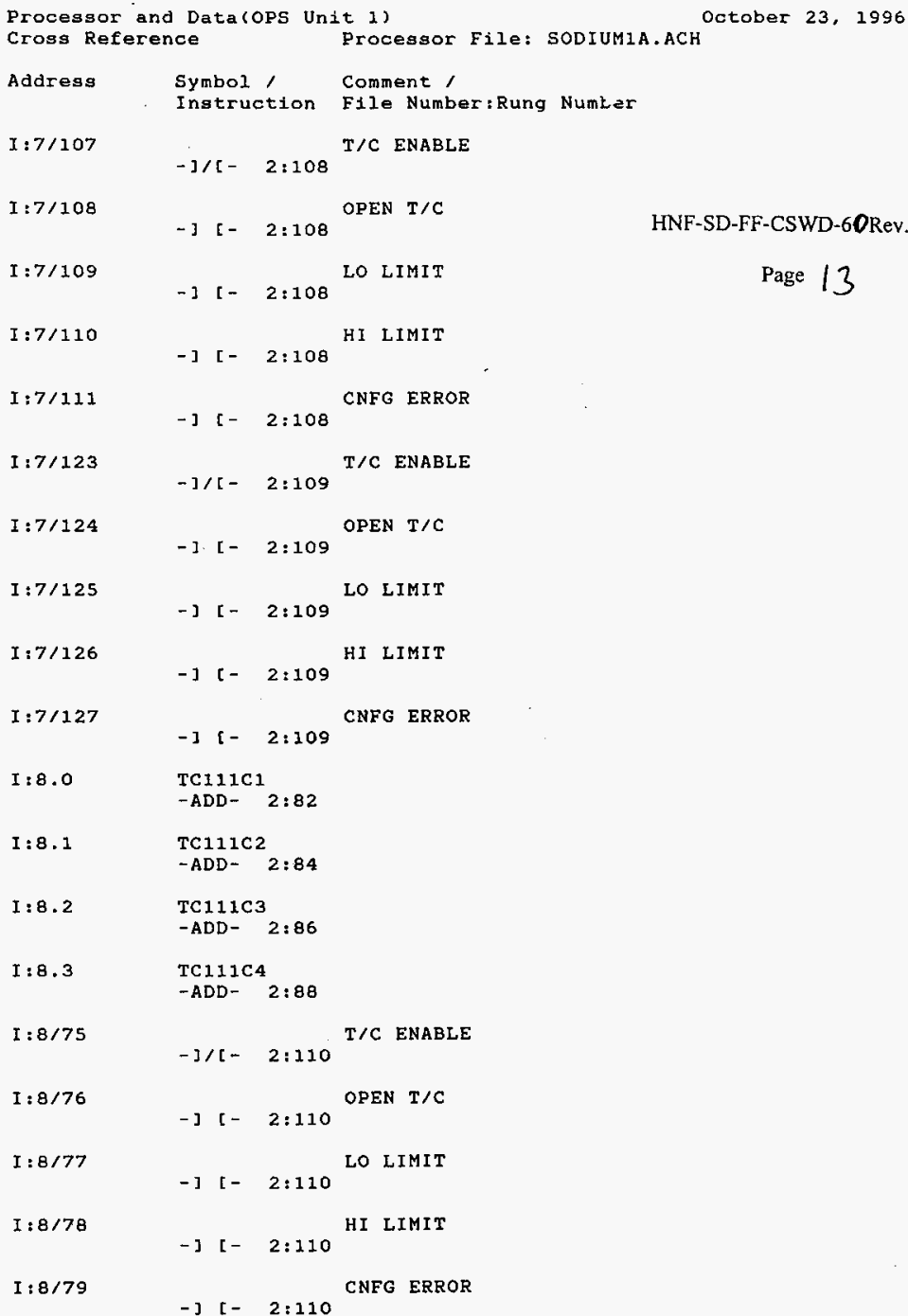




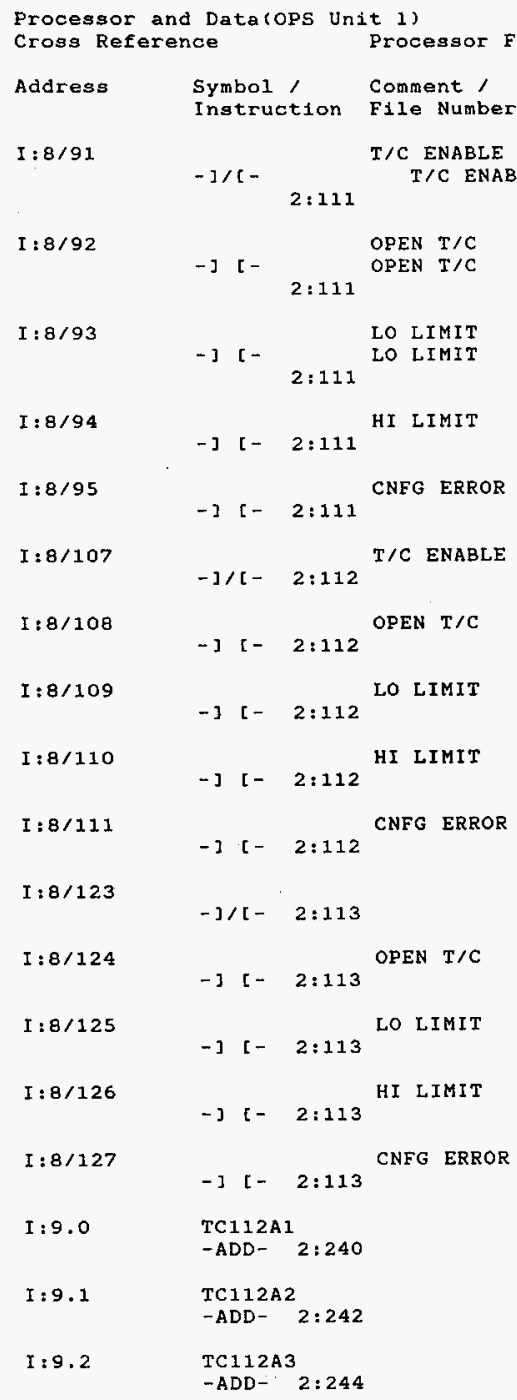

October 23, 1996 Page 14

HNF-SD-FF-CSWD-60Rev. 0

Page / 4 


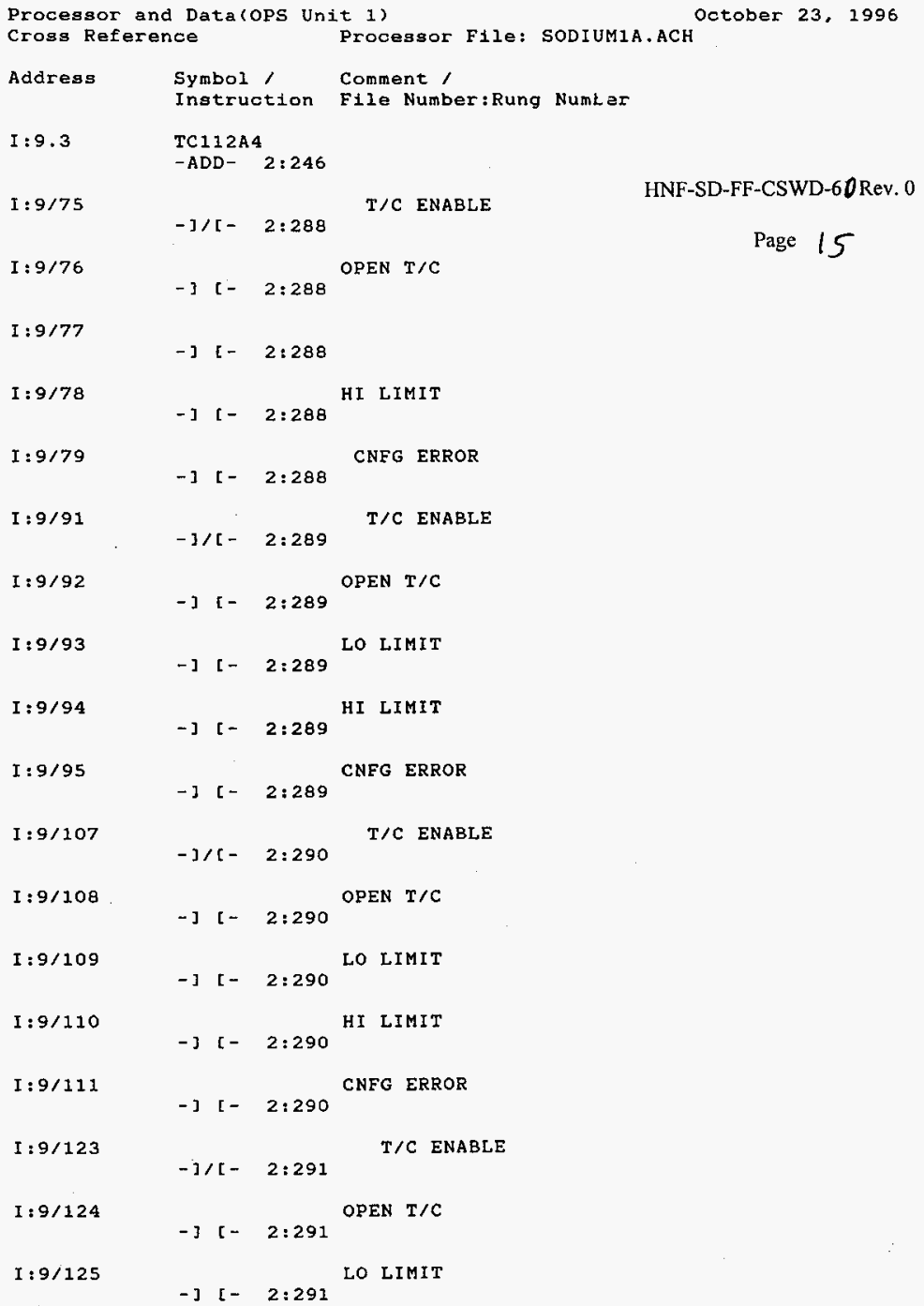




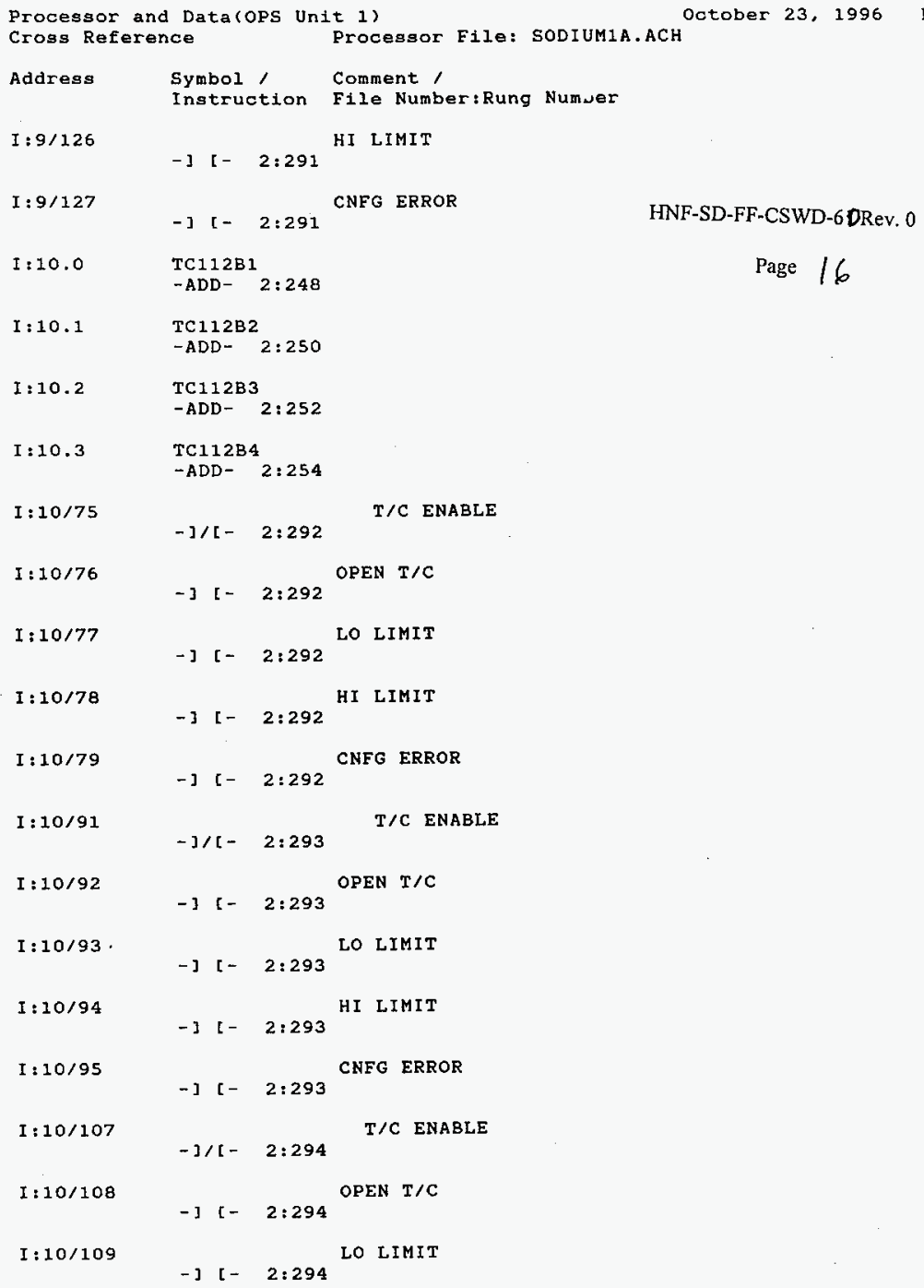




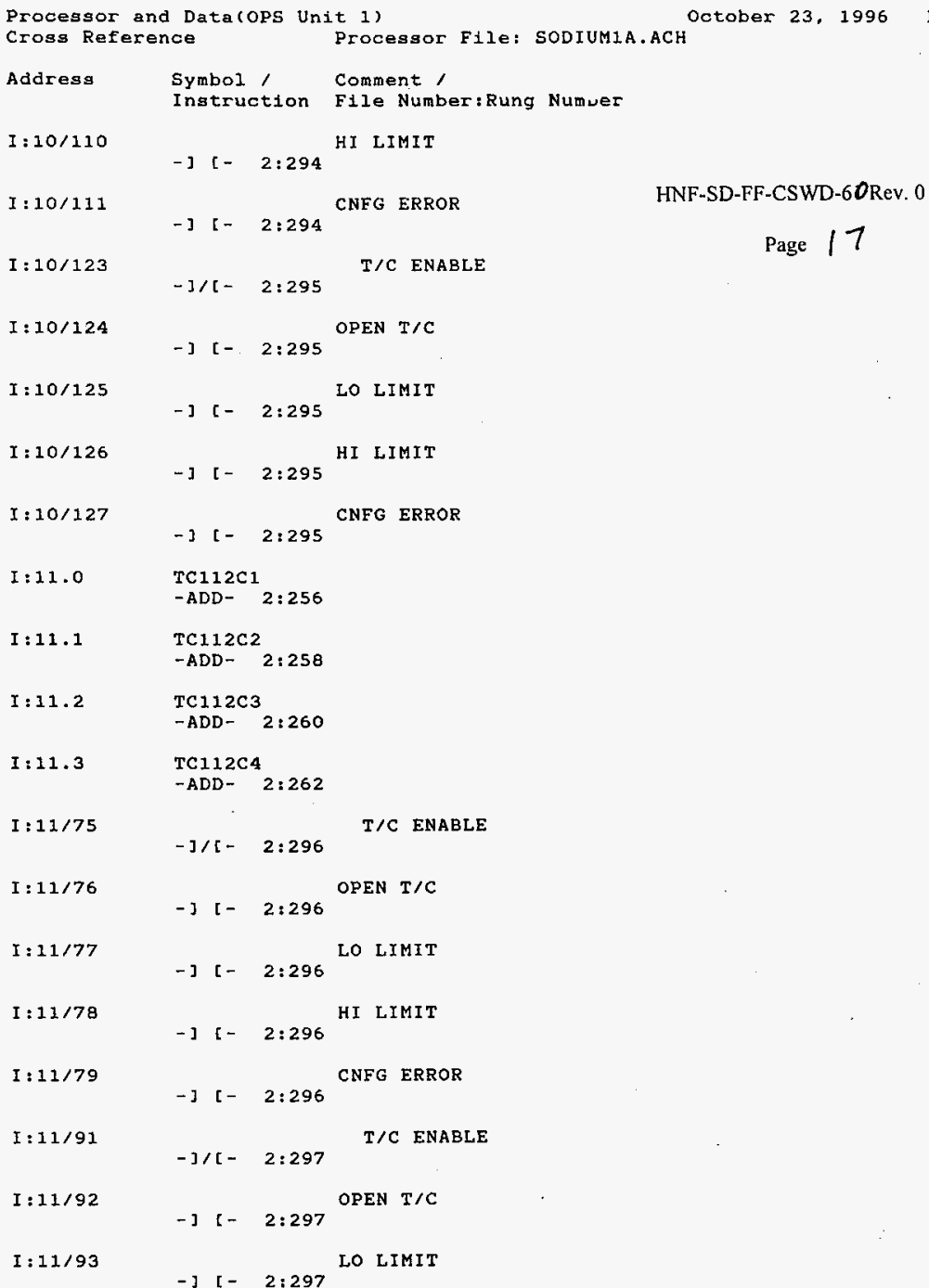




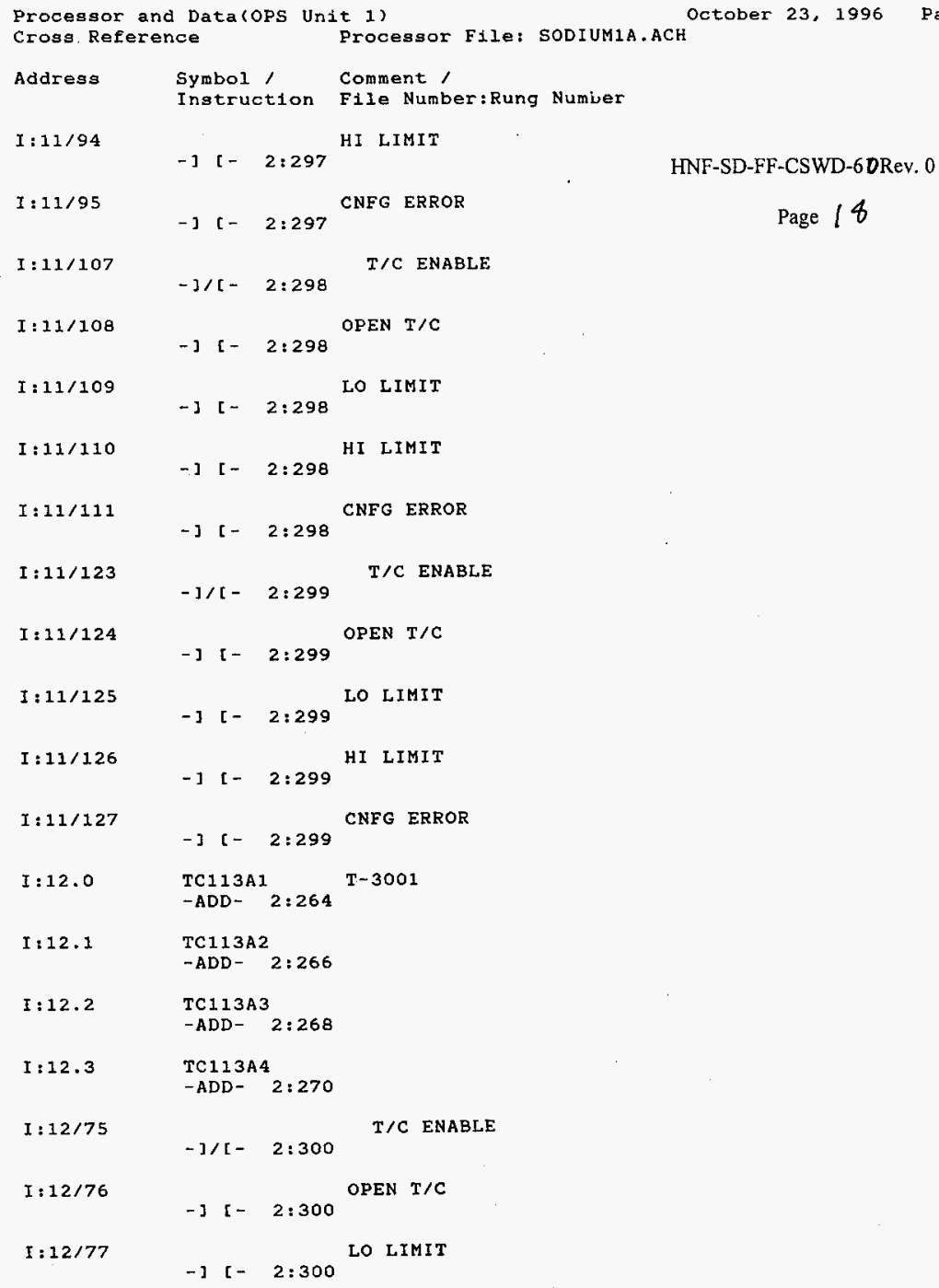

HNF-SD-FF-CSWD-6DRev. 0

Page 18 


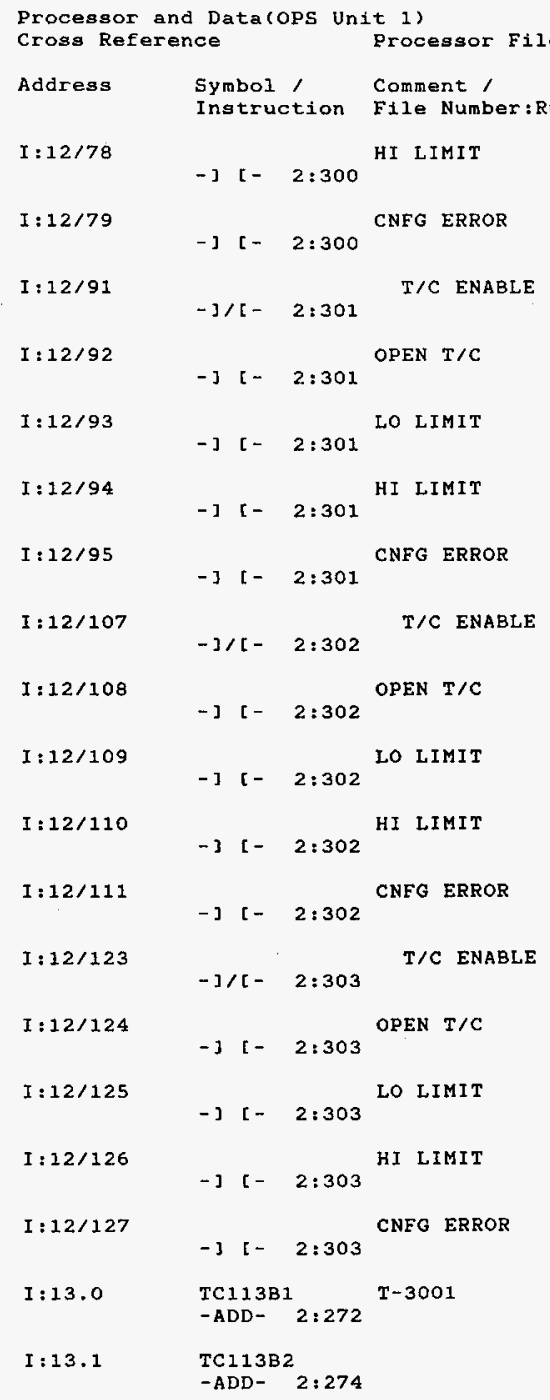




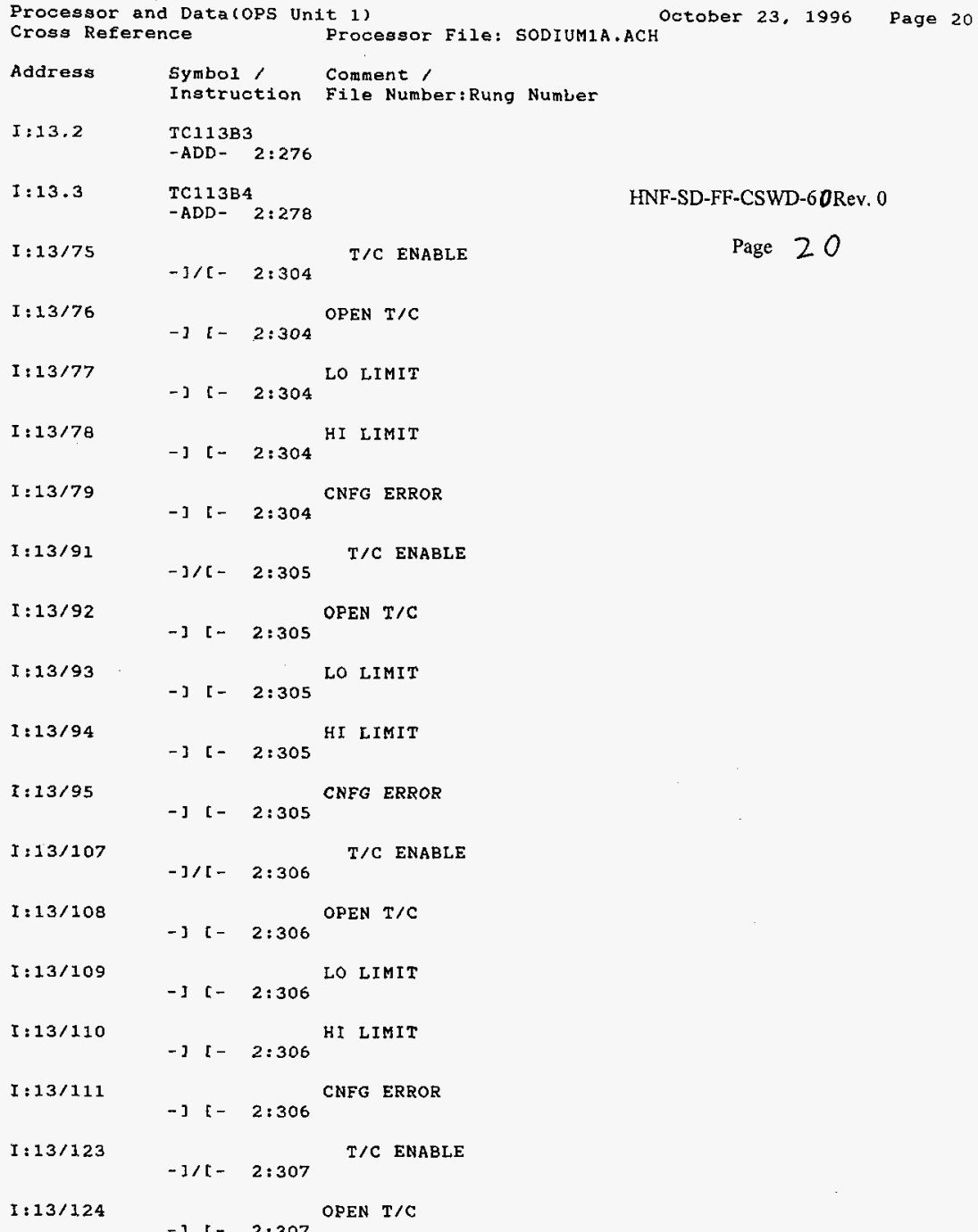




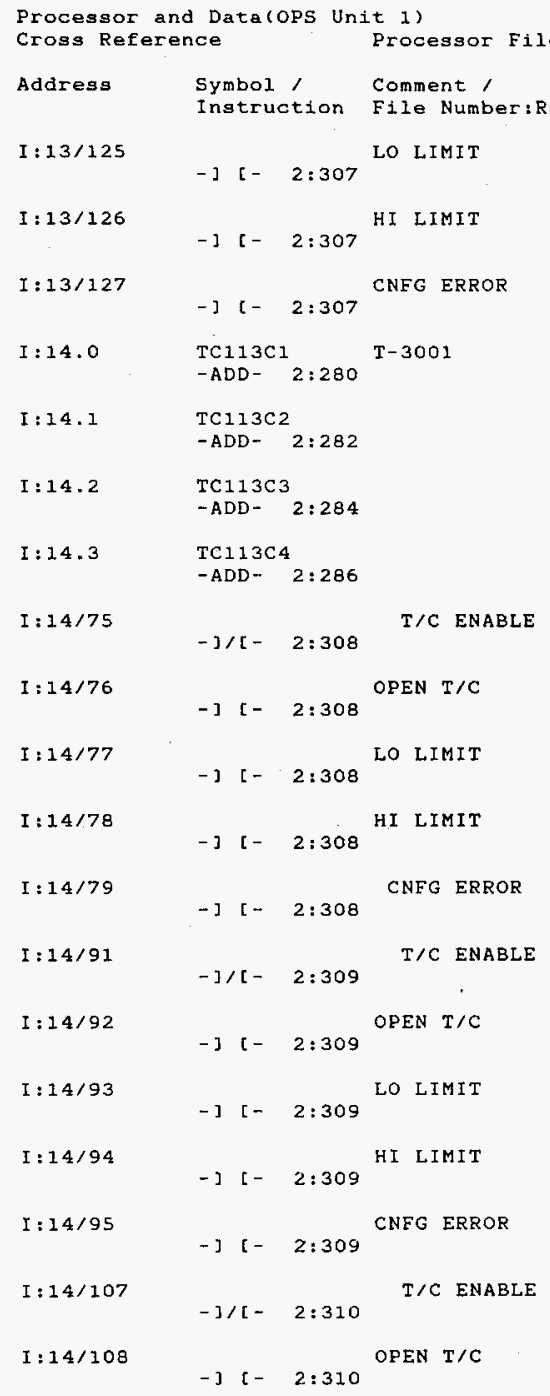


THIS PA T INTENTIONALLY

L. HT BLANK 


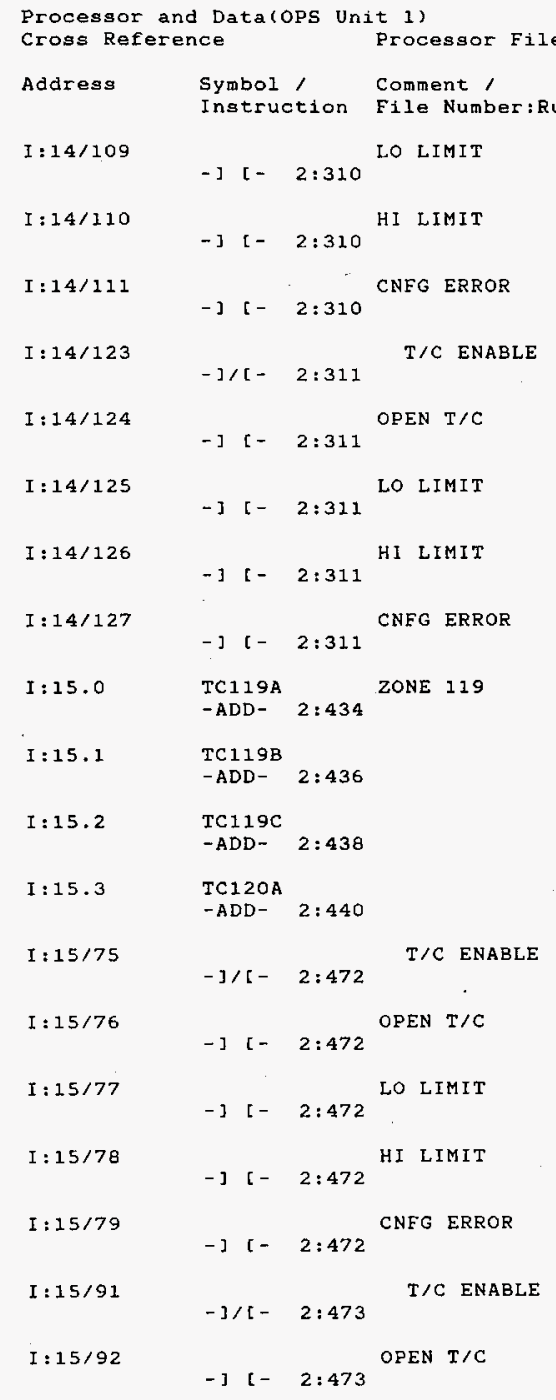


THIS PAGE INTENTIONALLY
LEFT BLANK 


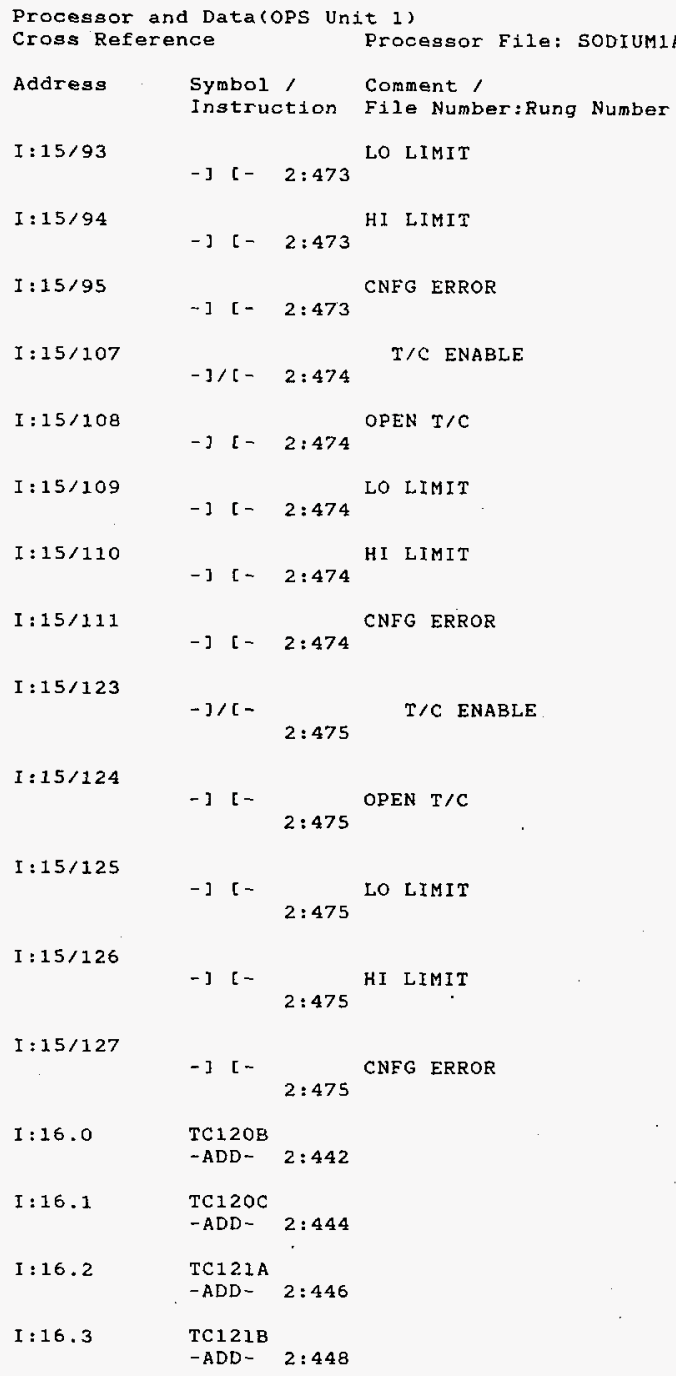

HNF-SD-FF-CSWD-60 Rev. 0

Page 23 
THIS PACE INTENTIONALLY LEFT BLANK 


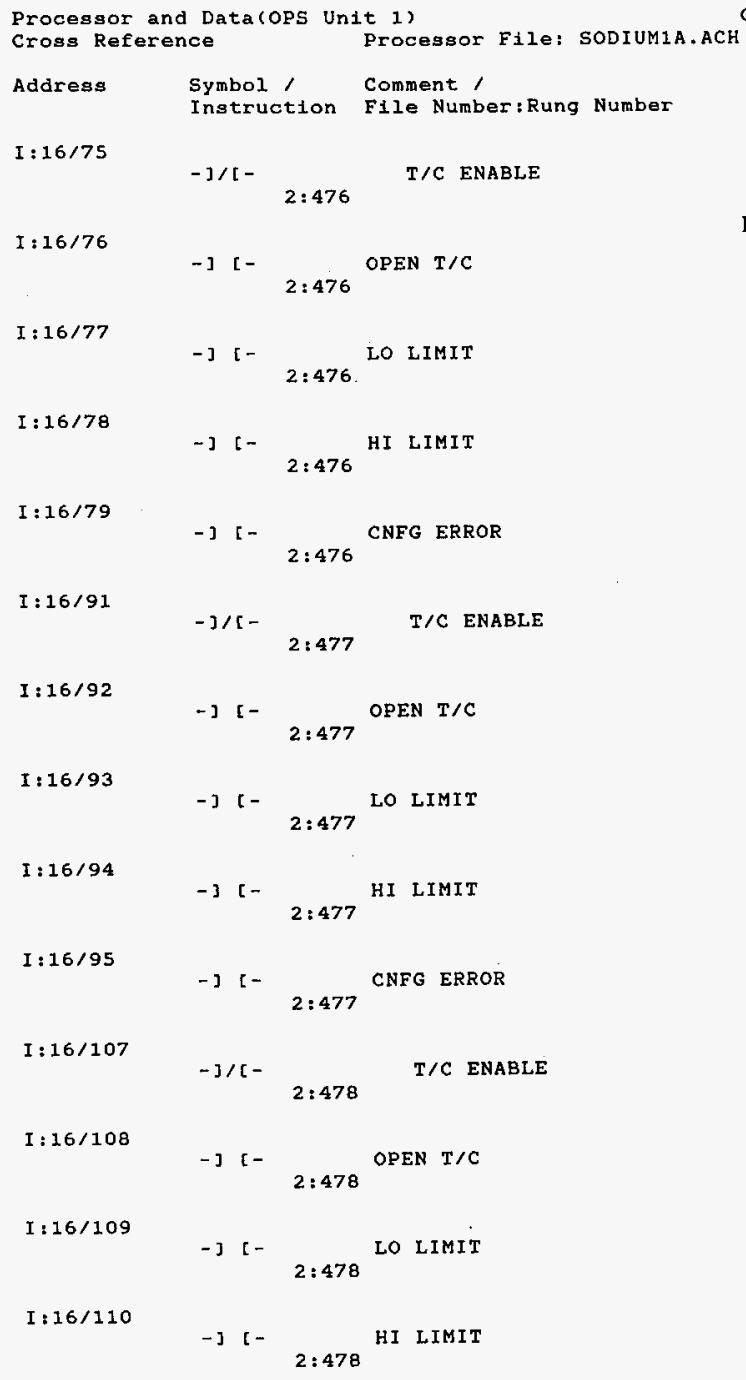


Processor and Data(OPS Unit 1)

October 23, 1996 Page 2:

Cross Reference

Adiress

$I: 16 / 111$

- ] $[-\quad 2: 478$ CNFG ERROR

I : 16/123

$$
-3 /[-\quad 2: 479 \quad T / C \text { ENABLE }
$$

$I: 16 / 124$

$$
-][-\quad 2: 479 \text { OPEN T/C }
$$

I : $16 / 125$

$$
-][-2: 479 \text { LO LIMIT }
$$

I : $16 / 126$

$$
\text { - ] [ } 2: 479 \text { HI LIMIT }
$$

I: $16 / 127$

$$
\text { - ] [ - } 2: 479 \text { CNEG ERROR }
$$

$I: 17.0$

TC121C

-ADD- $2: 450$

I $: 17.1$

TC122A

-ADD- 2:452

I $: 17.2$

$$
-] /[-
$$

T/C ENABLE

$I: 17 / 76$

$$
-3[-\quad 2: 480 \text { OPEN T/C }
$$

I : $17 / 77$

$$
-][-\quad 2: 480 \text { LO LIMIT }
$$

I $: 17 / 78$

$$
-] \text { I - } 2: 480 \text { HI LIMIT }
$$

I:17/79

$$
\text { - ] [ } 2: 480^{\text {CNFG ERROR }}
$$

HNF-SD-FF-CSWD-60Rev. 0

\section{Page 25}




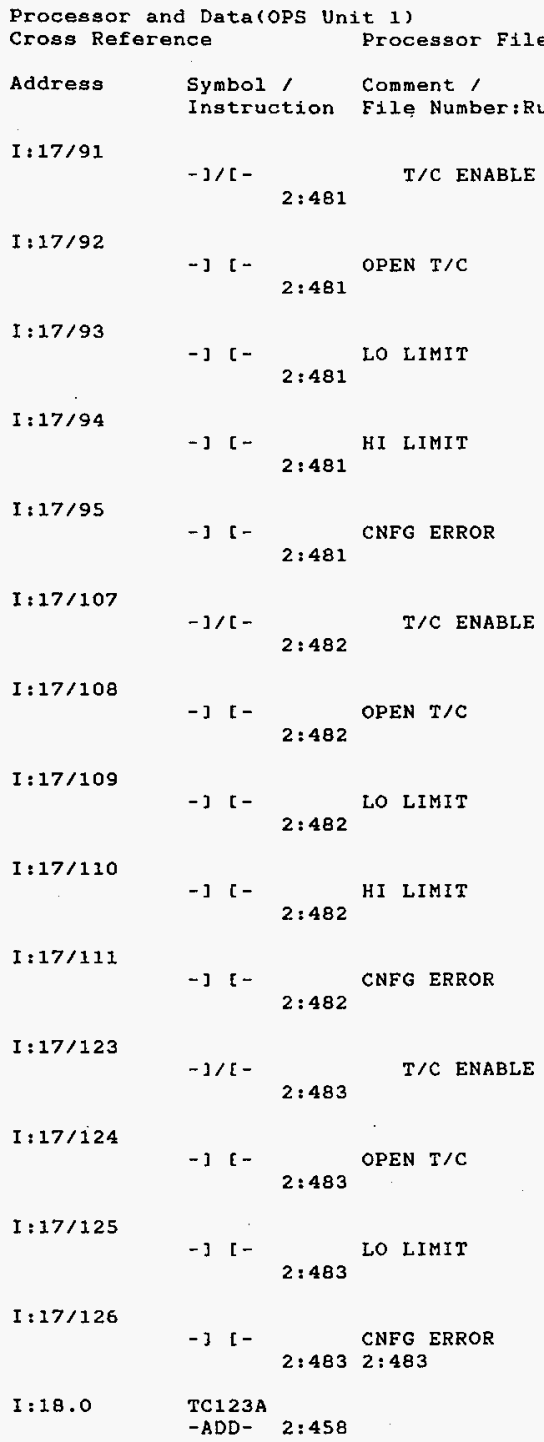

HNF-SD-FF-CSWD- 60 Rev, 0

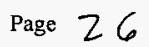




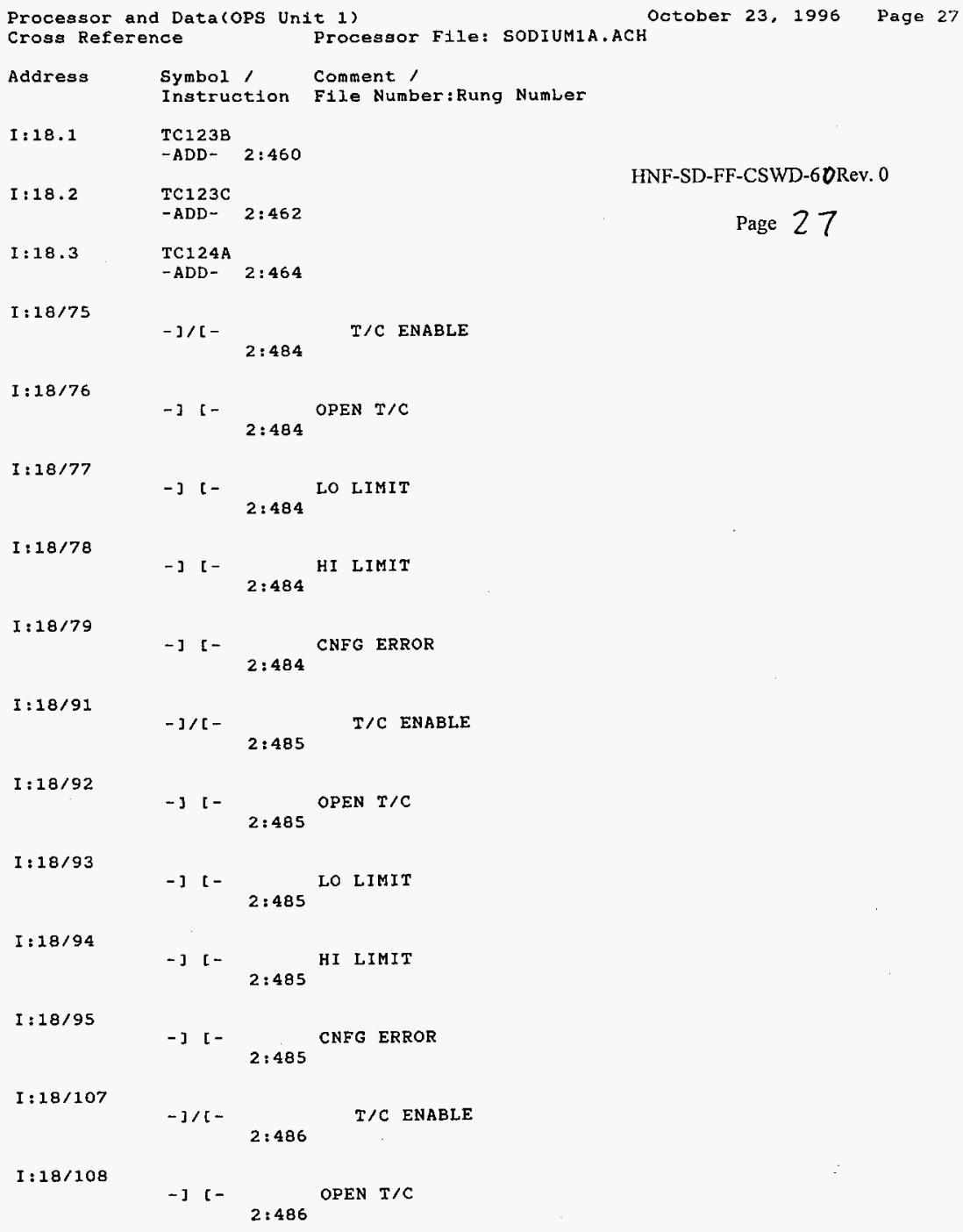




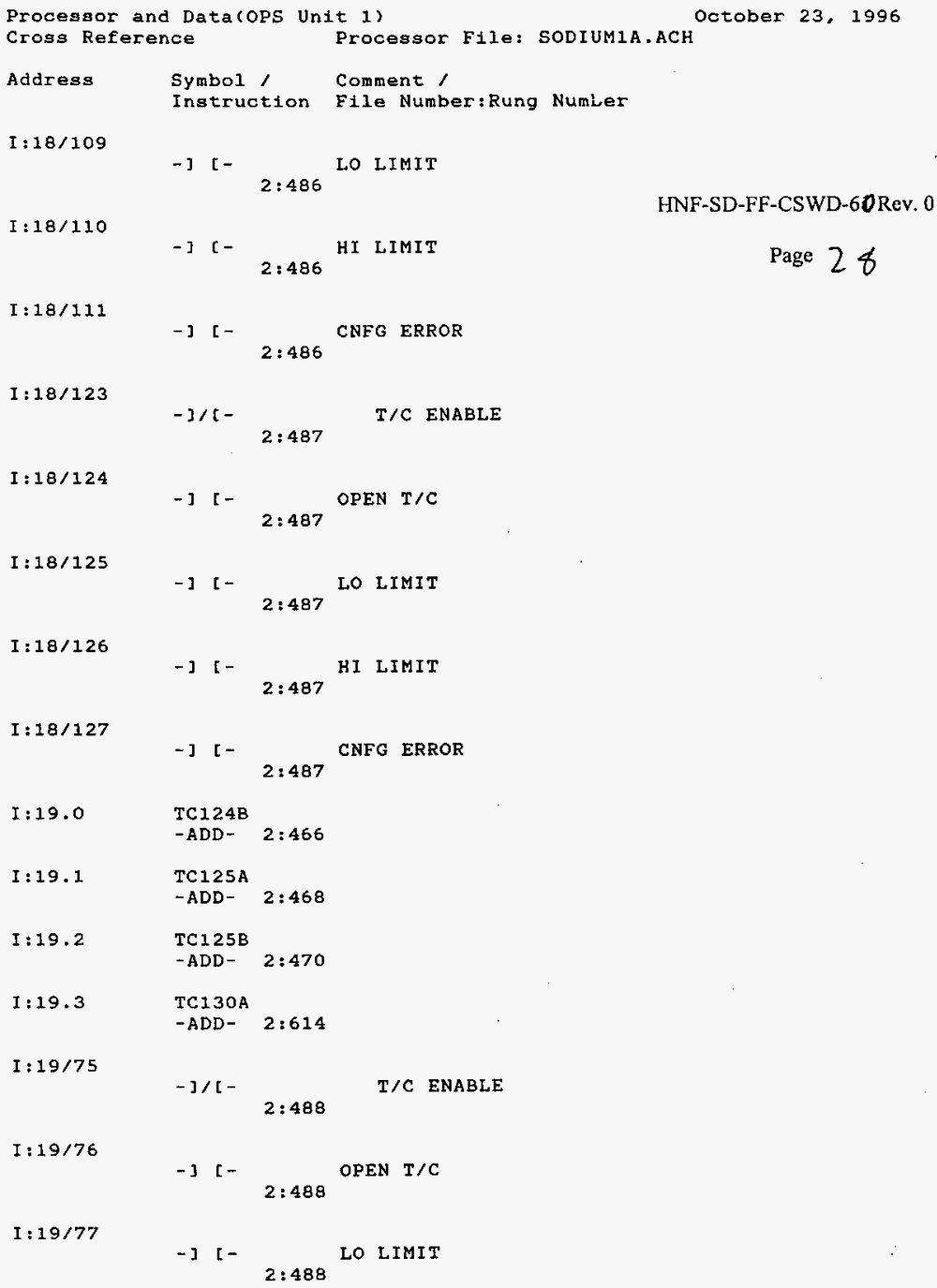




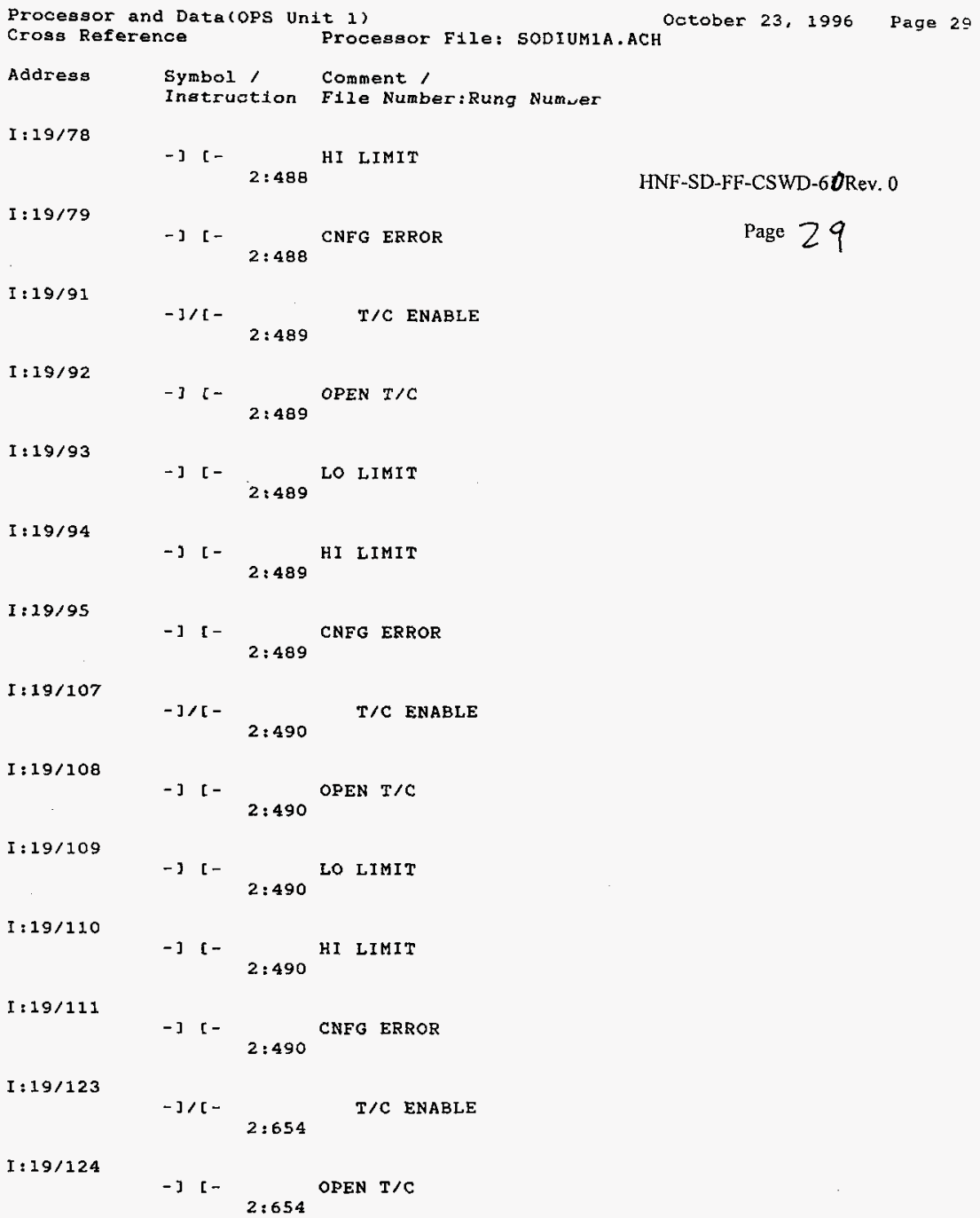

HNF-SD-FF-CSWD- 60 Rev. 0

Page 29 


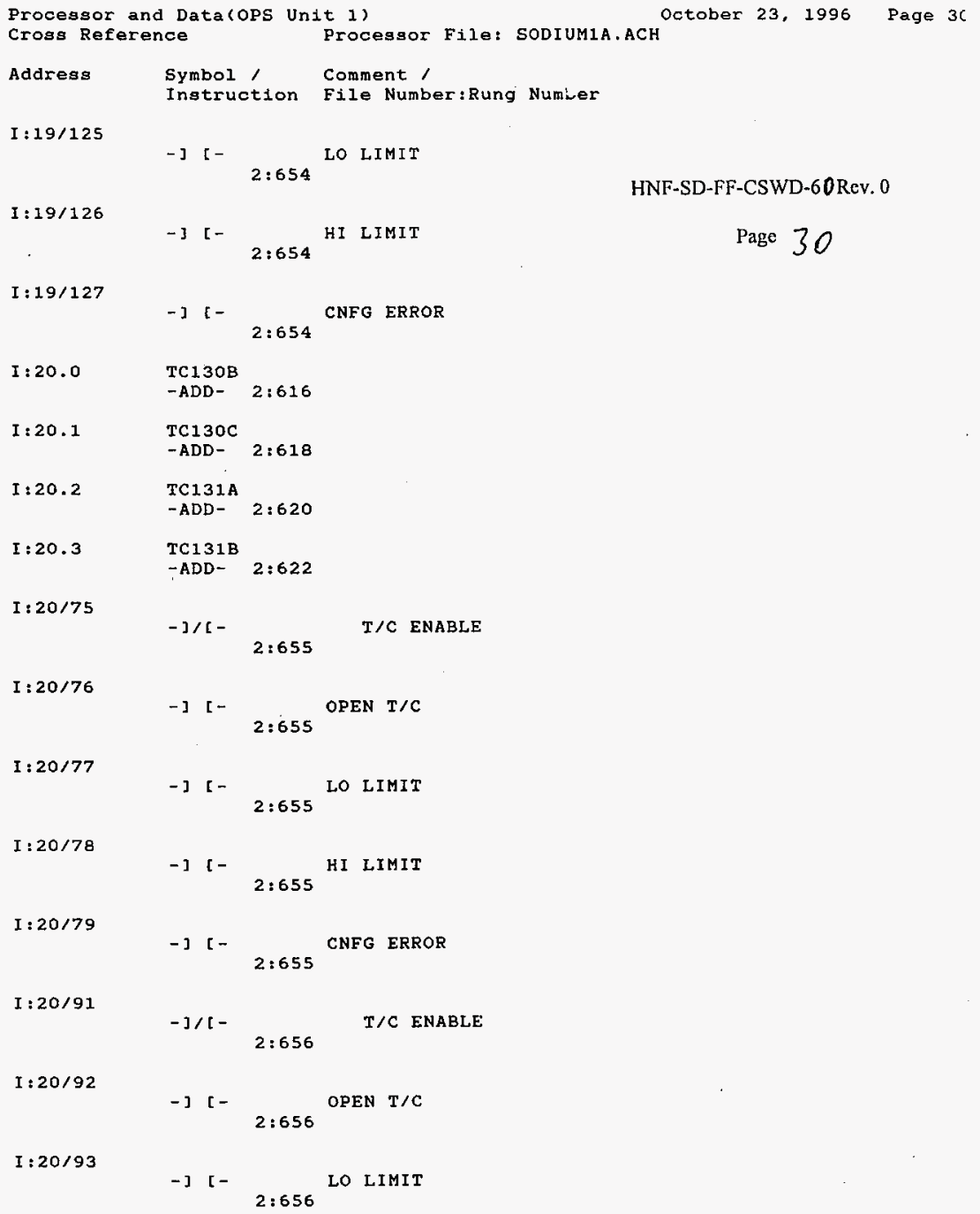




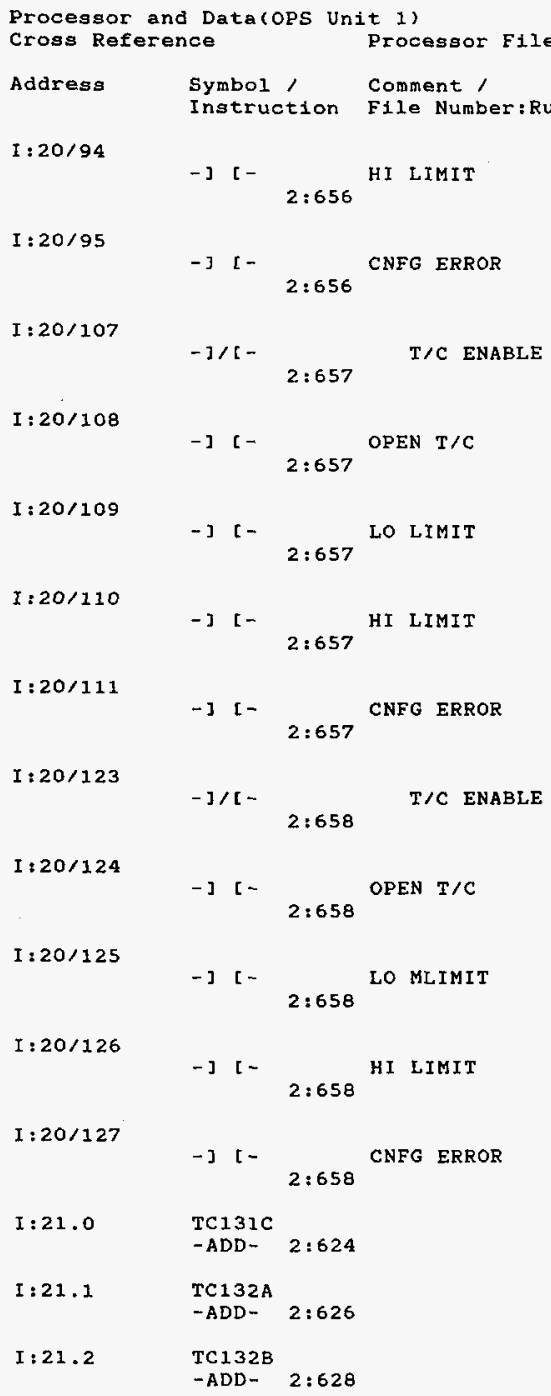




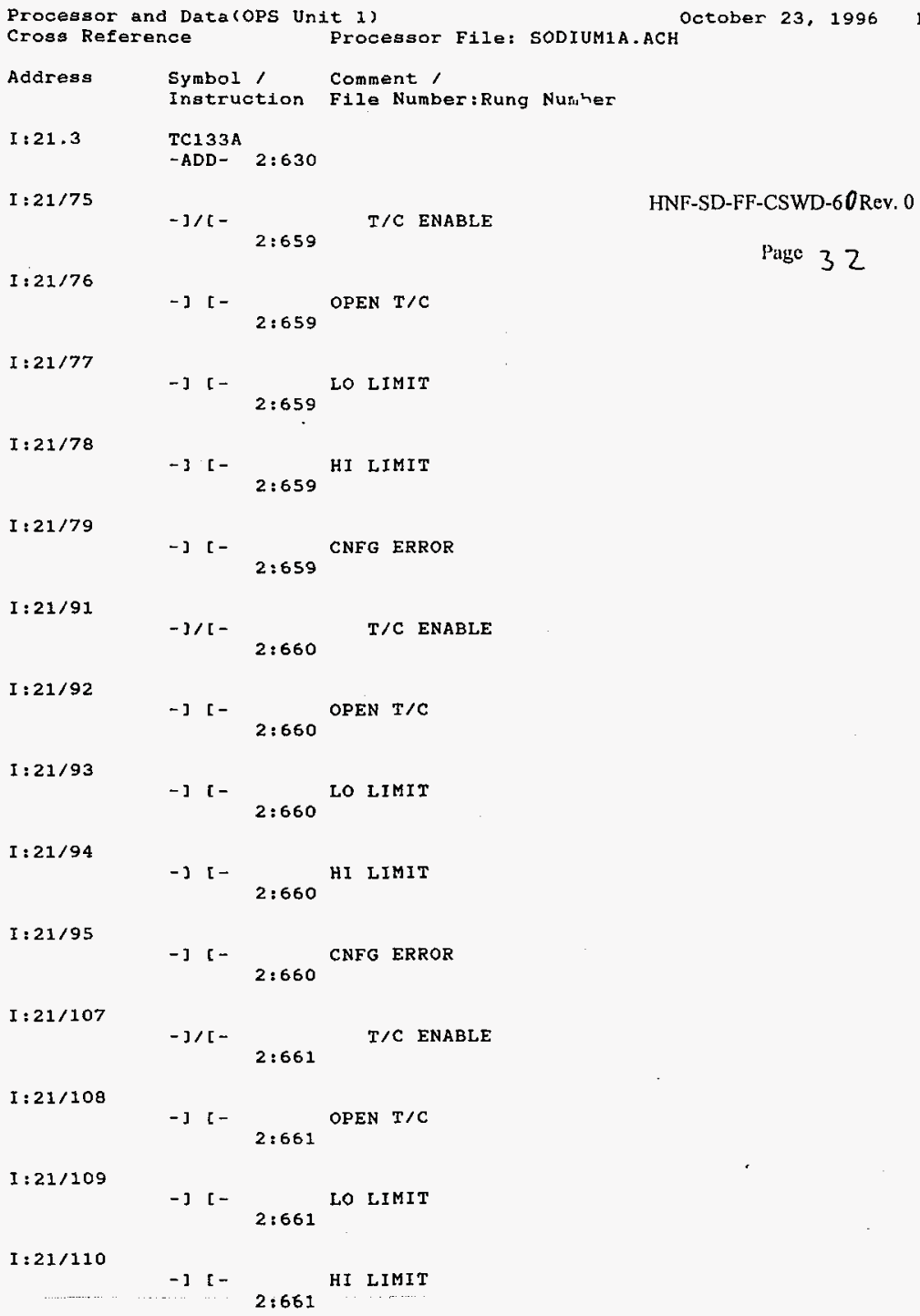

Address

Symbol,

Comment,

Instruction

File Number:Rung Number

I $: 21 \cdot 3$

TC133A

-ADD- $2: 630$

$I: 21 / 75$

$-] /[-\quad 2: 659 \quad$ T/C ENABLE

HNF-SD-FF-CSWD-60Rev. 0

$I: 21 / 76$

$-][-\quad 2: 659$ OPEN $T / C$

$I: 21 / 77$

$-][-\quad 2: 659$ LO LIMIT

$I: 21 / 78$

$-3\left[-\quad 2: 659^{H I}\right.$ LIMIT

I $: 21 / 79$

- $]$ [- $2: 659$ CNFG ERROR

I : 21/91

$-] /[-\quad 2: 660 \quad$ T/C ENABLE

$I: 21 / 92$

$-][-\quad 2: 660$ OPEN T/C

$I: 21 / 93$

- ] [- $2: 660^{\text {LO LIMIT }}$

$I: 21 / 94$

$-3\left[-\quad 2: 660^{\text {HI LIMIT }}\right.$

$I: 21 / 95$

- ] [- 2:660 CNFG ERROR

I $: 21 / 107$

$$
-] /[-\quad 2: 661 \quad \text { T/C ENABLE }
$$

I: $21 / 108$

$$
-][-\quad 2: 661 \text { OPEN T/C }
$$

I : 21/109

$$
\text { - ] [- 2:661 LO LIMIT }
$$

$I: 21 / 110$

$$
-1[-\quad 2: 66 I \text { HI LIMIT }
$$




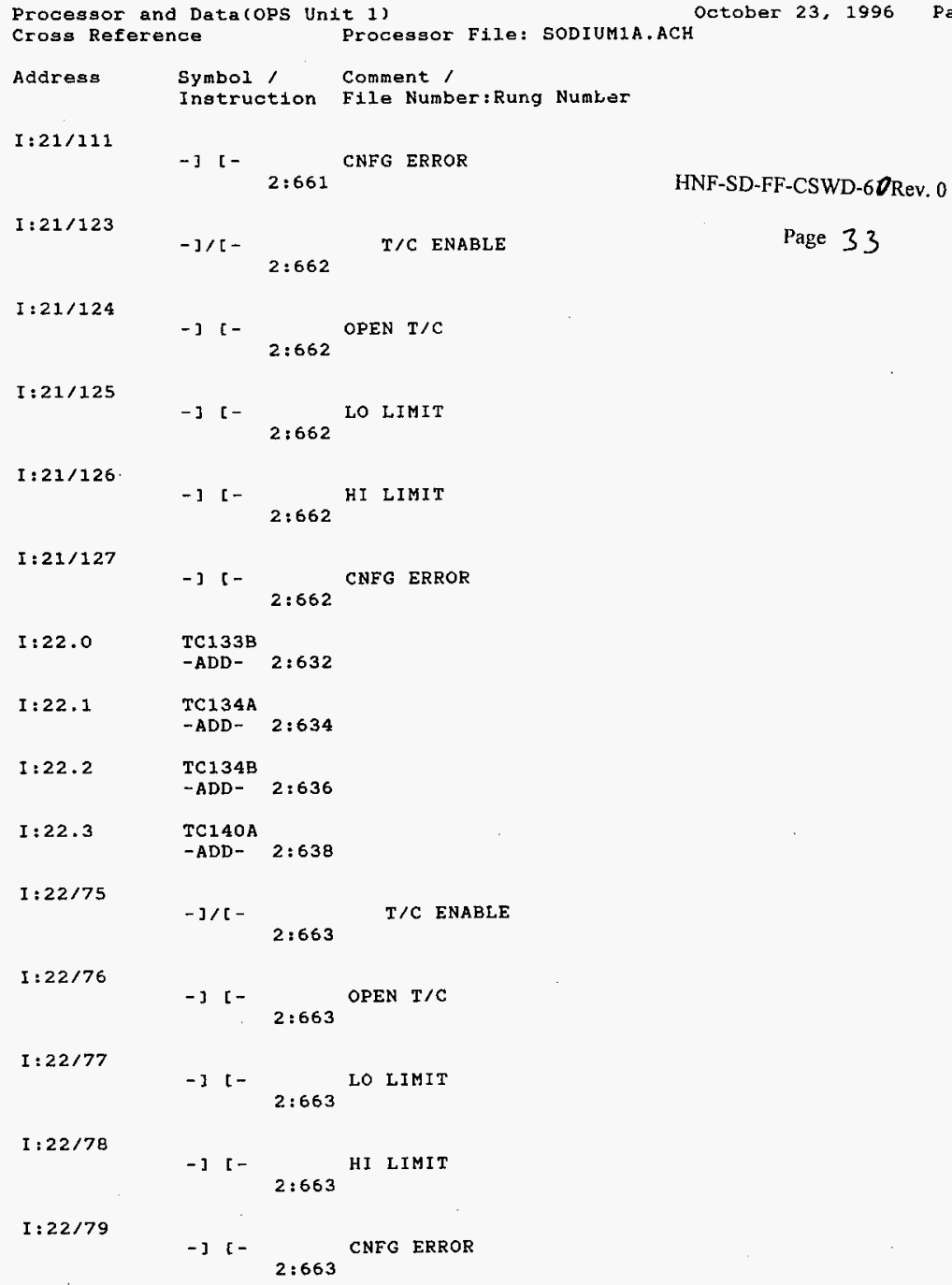




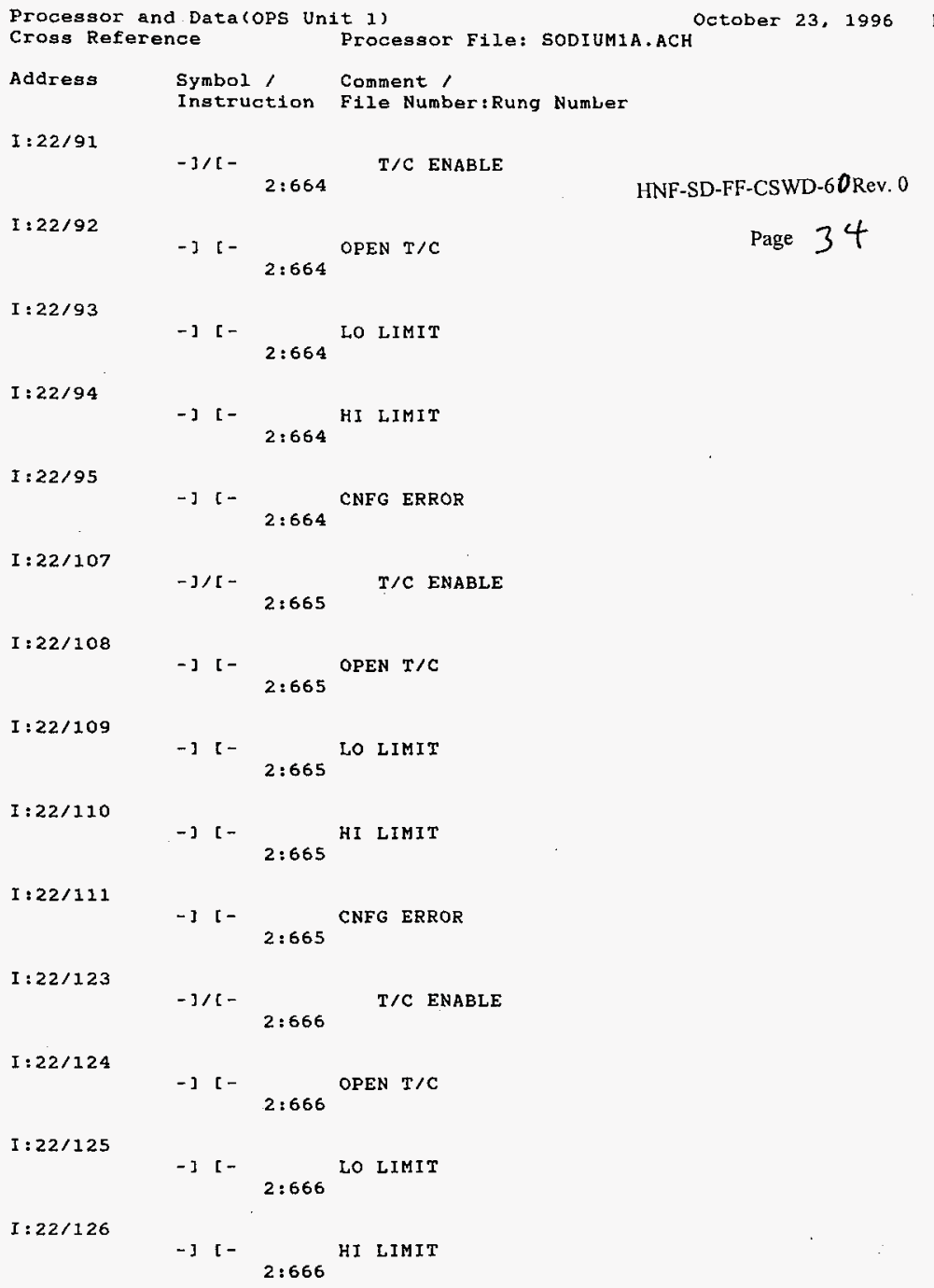




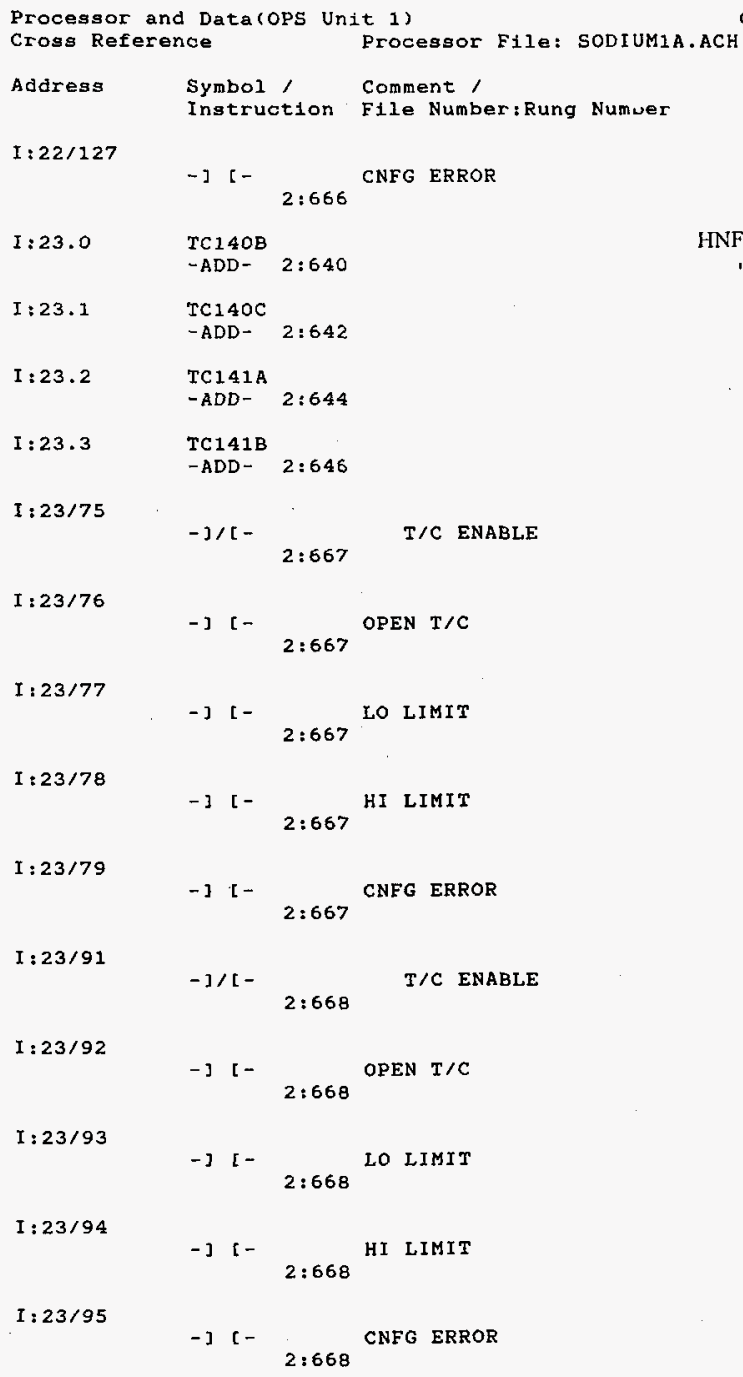




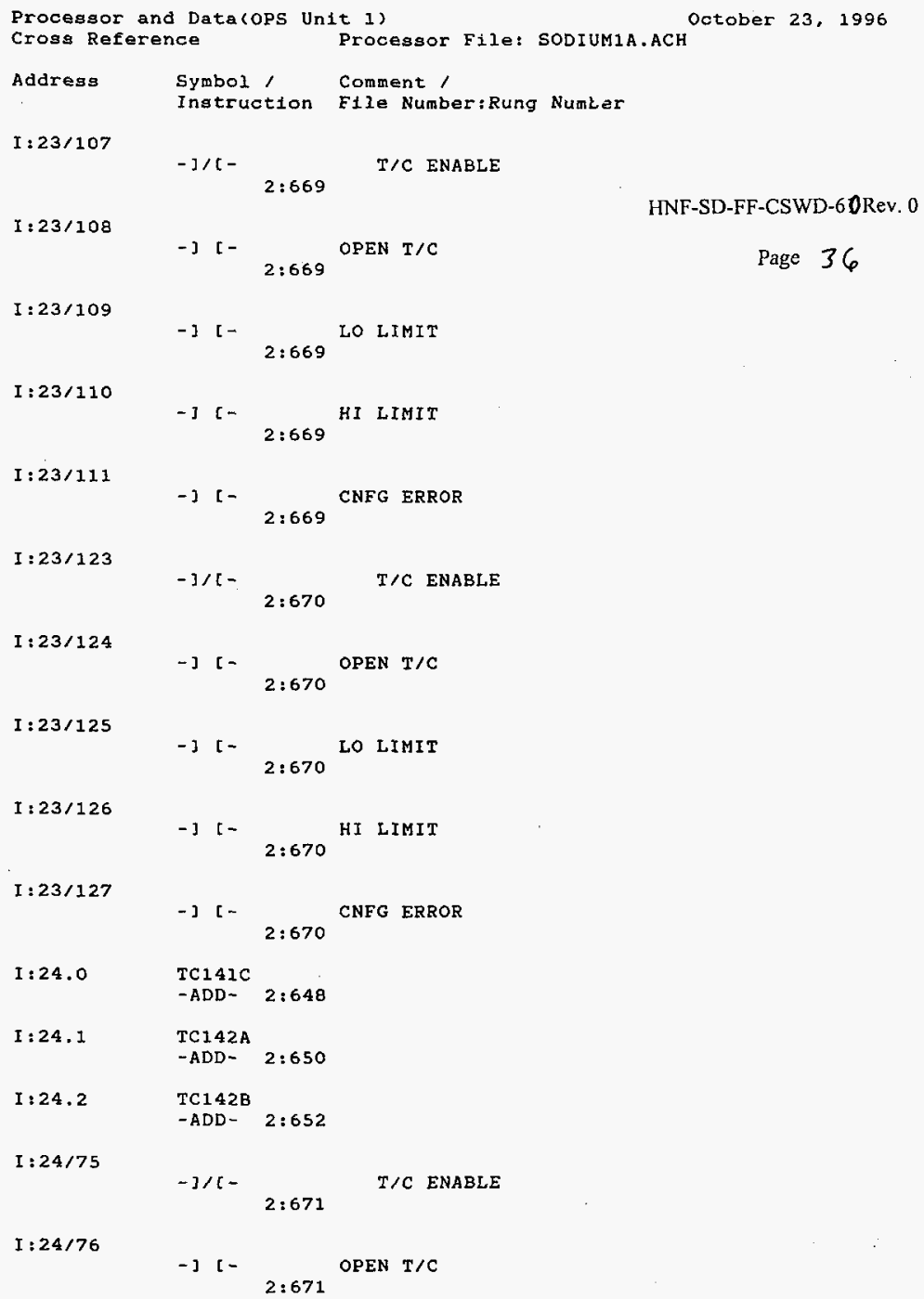

$$
\text { - ] [ - } 2: 671 \text { OPEN T/C }
$$




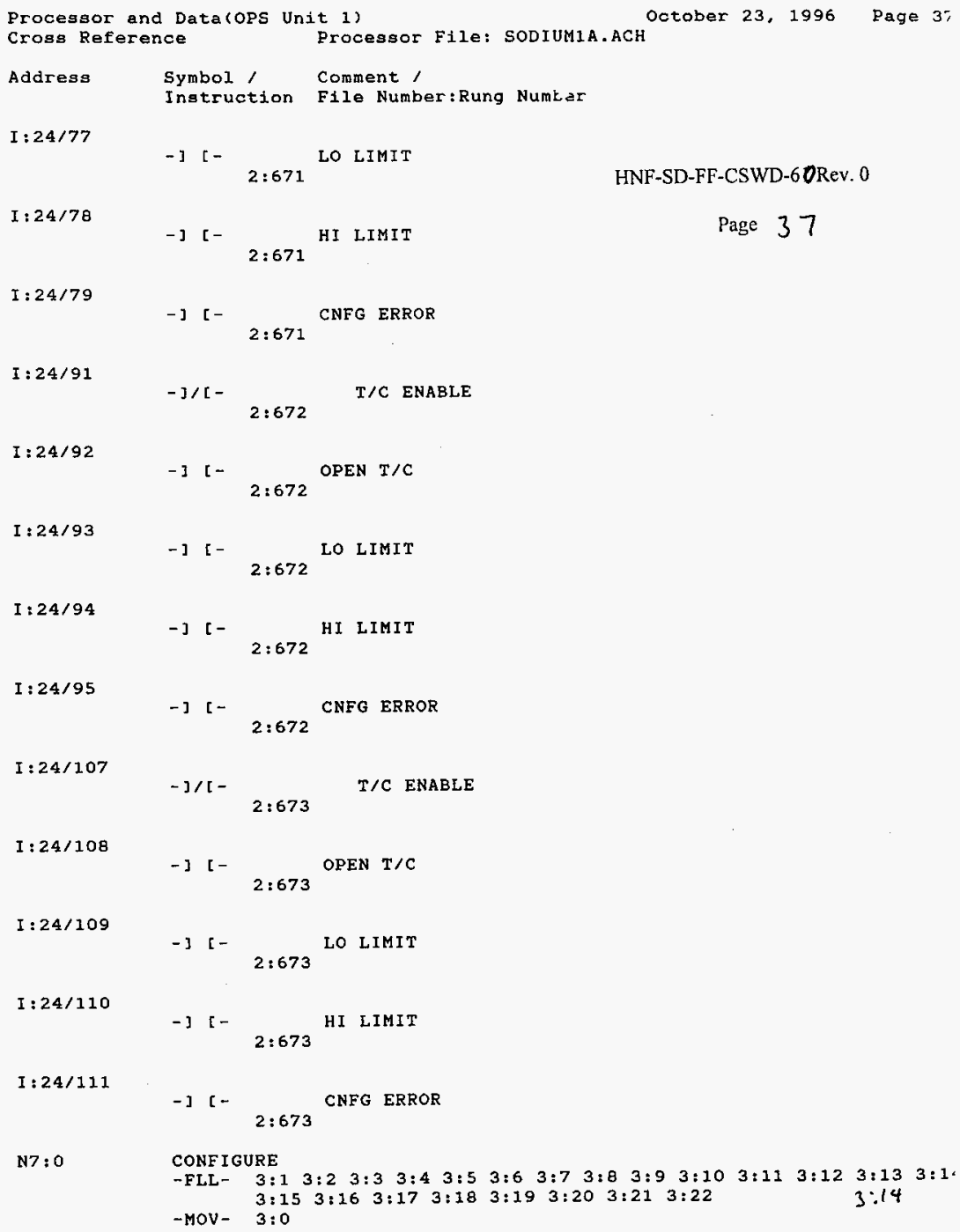

HNF-SD-FF-CSWD-60Rev. 0

\section{Page 37}


Tag Name Tag Type Description PLCDevice PLCl/O Ladder Logic Rungs

\begin{tabular}{|c|c|c|c|c|c|c|}
\hline C110A1 & $A A$ & T-3002 Zone A1 & DEV1:N7:1 & 1D0 & ADD & $2: 422: 153$ \\
\hline & & & & & GRT & $2: 432: 121$ \\
\hline & & & & & LES & $2: 121$ \\
\hline & & & & & MOV & $2: 43$ \\
\hline C110A2 & AA & T-3002 Zone A2 & DEV1:N7:2 & 1D3 & ADD & $2: 442: 154$ \\
\hline & & & & & GRT & $2: 452: 122$ \\
\hline & & & & & LES & $2: 122$ \\
\hline & & & & & MOV & $2: 45$ \\
\hline C110A3 & $A A$ & T-3002 Zone A3 & DEV1:N7:3 & $1 \mathrm{D} 6$ & ADD & $2: 462: 155$ \\
\hline & & & & & GRT & $2: 472: 123$ \\
\hline & & & & & LES & $2: 123$ \\
\hline & & & & & MOV & $2: 47$ \\
\hline C110A4 & $A A$ & T-3002 Zone A4 & DEV1:N7:4 & 1D9 & $\mathrm{ADD}$ & $2: 482: 156$ \\
\hline & & & & & GRT & $2: 492: 124$ \\
\hline & & & & & LES & $2: 124$ \\
\hline & & & & & MOV & $2: 49$ \\
\hline C110B1 & $A A$ & T-3002 Zone B1 & DEV1:N7:5 & $1 E 0$ & ADD & $2: 502: 159$ \\
\hline & & & & & GRT & $2: 512: 126$ \\
\hline & & & & & LES & $2: 126$ \\
\hline & & & & & MOV & $2: 51$ \\
\hline $\mathrm{C} 110 \mathrm{~B} 2$ & $A A$ & T-3002 Zone B2 & DEV1:N7:6 & $1 \mathrm{E} 3$ & ADD & $2: 522: 160$ \\
\hline & & & & & GRT & $2: 532: 127$ \\
\hline & & & & & LES & $2: 127$ \\
\hline & & & & & MOV & $2: 53$ \\
\hline $\mathrm{C} 110 \mathrm{~B} 3$ & $A A$ & T-3002 Zone B3 & DEV1:N7:7 & 1E6 & ADD & $2: 542: 161$ \\
\hline & & & & & GRT & $2: 552: 128$ \\
\hline & & & & & LES & $2: 128$ \\
\hline & & & & & MOV & $2: 55$ \\
\hline $\mathrm{C} 110 \mathrm{~B} 4$ & $A A$ & T-3002 Zone B4 & DEV1:N7:8 & $1 \mathrm{E} 9$ & ADD & $2: 562: 162$ \\
\hline & & & & & GRT & $2: 572: 129$ \\
\hline & & & & & LES & $2: 129$ \\
\hline & & & & & MOV & $2: 57$ \\
\hline $\mathrm{C} 110 \mathrm{C} 1$ & $A A$ & T-3002 Zone C1 & DEV1:N7:9 & 1Fo & ADD & $2: 582: 165$ \\
\hline & & & & & GRT & $2: 592: 131$ \\
\hline & & & & & LES & $2: 131$ \\
\hline & & & & & MOV & $2: 59$ \\
\hline $\mathrm{C} 110 \mathrm{C} 2$ & AA & T-3002 Zone C2 & DEV1:N7:10 & $1 \mathrm{~F} 3$ & ADD & $2: 602: 166$ \\
\hline & & & & & GRT & $2: 612: 132$ \\
\hline & & & & & LES & $2: 132$ \\
\hline & & & & & MOV & $2: 61$ \\
\hline C110C3 & AA & T-3002 Zone C3 & DEV1:N7:11 & $1 \mathrm{~F} 6$ & ADD & $2: 62$ 2:167 \\
\hline & & & & & GRT & $2: 632: 133$ \\
\hline & & & & & LES & $2: 133$ \\
\hline & & & & & MOV & $2: 63$ \\
\hline C110C4 & $A A$ & T-3002 Zone C4 & DEV1:N7:12 & $1 \mathrm{~F} 9$ & ADD & $2: 642: 168$ \\
\hline & & & & & GRT & $2: 652: 134$ \\
\hline & & & & & LES & $2: 134$ \\
\hline & & & & & MOV & $2: 65$ \\
\hline $111 A 1$ & AA & T-3003 Zone A1 & DEV1:N7:13 & $1 \mathrm{GO}$ & ADD & $2: 662: 171$ \\
\hline
\end{tabular}




\begin{tabular}{|c|c|c|c|c|c|c|}
\hline & & & & & GRT & $2: 672: 136$ \\
\hline & & & & & LES & $2: 136$ \\
\hline & & & & & MOV & $2: 67$ \\
\hline \multirow[t]{4}{*}{$\mathrm{C} 111 \mathrm{~A} 2$} & $A A$ & T-3003 Zone A2 & DEV1:N7:14 & $1 \mathrm{G} 3$ & ADD & $2: 682: 172$ \\
\hline & & & & & GRT & $2: 692: 137$ \\
\hline & & & & & LES & $2: 137$ \\
\hline & & & & & MOV & $2: 69$ \\
\hline \multirow[t]{4}{*}{ C111A3 } & $\mathrm{AA}$ & T-3003 Zone A3 & DEV1:N7:15 & $1 \mathrm{G} 6$ & ADD & $2: 702: 173$ \\
\hline & & & & & GRT & $2: 712: 138$ \\
\hline & & & & & LES & $2: 138$ \\
\hline & & & & & MOV & $2: 71$ \\
\hline \multirow[t]{4}{*}{ C111A4 } & $A A$ & T-3003 Zone A4 & DEV1:N7:16 & $1 \mathrm{G} 9$ & ADD & $2: 722: 174$ \\
\hline & & & & & GRT & $2: 732: 139$ \\
\hline & & & & & LES & $2: 139$ \\
\hline & & & & & MOV & $2: 73$ \\
\hline \multirow[t]{4}{*}{ C111B1 } & $A A$ & T-3003 Zone B1 & DEV1:N7:17 & $1 \mathrm{HO}$ & $\mathrm{ADD}$ & $2: 742: 177$ \\
\hline & & & & & GRT & $2: 752: 141$ \\
\hline & & & & & LES & $2: 141$ \\
\hline & & & & & MOV & $2: 75$ \\
\hline \multirow[t]{4}{*}{$\mathrm{C} 111 \mathrm{B2}$} & $A A$ & T-3003 Zone B2 & DEV1:N7:18 & $1 \mathrm{H} 3$ & $A D D$ & $2: 762: 178$ \\
\hline & & & & & GRT & 2:77 2:142 \\
\hline & & & & & LES & $2: 142$ \\
\hline & & & & & MOV & $2: 77$ \\
\hline \multirow[t]{4}{*}{ C111B3 } & $A A$ & T-3003 Zone B3 & DEV1:N7:19 & $1 \mathrm{H} 6$ & ADD & 2:78 2:179 \\
\hline & & & & & GRT & $2: 792: 143$ \\
\hline & & & & & LES & $2: 143$ \\
\hline & & & & & MOV & $2: 79$ \\
\hline \multirow[t]{4}{*}{ C111B4 } & $A A$ & T-3003 Zone B4 & DEV1:N7:20 & $1 \mathrm{H} 9$ & ADD & $2: 802: 180$ \\
\hline & & & & & GRT & $2: 812: 144$ \\
\hline & & & & & LES & $2: 144$ \\
\hline & & & & & MOV & $2: 81$ \\
\hline \multirow[t]{4}{*}{ C111C1 } & $A A$ & $\mathrm{~T}-3003$ Zone $\mathrm{C} 1$ & DEV1:N7:21 & 110 & ADD & $2: 822: 183$ \\
\hline & & & & & GRT & $2: 832: 146$ \\
\hline & & & & & LES & $2: 146$ \\
\hline & & & & & MOV & $2: 83$ \\
\hline \multirow[t]{4}{*}{$\mathrm{C} 111 \mathrm{C} 2$} & AA & T-3003 Zone C2 & DEV1:N7:22 & 113 & ADD & $2: 842: 184$ \\
\hline & & 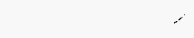 & & & GRT & $2: 852: 147$ \\
\hline & & & & & LES & $2: 147$ \\
\hline & & & & & MOV & $2: 85$ \\
\hline \multirow[t]{4}{*}{$\mathrm{C} 111 \mathrm{C} 3$} & $A A$ & T-3003 Zone C3 & DEV1:N7:23 & 116 & ADD & $2: 862: 185$ \\
\hline & & & & & GRT & $2: 872: 148$ \\
\hline & & & & & LES & $2: 148$ \\
\hline & & & & & MOV & $2: 87$ \\
\hline \multirow[t]{4}{*}{$\mathrm{C} 111 \mathrm{C} 4$} & AA & T-3003 Zone C4 & DEV1:N7:24 & 119 & ADD & $2: 882: 186$ \\
\hline & & & & & GRT & $2: 892: 149$ \\
\hline & & & & & LES & $2: 149$ \\
\hline & & & & & MOV & $2: 89$ \\
\hline \multirow[t]{2}{*}{ C112A1 } & $A A$ & T-3004 Zone A1 & DEV1:N7:25 & 1JO & ADD & $2: 2402: 347$ \\
\hline & & & & & GRT & $2: 2412: 315$ \\
\hline
\end{tabular}




\begin{tabular}{|c|c|c|c|c|c|c|}
\hline & & & & & LES & $2: 315$ \\
\hline & & & & & MOV & $2: 241$ \\
\hline \multirow[t]{4}{*}{$\mathrm{C} 112 \mathrm{~A} 2$} & $\mathrm{AA}$ & T-3004 Zone A2 & DEV1:N7:26 & $1 \mathrm{~J} 3$ & ADD & $2: 2422: 348$ \\
\hline & & & & & GRT & $2: 2432: 316$ \\
\hline & & & & & LES & $2: 316$ \\
\hline & & & & & MOV & $2: 243$ \\
\hline \multirow[t]{4}{*}{ C112A3 } & $A A$ & T-3004 Zone A3 & DEV1:N7:27 & $1 \mathrm{~J} 6$ & ADD & $2: 2442: 349$ \\
\hline & & & & & GRT & $2: 2452: 317$ \\
\hline & & & & & LES & $2: 317$ \\
\hline & & & & & MOV & $2: 245$ \\
\hline \multirow[t]{4}{*}{ C112A4 } & $\mathrm{AA}$ & T-3004 Zone A4 & DEV1:N7:28 & $1 \mathrm{~J} 9$ & ADD & $2: 2462: 350$ \\
\hline & & & & & GRT & $2: 2472: 318$ \\
\hline & & & & & LES & $2: 318$ \\
\hline & & & & & MOV & $2: 247$ \\
\hline \multirow[t]{4}{*}{ C112B1 } & $A A$ & T-3004 Zone B1 & DEV1:N7:29 & $1 \mathrm{KO}$ & ADD & $2: 2482: 353$ \\
\hline & & & & & GRT & $2: 2492: 320$ \\
\hline & & & & & LES & $2: 320$ \\
\hline & & & & & MOV & $2: 249$ \\
\hline \multirow[t]{4}{*}{ C112B2 } & $A A$ & T-3004 Zone B2 & DEV1:N7:30 & $1 \mathrm{~K} 3$ & $A D D$ & $2: 2502: 354$ \\
\hline & & & & & GRT & $2: 2512: 321$ \\
\hline & & & & & LES & $2: 321$ \\
\hline & & & & & MOV & $2: 251$ \\
\hline \multirow[t]{4}{*}{ C112B3 } & $A A$ & T-3004 Zone B3 & DEV1:N7:31 & $1 \mathrm{~K} 6$ & $\mathrm{ADD}$ & $2: 2522: 355$ \\
\hline & & & & & GRT & $2: 2532: 322$ \\
\hline & & & & & LES & $2: 322$ \\
\hline & & & & & MOV & $2: 253$ \\
\hline \multirow[t]{4}{*}{$\mathrm{C} 112 \mathrm{~B} 4$} & $A A$ & T-3004 Zone B4 & DEV1:N7:32 & $1 \mathrm{~K} 9$ & ADD & $2: 2542: 356$ \\
\hline & & & & & GRT & $2: 2552: 323$ \\
\hline & & & & & LES & $2: 323$ \\
\hline & & & & & MOV & $2: 255$ \\
\hline \multirow[t]{4}{*}{$\mathrm{C} 112 \mathrm{C} 1$} & $A A$ & T-3004 Zone C1 & DEV1:N7:33 & $1 \mathrm{LO}$ & ADD & $2: 2562: 359$ \\
\hline & & & & & GRT & $2: 2572: 325$ \\
\hline & & & & & LES & $2: 325$ \\
\hline & & & & & MOV & $2: 257$ \\
\hline \multirow[t]{4}{*}{$\mathrm{C} 112 \mathrm{C} 2$} & AA & T-3004 Zone C2 & DEV1:N7:34 & $1 \mathrm{L3}$ & ADD & $2: 2582: 360$ \\
\hline & & & & & GRT & $2: 2592: 326$ \\
\hline & & & & & LES & $2: 326$ \\
\hline & & & & & MOV & $2: 259$ \\
\hline \multirow[t]{4}{*}{$\mathrm{C} 112 \mathrm{C} 3$} & $A A$ & T-3004 Zone C3 & DEV1:N7:35 & $1 \mathrm{L6}$ & ADD & $2: 2602: 361$ \\
\hline & & & & & GRT & $2: 2612: 327$ \\
\hline & & & & & LES & $2: 327$ \\
\hline & & & & & MOV & $2: 261$ \\
\hline \multirow[t]{4}{*}{$\mathrm{C} 112 \mathrm{C} 4$} & $\mathrm{AA}$ & T-3004 Zone C4 & DEV1:N7:36 & $1 L 9$ & ADD & $2: 2622: 362$ \\
\hline & & & & & GRT & $2: 2632: 328$ \\
\hline & & & & & LES & $2: 328$ \\
\hline & & & & & MOV & $2: 263$ \\
\hline \multirow[t]{3}{*}{ C113A1 } & $\mathrm{AA}$ & T-3001 Zone A1 & DEV1:N7:37 & $1 \mathrm{MO}$ & ADD & $2: 2642: 365$ \\
\hline & & & & & GRT & $2: 2652: 330$ \\
\hline & & & & & LES & $2: 330$ \\
\hline
\end{tabular}




\begin{tabular}{|c|c|c|c|c|c|c|}
\hline & & & & & MOV & $2: 265$ \\
\hline \multirow[t]{4}{*}{ C113A2 } & AA & T-3001 Zone A2 & DEV1:N7:38 & $1 \mathrm{M} 3$ & ADD & $2: 2662: 366$ \\
\hline & & & & & GRT & $2: 2672: 331$ \\
\hline & & & & & LES & $2: 331$ \\
\hline & & & & & MOV & $2: 267$ \\
\hline \multirow[t]{4}{*}{ C113A3 } & AA & T-3001 Zone A3 & DEV1:N7:39 & $1 \mathrm{M} 6$ & ADD & $2: 2682: 367$ \\
\hline & & & & & GRT & 2:269 2:332 \\
\hline & & & & & LES & $2: 332$ \\
\hline & & & & & MOV & $2: 269$ \\
\hline \multirow[t]{4}{*}{ C113A4 } & AA & T-3001 Zone A4 & DEV1:N7:40 & $1 \mathrm{Mg}$ & ADD & $2: 2702: 368$ \\
\hline & & & & & GRT & $2: 2712: 333$ \\
\hline & & & & & LES & $2: 333$ \\
\hline & & & & & MOV & $2: 271$ \\
\hline \multirow[t]{4}{*}{ C113B1 } & AA & T-3001 Zone B1 & DEV1:N7:41 & $2 A O$ & ADD & $2: 2722: 371$ \\
\hline & & & & & GRT & $2: 2732: 335$ \\
\hline & & & & & LES & $2: 335$ \\
\hline & & & & & MOV & $2: 273$ \\
\hline \multirow[t]{4}{*}{$\mathrm{C} 113 \mathrm{~B} 2$} & $A A$ & T-3001 Zone B2 & DEV1:N7:42 & $2 A 3$ & ADD & $2: 2742: 372$ \\
\hline & & & & & GRT & $2: 2752: 336$ \\
\hline & & & & & LES & $2: 336$ \\
\hline & & & & & MOV & $2: 275$ \\
\hline \multirow[t]{4}{*}{ C113B3 } & AA & T-3001 Zone B3 & DEV1:N7:43 & $2 \mathrm{~A} 6$ & $A D D$ & $2: 2762: 373$ \\
\hline & & & & & GRT & $2: 2772: 337$ \\
\hline & & & & & LES & $2: 337$ \\
\hline & & & & & MOV & $2: 277$ \\
\hline \multirow[t]{4}{*}{ C113B4 } & AA & T-3001 Zone B4 & DEV1:N7:44 & $2 \mathrm{~A} 9$ & ADD & $2: 278$ 2:374 \\
\hline & & & & & GRT & $2: 2792: 338$ \\
\hline & & & & & LES & $2: 338$ \\
\hline & & & & & MOV & $2: 279$ \\
\hline \multirow[t]{4}{*}{$\mathrm{C} 113 \mathrm{C} 1$} & AA & T-3001 Zone C1 & DEV1:N7:45 & 2BO & ADD & $2: 2802: 377$ \\
\hline & & & & & GRT & $2: 2812: 340$ \\
\hline & & & & & LES & $2: 340$ \\
\hline & & & & & MOV & $2: 281$ \\
\hline \multirow[t]{4}{*}{$\mathrm{C} 113 \mathrm{C} 2$} & AA & T-3001 Zone C2 & DEV1:N7:46 & 283 & ADD & $2: 2822: 378$ \\
\hline & & & & & GRT & $2: 2832: 341$ \\
\hline & & & & & LES & $2: 341$ \\
\hline & & & & & MOV & $2: 283$ \\
\hline \multirow[t]{4}{*}{ C113C3 } & AA & T-3001 Zone C3 & DEV1:N7:47 & 286 & $\mathrm{ADD}$ & $2: 2842: 379$ \\
\hline & & & & & GRT & $2: 2852: 342$ \\
\hline & & & & & LES & $2: 342$ \\
\hline & & & & & MOV & $2: 285$ \\
\hline \multirow[t]{4}{*}{$\mathrm{C} 113 \mathrm{C} 4$} & AA & T-3001 Zone C4 & DEV1:N7:48 & 2B9 & ADD & $2: 2862: 380$ \\
\hline & & & & & GRT & $2: 2872: 343$ \\
\hline & & & & & LES & $2: 343$ \\
\hline & & & & & MOV & $2: 287$ \\
\hline \multirow[t]{4}{*}{ C119A } & AA & Zone 119A & DEV1:N7:49 & $2 \mathrm{CO}$ & $A D D$ & $2: 4342: 522$ \\
\hline & & & & & GRT & $2: 4352: 494$ \\
\hline & & & & & LES & $2: 494$ \\
\hline & & & & & MOV & $2: 435$ \\
\hline
\end{tabular}




\begin{tabular}{|c|c|c|c|c|c|c|}
\hline Tag Nam & Tag Type & Description & PLC Device & PLCI/O & Ladder & ic Rungs \\
\hline C119B & $A A$ & Zone 119B & DEV1:N7:50 & $2 \mathrm{C} 3$ & ADD & $2: 4362: 523$ \\
\hline & & & & & GRT & $2: 4372: 495$ \\
\hline & & & & & LES & $2: 495$ \\
\hline & & & & & MOV & $2: 437$ \\
\hline $\mathrm{C} 119 \mathrm{C}$ & $A A$ & Zone $119 \mathrm{C}$ & DEV1:N7:51 & $2 \mathrm{C6}$ & $\mathrm{ADD}$ & $2: 4382: 524$ \\
\hline & & & & & GRT & $2: 4392: 496$ \\
\hline & & & & & LES & $2: 496$ \\
\hline & & & & & MOV & $2: 439$ \\
\hline $\mathrm{C} 120 \mathrm{~A}$ & $A A$ & Zone $120 \mathrm{~A}$ & DEV1:N7:52 & $2 \mathrm{Cg}$ & ADD & $2: 4402: 527$ \\
\hline & & & & & GRT & $2: 4412: 498$ \\
\hline & & & & & LES & $2: 498$ \\
\hline & & & & & MOV & $2: 441$ \\
\hline $\mathrm{C} 120 \mathrm{~B}$ & $A A$ & Zone $120 \mathrm{~B}$ & DEV1:N7:53 & 2DO & ADD & $2: 4422: 528$ \\
\hline & & & & & GRT & $2: 4432: 499$ \\
\hline & & & & & LES & $2: 499$ \\
\hline & & & & & MOV & $2: 443$ \\
\hline $\mathrm{C} 120 \mathrm{C}$ & $A A$ & Zone $120 \mathrm{C}$ & DEV1:N7:54 & 2D3 & $A D D$ & $2: 4442: 529$ \\
\hline & & & & & GRT & $2: 4452: 500$ \\
\hline & & & & & LES & $2: 500$ \\
\hline & & & & & MOV & $2: 445$ \\
\hline$C 121 A$ & $A A$ & Zone $121 \mathrm{~A}$ & DEV1:N7:55 & $2 D 6$ & $A D D$ & $2: 4462: 532$ \\
\hline & & & & & GRT & $2: 4472: 502$ \\
\hline & & & & & LES & $2: 502$ \\
\hline & & & & & MOV & $2: 447$ \\
\hline $\mathrm{C} 121 \mathrm{~B}$ & $A A$ & Zone 121B & DEV1:N7:56 & 2D9 & $A D D$ & $2: 4482: 533$ \\
\hline & & & & & GRT & $2: 4492: 503$ \\
\hline & & & & & LES & $2: 503$ \\
\hline & & & & & MOV & $2: 449$ \\
\hline $\mathrm{C} 121 \mathrm{C}$ & $A A$ & Zone 121C & DEV1:N7:57 & $2 \mathrm{EO}$ & ADD & $2: 4502: 534$ \\
\hline & & & & & GRT & $2: 4512: 504$ \\
\hline & & & & & LES & $2: 504$ \\
\hline & & & & & MOV & $2: 451$ \\
\hline$C 122 A$ & $A A$ & Zone 122A & DEV1:N7:58 & $2 \mathrm{E} 3$ & ADD & $2: 4522: 537$ \\
\hline & & & & & GRT & $2: 4532: 506$ \\
\hline & & & & & LES & $2: 506$ \\
\hline & & & & & MOV & $2: 453$ \\
\hline C122B & $A A$ & Zone 122B & DEV1:N7:59 & $2 \mathrm{E} 6$ & ADD & $2: 4542: 538$ \\
\hline & & & & & GRT & $2: 4552: 507$ \\
\hline & & & & & LES & $2: 507$ \\
\hline & & & & & MOV & $2: 455$ \\
\hline $\mathrm{C} 122 \mathrm{C}$ & $A A$ & Zone $122 \mathrm{C}$ & DEV1:N7:60 & 2E9 & $A D D$ & $2: 4562: 539$ \\
\hline & & & & & GRT & $2: 4572: 508$ \\
\hline & & & & & LES & $2: 508$ \\
\hline & & & & & MOV & $2: 457$ \\
\hline$C 123 A$ & $A A$ & Zone 123A & DEV1:N7:61 & $2 \mathrm{FO}$ & ADD & $2: 4582: 542$ \\
\hline & & & & & GRT & $2: 4592: 510$ \\
\hline & & & & & LES & $2: 510$ \\
\hline & & & & & MOV & $2: 459$ \\
\hline $\mathrm{C} 123 \mathrm{~B}$ & $A A$ & Zone 123B & DEV1:N7:62 & $2 \mathrm{~F} 3$ & $A D D$ & $2: 4602: 543$ \\
\hline
\end{tabular}




\begin{tabular}{|c|c|c|c|c|c|c|}
\hline . & & & & & GRT & $2: 4612: 511$ \\
\hline & & & & & LES & $2: 511$ \\
\hline & & & & & MOV & $2: 461$ \\
\hline C123C & $A A$ & Zone $123 \mathrm{C}$ & DEV1:N7:63 & $2 \mathrm{~F} 6$ & $\mathrm{ADD}$ & $2: 4622: 544$ \\
\hline & & & & & GRT & $2: 4632: 512$ \\
\hline & & & & & LES & $2: 512$ \\
\hline & & & & & MOV & $2: 463$ \\
\hline C124A & AA & Zone $124 \mathrm{~A}$ & DEV1:N7:64 & $2 \mathrm{Fg}$ & $A D D$ & $2: 4642: 547$ \\
\hline & & & & & GRT & $2: 4652: 514$ \\
\hline & & & & & LES & $2: 514$ \\
\hline & & & & & MOV & $2: 465$ \\
\hline C124B & $A A$ & Zone 124B & DEV1:N7:65 & $2 \mathrm{GO}$ & ADD & $2: 4662: 548$ \\
\hline & & & & & GRT & $2: 4672: 515$ \\
\hline & & & & & LES & $2: 515$ \\
\hline & & & & & MOV & $2: 467$ \\
\hline C125A & $A A$ & Zone $125 \mathrm{~A}$ & DEV1:N7:66 & $2 \mathrm{G} 3$ & ADD & $2: 4682: 551$ \\
\hline & & & & & GRT & $2: 4692: 517$ \\
\hline & & & & & LES & $2: 517$ \\
\hline & & & & & MOV & $2: 469$ \\
\hline C125B & AA & Zone 125B & DEV1:N7:67 & $2 \mathrm{G6}$ & ADD & $2: 4702: 552$ \\
\hline & & & & & GRT & $2: 4712: 518$ \\
\hline & & & & & LES & $2: 518$ \\
\hline & & & & & MOV & $2: 471$ \\
\hline $\mathrm{C} 130 \mathrm{~A}$ & $A A$ & Zone $130 \mathrm{~A}$ & DEV1:N7:68 & $2 \mathrm{Gg}$ & ADD & $2: 6142: 707$ \\
\hline & & & & & GRT & $2: 6152: 677$ \\
\hline & & & & & LES & $2: 677$ \\
\hline & & & & & MOV & $2: 615$ \\
\hline $\mathrm{C} 130 \mathrm{~B}$ & AA & Zone $130 \mathrm{~B}$ & DEV1:N7:69 & $2 \mathrm{HO}$ & ADD & $2: 6162: 708$ \\
\hline & & & & & GRT & $2: 6172: 678$ \\
\hline & & & & & LES & $2: 678$ \\
\hline & & & & & MOV & $2: 617$ \\
\hline $\mathrm{C} 130 \mathrm{C}$ & AA & Zone 130 & DEV1:N7:70 & $2 \mathrm{H} 3$ & ADD & $2: 6182: 709$ \\
\hline & & & & & GRT & $2: 6192: 679$ \\
\hline & & & & & LES & $2: 679$ \\
\hline & & & & & MOV & $2: 619$ \\
\hline C131A & AA & Zone 131A & DEV1:N7:71 & $2 \mathrm{H} 6$ & ADD & $2: 6202: 712$ \\
\hline & & & & & GRT & $2: 6212: 681$ \\
\hline & & & & & LES & $2: 681$ \\
\hline & & & & & MOV & $2: 621$ \\
\hline C131B & $A A$ & Zone 131B & DEV1:N7:72 & $2 \mathrm{Hg}$ & $A D D$ & $2: 6222: 713$ \\
\hline & & & & & GRT & $2: 6232: 682$ \\
\hline & & & & & LES & $2: 682$ \\
\hline & & & & & MOV & $2: 623$ \\
\hline $\mathrm{C} 131 \mathrm{C}$ & AA & Zone $131 \mathrm{C}$ & DEV1:N7:73 & 210 & $A D D$ & $2: 6242: 714$ \\
\hline & & & & & GRT & $2: 6252: 683$ \\
\hline & & & & & LES & $2: 683$ \\
\hline & & & & & MOV & $2: 625$ \\
\hline C132A & AA & Zone $132 \mathrm{~A}$ & DEV1:N7:74 & 213 & ADD & $2: 6262: 717$ \\
\hline & & & & & GRT & $2: 6272: 685$ \\
\hline
\end{tabular}




\begin{tabular}{|c|c|c|c|c|c|c|}
\hline & & & & & LES & $2: 685$ \\
\hline & & & & & MOV & $2: 627$ \\
\hline \multirow[t]{4}{*}{ C132B } & $A A$ & Zone 132B & DEV1:N7:75 & 216 & ADD & $2: 6282: 718$ \\
\hline & & & & & GRT & $2: 6292: 686$ \\
\hline & & & & & LES & $2: 686$ \\
\hline & & & & & MOV & $2: 629$ \\
\hline \multirow[t]{4}{*}{ C133A } & $\mathrm{AA}$ & Zone 133A & DEV1:N7:76 & 219 & ADD & $2: 6302: 721$ \\
\hline & & & & & GRT & $2: 6312: 688$ \\
\hline & & & & & LES & $2: 688$ \\
\hline & & & & & MOV & $2: 631$ \\
\hline \multirow[t]{4}{*}{ C133B } & $A A$ & Zone 133B & DEV1:N7:77 & $2 \mathrm{JO}$ & $A D D$ & $2: 6322: 722$ \\
\hline & & & & & GRT & $2: 6332: 689$ \\
\hline & & & & & LES & $2: 689$ \\
\hline & & & & & MOV & $2: 633$ \\
\hline \multirow[t]{4}{*}{$\mathrm{C} 134 \mathrm{~A}$} & $A A$ & Zone 134A & DEV1:N7:78 & $2 \mathrm{~J} 3$ & ADD & $2: 6342: 725$ \\
\hline & & & & & GRT & $2: 6352: 691$ \\
\hline & & & & & LES & $2: 691$ \\
\hline & & & & & MOV & $2: 635$ \\
\hline \multirow[t]{4}{*}{ C134B } & $A A$ & Zone 134B & DEV1:N7:79 & $2 \sqrt{6} 6$ & ADD & $2: 6362: 726$ \\
\hline & & & & & GRT & $2: 6372: 692$ \\
\hline & & & & & LES & $2: 692$ \\
\hline & & & & & MOV & $2: 637$ \\
\hline \multirow[t]{4}{*}{ C140A } & $A A$ & Zone 140A & DEV1:N7:80 & $2 \mathrm{Jg}$ & ADD & $2: 6382: 729$ \\
\hline & & & & & GRT & $2: 6392: 694$ \\
\hline & & & & & LES & $2: 694$ \\
\hline & & & & & MOV & $2: 639$ \\
\hline \multirow[t]{4}{*}{ C140B } & $\mathrm{AA}$ & Zone 140B & DEV1:N7:81 & $2 K 0$ & ADD & $2: 6402: 730$ \\
\hline & & & & & GRT & $2: 6412: 695$ \\
\hline & & & & & LES & $2: 695$ \\
\hline & & & & & MOV & $2: 641$ \\
\hline \multirow[t]{4}{*}{ C140C } & $A A$ & Zone $140 \mathrm{C}$ & DEV1:N7:82 & $2 K 3$ & ADD & $2: 6422: 731$ \\
\hline & & & & & GRT & $2: 6432: 696$ \\
\hline & & & & & LES & $2: 696$ \\
\hline & & & & & MOV & $2: 643$ \\
\hline \multirow[t]{4}{*}{ C141A } & $\mathrm{AA}$ & Zone 141A & DEV1:N7:83 & $2 \mathrm{~K} 6$ & ADD & $2: 6442: 734$ \\
\hline & & & & & GRT & $2: 6452: 698$ \\
\hline & & & & & LES & $2: 698$ \\
\hline & & & & & MOV & $2: 645$ \\
\hline \multirow[t]{4}{*}{ C141B } & $\mathrm{AA}$ & Zone 141B & DEV1:N7:84 & $2 \mathrm{Kg}$ & ADD & $2: 6462: 735$ \\
\hline & & & & & GRT & $2: 6472: 699$ \\
\hline & & & & & LES & $2: 699$ \\
\hline & & & & & MOV & $2: 647$ \\
\hline \multirow[t]{4}{*}{$\mathrm{C}_{141 \mathrm{C}}$} & $\mathrm{AA}$ & Zone 141C & DEV1:N7:85 & 2LO & ADD & $2: 6482: 736$ \\
\hline & & & & & GRT & $2: 6492: 700$ \\
\hline & & & & & LES & $2: 700$ \\
\hline & & & & & MOV & $2: 649$ \\
\hline \multirow[t]{3}{*}{ C142A } & $\mathrm{AA}$ & Zone $142 \mathrm{~A}$ & DEV1:N7:86 & $2 \mathrm{~L} 3$ & ADD & $2: 6502: 739$ \\
\hline & & & & & GRT & $2: 6512: 702$ \\
\hline & & & & & LES & $2: 702$ \\
\hline
\end{tabular}


Tag Name Tag Type Description PLCDevice PLC I/O Ladder Logic Rungs

$\begin{array}{llllll} & & & \text { MOV } & 2: 651 \\ \text { C142B AA : Zone 142B } & \text { DEV1:N7:87 } & \text { 2L.6 } & \text { ADD } & 2: 6522: 740 \\ & & & \text { GRT } & 2: 6532: 703 \\ & & & \text { LES } & 2: 703 \\ & & & \text { MOV } & 2: 653\end{array}$

\begin{tabular}{|c|c|c|c|c|c|c|c|c|c|c|c|}
\hline Address & $\begin{array}{l}\text { Symbol } \\
\text { Inatre }\end{array}$ & ction & $\begin{array}{l}\text { Commer } \\
\text { File }\end{array}$ & $\begin{array}{l}\text { int } \\
\text { Number }\end{array}$ & :Rung & Number & & & & & \\
\hline$N 7: 88$ & col & & & & & & & & & & \\
\hline & -ADD - & $2: 153$ & $2: 153$ & $2: 154$ & $2: 154$ & $2: 155$ & $2: 155$ & $2: 156$ & $2: 156$ & $2: 159$ & $2: 159$ \\
\hline & & $2: 160$ & $2: 160$ & $2: 161$ & $2: 161$ & $2: 162$ & $2: 162$ & $2: 165$ & $2: 165$ & $2: 166$ & $2: 166$ \\
\hline & & $2: 167$ & $2: 167$ & $2: 168$ & $2: 168$ & 21171 & $2: 171$ & $2: 172$ & $2: 172$ & $2: 173$ & 2.173 \\
\hline & & $2: 174$ & $2: 174$ & $2: 177$ & $2: 177$ & $2: 178$ & $2: 178$ & $2: 179$ & $2: 179$ & $2: 180$ & $2: 180$ \\
\hline & & $2: 183$ & $2: 183$ & $2: 184$ & $2: 184$ & $2: 185$ & $2: 185$ & $2: 186$ & $2: 186$ & $2: 347$ & $2: 347$ \\
\hline & & $2: 348$ & $2: 348$ & $2: 349$ & $2: 349$ & $2: 350$ & $2: 350$ & $2: 353$ & $2: 353$ & $2: 354$ & $2: 354$ \\
\hline & & $2: 355$ & $2: 355$ & $2: 356$ & $2: 356$ & $2: 359$ & $2: 359$ & $2: 360$ & $2: 360$ & $2: 361$ & $2: 361$ \\
\hline & & $2: 362$ & $2: 362$ & $2: 365$ & $2: 365$ & $2: 366$ & $2: 366$ & $2: 367$ & $2: 367$ & $2: 368$ & $2: 368$ \\
\hline & & $2: 371$ & $2: 371$ & $2: 372$ & $2: 372$ & $2: 373$ & $2: 373$ & $2: 374$ & $2: 374$ & $2: 377$ & $2: 377$ \\
\hline & & $2: 378$ & $2: 378$ & $2: 379$ & $2: 379$ & 21380 & $2: 380$ & 21522 & $2: 522$ & $2: 523$ & $2: 523$ \\
\hline & & $2: 524$ & $2: 524$ & $2: 527$ & $2: 527$ & $2: 528$ & $2: 528$ & $2: 529$ & $2: 529$ & $2: 532$ & $2: 532$ \\
\hline & & $2: 533$ & $2: 533$ & $2: 534$ & $2: 534$ & $2: 537$ & $2: 537$ & $2: 538$ & $2: 538$ & 2,539 & $2: 539$ \\
\hline & & $2: 542$ & $2: 542$ & $2: 543$ & $2: 543$ & $2: 544$ & $2: 544$ & $2: 547$ & $2: 547$ & $2: 548$ & $2: 548$ \\
\hline & & $2: 551$ & $2: 551$ & 2,552 & $2: 552$ & $2: 707$ & $2: 707$ & $2: 708$ & $2: 708$ & $2: 709$ & $2: 709$ \\
\hline & & $2: 712$ & $2: 712$ & $2: 713$ & $2: 713$ & $2: 714$ & $2: 714$ & $2: 717$ & $2: 717$ & $2: 718$ & $2: 718$ \\
\hline & & $2: 721$ & $2: 721$ & $2: 722$ & $2: 722$ & $2: 725$ & $2: 725$ & $2: 726$ & $2: 726$ & $2: 729$ & $2: 729$ \\
\hline & & $2: 730$ & $2: 730$ & $2: 731$ & $2: 731$ & $2: 734$ & $2: 734$ & $2: 735$ & $2: 735$ & $2: 736$ & $2: 736$ \\
\hline & & $2: 739$ & $2: 739$ & $2: 740$ & $2: 740$ & & & & & & \\
\hline & -CLR- & $\begin{array}{l}2: 152 \\
2: 370\end{array}$ & $\begin{array}{l}2: 158 \\
2: 376\end{array}$ & $\begin{array}{l}2: 164 \\
2: 521\end{array}$ & $\begin{array}{l}2: 170 \\
2: 526\end{array}$ & $\begin{array}{l}2: 176 \\
2: 531\end{array}$ & $\begin{array}{l}2: 182 \\
2: 536\end{array}$ & $\begin{array}{l}2: 346 \\
2: 541\end{array}$ & $\begin{array}{l}2: 352 \\
2: 546\end{array}$ & $\begin{array}{l}2: 358 \\
2: 706\end{array}$ & $\begin{array}{l}2: 364 \\
2: 711\end{array}$ \\
\hline & & $2: 716$ & $2: 720$ & $2: 724$ & $2: 728$ & $2: 733$ & $2: 738$ & & & & \\
\hline & -DIV- & $2: 157$ & $2: 163$ & $2: 169$ & $2: 175$ & $2: 181$ & $2: 187$ & $2: 351$ & $2: 357$ & $2: 363$ & $2: 369$ \\
\hline & & $2: 375$ & $2: 381$ & 28525 & $2: 530$ & $2: 535$ & $2: 540$ & $2: 545$ & $2: 549$ & $2: 553$ & $2: 710$ \\
\hline & & & $2: 719$ & $2: 723$ & $2: 727$ & $2: 732$ & $2: 737$ & $2: 741$ & & & \\
\hline
\end{tabular}

-MOV- 2:550 


\begin{tabular}{|c|c|c|c|c|c|c|}
\hline \multirow[t]{5}{*}{ AV110A } & \multirow[t]{5}{*}{$\mathrm{Al}$} & \multirow[t]{5}{*}{ Tank 3002A Average } & \multirow[t]{5}{*}{ DEV1:N7:91 } & \multirow[t]{5}{*}{$2 \mathrm{MOO}$} & $A D D$ & $2: 120$ \\
\hline & & & & & DIV & $2: 157$ \\
\hline & & & & & MOV & $2: 42: 42: 190$ \\
\hline & & & & & PID & 2:195 \\
\hline & & & & & SUB & $2: 120$ \\
\hline \multirow[t]{5}{*}{$A V 110 B$} & \multirow[t]{5}{*}{$\mathrm{Al}$} & \multirow[t]{5}{*}{ Tank 3002B Average } & \multirow[t]{5}{*}{ DEV1:N7:92 } & \multirow[t]{5}{*}{$2 \mathrm{M} 01$} & ADD & $2: 125$ \\
\hline & & & & & DIV & $2: 163$ \\
\hline & & & & & MOV & $2: 52: 52: 198$ \\
\hline & & & & & PID & $2: 203$ \\
\hline & & & & & SUB & $2: 125$ \\
\hline \multirow[t]{5}{*}{ AV110C } & \multirow[t]{5}{*}{$\mathrm{Al}$} & \multirow[t]{5}{*}{ Tank $3002 \mathrm{C}$ Average } & \multirow[t]{5}{*}{ DEV1:N7:93 } & \multirow[t]{5}{*}{$2 \mathrm{MO} 2$} & ADD & $2: 130$ \\
\hline & & & & & DIV & $2: 169$ \\
\hline & & & & & MOV & $2: 62: 62: 206$ \\
\hline & & & & & PID & $2: 211$ \\
\hline & & & & & SUB & $2: 130$ \\
\hline \multirow{5}{*}{ AV111A } & \multirow[t]{5}{*}{ Al } & \multirow{5}{*}{ Tank 3003A Average } & \multirow{5}{*}{ DEV1:N7:94 } & \multirow[t]{5}{*}{$2 \mathrm{MO3}$} & $\mathrm{ADD}$ & $2: 135$ \\
\hline & & & & & DIV & $2: 175$ \\
\hline & & & & & MOV & $2: 72: 72: 214$ \\
\hline & & & & & PID & $2: 219$ \\
\hline & & & & & SUB & $2: 135$ \\
\hline AV111B & $\mathrm{Al}$ & Tank 3003B Average & DEV1:N7:95 & $2 \mathrm{MO4}$ & $\mathrm{ADD}$ & $2: 140$ \\
\hline & & & & & DIV & $2: 181$ \\
\hline & & & & & MOV & $2: 8 \quad 2: 82: 222$ \\
\hline & & & & & PID & $2: 227$ \\
\hline & & & & & SUB & $2: 140$ \\
\hline AV111C & Al & Tank $3003 \mathrm{C}$ Average & DEV1:N7:96 & $2 \mathrm{MO5}$ & $\mathrm{ADD}$ & $2: 145$ \\
\hline & & & & & DIV & $2: 187$ \\
\hline & & & & & MOV & $2: 92: 92: 231$ \\
\hline & & & & & PID & $2: 236$ \\
\hline & & & & & SUB & $2: 145$ \\
\hline AV112A & $\mathrm{Al}$ & Tank 3004A Average & DEV1:N7:97 & $2 M 06$ & ADD & $2: 314$ \\
\hline & & & & & DIV & $2: 351$ \\
\hline & & & & & MOV & $2: 102: 102: 384$ \\
\hline & & & & & PID & $2: 389$ \\
\hline & & & & & SUB & $2: 314$ \\
\hline$A V 112 B$ & $\mathrm{Al}$ & Tank 3004B Average & DEV1:N7:98 & $2 \mathrm{Mo7}$ & $\mathrm{ADD}$ & $2: 319$ \\
\hline & & & & & DIV & $2: 357$ \\
\hline & & & & & MOV & $2: 112: 112: 392$ \\
\hline & & & & & PID & $2: 397$ \\
\hline & & & & & SUB & $2: 319$ \\
\hline $\mathrm{AV} 112 \mathrm{C}$ & Al & Tank $3004 \mathrm{C}$ Average & DEV1:N7:99 & $2 \mathrm{M} 08$ & ADD & $2: 324$ \\
\hline & & & & & DIV & $2: 363$ \\
\hline & & & & & MOV & $2: 122: 122: 400$ \\
\hline & & & & & PID & $2: 405$ \\
\hline & & & & & SUB & $2: 324$ \\
\hline AV113A & $\mathrm{Al}$ & Tank 3001A Average & DEV1:N7:100 & $2 \mathrm{MOg}$ & ADD & $2: 329$ \\
\hline & & & & & DIV & $2: 369$ \\
\hline & & & & & MOV & $2: 132: 132: 408$ \\
\hline & & & & & PID & $2: 413$ \\
\hline & & & & & SUB & $2: 329$ \\
\hline AV113B & $\mathrm{Al}$ & Tank 3001B Average & DEV1:N7:101 & $2 \mathrm{M} 10$ & $A D D$ & $2: 334$ \\
\hline
\end{tabular}




\begin{tabular}{|c|c|c|c|c|c|c|}
\hline & & & & & DIV & $2: 375$ \\
\hline & & & & & MOV & $2: 142: 142: 416$ \\
\hline & & & & & PID & $2: 421$ \\
\hline & & & & & SUB & $2: 334$ \\
\hline AV $113 \mathrm{C}$ & $\mathrm{Al}$ & Tank 3001C Average & DEV1:N7:102 & $2 M 11$ & ADD & $2: 339$ \\
\hline & & & & & DIV & $2: 381$ \\
\hline & & & & & MOV & $2: 152: 152: 425$ \\
\hline & & & & & PID & $2: 430$ \\
\hline & & & & & SUB & $2: 339$ \\
\hline AV119 & $\mathrm{Al}$ & TY-119 Average & DEV1:N7:103 & $2 \mathrm{M} 12$ & ADD & $2: 493$ \\
\hline & & & & & DIV & $2: 525$ \\
\hline & & & & & MOV & $2: 162: 162: 556$ \\
\hline & & & & & PID & $2: 561$ \\
\hline & & & & & SUB & $2: 493$ \\
\hline AV120 & $\mathrm{Al}$ & TY-120 Average & DEV1:N7:104 & $2 \mathrm{M} 13$ & $A D D$ & $2: 497$ \\
\hline & & & & & DIV & $2: 530$ \\
\hline & & & & & MOV & $2: 172: 172: 564$ \\
\hline & & & & & PID & $2: 569$ \\
\hline & & & & & SUB & $2: 497$ \\
\hline AV121 & $\mathrm{Al}$ & TY-121 Average & DEV1:N7:105 & $2 \mathrm{M} 14$ & ADD & $2: 501$ \\
\hline & & & & & DIV & $2: 535$ \\
\hline & & & & & MOV & $2: 182: 182: 572$ \\
\hline & & & & & PID & $2: 577$ \\
\hline & & & & & SUB & $2: 501$ \\
\hline AV122 & $\mathrm{Al}$ & TY-122 Average & DEV1:N7:106 & $2 \mathrm{M} 15$ & $A D D$ & $2: 505$ \\
\hline & & & & & DIV & $2: 540$ \\
\hline & & & & & MOV & $2: 192: 192: 580$ \\
\hline & & & & & PID & $2: 585$ \\
\hline & & & & & SUB & $2: 505$ \\
\hline AV123 & $\mathrm{Al}$ & TY-123 Average & DEV1:N7:107 & $2 \mathrm{M} 16$ & ADD & $2: 509$ \\
\hline & & & & & DIV & $2: 545$ \\
\hline & & & & & MOV & $2: 202: 202: 588$ \\
\hline & & & & & PID & $2: 593$ \\
\hline & & & & & suB & $2: 509$ \\
\hline AV124 & $\mathrm{Al}$ & TY-124 Average & DEV1:N7:108 & $2 \mathrm{M} 17$ & ADD & $2: 513$ \\
\hline & & & & & DIV & $2: 549$ \\
\hline & & & & & MOV & 2:21 2:21 2:596 \\
\hline & & & & & PID & $2: 601$ \\
\hline & & & & & SUB & $2: 513$ \\
\hline AV125 & Al & TY-125 Average & DEV1:N7:109 & $2 \mathrm{M} 18$ & ADD & $2: 516$ \\
\hline & & & & & DIV & $2: 553$ \\
\hline & & & & & MOV & $2: 22$ 2:22 2:605 \\
\hline & & & & & PID & $2: 610$ \\
\hline & & & & & SUB & $2: 516$ \\
\hline$A V 130$ & $\mathrm{Al}$ & TY-130 Average & DEV1:N7:110 & $2 M 19$ & ADD & $2: 676$ \\
\hline & & & & & DIV & $2: 710$ \\
\hline & & & & & MOV & $2: 232: 232: 744$ \\
\hline & & & & & PID & $2: 749$ \\
\hline & & & & & SUB & $2: 676$ \\
\hline AV131 & $\mathrm{Al}$ & TY-131 Average & DEV1:N7:111 & $2 \mathrm{M} 20$ & ADD & $2: 680$ \\
\hline & & & & & DIV & $2: 715$ \\
\hline
\end{tabular}




\begin{tabular}{|c|c|c|c|c|c|c|}
\hline & & & & & MOV & $2: 242: 242: 752$ \\
\hline & & & & & PID & $2: 757$ \\
\hline & & & & & SUB & $2: 680$ \\
\hline \multirow[t]{5}{*}{ AV132 } & Al & TY-132 Average & DEV1:N7:112 & $2 M 21$ & ADD & $2: 684$ \\
\hline & & & & & DIV & $2: 719$ \\
\hline & & & & & MOV & $2: 252: 252: 760$ \\
\hline & & & & & PID & $2: 765$ \\
\hline & & & & & SUB & $2: 684$ \\
\hline \multirow[t]{5}{*}{ AV133 } & $\mathrm{Al}$ & TY-133 Average & DEV1:N7:113 & $2 \mathrm{M} 22$ & $A D D$ & $2: 687$ \\
\hline & & & & & DIV & $2: 723$ \\
\hline & & & & & MOV & $2: 26 \quad 2: 26 \quad 2: 768$ \\
\hline & & & & & PID & $2: 773$ \\
\hline & & & & & SUB & $2: 687$ \\
\hline \multirow[t]{5}{*}{$A V 134$} & Al & TY-134 Average & DEV1:N7:114 & $2 \mathrm{M} 23$ & ADD & $2: 690$ \\
\hline & & & & & DIV & $2: 727$ \\
\hline & & & & & MOV & $2: 272: 272: 776$ \\
\hline & & & & & $\mathrm{PID}$ & $2: 781$ \\
\hline & & & & & SUB & $2: 690$ \\
\hline \multirow[t]{5}{*}{ AV140 } & Al & Zone 140 Average & DEV1:N7:115 & $2 \mathrm{M} 24$ & ADD & $2: 693$ \\
\hline & & & & & DIV & $2: 732$ \\
\hline & & & & & MOV & $2: 28$ 2:28 2:784 \\
\hline & & & & & PID & $2: 789$ \\
\hline & & & & & SUB & $2: 693$ \\
\hline \multirow[t]{5}{*}{ AV141 } & $\mathrm{Al}$ & Zone 141 Average & DEV1:N7:116 & $2 \mathrm{M} 25$ & ADD & $2: 697$ \\
\hline & & & & & DIV & $2: 737$ \\
\hline & & & & & MOV & $2: 292: 292: 792$ \\
\hline & & & & & PID & $2: 797$ \\
\hline & & & & & SUB & $2: 697$ \\
\hline \multirow[t]{5}{*}{ AV142 } & $\mathrm{Al}$ & Zone 142 Average & DEV1:N7:117 & $2 \mathrm{M} 26$ & ADD & $2: 701$ \\
\hline & & & & & DIV & $2: 741$ \\
\hline & & & & & MOV & $2: 302: 302: 801$ \\
\hline & & & & & PID & $2: 806$ \\
\hline & & & & & SUB & $2: 701$ \\
\hline
\end{tabular}




\begin{tabular}{|c|c|c|c|c|c|c|}
\hline & DO & PID MODE SELECT & DEV1:N7:118 & & CLR & $3: 23$ \\
\hline A_MRD110A & & ZONE 110A AUTO/MAN. & DEV1:N7:118/0 & $2 \mathrm{MOO}$ & $-] /[-$ & $2: 193$ \\
\hline \multirow[t]{2}{*}{ ONRD110A } & DO & PID SELECT ON/OFF & DEV1:N7:118/1 & & & $2: 820$ \\
\hline & & & & & & $2: 810$ \\
\hline A_MRD110B & DO & ZONE 1108 AUTO/MAN. & DEV1:N7:118/2 & $2 \mathrm{M} 01$ & & $2: 201$ \\
\hline \multirow[t]{2}{*}{ ONRD110B } & DO & PID SELECT ON/OFF & DEV1:N7:118/3 & & & $2: 820$ \\
\hline & & & & & & $2: 810$ \\
\hline MRD110C & DO & ZONE $110 \mathrm{C}$ AUTO/MAN. & DEV1:N7:118:4 & $2 \mathrm{MO} 2$ & & $2: 209$ \\
\hline \multirow[t]{2}{*}{ ONRD110C } & DO & PID SELECT ON/OFF & DEV1:N7:118:5 & & & $2: 820$ \\
\hline & & & & & & $2: 810$ \\
\hline MRD111A & DO & ONE 111A AUTO/MAN. & DEV1:N7:118:6 & $2 \mathrm{MO3}$ & & $2: 217$ \\
\hline \multirow[t]{2}{*}{ ONRD111A } & DO & PID SELECT ON/OFF & DEV1:N7:118:7 & & & $: 822$ \\
\hline & & & & & - & 811 \\
\hline A_MRD111B & DO & ZONE 111B AUTO/MAN. & DEV1:N7:118:8 & 2M04 & & $2: 225$ \\
\hline \multirow[t]{2}{*}{ ONRD111B } & DO & PID SELEC & DEV1:N7:118:9 & & & $2: 822$ \\
\hline & & & & & & $2: 811$ \\
\hline A_MRD111C & DO & ZONE 111C AUTO/MAN. & DEV1:N7:118:10 & 2M05 & & $2: 234$ \\
\hline \multirow[t]{2}{*}{ ONRD111C } & DO & PID SELECT ON/OFF & DEV1:N7:118:11 & & & $2: 822$ \\
\hline & & & & & & $2: 811$ \\
\hline A_MRD112A & DO & JTO/MAN. & 18:12 & 2M06 & & $2: 387$ \\
\hline \multirow[t]{2}{*}{ ONRD112A } & DO & PID SELECT ON/OFF & DEV1:N7:118:13 & & & $2: 824$ \\
\hline & & & & & & $2: 812$ \\
\hline A_MRD112B & DO & ZONE 112 & 18:14 & $2 \mathrm{M} 07$ & & $2: 395$ \\
\hline \multirow[t]{3}{*}{ ONRD112B } & DO & PID SELECT ON/OFF & DEV1:N7:118:15 & & & $2: 824$ \\
\hline & & & & & $-(U)-$ & $2: 812$ \\
\hline & & DE & DEV & & 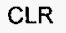 & $3: 24$ \\
\hline MRD112C & DO & /MAN. & DEV & 2M08 & & $2: 403$ \\
\hline \multirow[t]{2}{*}{ ONRD112C } & DO & PID SELECT & DEV1:N7:119:1 & & & $2: 824$ \\
\hline & & & & & )- & $2: 812$ \\
\hline \multirow{3}{*}{$\begin{array}{l}\text { A_MRD113A } \\
\text { ONRD113A }\end{array}$} & DO & MAN. & DEV & $2 \mathrm{MO9}$ & -1 & $2: 411$ \\
\hline & DO & PID SELEC & DEV & & & $2: 826$ \\
\hline & & & & & & $2: 813$ \\
\hline A_MRD113B & DO & /MAN. & DEV & 2M10 & $-] /[-$ & $2: 419$ \\
\hline \multirow[t]{2}{*}{ ONRD113B } & DO & PID SELECT & DEV1:N7:119:5 & & $-][-$ & $2: 826$ \\
\hline & & & & & $-(U)-$ & $2: 813$ \\
\hline A_MRD113C & DO & IMAN. & & $2 \mathrm{M} 11$ & $-] /[-$ & $2: 428$ \\
\hline \multirow[t]{2}{*}{ ONRD113C } & DO & PID SELECT ON/OFF & DEV1:N7:119:7 & & $-][-$ & $2: 826$ \\
\hline & & & & & $-(U)$ & $2: 813$ \\
\hline 119 & DO & V. & & $2 \mathrm{M}$ & $-] /[-$ & $2: 559$ \\
\hline NR & DO & LEC & DEV & & $-] /[-$ & $2: 558$ \\
\hline MRD120 & DO & TY & DEV & $2 M 13$ & $-] /[-$ & $2: 567$ \\
\hline ONR & DO & PIL & $: 11$ & & $-] /[-$ & $2: 566$ \\
\hline A_MRD121 & DO & TY & $9: 12$ & 2M14 & $-] /[$ & $2: 575$ \\
\hline ONRD121 & DO & PID & 13 & & $-] /[-$ & $2: 574$ \\
\hline A_MRD122 & DO & TY-122 Al & 119:14 & 2M15 & $-] /[-$ & $2: 583$ \\
\hline \multirow[t]{2}{*}{ ONRD122 } & DO & PID SELECT ON/OFF & DEV1:N7:119:15 & & $-] /[-$ & $2: 582$ \\
\hline & & PID MODE SELECT & & & CLR & $3: 25$ \\
\hline A_MRD123. & DO & TY-123 AUTO/MAN. & DEV1:N7:120:0 & 2M16 & $-1 /[-$ & $2: 591$ \\
\hline ONRD123 & DO & PID SELECT ON/OFF & DEV1:N7:120:1 & & $-] /[-$ & $2: 590$ \\
\hline A_MRD124 & DO & TY-124 AUTO/MAN. & DEV1:N7:120:2 & 2M17 & $-] /[-$ & $2: 599$ \\
\hline ONRD124 & DO & ELECT ON/OFF & DEV1 & & $-1 /[-$ & $2: 598$ \\
\hline A_MRD125 & DO & TY-125 AUTO/MAN. & DEV1:N7:120:4 & $2 \mathrm{M} 18$ & $-] /[-$ & $2: 608$ \\
\hline
\end{tabular}




\begin{tabular}{|c|c|c|c|c|c|c|}
\hline ONRD125 & DO & PID SELECT ON/OFF & DEV1:N7:120:5 & & $-1 /[-$ & 2:607 \\
\hline A_MRD130 & DO & TY-130 AUTO/MAN. & DEV1:N7:120:6 & $2 \mathrm{M} 19$ & & $2: 747$ \\
\hline ONRD130 & DO & PID SELECT ON/OFF & DEV1:N7:120:7 & & $-] /[-$ & $2: 746$ \\
\hline A_MRD131 & DO & TY-131 AUTO/MAN. & DEV1:N7:120:8 & $2 \mathrm{M} 20$ & $-] /[-$ & $2: 75$ \\
\hline ONRD131 & DO & PID SELECT ON/OFF & DEV1:N7:120:9 & & $-] /[-$ & $2 ; 7$ \\
\hline A_MRD132 & DO & TY-132 AUTO/MAN. & DEV1:N7:120:10 & $2 \mathrm{M} 21$ & $-] /[-$ & $2: 763$ \\
\hline ONRD132 & DO & PID SELECT ON/OFF & DEV1:N7:120:11 & & & $2: 762$ \\
\hline A_MRD133 & DO & TY-133 AUTO/MAN. & DEV1:N7:120:12 & $2 \mathrm{M} 22$ & & $2: 77$ \\
\hline ONRD133 & DO & PID SELECT ON/OFF & DEV1:N7:120:13 & & & $2: 77$ \\
\hline A_MRD134 & DO & TY-134 AUTO/MAN. & DEV1:I & $2 \mathrm{M} 23$ & & $2: 77$ \\
\hline \multirow[t]{2}{*}{ ONRD134 } & DO & PID SELECT ON/OFF & DEV1:N7:120:15 & & & $2: 77$ \\
\hline & & PID MODE SELECT & DEV1: & & CLR & $3: 26$ \\
\hline A_MRD140 & DO & TY-140 AUTO/MAN. & DEV1:N7:121:0 & $2 \mathrm{M} 24$ & $-\sqrt{\prime}$ & $2: 787$ \\
\hline ONRD140 & DO & PID SELECT ON/OFF & DEV1:N7:121:1 & & $-] /$ & $2: 78$ \\
\hline A_MRD141 & DO & TY-141 AUTO/MAN. & DEV1:N7:121:2 & $2 \mathrm{M} 25$ & $-] /[-$ & $2: 79$ \\
\hline ONRD141 & DO & PID SELECT ON/OFF & DEV1:N7:121:3 & & $-] /[-$ & $2: 79$ \\
\hline A_MRD142 & DO & TY-142 AUTO/MAN. & DEV1:N7:121:4 & $2 \mathrm{M} 26$ & $-] /[-$ & $2: 80$ \\
\hline ONRD142 & DO & PID SELECT ON/OFF & DEV1:N7:121:5 & & $-] /[-$ & $2: 80$ \\
\hline
\end{tabular}




\begin{tabular}{|c|c|c|c|c|c|c|}
\hline SET110A & $\mathrm{AO}$ & TY-110A SET POINT ADJ. & DEV1:N7:122 & $2 \mathrm{MOO}$ & MOV & $2: 194$ \\
\hline SET110B & $\mathrm{AO}$ & TY-110B SET POINT ADJ. & DEV1:N7:123 & $2 \mathrm{M} 01$ & MOV & $2: 202$ \\
\hline SET110C & $\mathrm{AO}$ & TY-110C SET POINT ADJ. & DEV1:N7:124 & $2 \mathrm{MO} 2$ & MOV & $2: 210$ \\
\hline SET111A & $\mathrm{AO}$ & TY-111A SET POINT ADJ. & DEV1:N7:125 & $2 \mathrm{MO3}$ & MOV & $2: 218$ \\
\hline SET111B & $\mathrm{AO}$ & TY-111B SET POINT ADJ. & DEV1:N7:126 & $2 \mathrm{M} 04$ & MOV & $2: 226$ \\
\hline SET111C & $\mathrm{AO}$ & TY-111C SET POINT ADJ. & DEV1:N7:127 & $2 \mathrm{MO5}$ & MOV & $2: 235$ \\
\hline SET112A & $\mathrm{AO}$ & TY-112A SET POINT ADJ. & DEV1:N7:128 & $2 M 06$ & MOV & $2: 388$ \\
\hline SET112B & $\mathrm{AO}$ & TY-112B SET POINT ADJ. & DEV1:N7:129 & 2M07 & MOV & $2: 396$ \\
\hline SET112C & $\mathrm{AO}$ & TY-112C SET POINT ADJ. & DEV1:N7:130 & $2 \mathrm{M} 08$ & MOV & $2: 404$ \\
\hline SET113A & $\mathrm{AO}$ & TY-113A SET POINT ADJ. & DEV1:N7:131 & $2 \mathrm{M} 09$ & MOV & $2: 412$ \\
\hline SET113B & $\mathrm{AO}$ & TY-113B SET POINT ADJ. & DEV1:N7:132 & $2 \mathrm{M} 10$ & MOV & $2: 420$ \\
\hline SET113C & $\mathrm{AO}$ & TY-113C SET POINT ADJ. & DEV1:N7:133 & $2 \mathrm{M} 11$ & MOV & $2: 429$ \\
\hline SET119 & $\mathrm{AO}$ & TY-119 SET POINT ADJ. & DEV1:N7:134 & $2 \mathrm{M} 12$ & MOV & $2: 560$ \\
\hline SET 120 & $\mathrm{AO}$ & TY-120 SET POINT ADJ. & DEV1:N7:135 & $2 \mathrm{M} 13$ & MOV & $2: 568$ \\
\hline SET 121 & $\mathrm{AO}$ & TY-121 SET POINT ADJ. & DEV1:N7:136 & $2 \mathrm{M} 14$ & MOV & $2: 576$ \\
\hline SET122 & $\mathrm{AO}$ & TY-122 SET POINT ADJ. & DEV1:N7:137 & $2 \mathrm{M} 15$ & MOV & $2: 584$ \\
\hline SET123 & $\mathrm{AO}$ & TY-123 SET POINT ADJ. & DEV1:N7:138 & $2 \mathrm{M} 16$ & MOV & $2: 592$ \\
\hline SET124 & $\mathrm{AO}$ & TY-124 SET POINT ADJ. & DEV1:N7:139 & $2 \mathrm{M} 17$ & MOV & $2: 600$ \\
\hline SET125 & $\mathrm{AO}$ & TY-125 SET POINT ADJ. & DEV1:N7:140 & $2 \mathrm{M} 18$ & MOV & $2: 609$ \\
\hline SET130 & $\mathrm{AO}$ & TY-130 SET POINT ADJ & DEV1:N7:141 & $2 M 19$ & MOV & $2: 748$ \\
\hline SET131 & $\mathrm{AO}$ & TY-131 SET POINT ADJ & DEV1:N7:142 & $2 \mathrm{M} 20$ & MOV & $2: 756$ \\
\hline SET132 & $\mathrm{AO}$ & TY-132 SET POINT ADJ & DEV1:N7:143 & $2 \mathrm{M} 21$ & MOV & $2: 764$ \\
\hline SET133 & AO & TY-133 SET POINT ADJ & DEV1:N7:144 & $2 \mathrm{M} 22$ & MOV & $2: 772$ \\
\hline SET134 & $\mathrm{AO}$ & TY-134 SET POINT ADJ & DEV1:N7:145 & $2 M 23$ & MOV & $2: 780$ \\
\hline SET140 & $\mathrm{AO}$ & TY-140 SET POINT ADJ & DEV1:N7:146 & $2 M 24$ & MOV & $2: 788$ \\
\hline SET141 & $\mathrm{AO}$ & TY-141 SET POINT ADJ & DEV1:N7:147 & $2 \mathrm{M} 25$ & MOV & $2: 796$ \\
\hline SET142 & $\mathrm{AO}$ & TY-142 SET POINT ADJ & DEV1:N7:181 & $2 \mathrm{M} 26$ & MOV & $2: 805$ \\
\hline
\end{tabular}

HNF-SD-FF-CSWD-60 REV. 0 Page $5 /$ 


\begin{tabular}{|c|c|c|c|c|c|c|}
\hline D110A1 & DA & $\begin{array}{l}\text { TE-110A1 Value } \\
\text { Out of Range (OOR) }\end{array}$ & DEV1:N7:148/0 & 1Do & $\begin{array}{l}-] /[- \\
-()-\end{array}$ & $\begin{array}{l}2: 153 \\
2: 121\end{array}$ \\
\hline \multirow[t]{2}{*}{ D110A2 } & $\mathrm{DA}$ & TE-110A2 Val. OOR & DEV1:N7:148/1 & $1 \mathrm{D} 3$ & $-] /[-$ & $2: 154$ \\
\hline & & & & & & $2: 122$ \\
\hline \multirow[t]{2}{*}{ D110A3 } & $\mathrm{DA}$ & TE-110A3 Val. OOR & DEV1:N7:148/2 & 1D6 & $-] /[-$ & $2: 155$ \\
\hline & & & & & -()$-$ & $2: 123$ \\
\hline \multirow[t]{2}{*}{ D110A4 } & DA & TE-110A4 Val. OOR & DEV1:N7:148/3 & 1D9 & $-] /[-$ & $2: 156$ \\
\hline & & & & & & $2: 124$ \\
\hline \multirow[t]{2}{*}{ D110B1 } & DA & TE-110B1 Val. OOR & DEV1:N7:148/4 & 1E0 & $-] /[-$ & $2: 159$ \\
\hline & & & & & & $2: 126$ \\
\hline \multirow[t]{2}{*}{ D110B2 } & $D A$ & TE-110B2 Val. OOR & DEV1:N7:148/5 & 1E3 & $-] /[-$ & $2: 160$ \\
\hline & & & & & & $2: 127$ \\
\hline \multirow[t]{2}{*}{ D110B3 } & DA & TE-110B3 Val. OOR & DEV1:N7:148/6 & iE6 & $-] /[-$ & $2: 161$ \\
\hline & & & & & -( & $2: 128$ \\
\hline \multirow[t]{2}{*}{ D110B4 } & DA & TE-110B4 Val. OOR & DEV1:N7:148/7 & $1 \mathrm{E} 9$ & $-] /[-$ & $2: 162$ \\
\hline & & & & & & $2: 129$ \\
\hline \multirow[t]{2}{*}{ D110C1 } & DA & TE-110C1 Val. OOR & DEV1:N7:148/8 & $1 F 0$ & $-] /[-$ & $2: 165$ \\
\hline & & & & & & $2: 131$ \\
\hline \multirow[t]{2}{*}{ D110C2 } & DA & TE-110C2 Val. OOR & DEV1:N7:148/9 & $1 \mathrm{~F} 3$ & $-] /[-$ & $2: 166$ \\
\hline & & & & & -()$-$ & $2: 132$ \\
\hline \multirow[t]{2}{*}{ D110C3 } & DA & TE-110C3 Val. OOR & DEV1:N7:148/10 & $1 \mathrm{~F} 6$ & $-] /[-$ & $2: 167$ \\
\hline & & & & & -()$-$ & $2: 133$ \\
\hline \multirow[t]{2}{*}{ D110C4 } & DA & TE-110C4 Val. OOR & DEV1:N7:148/11 & 1F9 & $-3 /[-$ & $2: 168$ \\
\hline & & & & & -()$-$ & $2: 134$ \\
\hline \multirow[t]{2}{*}{ D111A1 } & DA & TE-111A1 Val. OOR & DEV1:N7:148/12 & $1 \mathrm{GO}$ & $-1 /[-$ & $2: 171$ \\
\hline & & & & & -()$-$ & $2: 136$ \\
\hline \multirow[t]{2}{*}{ D111A2 } & DA & TE-111A2 Val. OOR & DEV1:N7:148/13 & $1 \mathrm{G} 3$ & $-] /[-$ & $2: 172$ \\
\hline & & & & & -()$-$ & $2: 137$ \\
\hline \multirow[t]{2}{*}{ D111A3 } & DA & TE-111A3 Val. OOR & DEV1:N7:148/14 & $1 \mathrm{G6}$ & $-3 /[-$ & $2: 173$ \\
\hline & & & & & -()$-$ & $2: 138$ \\
\hline \multirow[t]{2}{*}{ D111A4 } & DA & TE-111A4 Val. OOR & DEV1:N7:148/15 & $1 \mathrm{Gg}$ & $-3 /[-$ & $2: 174$ \\
\hline & & & & & -()$-$ & $2: 139$ \\
\hline \multirow[t]{2}{*}{ D111B1 } & DA & TE-111B1 Val. OOR & DEV1:N7:149/0 & $1 \mathrm{HO}$ & $-] /[-$ & $2: 177$ \\
\hline & & & & & -() & $2: 141$ \\
\hline \multirow[t]{2}{*}{ D111B2 } & DA & TE-111B2 Val. OOR & DEV1:N7:149/1 & $1 \mathrm{H} 3$ & $-3 /[-$ & $2: 178$ \\
\hline & & & & & -()$-$ & $2: 142$ \\
\hline \multirow[t]{2}{*}{ D111B3 } & DA & TE-111B3 Val. OOR & DEV1:N7:149/2 & $1 \mathrm{H} 6$ & $-] /[-$ & $2: 179$ \\
\hline & & & & & -()$-$ & $2: 143$ \\
\hline \multirow[t]{2}{*}{ D111B4 } & $\mathrm{DA}$ & TE-111B4 Val. OOR & DEV1:N7:149/3 & $1 \mathrm{Hg}$ & $-] /[-$ & $2: 180$ \\
\hline & & & & & -()$-$ & $2: 144$ \\
\hline \multirow[t]{2}{*}{ D111C1 } & DA & TE-111C1 Val. OOR & DEV1:N7:149/4 & 110 & $-] /[-$ & $2: 183$ \\
\hline & & & & & $-(j)$ & $2: 146$ \\
\hline $\mathrm{D} 111 \mathrm{C} 2$ & DA & TE-111C2 Val. OOR & DEV1:N7:149/5 & 113 & $-1 /[-$ & $2: 184$ \\
\hline & & & & & -()$-$ & $2: 147$ \\
\hline D111C3 & DA & TE-111C3 Val. OOR & DEV1:N7:149/6 & 116 & $-] /[-$ & $2: 185$ \\
\hline & & & & & -() & $2: 148$ \\
\hline $\mathrm{D} 111 \mathrm{C} 4$ & DA & TE-111C4 Val. OOR & DEV1:N7:149/7 & 119 & $-] /[-$ & $2: 186$ \\
\hline & & & & & -()$-$ & $2: 149$ \\
\hline D112A1 & DA & TE-112A1 Val. OOR & DEV1:N7:149/8 & 1Jo & $-] /[-$ & $2: 347$ \\
\hline & & & & & -1 & $2: 315$ \\
\hline D112A2 & DA & TE-112A2 Val. OOR & DEV1:N7:149/9 & $1 \sqrt{3}$ & $-] /[-$ & $2: 348$ \\
\hline
\end{tabular}




\begin{tabular}{|c|c|c|c|c|c|c|}
\hline \multirow{3}{*}{ D112A3 } & \multirow[b]{2}{*}{ DA } & \multirow{3}{*}{ TE-112A3 Val. OOR } & \multirow{3}{*}{ DEV1:N7:149/10 } & \multirow{3}{*}{$1 \mathrm{~J} 6$} & \multirow{2}{*}{$\begin{array}{l}-()- \\
-] /[-\end{array}$} & \multirow{2}{*}{$2: 316$} \\
\hline & & & & & & \\
\hline & & & & & & $2: 317$ \\
\hline \multirow[t]{2}{*}{ D112A4 } & \multirow[t]{2}{*}{ DA } & \multirow[t]{2}{*}{ TE-112A4 Val. OOR } & \multirow[t]{2}{*}{ DEV1:N7:149/11 } & \multirow[t]{2}{*}{$1 \mathrm{J9}$} & $-j / /-$ & $2: 350$ \\
\hline & & & & & & $2: 318$ \\
\hline \multirow{2}{*}{ D112B1 } & \multirow[t]{2}{*}{ DA } & \multirow{2}{*}{ TE-112B1 Val. OOR } & \multirow[t]{2}{*}{ DEV1:N7:149/12 } & \multirow[t]{2}{*}{$1 \mathrm{Ko}$} & $-] /[-$ & $2: 353$ \\
\hline & & & & & & $2: 320$ \\
\hline \multirow[t]{2}{*}{$\mathrm{D} 112 \mathrm{~B} 2$} & \multirow[t]{2}{*}{ DA } & \multirow[t]{2}{*}{ TE-112B2 Val. OOR } & \multirow[t]{2}{*}{ DEV1:N7:149/13 } & $1 \mathrm{~K} 3$ & $-] /[-$ & $2: 354$ \\
\hline & & & & & & $2: 321$ \\
\hline D112B3 & DA & TE-112B3 Val. OOR & DEV1:N7:149/14 & $1 \mathrm{~K} 6$ & $-] /[-$ & $2: 355$ \\
\hline & & & & & -()$-$ & $2: 322$ \\
\hline D112B4 & DA & TE-112B4 Val. OOR & DEV1:N7:149/15 & $1 \mathrm{~K} 9$ & $-] /[-$ & $2: 356$ \\
\hline & & & & & -() & $2: 323$ \\
\hline D112C1 & DA & TE-112C1 Val. OOR & DEV1:N7:150/0 & $1 \mathrm{LO}$ & $-] /[-$ & $2: 359$ \\
\hline & & & & & -()$-$ & $2: 325$ \\
\hline $\mathrm{D} 112 \mathrm{C} 2$ & DA & TE-112C2 Val. OOR & DEV1:N7:150/1 & $1 \mathrm{~L} 3$ & $-] /[-$ & $2: 360$ \\
\hline & & & & & & $2: 326$ \\
\hline D112C3 & DA & TE-112C3 Val. OOR & DEV1:N7:150/2 & $1 \mathrm{~L} 6$ & $-] /[-$ & $2: 361$ \\
\hline & & & & & & $2: 327$ \\
\hline D112C4 & DA & TE-112C4 Val. OOR & DEV1:N7:150/3 & $1 \mathrm{Lg}$ & $-] /[-$ & $2: 362$ \\
\hline & & & & & -()$-$ & $2: 328$ \\
\hline D113A1 & DA & TE-113A1 Val. OOR & DEV1:N7:150/4 & $1 \mathrm{MO}$ & $-] /[-$ & $2: 365$ \\
\hline & & & & & -()$-$ & $2: 330$ \\
\hline D113A2 & DA & TE-113A2 Val. OOR & DEV1:N7:150/5 & $1 \mathrm{M} 3$ & $-1 / /-$ & $2: 366$ \\
\hline & & & & & -()$-$ & $2: 331$ \\
\hline D113A3 & $\mathrm{DA}$ & TE-113A3 Val. OOR & DEV1:N7:150/6 & $1 \mathrm{M} 6$ & $-] /[-$ & $2: 367$ \\
\hline & & & & & -()$-$ & $2: 332$ \\
\hline D113A4 & DA & TE-113A4 Val. OOR & DEV1:N7:150/7 & $1 \mathrm{M} 9$ & $-3 / /-$ & $2: 368$ \\
\hline & & & & & -() & $2: 333$ \\
\hline D113B1 & DA & TE-113B1 Val. OOR & DEV1:N7:150/8 & $2 \mathrm{AO}$ & $-3 / 1-$ & $2: 371$ \\
\hline & & & & & $-(1)-$ & 2:335 \\
\hline D113B2 & DA & TE-113B2 Val. OOR & DEV1:N7:150/9 & $2 A 3$ & $-] /[-$ & $2: 372$ \\
\hline & & & & & -()$-$ & $2: 336$ \\
\hline D113B3 & DA & TE-113B3 Val. OOR & DEV1:N7:150/10 & $2 A 6$ & $-] /[-$ & $2: 373$ \\
\hline & & & & & -()$-$ & $2: 337$ \\
\hline D113B4 & DA & TE-113B4 Val. OOR & DEV1:N7:150/11 & $2 \mathrm{~A} 9$ & $-] /[-$ & $2: 374$ \\
\hline & & & & & -() & $2: 338$ \\
\hline D113C1 & DA & TE-113C1 Val. OOR & DEV1:N7:150/12 & $2 \mathrm{BO}$ & $-] /[-$ & $2: 377$ \\
\hline & & & & & -()$-$ & $2: 340$ \\
\hline D113C2 & DA & TE-113C2 Val. OOR & DEV1:N7:150/13 & 2B3 & $-1 /[-$ & $2: 378$ \\
\hline & & & & & -()$-$ & $2: 341$ \\
\hline D113C3 & $\mathrm{DA}$ & TE-113C3 Val. OOR & DEV1:N7:150/14 & 2B6 & $-] /[-$ & $2: 379$ \\
\hline & & & & & -()$-$ & $2: 342$ \\
\hline D113C4 & DA & TE-113C4 Val. OOR & DEV1:N7:150/15 & 289 & $-] /[-$ & $2: 380$ \\
\hline & & & & & -()$-$ & $2: 343$ \\
\hline D119A & DA & TE-119A Val. OOR & DEV1:N7:151/0 & $2 \mathrm{CO}$ & $-1 /[-$ & $2: 522$ \\
\hline & & & & & -()$-$ & $2: 494$ \\
\hline D119B & DA & TE-119B Val. OOR & DEV1:N7:151/1 & $2 \mathrm{C} 3$ & $-] /[-$ & $2: 523$ \\
\hline & & & & & -()$-$ & $2: 495$ \\
\hline D119C & DA & TE-119C Val. OOR & DEV1:N7:151/2 & $2 \mathrm{C} 6$ & $-1 /[-$ & $2: 524$ \\
\hline & & & & & -()$-$ & $2: 496$ \\
\hline
\end{tabular}




\begin{tabular}{|c|c|c|c|c|c|c|}
\hline D120A & DA & TE-120A Val. OOR & DEV1:N7:151/3 & $2 \mathrm{C9}$ & $\begin{array}{l}-] /[- \\
-(1)-\end{array}$ & $\begin{array}{l}2: 527 \\
2: 498\end{array}$ \\
\hline \multirow[t]{2}{*}{ D120B } & DA & TE-120B Val. OOR & DEV1:N7:151/4 & $2 \mathrm{DO}$ & $-] /[-$ & $2: 528$ \\
\hline & & & & & & $2: 499$ \\
\hline \multirow[t]{2}{*}{ D120C } & DA & TE-120C Val. OOR & DEV1:N7:151/5 & 2D3 & $-] /[-$ & $2: 529$ \\
\hline & & & & & -1 & $2: 500$ \\
\hline \multirow[t]{2}{*}{ D121A } & $\mathrm{DA}$ & TE-121A Val. OOR & DEV1:N7:151/6 & 2D6 & $-] /[$ & $2: 532$ \\
\hline & & & & & & $2: 502$ \\
\hline \multirow[t]{2}{*}{ D121B } & $\mathrm{DA}$ & TE-121B Val. OOR & DEV1:N7:151/7 & $2 \mathrm{D} 9$ & $-] /[-$ & $2: 533$ \\
\hline & & & & & & $2: 503$ \\
\hline \multirow[t]{2}{*}{ D121C } & $\mathrm{DA}$ & TE-121C Val. OOR & DEV1:N7:151/8 & 2E0 & $-]$ & $2: 534$ \\
\hline & & & & & - & $2: 504$ \\
\hline D122A & DA & I/ vaiue Uut or Ran & DEVI:N $/ .157 / 9$ & $2 \mathrm{E} 3$ & $-] /[-$ & $2: 537$ \\
\hline \multirow[t]{2}{*}{ D122B } & $\mathrm{DA}$ & T/C Value Out of Ran & DEV1:N7:151/10 & 2E6 & $-] /[-$ & $2: 538$ \\
\hline & & & & & -1 & $2: 507$ \\
\hline \multirow[t]{2}{*}{ D122C } & DA & T/C Value Out of Ran & DEV1:N7:151/11 & $2 \mathrm{E} 9$ & $-] /[-$ & $2: 539$ \\
\hline & & & & & & $2: 508$ \\
\hline \multirow[t]{2}{*}{ D123A } & DA & T/C Value Out of Ran & DEV1:N7:151/12 & $2 \mathrm{FO}$ & -1 & $2: 542$ \\
\hline & & & & & -( & $2: 510$ \\
\hline \multirow[t]{2}{*}{ D123B } & DA & T/C Value Out of Ran & DEV1:N7:151/13 & $2 \mathrm{~F} 3$ & $-] /[-$ & $2: 543$ \\
\hline & & & & & & $2: 511$ \\
\hline \multirow[t]{2}{*}{ D123C } & DA & T/C Value Out of Ran & DEV1:N7:151/14 & $2 \mathrm{~F} 6$ & $-1 /[-$ & $2: 544$ \\
\hline & & & & & -()$-$ & $2: 512$ \\
\hline \multirow[t]{2}{*}{ D124A } & DA & T/C Value Out of Ran & DEV1:N7:151/15 & $2 \mathrm{F9}$ & $-] /[-$ & $2: 547$ \\
\hline & & & & & & $2: 514$ \\
\hline \multirow[t]{2}{*}{ D124B } & DA & T/C Value Out of Ran & DEV1:N7:152/0 & $2 \mathrm{GO}$ & $-] /[-$ & $2: 548$ \\
\hline & & & & & -1 & $2: 515$ \\
\hline \multirow[t]{2}{*}{ D125A } & $\mathrm{DA}$ & $\mathrm{T} / \mathrm{C}$ Value Out of Ran & DEV1:N7:152/1 & $2 \mathrm{G} 3$ & $-1 /[-$ & $2: 551$ \\
\hline & & & & & -()$-$ & $2: 517$ \\
\hline \multirow[t]{2}{*}{ D125B } & $\mathrm{DA}$ & $\mathrm{T} / \mathrm{C}$ Value Out of Ran & DEV1:N7:152/2 & $2 \mathrm{G} 6$ & $-] /[\cdot$ & $2: 552$ \\
\hline & & & & & -()$-$ & $2: 518$ \\
\hline \multirow[t]{2}{*}{ D130A } & DA & T/C Value Out of Ran & DEV1:N7:152/3 & $2 \mathrm{G9}$ & $-1 /[-$ & $2: 707$ \\
\hline & & & & & -()$-$ & $2: 677$ \\
\hline \multirow[t]{2}{*}{$\mathrm{D} 130 \mathrm{~B}$} & DA & $\mathrm{T} / \mathrm{C}$ Value Out of Ran & DEV1:N7:152/4 & $2 \mathrm{HO}$ & $-] /[-$ & $2: 708$ \\
\hline & & & & & ${ }^{-1}$ & $2: 678$ \\
\hline \multirow[t]{2}{*}{ D130C } & DA & $T / C$ Value Out of Ran & DEV1:N7:152/5 & $2 \mathrm{H}_{3}$ & $-] /[-$ & $2: 709$ \\
\hline & & & & & -( & $2: 679$ \\
\hline \multirow[t]{2}{*}{ D131A } & DA & $T / C$ Value Out of Ran & DEV1:N7:152/6 & $2 \mathrm{H} 6$ & $-] /[-$ & 2:712 \\
\hline & & & & & -()$-$ & $2: 681$ \\
\hline \multirow{2}{*}{ D131B } & $\mathrm{DA}$ & T/C Value Out of Ran & DEV1:N7:152/7 & $2 \mathrm{H} 9$ & $-] /[-$ & $2: 713$ \\
\hline & & & & & -( & $2: 682$ \\
\hline D131C & DA & $T / C$ Value Out of Ran & DEV1:N7:152/8 & 210 & $-] /[-$ & $2: 714$ \\
\hline & & & & & -()$-$ & $2: 683$ \\
\hline D132A & DA & $\mathrm{T} / \mathrm{C}$ Value Out of Ran & DEV1:N7:152/9 & $2 / 3$ & $-] /[-$ & $2: 717$ \\
\hline & & & & & -()$-$ & $2: 685$ \\
\hline D132B & DA & $T / C$ Value Out of Ran & DEV1:N7:152/10 & 216 & $-] /[-$ & $2: 718$ \\
\hline & & & & & -()$-$ & $2: 686$ \\
\hline D133A & DA & $T / C$ Value Out of Ran & DEV1:N7:152/11 & 219 & $-] /[-$ & $2: 721$ \\
\hline & & & & & -()$-$ & $2: 688$ \\
\hline D133B & DA & T/C Value Out of Ran & DEV1:N7:152/12 & $2 \mathrm{~J} 0$ & $-1 /[-$ & $2: 722$ \\
\hline
\end{tabular}




\begin{tabular}{|c|c|c|c|c|c|c|}
\hline \multirow{3}{*}{ D134 } & \multirow{3}{*}{ DA } & \multirow{3}{*}{$T / C$ Value Out of Ran } & \multirow{3}{*}{ DEV1:N7:152/13 } & \multirow{3}{*}{$2 \mathrm{~J} 3$} & -()$-$ & $2: 689$ \\
\hline & & & & & $-] /[-$ & $2: 725$ \\
\hline & & & & & & 2:691 \\
\hline \multirow[t]{2}{*}{ D134B } & \multirow[t]{2}{*}{ DA } & \multirow[t]{2}{*}{ T/C Value Out of Ran } & \multirow[t]{2}{*}{ DEV1:N7:152/14 } & \multirow[t]{2}{*}{$2 J 6$} & $-j /$ & $2: 726$ \\
\hline & & & & & & $2: 692$ \\
\hline \multirow[t]{2}{*}{ D140A } & \multirow[t]{2}{*}{ DA } & \multirow[t]{2}{*}{ T/C Value Out of Ran } & \multirow[t]{2}{*}{ DEV1:N7:152/15 } & \multirow[t]{2}{*}{ 2J9 } & $-] / /-$ & $2: 729$ \\
\hline & & & & & & 2:694 \\
\hline \multirow[t]{2}{*}{ D140B } & \multirow[t]{2}{*}{ DA } & \multirow[t]{2}{*}{ T/C Value Out of Ran } & \multirow[t]{2}{*}{ DEV1:N7:153/0 } & \multirow[t]{2}{*}{$2 \mathrm{KO}$} & $-] /[-$ & $2: 730$ \\
\hline & & & & & & $2: 695$ \\
\hline \multirow[t]{2}{*}{ D140C } & \multirow[t]{2}{*}{ DA } & \multirow[t]{2}{*}{ T/C Value Out of Ran } & \multirow[t]{2}{*}{ DEV1:N7:153/1 } & \multirow[t]{2}{*}{$2 \mathrm{~K} 3$} & $-] /[-$ & $2: 731$ \\
\hline & & & & & & $2: 696$ \\
\hline \multirow[t]{2}{*}{ D141A } & \multirow[t]{2}{*}{ DA } & \multirow[t]{2}{*}{ T/C Value Out of Ran } & \multirow[t]{2}{*}{ DEV1:N7:153/2 } & \multirow[t]{2}{*}{$2 \mathrm{~K} 6$} & $-] /$ & 2:734 \\
\hline & & & & & & $2: 698$ \\
\hline \multirow[t]{2}{*}{ D141B } & \multirow[t]{2}{*}{$\mathrm{DA}$} & \multirow[t]{2}{*}{$T / C$ Value Out of Ran } & \multirow[t]{2}{*}{ DEV1:N7:153/3 } & $2 \mathrm{Kg}$ & $-] /[-$ & $2: 735$ \\
\hline & & & & & & $2: 699$ \\
\hline D141C & $\mathrm{DA}$ & T/C Value Out of Ran & DEV1:N7:153/4 & $2 \mathrm{LO}$ & $-] /[-$ & 2:736 \\
\hline & & & & & & $2: 700$ \\
\hline D142A & $\mathrm{DA}$ & $\mathrm{T} / \mathrm{C}$ Value Out of Ran & DEV1:N7:153/5 & $2 \mathrm{~L} 3$ & $-] /[-$ & $2: 739$ \\
\hline & & & & & & $2: 702$ \\
\hline D142B & $\mathrm{DA}$ & T/C Value Out of Ran & DEV1:N7:153/6 & 2L6 & $-] /[-$ & $2: 740$ \\
\hline & & & & & & $2: 703$ \\
\hline PSH1 & $\mathrm{DA}$ & pressure switch alm & DEV1:N7:246/0 & $1 \mathrm{Co}$ & -() & $2: 114$ \\
\hline PSH2 & $\mathrm{DA}$ & pressure switch alm & DEV1:N7:246/1 & $1 C 1$ & -()$-$ & $2: 115$ \\
\hline $\mathrm{PSH} 3$ & $\mathrm{DA}$ & pressure switch alm & DEV1:N7:246/2 & $1 \mathrm{C} 2$ & -()$-$ & $2: 116$ \\
\hline PSH4 & $\mathrm{DA}$ & pressure switch alm & DEV1:N7:246/4 & $1 \mathrm{C3}$ & -()$-$ & $2: 117$ \\
\hline
\end{tabular}




\begin{tabular}{|c|c|c|c|c|c|c|}
\hline CV110A & $\mathrm{AO}$ & TY-110A PID_CV & DEV1:N7:154 & $2 \mathrm{M} 00$ & PID & 2:195 \\
\hline CVRD110A & Al & CV READBACK & DEV1:N7:154 & $2 \mathrm{M} 00$ & SCL & 2:195 \\
\hline CV110B & $\mathrm{AO}$ & TY-110B PID_CV & DEV1:N7:155 & 2M01 & PID & $2: 203$ \\
\hline CVRD110B & $\mathrm{Al}$ & CV READBACK & DEV1:N7:155 & $2 \mathrm{M} 01$ & SCL & $2: 203$ \\
\hline CV110C & $\mathrm{AO}$ & TY-110C PID_CV & DEV1:N7:156 & $2 \mathrm{MO} 2$ & PID & $2: 211$ \\
\hline CVRD110C & $\mathrm{Al}$ & CV READBAC̄K & DEV1:N7:156 & $2 \mathrm{M} 02$ & SCL & $2: 211$ \\
\hline CV111A & $\mathrm{AO}$ & TY-111A PID_cV & DEV1:N7:157 & $2 \mathrm{M03}$ & PID & $2: 219$ \\
\hline CVRD111A & $\mathrm{Al}$ & CV READBAC̄K & DEV1:N7:157 & $2 \mathrm{MO3}$ & SCL & $2: 219$ \\
\hline CV111B & $A O$ & TY-111B PID_CV & DEV1:N7:158 & $2 \mathrm{MO4}$ & PID & $2: 227$ \\
\hline CVRD111B & Al & CV READBACKK & DEV1:N7:158 & $2 \mathrm{M} 04$ & $\mathrm{SCL}$ & $2: 227$ \\
\hline CV111C & $\mathrm{AO}$ & TY-111C PID_CV & DEV1:N7:159 & $2 M 05$ & PID & $2: 236$ \\
\hline CVRD111C & $\mathrm{Al}$ & CV READBAC̄K & DEV1:N7:159 & $2 \mathrm{M} 05$ & $\mathrm{SCL}$ & $2: 236$ \\
\hline CV112A & $A O$ & TY-112A PID_CV & DEV1:N7:160 & $2 \mathrm{M} 06$ & PID & $2: 389$ \\
\hline CVRD112A & $\mathrm{Al}$ & CV READBAC KK & DEV1:N7:160 & $2 \mathrm{M} 06$ & $S C L$ & $2: 389$ \\
\hline CV112B & $A O$ & TY-112B PID_CV & DEV1:N7:161 & $2 \mathrm{M} 07$ & PID & $2: 397$ \\
\hline CVRD112B & Al & CV READBAC̄K & DEV1:N7:161 & $2 \mathrm{M} 07$ & SCL & $2: 397$ \\
\hline CV112C & $\mathrm{AO}$ & TY-112C PID_CV & DEV1:N7:162 & $2 \mathrm{M} 08$ & PID & $2: 405$ \\
\hline CVRD112C & Al & CV READBAC $\overrightarrow{\mathrm{CK}}$ & DEV1:N7:162 & $2 \mathrm{M08}$ & SCL & $2: 405$ \\
\hline CV113A & $\mathrm{AO}$ & TY-113A PID_CV & DEV1:N7:163 & $2 \mathrm{M} 09$ & PID & $2: 413$ \\
\hline CVRD113A & Al & CV READBAC K & DEV1:N7:163 & $2 \mathrm{M} 09$ & SCL & $2: 413$ \\
\hline CV113B & $\mathrm{AO}$ & TY-113B PID_CV & DEV1:N7:164 & $2 M 10$ & PID & $2: 421$ \\
\hline CVRD113B & Al & CV READBAC K & DEV1:N7:164 & $2 \mathrm{M} 10$ & $\mathrm{SCL}$ & $2: 421$ \\
\hline CV113c & $A O$ & TY-113C PID_CV & DEV1:N7:165 & $2 M 11$ & PID & $2: 430$ \\
\hline CVRD113C & Al & CV READBAC̄K & DEV1:N7:165 & $2 M 11$ & SCL. & $2: 430$ \\
\hline CV119 & $\mathrm{AO}$ & TY-119 PID_CV & DEV1:N7:166 & $2 \mathrm{M} 12$ & PID & $2: 561$ \\
\hline CVRD119 & $\mathrm{Al}$ & CV READBĀCK & DEV1:N7:166 & $2 \mathrm{M} 12$ & SCL & $2: 561$ \\
\hline CV120 & $\mathrm{AO}$ & TY-120 PID_CV & DEV1:N7:167 & $2 \mathrm{M} 13$ & PID & $2: 569$ \\
\hline CVRD120 & $\mathrm{Al}$ & CV READBACK & DEV1:N7:167 & $2 \mathrm{M} 13$ & $S C L$ & $2: 569$ \\
\hline CV121 & $\mathrm{AO}$ & TY-121 PID_CV & DEV1:N7:168 & $2 \mathrm{M} 14$ & PID & $2: 577$ \\
\hline CVRD121 & $\mathrm{Al}$ & CV READBĀCK & DEV1:N7:168 & $2 \mathrm{M} 14$ & $\mathrm{SCL}$ & $2: 577$ \\
\hline CV122 & $\mathrm{AO}$ & TY-122 PID_CV & DEV1:N7:169 & $2 \mathrm{M} 15$ & PID & $2: 585$ \\
\hline CVRD122 & $\mathrm{Al}$ & CV READBĀCK & DEV1:N7:169 & $2 \mathrm{M} 15$ & $\mathrm{SCL}$ & $2: 585$ \\
\hline CV123 & $\mathrm{AO}$ & TY-123 PID_CV & DEV1:N7:170 & $2 \mathrm{M} 16$ & PID & $2: 593$ \\
\hline CVRD123 & $\mathrm{Al}$ & CV READBĀCK & DEV1:N7:170 & $2 \mathrm{M} 16$ & $\mathrm{SCL}$ & $2: 593$ \\
\hline CV 124 & $A O$ & TY-124 PID_CV & DEV1:N7:171 & $2 \mathrm{M} 17$ & PID & $2: 601$ \\
\hline CVRD124 & $\mathrm{Al}$ & CV READBACCK & DEV1:N7:171 & $2 \mathrm{M} 17$ & SCL & $2: 601$ \\
\hline CV125 & $A O$ & TY-125 PID_CV & DEV1:N7:172 & $2 \mathrm{M} 18$ & PID & $2: 610$ \\
\hline CVRD125 & $\mathrm{Al}$ & CV READBACCK & DEV1:N7:172 & $2 \mathrm{M} 18$ & SCL. & $2: 610$ \\
\hline CV130 & AO & TY-130 PID_CV & DEV1:N7:173 & $2 \mathrm{M} 19$ & PID & $2: 749$ \\
\hline CVRD130 & Al & CV READBACCK & DEV1:N7:173 & $2 \mathrm{M} 19$ & $S C L$ & $2: 749$ \\
\hline CV131 & $\mathrm{AO}$ & TY-131 PID_CV & DEV1:N7:174 & $2 \mathrm{M} 20$ & PID & $2: 757$ \\
\hline CVRD131 & Al & CV READBĀCK & DEV1:N7:174 & $2 \mathrm{M} 20$ & SCL & $2: 757$ \\
\hline CV132 & $\mathrm{AO}$ & TY-132 PID_CV & DEV1:N7:175 & $2 M 21$ & PID & $2: 765$ \\
\hline CVRD132 & $\mathrm{Al}$ & CV READBACCK & DEV1:N7:175 & $2 M 21$ & SCL & $2: 765$ \\
\hline CV133 & $\mathrm{AO}$ & TY-133 PID_CV & DEV1:N7:176 & $2 \mathrm{M} 22$ & PID & $2: 773$ \\
\hline CVRD133 & $\mathrm{Al}$ & CV READBĀCK & DEV1:N7:176 & $2 \mathrm{M} 22$ & SCL & $2: 773$ \\
\hline CV134 & $A O$ & TY-134 PID_CV & DEV1:N7:177 & $2 \mathrm{M} 23$ & PID & $2: 781$ \\
\hline CVRD134 & Al & CV READBACCK & DEV1:N7:177 & $2 \mathrm{M} 23$ & $S C L$ & $2: 781$ \\
\hline CV140 & $A O$ & TY-140 PID_CV & DEV1:N7:178 & $2 \mathrm{M} 24$ & PID & $2: 789$ \\
\hline CVRD140 & $\mathrm{Al}$ & CV READBACCK & DEV1:N7:178 & $2 \mathrm{M} 24$ & SCL & $2: 78$ \\
\hline CV141 & $A O$ & TY-141 PID CV & DEV1:N7:179 & $2 M 25$ & PID & $2: 79$ \\
\hline
\end{tabular}


Tag Name Tag Type Description

CVRD141 AI CV READBACK

CV142

CVRD142
AO

Al

TY-142 PID CV

CV READBĀCK
PLC Device PLC 1/O Ladder Logic Rungs

DEV1:N7:179 2M2J $\quad$ SCL $\quad 2: 797$

DEV1:N7:180 2M26 PID $2: 806$

DEV1:N7:180 2M26 SCL $2: 806$

$\begin{array}{ll}\text { Address } & \text { Symbol } / \\ & \text { Instruction File Number:Rung Number } \\ \text { N7:182 } & \\ & \text { PH110A } \\ & \text {-CLR- } 2: 192 \\ & \text {-LES- } 2: 830 \\ & \text {-SCL- } 2: 195 \\ \text { N7:183 } & \text { PW110B } \\ & \text {-CLR- } 2: 200 \\ & \text {-LES- } 2: 833 \\ & \text {-SCL- } 2: 203 \\ \text { N7:184 } & \text { PH110C } \\ & \text {-CLR- } 2: 208 \\ & \text {-LES- } 2: 836 \\ & \text {-SCL- 2:211 } \\ & \text { PW111A } \\ \text { N7:185 } & \text {-CLR- } 2: 216 \\ & \text {-LES- } 2: 839 \\ \text {-SCL- } 2: 219\end{array}$


Processor and Data(OPS Unit 1)

October 23, 1996

Processor File: SODIUMIA.ACH

Addregs

Symbol /

Comment /

Instruction File Number:Rung Number

N7: 186

PH111B

-CLR- 2:224

-LES- $2: 842$

$-\mathrm{SCL}-\quad 2: 227$

HNF-SD-FF-CSWD-60Rev. 0

N7 $: 187$

PW111C

-CLR - 2:233

-LES- $2: 845$

$-\mathrm{SCL}-\quad 2: 236$

N7 $: 188$

PW112A

-CLR- 2:386

-LES- 2:848

-SCL- 2:389

N7 : 189

PW112B

-CLR - 2:394

-LES- 2:B51

-SCL- 2:397

N7 : 190

PW112C

-CLR- 2:402

-LES- 2:854

-SCL- 2:405

N7 : 191

PW113A

-CLR - 2:410

-LES- 2:857

$-S C L-2: 413$

N7 : 192

PW113B

-CLR- 2:418

-LES- 2:860

-SCL- 2:421

N7 : 193

PW113C

-CLR - 2:427

-LES - 2:863

-SCL- $2: 430$

N7 : 194

PH119

-CLR - 2:558

-LES- 2:866

-SCL- 2:561

N7: 195

PH120

-CLR- 2:566

-LES- 2:869

-SCL- 2:569

N7 : 196

PW121

- CLR- 2:574

-LES - 2:872

-SCL- 2:577 


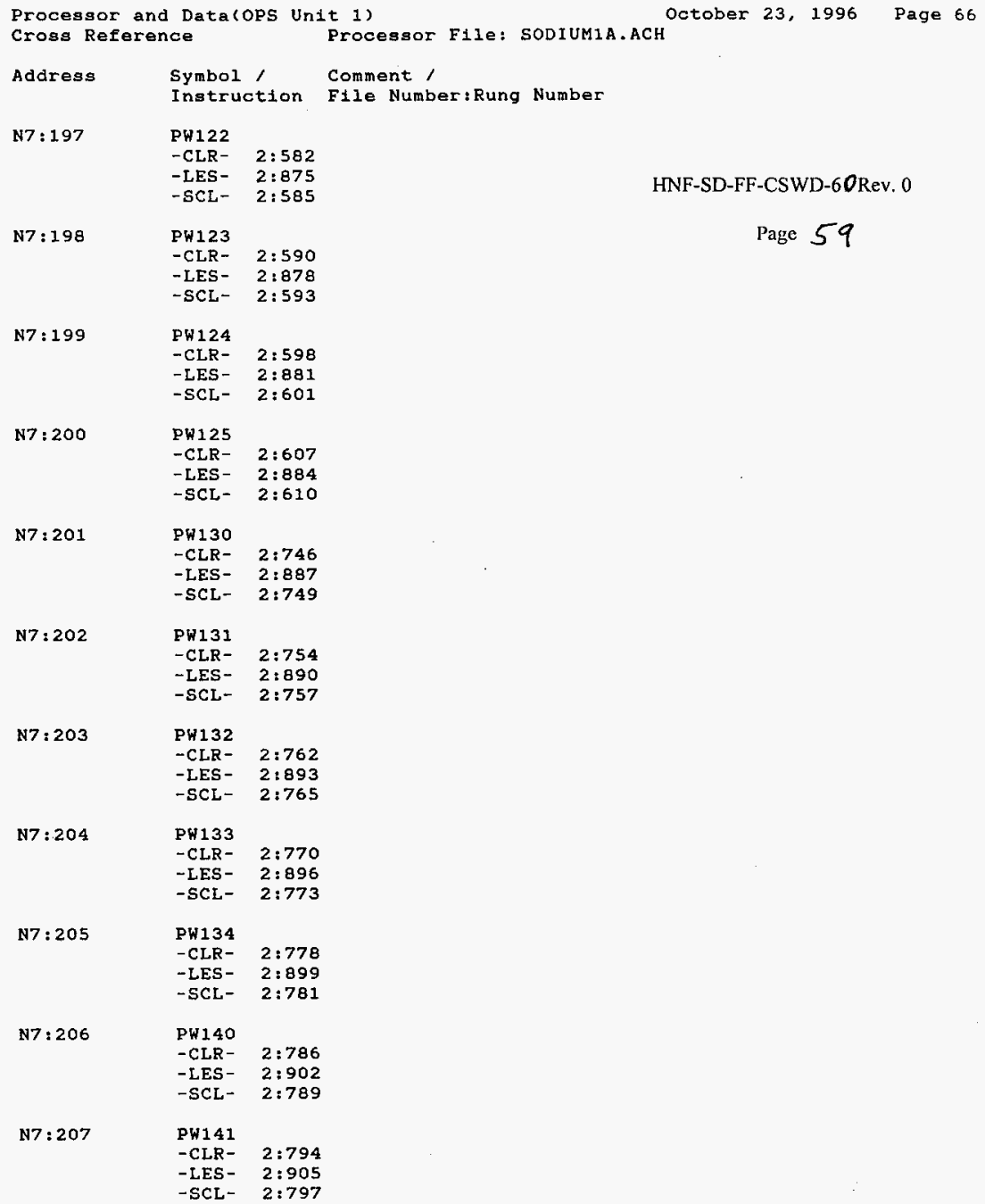


Processor and Data(OPS Unit 1 )

Cross Reference

$\begin{array}{ll}\text { Address } & \text { Symbol } \\ & \text { Instruction } \\ \text { N7:208 } & \text { PH142 } \\ & \text {-CLR- 2:803 } \\ & \text {-LES- 2:908 } \\ & \text {-SCL- 2:806 }\end{array}$

N7: $239 / 3$

$$
\begin{aligned}
& \text { ON_110A } \\
& -][-\quad 2: 821 \quad 2: 828 \quad 2: 830 \\
& -3 /[-\quad 2: 192 \\
& -()-2: 820
\end{aligned}
$$

N7 : $239 / 4$

$$
\begin{aligned}
& \text { ON_11OB } \\
& -][-2: 821 \quad 2: 831 \\
& -] /[-2: 200 \\
& -()-2: 820 .
\end{aligned}
$$

N7: $239 / 5$

ON_110C

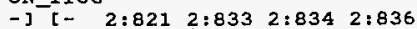

$-3 /[-\quad 2: 208$

-()$-2: 820$

N7 : 239/6

ON_111A

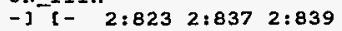

$-\mathrm{J} / \mathrm{C}-2: 216$

-()$-2: 822$

N7: $239 / 7$

ON_111B

$\begin{array}{lll}-] & 2: 823 \quad 2: 840 \quad 2: 842\end{array}$

$-] /[-2: 224$

-()$-2: 822$

N7 : 239/8

ON $111 \mathrm{C}$

$\begin{array}{lll}-] & 2: 823 \quad 2: 843 \quad 2: 845\end{array}$

$-] /[-2: 233$

-()$-2: 822$

N7 : $239 / 9$

\section{ON_112A}

$\begin{array}{llll}-1 & 2: 825 \quad 2: 846 \quad 2: 848\end{array}$

$-] /[-2: 386$

-()$-2: 824$

$N 7: 239 / 10$

ON_112B

- ] [- 2:825 2:849 2:851

$-] /[-2: 394$

-()$-2: 824$

N7: $239 / 11$

ON_ $112 \mathrm{C}$

$\begin{array}{llll}-] & \text { [ } & 2: 825 \quad 2: 852 \quad 2: 854\end{array}$

$-] /[-2: 402$

-()$-2: 824$

N7 : $239 / 12$

ON_113A

$\begin{array}{llll}-][-\quad 2: 827 \quad 2: 855 \quad 2: 857 & \end{array}$

$-] /[-2: 410$

-()$-2: 826$

N7: $239 / 13$ ON_113B

- ] [ $-2: 827 \quad 2: 858 \quad 2: 860$

$-] /[-\quad 2: 418$

-()$-2: 826$

$N 7: 239 / 14 \quad O N \_113 \mathrm{C}$

$\begin{array}{lllll}-3 & 2: 827 \quad 2: 861 \quad 2: 863\end{array}$

$-] /[-2: 427$

-()$-2: 826$
HNF-SD-FF-CSWD-61 Rev. 0

Page 60 


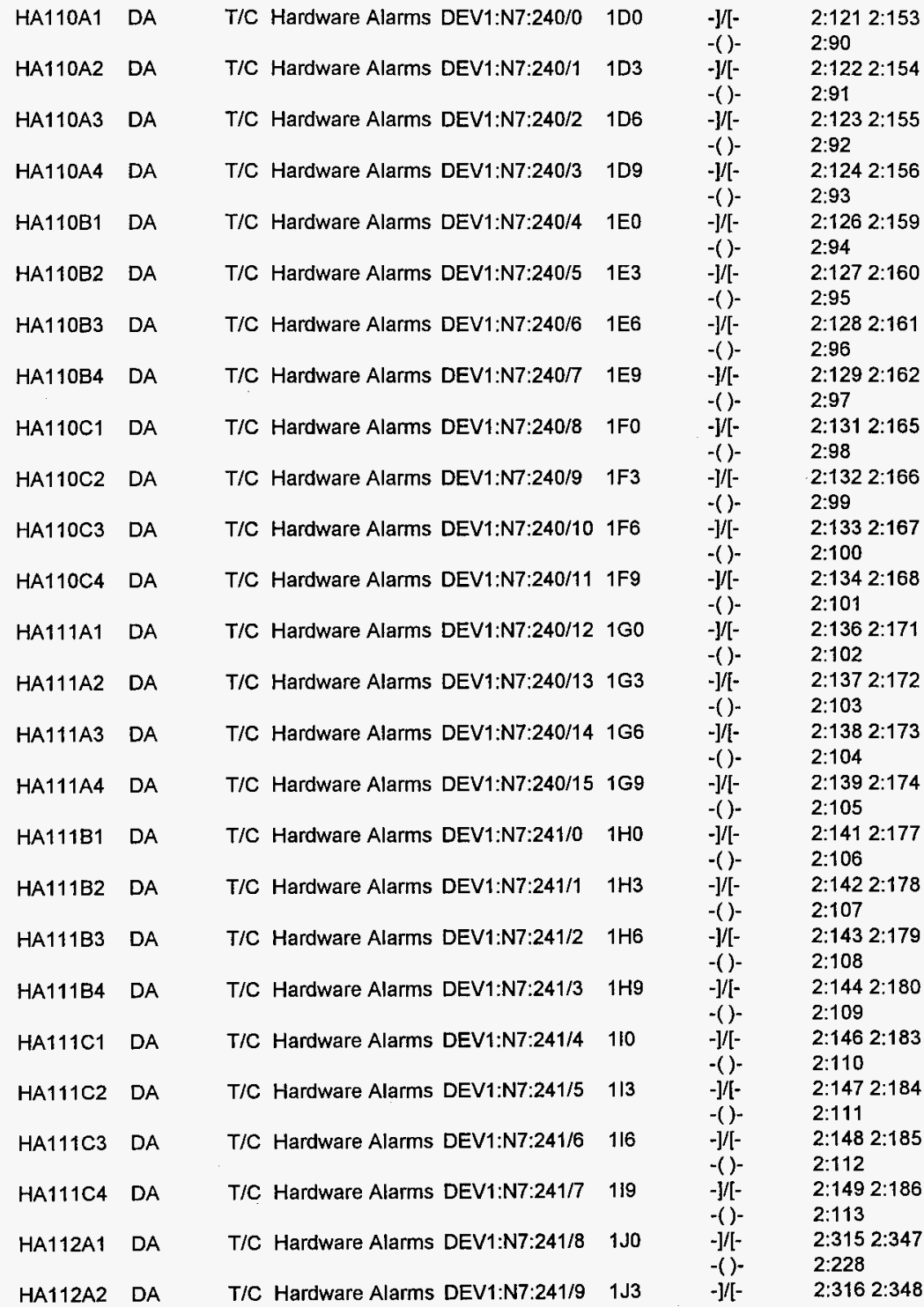




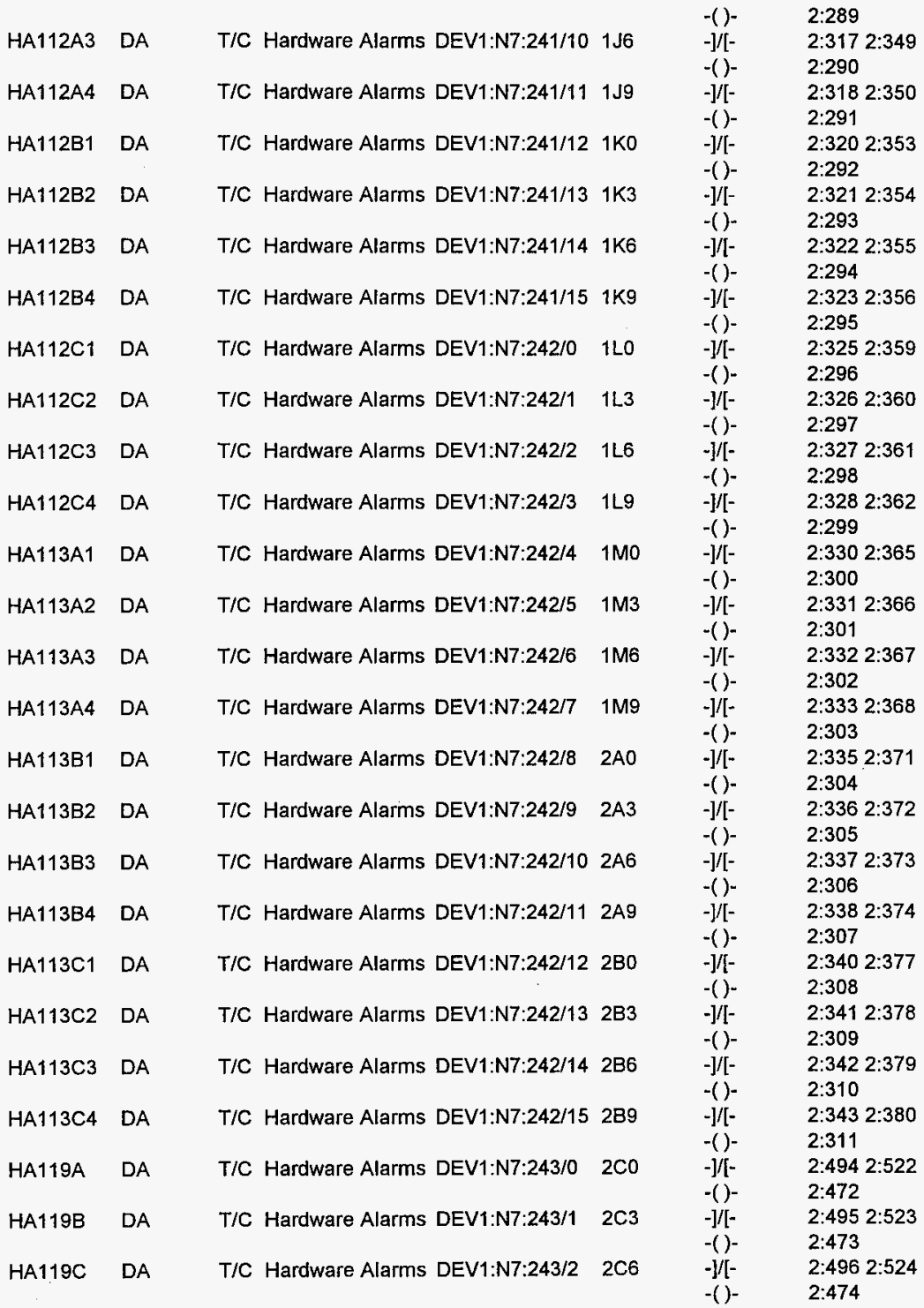




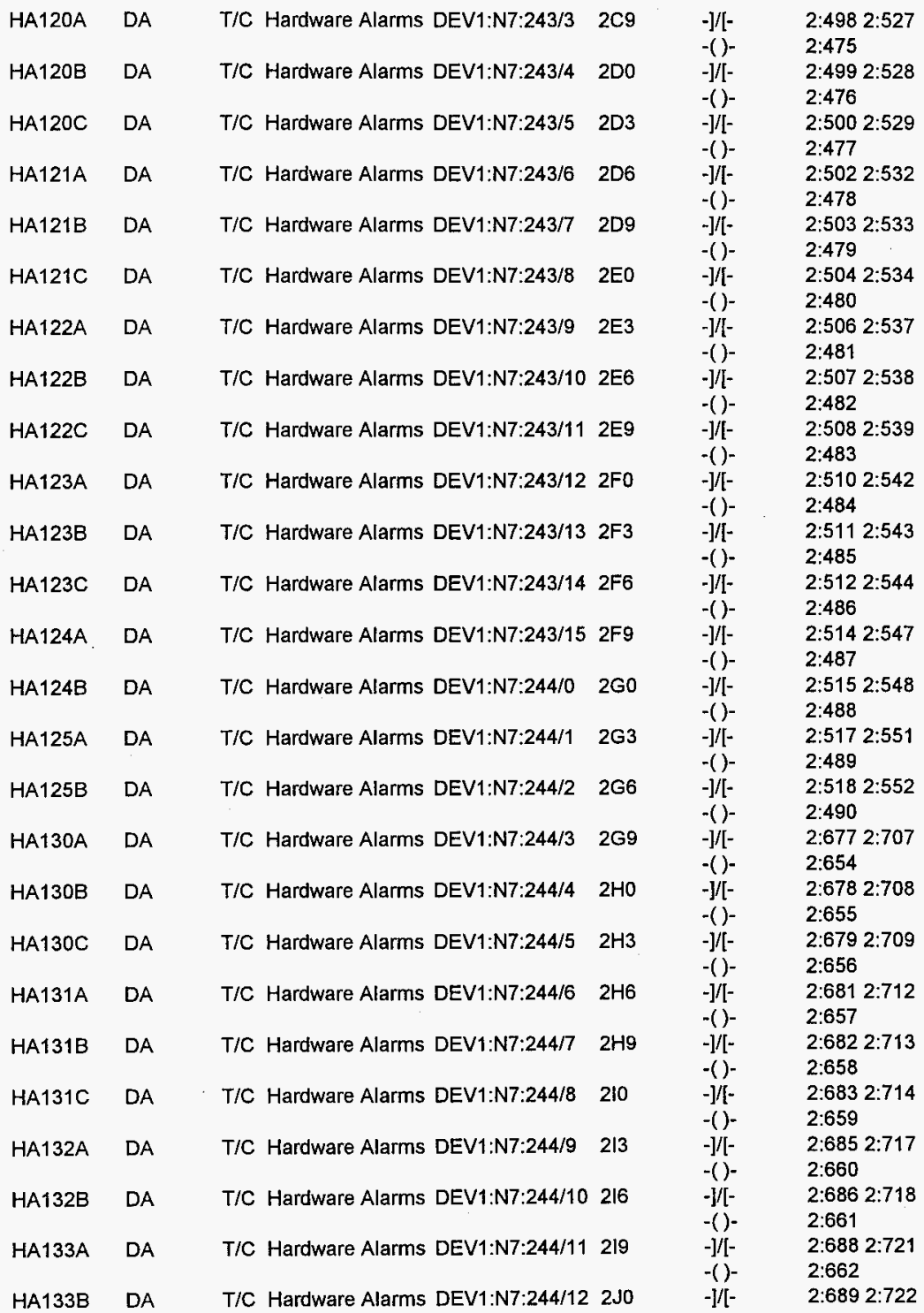




\begin{tabular}{|c|c|c|c|c|c|c|}
\hline \multirow{2}{*}{ HA134A } & \multirow[b]{2}{*}{$\mathrm{DA}$} & \multirow[b]{2}{*}{ T/C Hardware Alarms } & \multirow[b]{2}{*}{ DEV1:N7:244/13 } & \multirow[b]{2}{*}{$2 \mathrm{~J} 3$} & -()$-$ & $2: 663$ \\
\hline & & & & & $\begin{array}{l}-] /[- \\
-()-\end{array}$ & $\begin{array}{l}2: 6912: 725 \\
2: 664\end{array}$ \\
\hline HA134B & $\mathrm{DA}$ & T/C Hardware Alarms & DEV1:N7:244/14 & $2 \mathrm{~J} 6$ & $\begin{array}{l}-] /[- \\
-()-\end{array}$ & $\begin{array}{l}2: 6922: 726 \\
2: 665\end{array}$ \\
\hline HA140A & DA & T/C Hardware Alarms & DEV1:N7:244/15 & 2J9 & $\begin{array}{l}-] /[- \\
-()-\end{array}$ & $\begin{array}{l}2: 6942: 729 \\
2: 666\end{array}$ \\
\hline HA140B & DA & T/C Hardware Alarms & DEV1:N7:245/0 & $2 \mathrm{KO}$ & $\begin{array}{l}-] /[- \\
-()-\end{array}$ & $\begin{array}{l}2: 6952: 730 \\
2: 667\end{array}$ \\
\hline HA $140 \mathrm{C}$ & $\mathrm{DA}$ & T/C Hardware Alarms & DEV1:N7:245/1 & $2 \mathrm{~K} 3$ & $\begin{array}{l}-] /[- \\
-()-\end{array}$ & $\begin{array}{l}2: 6962: 731 \\
2: 668\end{array}$ \\
\hline HA141A & DA & T/C Hardware Alarms & DEV1:N7:245/2 & $2 \mathrm{~K} 6$ & $\begin{array}{l}-] /[- \\
-()-\end{array}$ & $\begin{array}{l}2: 6982: 734 \\
2: 669\end{array}$ \\
\hline HA141B & DA & T/C Hardware Alarms & DEV1:N7:245/3 & $2 \mathrm{~K} 9$ & $\begin{array}{l}-] /[- \\
-()-\end{array}$ & $\begin{array}{l}2: 6992: 735 \\
2: 670\end{array}$ \\
\hline HA141C & DA & T/C Hardware Alarms & DEV1:N7:245/4 & 2LO & $\begin{array}{l}-] /[- \\
-()-\end{array}$ & $\begin{array}{l}2: 7002: 736 \\
2: 671\end{array}$ \\
\hline HA142A & DA & T/C Hardware Alarms & DEV1:N7:245/5 & 2L3 & $\begin{array}{l}-] /[- \\
-()-\end{array}$ & $\begin{array}{l}2: 7022: 739 \\
2: 672\end{array}$ \\
\hline HA142B & DA & T/C Hardware Alarms & DEV1:N7:245/6 & 2L6 & $\begin{array}{l}-] /[- \\
-()-\end{array}$ & $\begin{array}{l}2: 7032: 740 \\
2: 673\end{array}$ \\
\hline PSH1 & DA & pressure switch alm & DEV1:N7:246/0 & $1 \mathrm{CO}$ & -()$-$ & $2: 114$ \\
\hline $\mathrm{PSH} 2$ & DA & pressure switch alm & DEV1:N7:246/1 & 1C1 & -()$-$ & $2: 115$ \\
\hline $\mathrm{PSH} 3$ & $\mathrm{DA}$ & pressure switch alm & DEV1:N7:246/2 & $1 \mathrm{C} 2$ & -()$-$ & $2: 116$ \\
\hline $\mathrm{PSH} 4$ & DA & pressure switch alm & DEV1:N7:246/3 & $1 \mathrm{C} 3$ & -()$-$ & $2: 117$ \\
\hline
\end{tabular}




\begin{tabular}{|c|c|c|c|c|c|}
\hline OUT110A1 & DO & TE TAKE-OUT & DEV1:N7:246:4 & $-] /[-$ & $2: 153$ \\
\hline OUT110A2 & DO & TE TAKE-OUT & DEV1:N7:246:5 & $-] /[-$ & $2: 154$ \\
\hline OUT110A3 & DO & TE TAKE-OUT & DEV1:N7:246:6 & $-] /[-$ & $2: 155$ \\
\hline OUT110A4 & DO & TE TAKE-OUT & DEV1:N7:246:7 & $-1 / \pi-$ & $2: 156$ \\
\hline OUT11081 & DO & TE TAKE-OUT & DEV1:N7:246:8 & $-] /[-$ & $2: 159$ \\
\hline OUT110B2 & DO & TE TAKE-OUT & DEV1:N7:246:9 & $-] /[-$ & $2: 160$ \\
\hline OUT110B3 & DO & TE TAKE-OUT & DEV1:N7:246:10 & $-] /[-$ & 2:161 \\
\hline OUT110B4 & DO & TE TAKE-OUT & DEV1:N7:246:11 & $-] /[-$ & $2: 162$ \\
\hline OUT110C1 & DO & TE TAKE-OUT & DEV1:N7:246:12 & $-] /[-$ & $2: 165$ \\
\hline OUT110C2 & DO & TE TAKE-OUT & DEV1:N7:246:13 & $-] /[-$ & $2: 166$ \\
\hline OUT110C3 & DO & TE TAKE-OUT & DEV1:N7:246:14 & $-] /[-$ & $2: 167$ \\
\hline OUT110C4 & DO & TE TAKE-OUT & DEV1:N7:246:15 & $-] /[-$ & $2: 168$ \\
\hline OUT111A1 & DO & TE TAKE-OUT & DEV1:N7:247:0 & $-] /[-$ & $2: 171$ \\
\hline OUT111A2 & DO & TE TAKE-OUT & DEV1:N7:247:1 & $-] /[-$ & $2: 172$ \\
\hline OUT111A3 & DO & TE TAKE-OUT & DEV1:N7:247:2 & $-] /[-$ & $2: 173$ \\
\hline OUT111A4 & DO & TE TAKE-OUT & DEV1:N7:247:3 & $-] /[-$ & $2: 174$ \\
\hline OUT111B1 & DO & TE TAKE-OUT & DEV1:N7:247:4 & $-] /[-$ & $2: 177$ \\
\hline OUT111B2 & DO & TE TAKE-OUT & DEV1:N7:247:5 & $-] /[-$ & $2: 178$ \\
\hline OUT111B3 & DO & TE TAKE-OUT & DEV1:N7:247:6 & $-] /[-$ & $2: 179$ \\
\hline OUT111B4 & DO & TE TAKE-OUT & DEV1:N7:247:7 & $-] /[-$ & $2: 180$ \\
\hline OUT111C1 & DO & TE TAKE-OUT & DEV1:N7:247:8 & $-] /[-$ & $2: 183$ \\
\hline OUT111C2 & DO & TE TAKE-OUT & DEV1:N7:247:9 & $-] /[-$ & $2: 184$ \\
\hline OUT111C3 & DO & TE TAKE-OUT & DEV1:N7:247:10 & $-] /[-$ & $2: 185$ \\
\hline OUT111C4 & DO & TE TAKE-OUT & DEV1:N7:247:11 & $-] /[-$ & \\
\hline OUT112A1 & DO & TE TAKE-OUT & DEV1:N7:247:12 & $-] /[-$ & $2: 347$ \\
\hline OUT112A2 & DO & TE TAKE-OUT & DEV1:N7:247:13 & $-] /[-$ & $2: 348$ \\
\hline OUT112A3 & DO & TE TAKE-OUT & DEV1:N7:247:14 & $-] /[-$ & $2: 349$ \\
\hline OUT112A4 & DO & TE TAKE-OUT & DEV1:N7:247:15 & $-] /[-$ & $2: 350$ \\
\hline OUT112B1 & DO & TE TAKE-OUT & DEV1:N7:248:0 & $-] /[-$ & $2: 353$ \\
\hline OUT112B2 & DO & TE TAKE-OUT & DEV1:N7:248:1 & $-] /[-$ & $2: 354$ \\
\hline OUT112B3 & DO & TE TAKE-OUT & DEV1:N7:248:2 & $-] /[-$ & $2: 355$ \\
\hline OUT112B4 & DO & TE TAKE-OUT & DEV1:N7:248:3 & $-] /[-$ & $2: 356$ \\
\hline OUT112C1 & DO & TE TAKE-OUT & DEV1:N7:248:4 & $-] /[-$ & $2: 359$ \\
\hline OUT112C2 & DO & TE TAKE-OUT & DEV1:N7:248:5 & $-] /[-$ & $2: 360$ \\
\hline OUT112C3 & DO & TE TAKE-OUT & DEV1:N7:248:6 & $-] /[-$ & $2: 361$ \\
\hline OUT112C4 & DO & TE TAKE-OUT & DEV1:N7:248:7 & $-] /[-$ & $2: 362$ \\
\hline OUT113A1 & DO & TE TAKE-OUT & DEV1:N7:248:8 & $-] /[-$ & $2: 365$ \\
\hline OUT113A2 & DO & TE TAKE-OUT & DEV1:N7:248:9 & $-] /[-$ & $2: 366$ \\
\hline OUT113A3 & DO & TE TAKE-OUT & DEV1:N7:248:10 & $-] /[-$ & $2: 367$ \\
\hline OUT113A4 & DO & TE TAKE-OUT & DEV1:N7:248:11 & $-] /[-$ & $2: 368$ \\
\hline OUT113B1 & DO & TE TAKE-OUT & DEV1:N7:248:12 & $-] /[-$ & $2: 371$ \\
\hline OUT113B2 & DO & TE TAKE-OUT & DEV1:N7:248:1.3 & $-] /[-$ & $2: 372$ \\
\hline OUT113B3 & DO & TE TAKE-OUT & DEV1:N7:248:14 & $-] /[-$ & $2: 373$ \\
\hline OUT11384 & DO & TE TAKE-OUT & DEV1:N7:248:15 & $-] / 1-$ & $2: 374$ \\
\hline OUT113C1 & DO & TE TAKE-OUT & DEV1:N7:249:0 & $-] /[-$ & $2: 377$ \\
\hline OUT113C2 & DO & TE TAKE-OUT & DEV1:N7:249:1 & $-] /[-$ & $2: 378$ \\
\hline OUT113C3 & DO & TE TAKE-OUT & DEV1:N7:249:2 & $-] /[-$ & $2: 379$ \\
\hline OUT113C4 & DO & TE TAKE-OUT & DEV1:N7:249:3 & $-] /[-$ & $2: 186$ \\
\hline OUT119A & DO & TE TAKE-OUT & DEV1:N7:249:4 & $-] /[-$ & $2: 522$ \\
\hline OUT119B & DO & TE TAKE-OUT & DEV1:N7:249:5 & $-] /[-$ & $2: 523$ \\
\hline OUT119C & DO & TE TAKE-OUT & DEV1:N7:249:6 & $-] /[-$ & $2: 524$ \\
\hline
\end{tabular}




\begin{tabular}{|c|c|c|c|c|c|}
\hline Tag Name & Tag type & Description & PLC Device & \multicolumn{2}{|c|}{ Ladder Logic Rungs } \\
\hline OUT120A & DO & TE TAKE-OUT & DEV1:N7:249:7 & $-] /[-$ & $2: 527$ \\
\hline OUT120B & DO & TE TAKE-OUT & DEV1:N7:249:8 & $-1 /[-$ & $2: 528$ \\
\hline OUT120C & DO & TE TAKE-OUT & DEV1:N7:249:9 & $-] /[-$ & $2: 529$ \\
\hline OUT121A & DO & TE TAKE-OUT & DEV1:N7:249:10 & $-] /[-$ & $2: 532$ \\
\hline OUT121B & DO & TE TAKE-OUT & DEV1:N7:249:11 & $-] /[-$ & $2: 533$ \\
\hline OUT121C & DO & TE TAKE-OUT & DEV1:N7:249:12 & $-] /[-$ & $2: 534$ \\
\hline OUT122A & DO & TE TAKE-OUT & DEV1:N7:249:13 & $-1 /[-$ & $2: 537$ \\
\hline OUT122B & DO & TE TAKE-OUT & DEV1:N7:249:14 & $-j /[-$ & $2: 538$ \\
\hline OUT122C & DO & TE TAKE-OUT & DEV1:N7:249:14 & $-1 /[-$ & $2: 539$ \\
\hline OUT123A & DO & TE TAKE-OUT & DEV1:N7:250:0 & $-] /[-$ & $2: 542$ \\
\hline OUT1238 & DO & TE TAKE-OUT & DEV1:N7:250:1 & $-] /[-$ & $2: 543$ \\
\hline OUT123C & DO & TE TAKE-OUT & DEV1:N7:250:2 & $-1 /[-$ & $2 ; 544$ \\
\hline OUT124A & DO & TE TAKE-OUT & DEV1:N7:250:3 & $-] /[-$ & $2: 547$ \\
\hline OUT124B & DO & TE TAKE-OUT & DEV1:N7:250:4 & $-] /[-$ & $2: 548$ \\
\hline OUT125A & DO & TE TAKE-OUT & DEV1:N7:250:5 & $-] /[-$ & $2: 551$ \\
\hline OUT125B & DO & TE TAKE-OUT & DEV1:N7:250:6 & $-] /[-$ & $2: 552$ \\
\hline OUT130A & DO & TE TAKE-OUT & DEV1:N7:250:7 & $-] /[-$ & $2: 707$ \\
\hline OUT130B & DO & TE TAKE-OUT & DEV1:N7:250:8 & $-] /[-$ & $2: 708$ \\
\hline OUT130C & DO & TE TAKE-OUT & DEV1:N7:250:9 & $-] /[-$ & \\
\hline OUT131A & DO & TE TAKE-OUT & DEV1:N7:250:10 & $-] /[-$ & $2: 712$ \\
\hline OUT131B & DO & TE TAKE-OUT & DEV1:N7:250:11 & $-] /[-$ & $2: 713$ \\
\hline OUT131C & DO & TE TAKE-OUT & DEV1:N7:250:12 & $-] /[-$ & $2: 714$ \\
\hline OUT132A & DO & TE TAKE-OUT & DEV1:N7:250:13 & $-] /[-$ & $2: 717$ \\
\hline OUT132B & DO & TE TAKE-OUT & DEV1:N7:250:14 & $-] /[-$ & $2: 718$ \\
\hline OUT133A & DO & TE TAKE-OUT & DEV1:N7:250:15 & $-] /[-$ & $2: 721$ \\
\hline OUT133B & DO & TE TAKE-OUT & DEV1:N7:251:0 & $-] /[-$ & $2: 722$ \\
\hline OUT134A & DO & TE TAKE-OUT & DEV1:N7:251:1 & $-] /[-$ & $2: 725$ \\
\hline OUT1348 & DO & TE TAKE-OUT & DEV1:N7:251:2 & $-] /[-$ & $2: 726$ \\
\hline OUT140A & DO & TE TAKE-OUT & DEV1:N7:251:3 & $-] /[-$ & $2: 729$ \\
\hline OUT140B & DO & TE TAKE-OUT & DEV1:N7:251:4 & $-] /[-$ & $2: 730$ \\
\hline OUT140C & DO & TE TAKE-OUT & DEV1:N7:251:5 & $-] /[-$ & 2:709 2:731 \\
\hline OUT141A & DO & TE TAKE-OUT & DEV1:N7:251:6 & $-] /[-$ & $2: 734$ \\
\hline OUT141B & DO & TE TAKE-OUT & DEV1:N7:251:7 & $-] /[-$ & $2: 735$ \\
\hline OUT141C & DO & TE TAKE-OUT & DEV1:N7:251:8 & $-] /[-$ & $2: 736$ \\
\hline OUT142A & DO & TE TAKE-OUT & DEV1:N7:251:9 & $-] /[-$ & $2: 739$ \\
\hline OUT142B & DO & TE TAKE-OUT & DEV1:N7:251:10 & $-] /[-$ & $2: 740$ \\
\hline
\end{tabular}




\begin{tabular}{|c|c|c|c|c|c|c|}
\hline Address & $\begin{array}{l}\text { Symbol } \\
\text { Ingtru }\end{array}$ & 'tion & $\begin{array}{l}\text { Commer } \\
\text { File }\end{array}$ & $\begin{array}{l}\text { nt } / \\
\text { Number }\end{array}$ & & Number \\
\hline N9: $20 / 15$ & -()$-$ & $2: 390$ & & & & \\
\hline N10:89 & $\begin{array}{l}\text { DEV I AT } \\
\text {-ADD- }\end{array}$ & $\begin{array}{l}\text { ION } \\
2: 120 \\
2: 334 \\
2: 680 \\
2: 120 \\
2: 334 \\
2: 680\end{array}$ & $\begin{array}{l}2: 125 \\
2: 339 \\
2: 684 \\
2: 125 \\
2: 339 \\
2: 684\end{array}$ & $\begin{array}{l}2: 130 \\
2: 493 \\
2: 687 \\
2: 130 \\
2: 493 \\
2: 687\end{array}$ & $\begin{array}{l}2: 135 \\
2: 497 \\
2: 690 \\
2: 135 \\
2: 497 \\
2: 690\end{array}$ & $\begin{array}{l}2: 140 \\
2: 501 \\
2: 693 \\
2: 140 \\
2: 501 \\
2: 693\end{array}$ \\
\hline N10:91 & $\begin{array}{l}\text { H11OA } \\
\text {-ADD- } \\
\text {-GRT- }\end{array}$ & $\begin{array}{l}2: 120 \\
2: 121\end{array}$ & $2: 122$ & $2: 123$ & $2: 124$ & \\
\hline N10: 92 & $\begin{array}{l}\text { H11OB } \\
\text {-ADD- } \\
\text {-GRT- }\end{array}$ & $\begin{array}{l}2: 125 \\
2: 126\end{array}$ & $2: 127$ & $2: 128$ & $2: 129$ & \\
\hline $\mathrm{N} 10: 93$ & $\begin{array}{l}\mathrm{H} 110 \mathrm{OC} \\
-A D D- \\
\text {-GRT- }\end{array}$ & $\begin{array}{l}2: 130 \\
2: 131\end{array}$ & $2: 132$ & $2: 133$ & $2: 134$ & \\
\hline N10:94 & $\begin{array}{l}\mathrm{H} 111 \mathrm{~A} \\
-\mathrm{ADD}- \\
-\mathrm{GRT}-\end{array}$ & $\begin{array}{l}2: 135 \\
2: 136\end{array}$ & $2: 137$ & $2: 138$ & $2: 139$ & \\
\hline N10:95 & $\begin{array}{l}\text { H111B } \\
\text {-ADD- } \\
\text {-GRT- }\end{array}$ & $\begin{array}{l}2: 140 \\
2: 141\end{array}$ & $2: 142$ & $2: 143$ & $2: 144$ & \\
\hline$N 10: 96$ & $\begin{array}{l}\text { H111C } \\
\text {-ADD- } \\
\text {-GRT- }\end{array}$ & $\begin{array}{l}2: 145 \\
2: 146\end{array}$ & $2: 147$ & $2: 148$ & $2: 149$ & \\
\hline N10:97 & $\begin{array}{l}\text { H112A } \\
\text { - ADD - } \\
\text {-GRT- }\end{array}$ & $\begin{array}{l}2: 314 \\
2: 315\end{array}$ & $2: 316$ & $2: 317$ & $2: 318$ & $2: 323$ \\
\hline$N 10: 98$ & $\begin{array}{l}\text { H112B } \\
\text { - ADD- } \\
\text {-GRT- }\end{array}$ & $\begin{array}{l}2: 319 \\
2: 320\end{array}$ & $2: 321$ & $2: 322$ & & \\
\hline N10:99 & $\begin{array}{l}H 112 \mathrm{C} \\
-A D D- \\
\text {-GRT- }\end{array}$ & $\begin{array}{l}2: 324 \\
2: 325\end{array}$ & $2: 326$ & $2: 327$ & $2: 328$ & \\
\hline $110: 100$ & $\begin{array}{l}\text { H113A } \\
\text {-ADD- } \\
\text {-GRT- }\end{array}$ & $\begin{array}{l}2: 329 \\
2: 330\end{array}$ & $2: 331$ & $2: 332$ & $2: 333$ & \\
\hline N10:101 & $\begin{array}{l}\text { HI13B } \\
-A D D- \\
\text { - GRT- }\end{array}$ & $\begin{array}{l}2: 334 \\
2: 335\end{array}$ & $2: 336$ & $2: 337$ & $2: 338$ & \\
\hline$N 10: 102$ & $\begin{array}{l}H 113 C \\
\text { - ADD- } \\
\text {-GRT- }\end{array}$ & $\begin{array}{l}2: 339 \\
2: 340\end{array}$ & $2: 341$ & $2: 342$ & $2: 343$ & \\
\hline
\end{tabular}

HNF-SD-FF-CSWD-60Rev. 0 


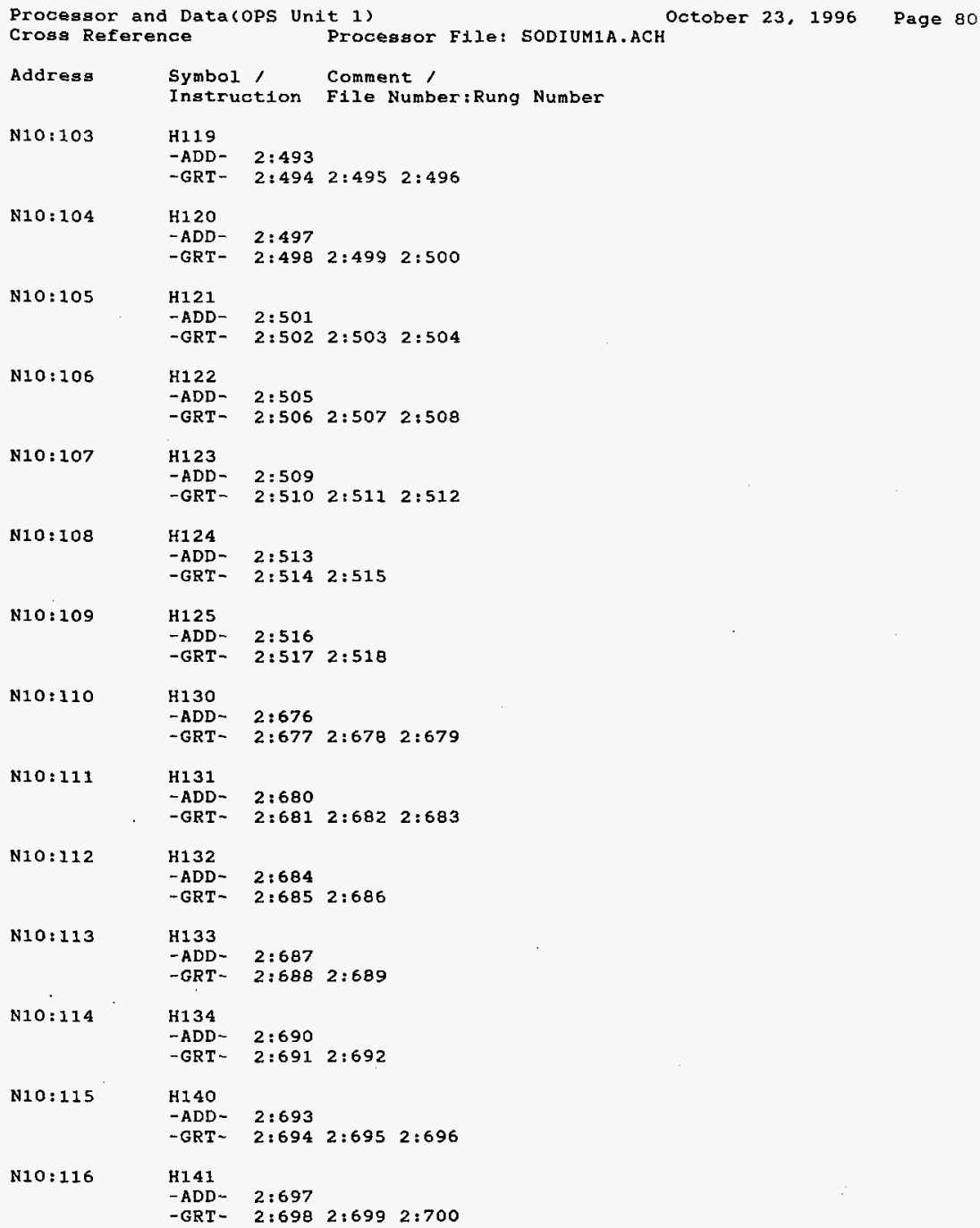




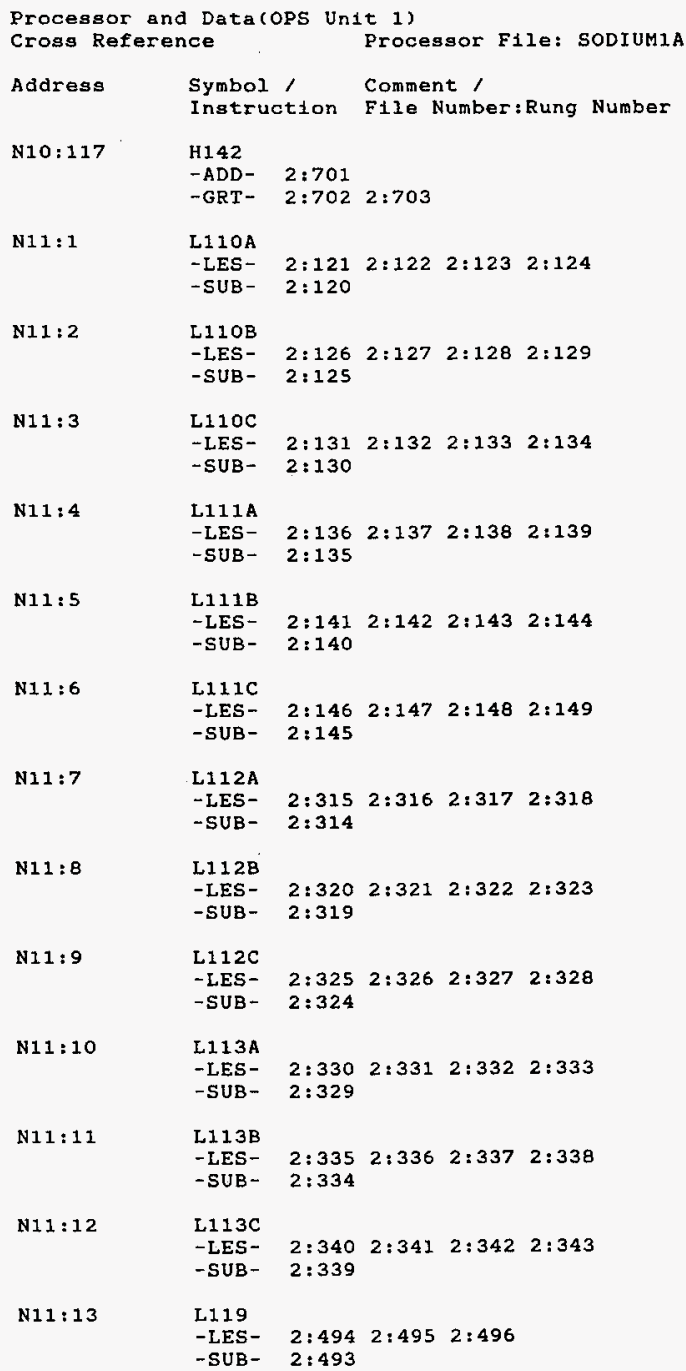

HNF-SD-FF-CSWD-60Rev. 0 


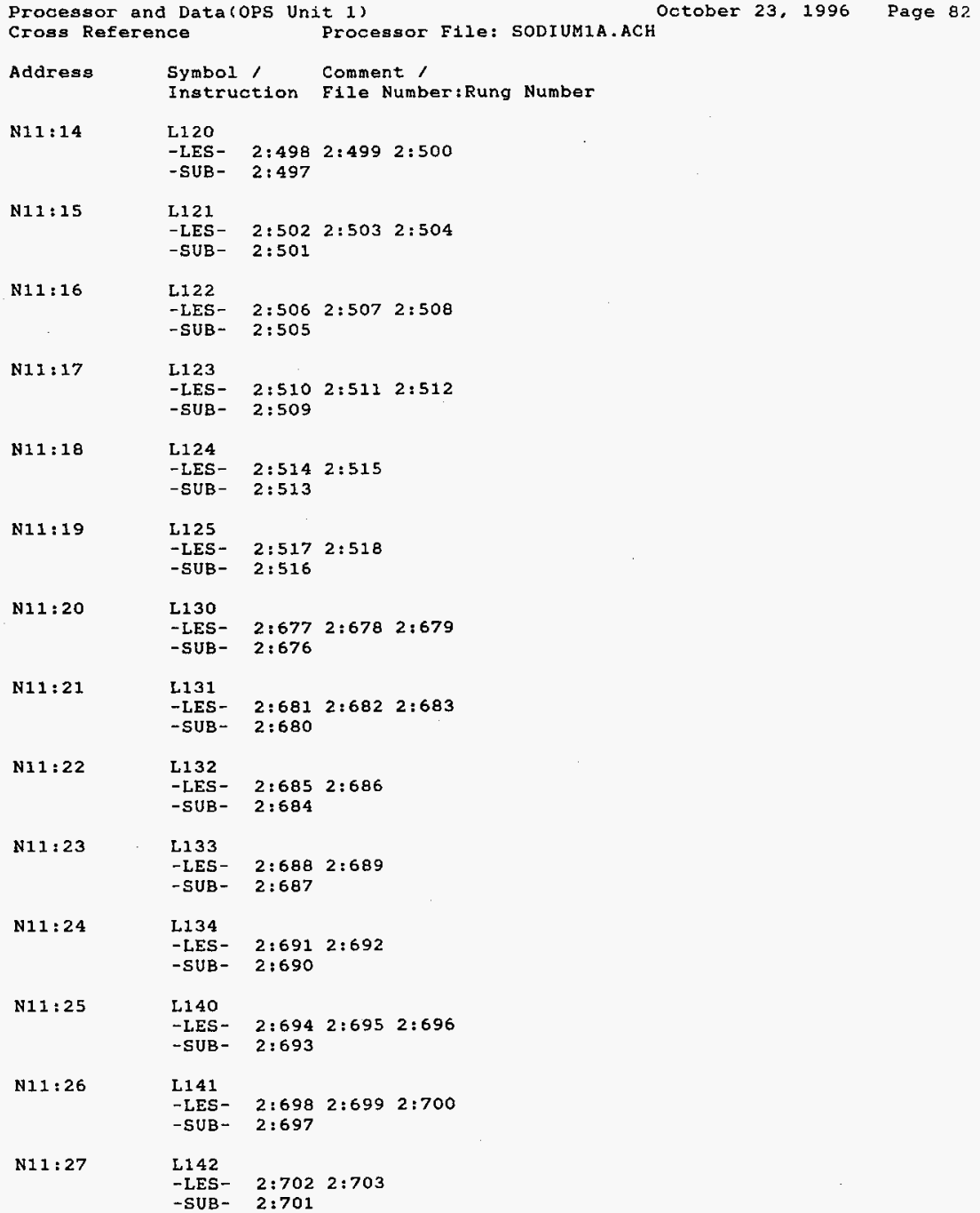


Processor and Data(ops Unit 1)

Cross Reference

Address

N12:0

$N 12: 0 / 1$

Symbol Instruction

Comment /

File Number:Rung Number

\section{PID110A}

-MOV- $3: 27$

-PID- 2:195

(1):

N12:1

$$
-()-2: 193
$$

N12:2

-CLR- $3: 54$

PIDSP110A

-MOV- 2:194

N12:11

-MOV- 2:190 2:190 2:190

-SUB- 2:190

N12:30

PID110B

-MOV- $3: 28$

-PID- $2: 203$

N12:30/1

-()$-2: 201$

N12:31

-CLR- $\quad 3: 55$

N12:32

PIDSP1108

-MOV- 2:202

N12:41

-NOV- 2:198 2:198 2:198

-SUB- 2:198

N12:60

PID110C

-MOV- 3:29

-PID- 2:211

$N 12: 60 / 1$

$$
-(1)-2: 209
$$

N1 2:61

$$
\text { - CLR- } 3: 56
$$

N12:62

PIDSP110C

MOV- $2: 210$

N12:71

-MOV- 2:206 2:206 2:206

-SUB- 2:206

N12:90

PID111A

- MOV- $3: 30$

-PID- 2:219

N12:90/1
HNF-SD-FF-CSWD-60 Rev. 0 


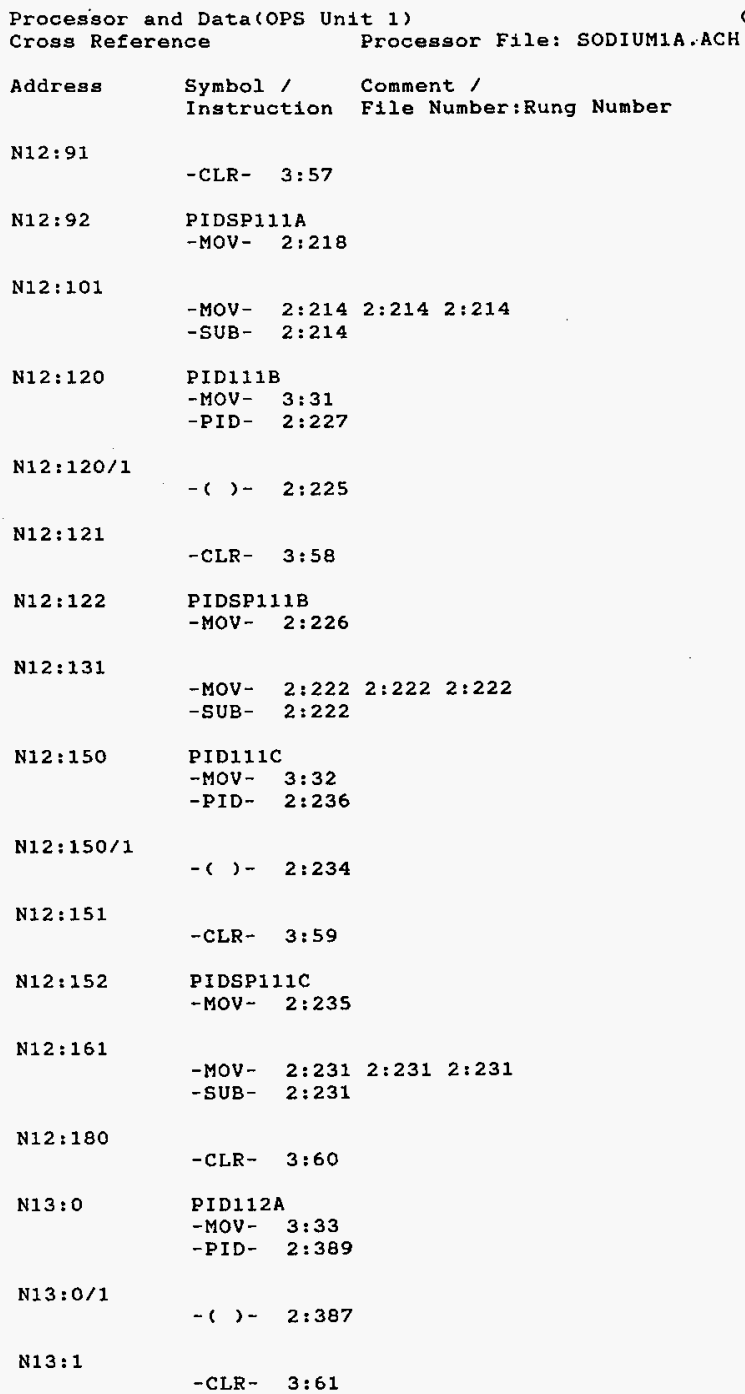

N13:1

$$
\text { -CLR- 3:61 }
$$




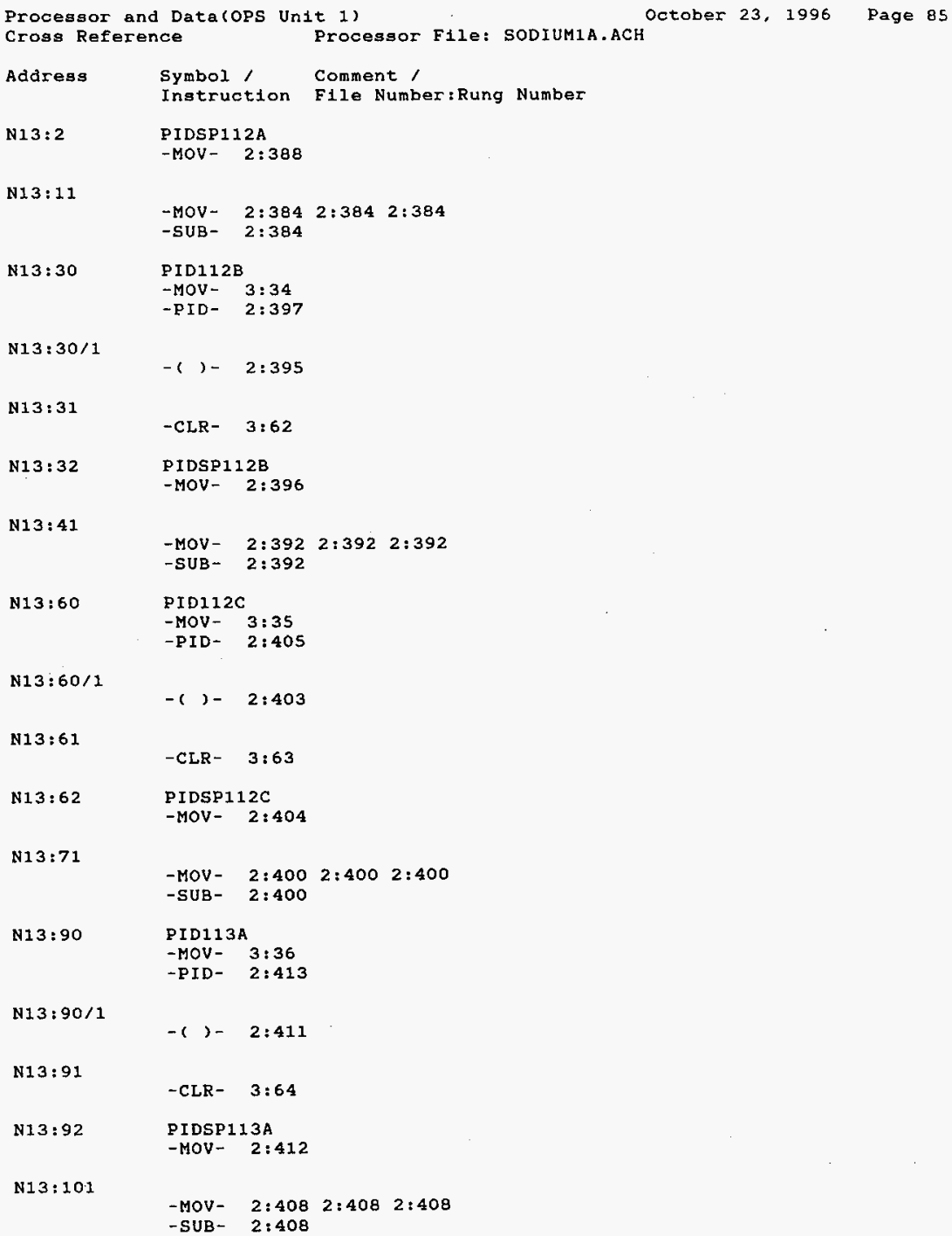




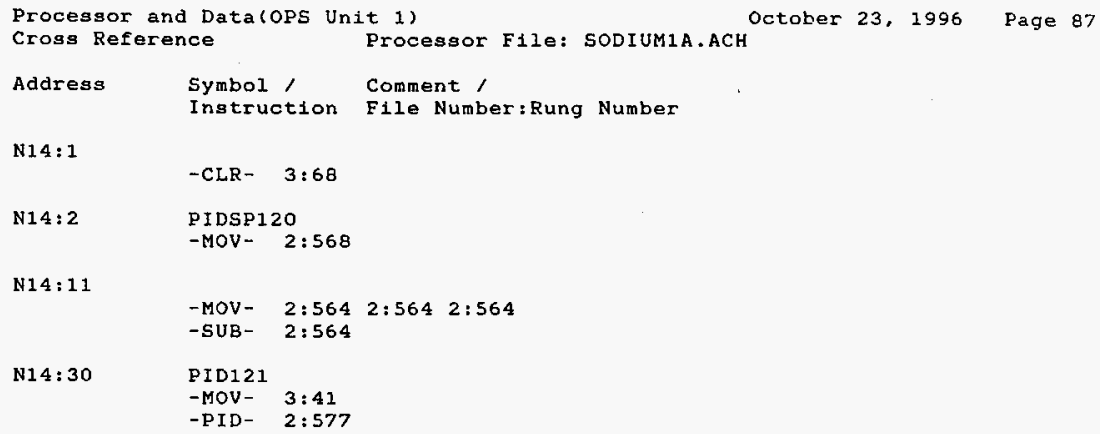

N14: 1

-CLR- $3: 68$

N14: 2

PIDSP120

-MOV- 2:568

N14:11

-MOV- 2:564 2:564 2:564

-SUB- 2:564

N14:30

PID121

-MOV- 3:41

-PID- 2:577

N14:30/1

-()$-2: 575$

N14:31

-CLR- $3: 69$

N14:32

PIDSP121

-MOV- 2:576

N14:41

-MOV- 2:572 2:572 2:572

-SUB- $2: 572$

N14:60

PID122

-MOV- 3:42

-PID- $2: 585$

N14:60/1

-()$-2: 583$

N14:61

-CLR- $3: 70$

N14:62

PIDSP122

-MOV- 2:584

N14:71

-MOV- 2:580 2:580 2:580

-SUB- $2: 580$

N14: 90

PID123

-MOV - $3: 43$

-PID- 2:593

N14:90/1

-()$-2: 591$

N1 4: 91

-CLR- 3:71

N14: 92

PIDSP123

-MOV- 2:592

HNF-SD-FF-CSWD-6DRev. 0

Page 74 


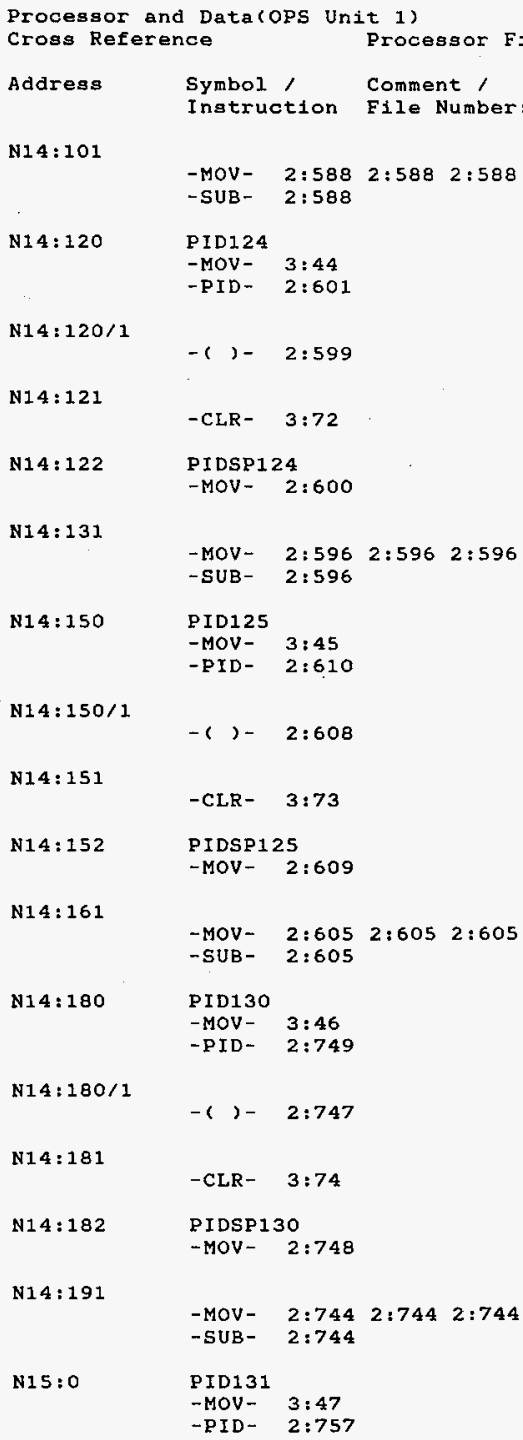

N14: 161

MOV- 2:605 2:605 2:605

N14:180

PID130

-MOV- $3: 46$

-PID- 2:749

N14: $180 / 1$

-()$-2: 747$

N14:181

-CLR- $\quad 3: 74$

N14: 182

PIDSP 130

-MOV- $2: 748$

N14: 191

-MOV- $2: 744 \quad 2: 744 \quad 2: 744$

-SUB- $2: 744$

N15:0

PID131

-MOV- $3: 47$

-PID- 2:757 


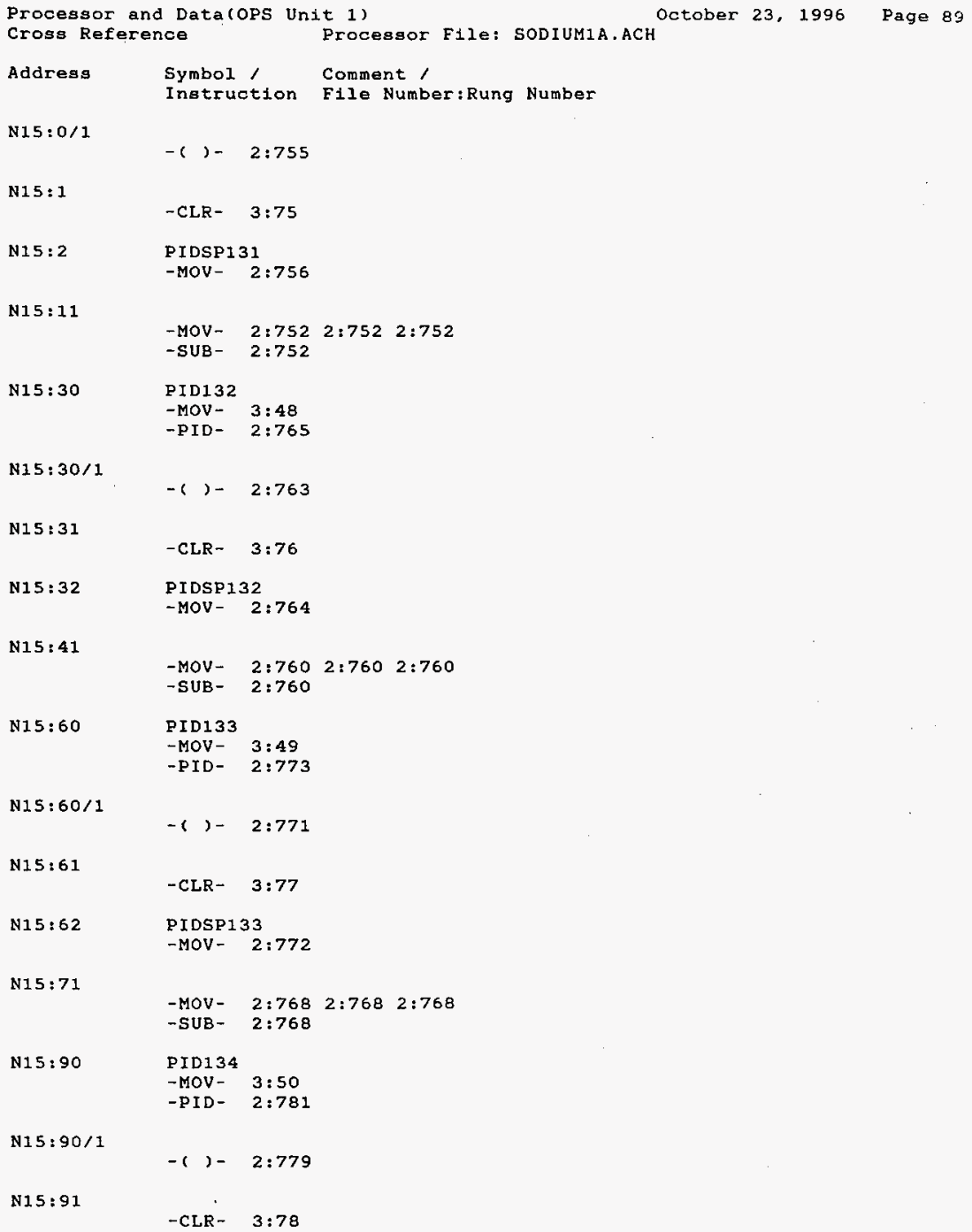

N1 $5: 90 / 1$

$-(1)-2: 779$

N1 $5: 91$

-CLR- $3: 78$

HNF-SD-FF-CSWD-60 Rev. 0 


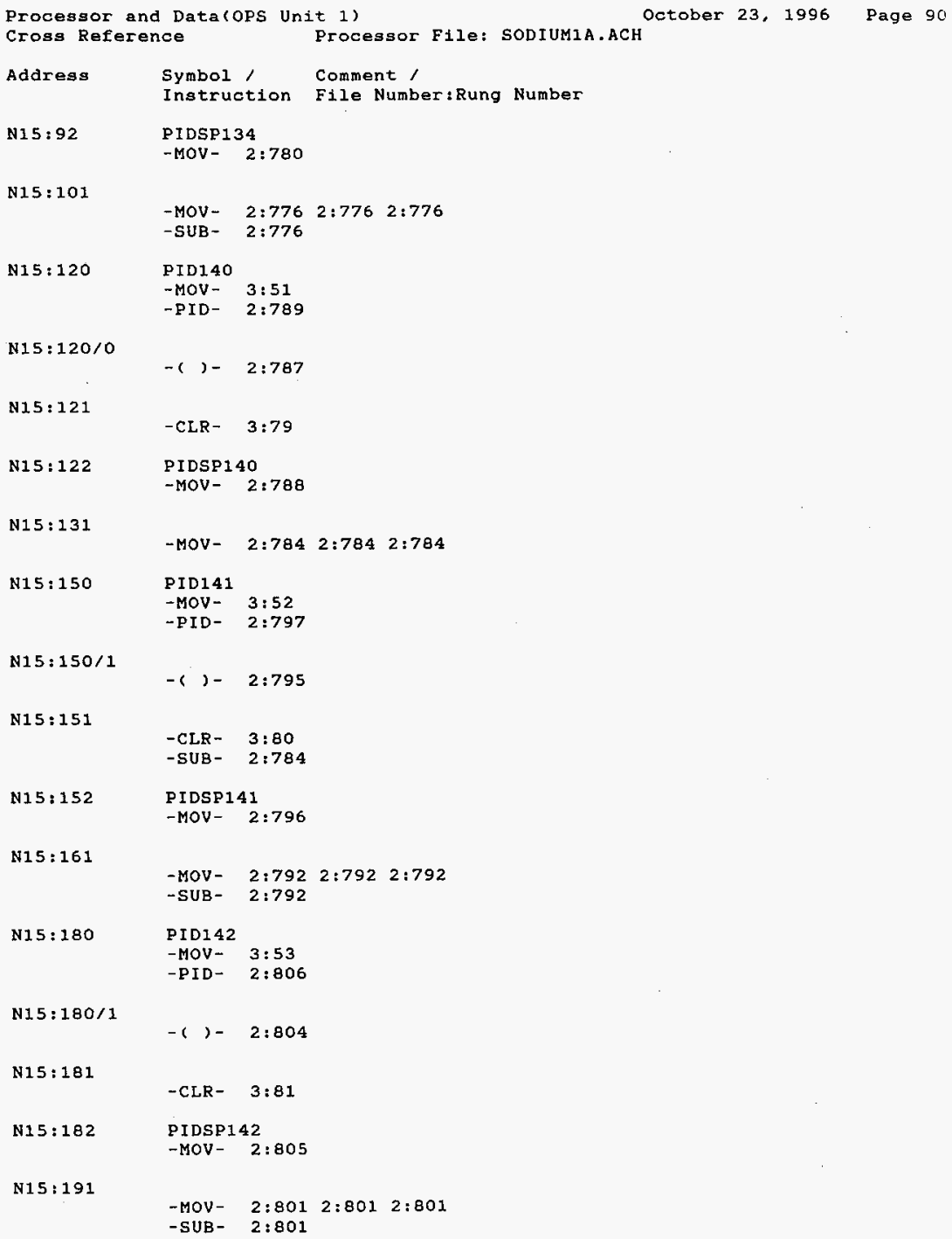

HNF-SD-FF-CSWD-6 ORev. 0 


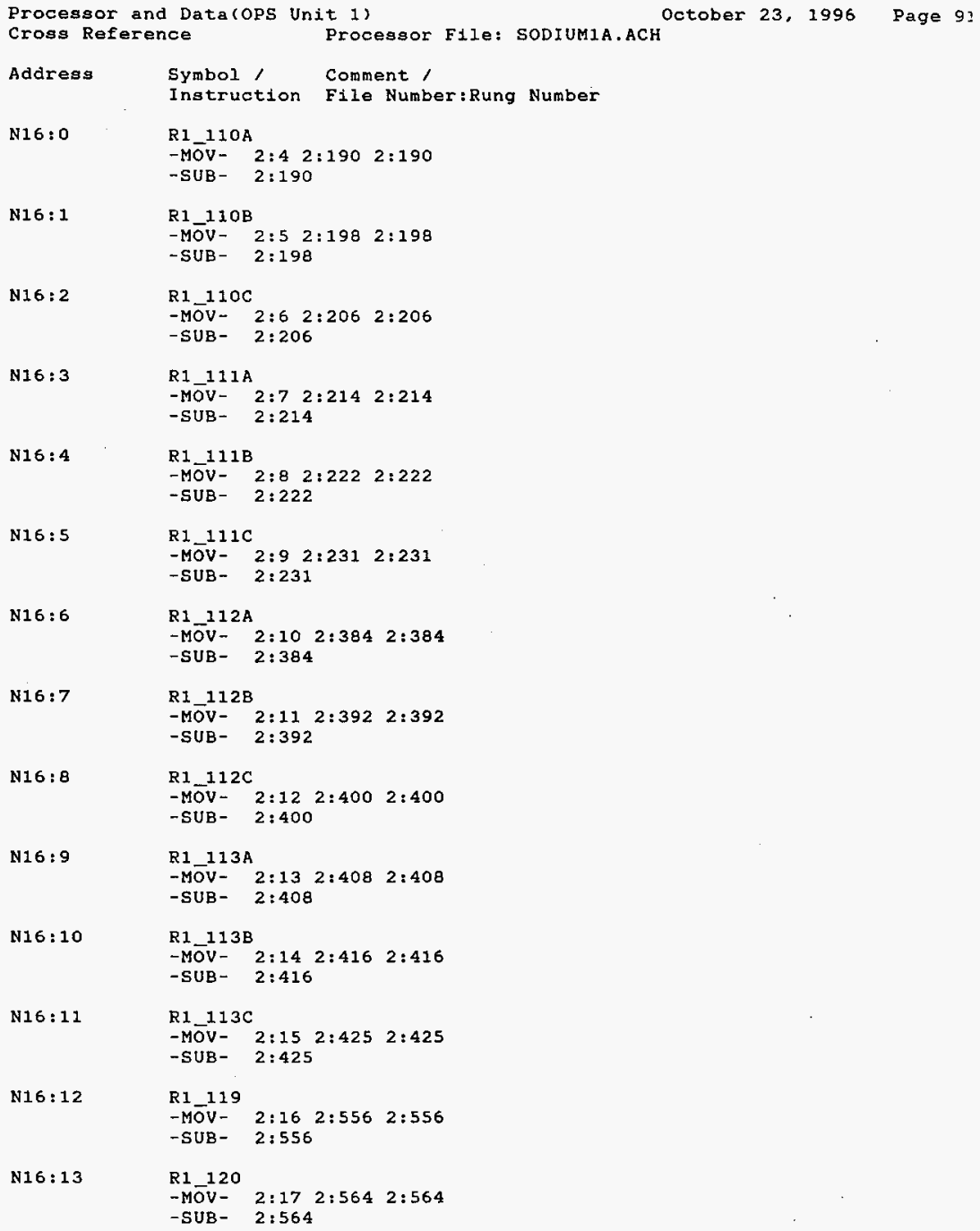

N16:12

R1 119

-MŌ- 2:16 2:556 2:556

-SUB- 2:556

N16:13

R1 120

-MOV- 2:17 2:564 2:564

SUB- 2:564

HNF-SD-FF-CSWD-60Rev. 0 


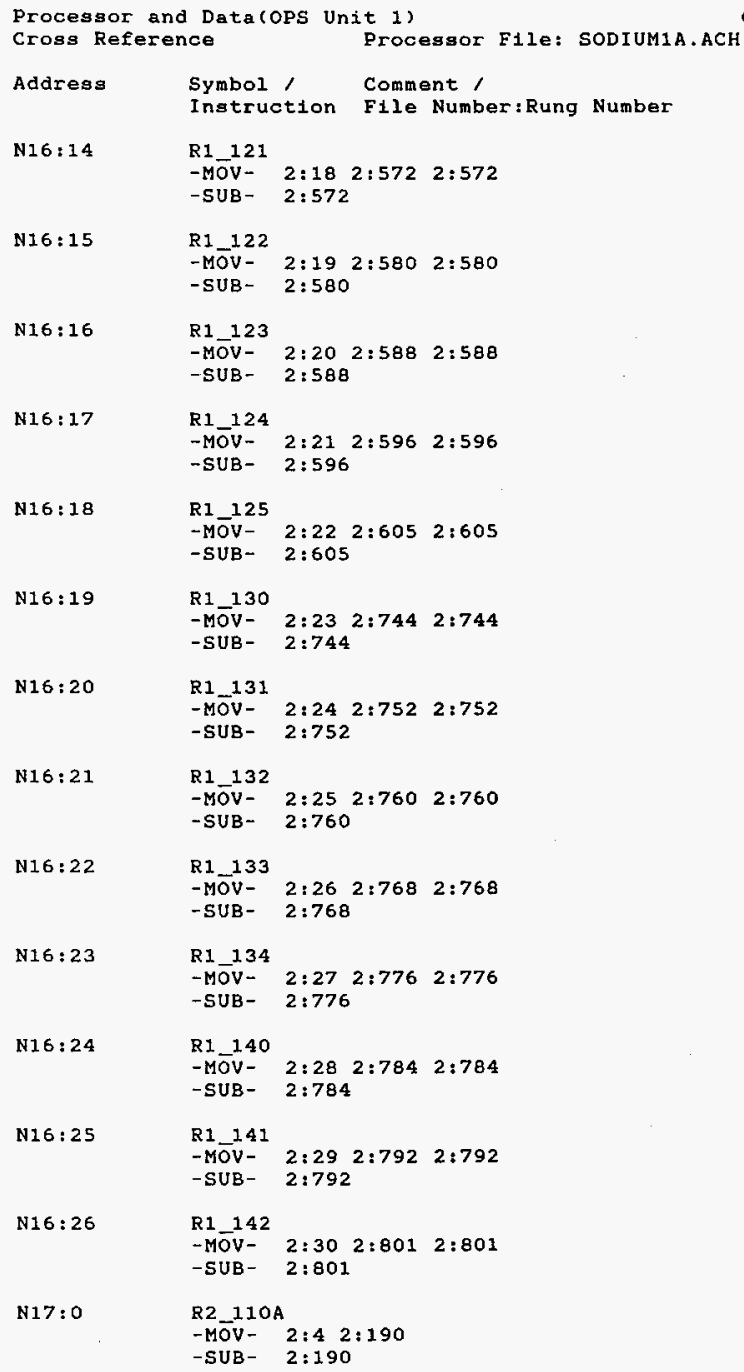

N1 $6: 23$

R1 134

-MOV- $2: 27 \quad 2: 776 \quad 2: 776$

-SUB- $2: 776$

N16: 24

R1 140

-MOV- 2:28 2:784 2:784

-SUB - $2: 784$

N16:25

R1_141

$\begin{array}{llll}\text { MOV- } & 2: 29 \quad 2: 792 \quad 2: 792\end{array}$

-SUB- $2: 792$

N16:26

R1 142

-MOV- $2: 30 \quad 2: 801 \quad 2: 801$

-SUB - 2:801.

N17:0

R2 110A

MOV- $2: 42: 190$

-SUB- 2:190

HNF-SD-FF-CSWD-60Rev. 0 


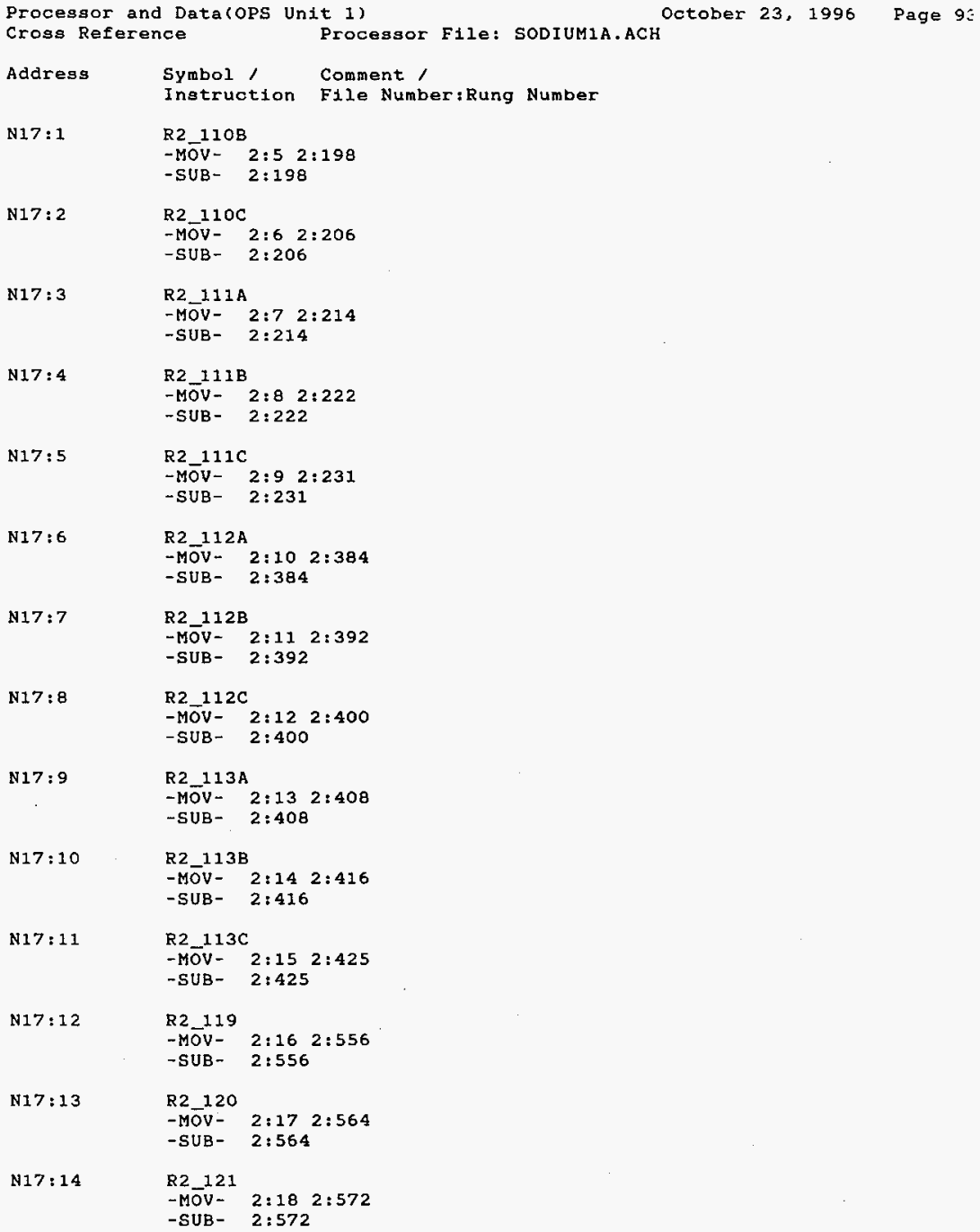

N17: 4

R2 111B

MOV- 2:8 2:222

-SUB - 2:222

N17:5

R2_111C

MOV- 2:9 2:231

-SUB- 2:231

N17:6

R2 112A

-MOV- 2:10 2:384

-SUB- $2: 384$

N17:7

R2 112B

-MOV- 2:11 2:392

-SUB- $2: 392$

N17:8

R2 $112 \mathrm{C}$

-MOV- 2:12 2:400

-SUB- 2:400

N17:9

R2 $113 \mathrm{~A}$

-MOV- 2:13 2:408

-SUB- 2:408

N17: 10

R2_113B

-MOV- 2:14 2:416

-SUB- $2: 416$

N17: 11

R2_113C

-MOV- 2:15 2:425

-SUB- 2:425

N17:12

R2 119

-MOV- 2:16 2:556

-SUB- 2:556

N17:13

R2 120

-MOV- 2:17 2:564

-SUB- 2:564

$\mathrm{N} 17: 14$

R2_121

- MOV- 2:18 2:572

-SUB- 2:572

HNF-SD-FF-CSWD-60 Rev. 0 


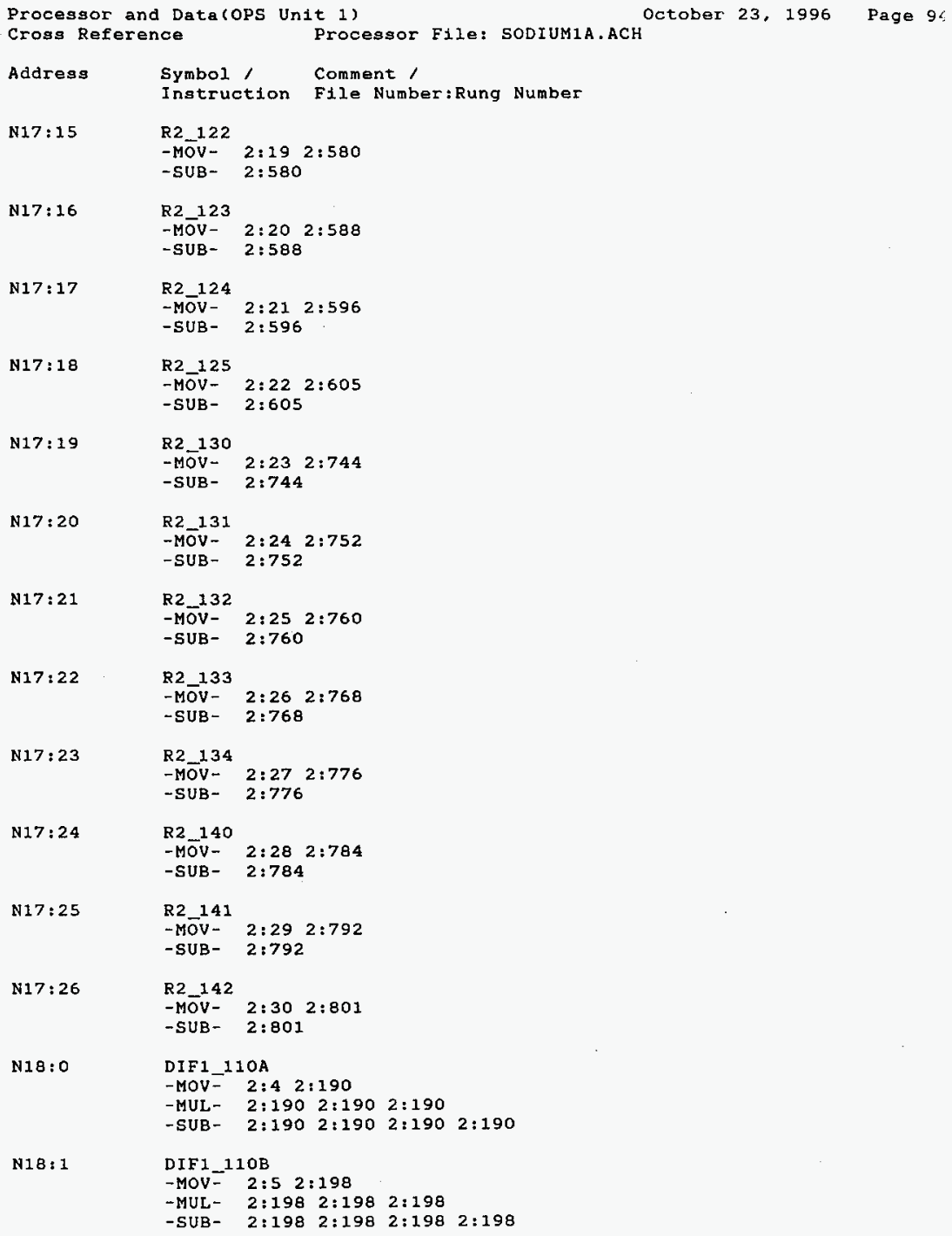

N18:0

DIF1 110A

-MOV- 2:4 2:190

-MUL- 2:190 2:190 2:190

-SUB- 2:190 2:190 2:190 2:190

N18: 1 
Processor and Data(OPS Unit 1 )

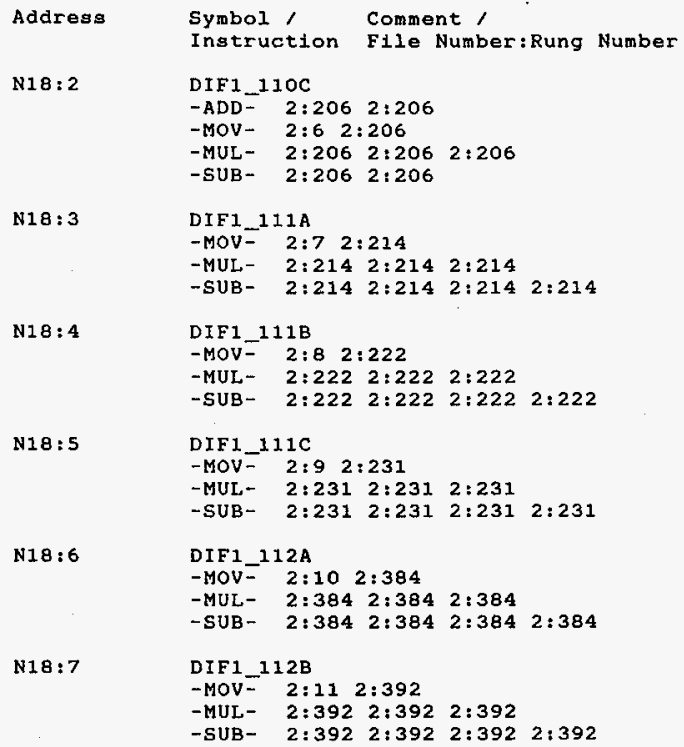

N18:3

DIF1_111A

-MOV= 2:7 2:214

-MUL- 2:214 2:214 2:214

-SUB- 2:214 2:214 2:214 2:214

N18:4

DIF1_111B

-MOV= 2:8 2:222

-MUL- $2: 222 \quad 2: 222 \quad 2: 222$

-SUB- 2:222 2:222 2:222 2:222

N18:5

DIF1_111C

-MOV- 2:9 2:231

-MUL_ $2: 231 \quad 2: 231 \quad 2: 231$

-SUB- 2:231 2:231 2:231 2:231

N18:6

DIF1_112A

-MOV= $2: 10 \quad 2: 384$

-MUL- 2:384 2:384 2:384

-SUB- $2: 384 \quad 2: 384 \quad 2: 384 \quad 2: 384$

$N 18: 7$

DIF1_112B

-MOV= 2:11 2:392

-MUL- $2: 392 \quad 2: 392 \quad 2: 392$

-SUB- 2:392 2:392 2:392 2:392

N18:8

DIF1_112C

-MOV= 2:12 2:400

-MUL- 2:400 2:400 2:400

-SUB- 2:400 2:400 2:400 2:400

N18: 9

DIF1_113A

-MOV- $2: 132: 408$

-MUL- 2:408 2:408 2:408

-sUB- 2:408 2:408 2:408 2:408

N18: 10

DIF1_113B

-MOV- 2:14 2:416

-MUL- 2:416 2:416 2:416

-SUB- 2:416 2:416 2:416 2:416

N18:11

DIF $1 \_113 \mathrm{C}$

MOV- 2:15 2:425

-MUL- 2:425 2:425 2:425

-SUB- 2:425 2:425 2:425 2:425

$\mathrm{N} 18: 12$

DIF1_119

-MOV= 2:16 2:556

-MUL- 2:556 2:556 2:556

-SUB- 2:556 2:556 2:556 2:556

HNF-SD-FF-CSWD-60 Rev. 0 


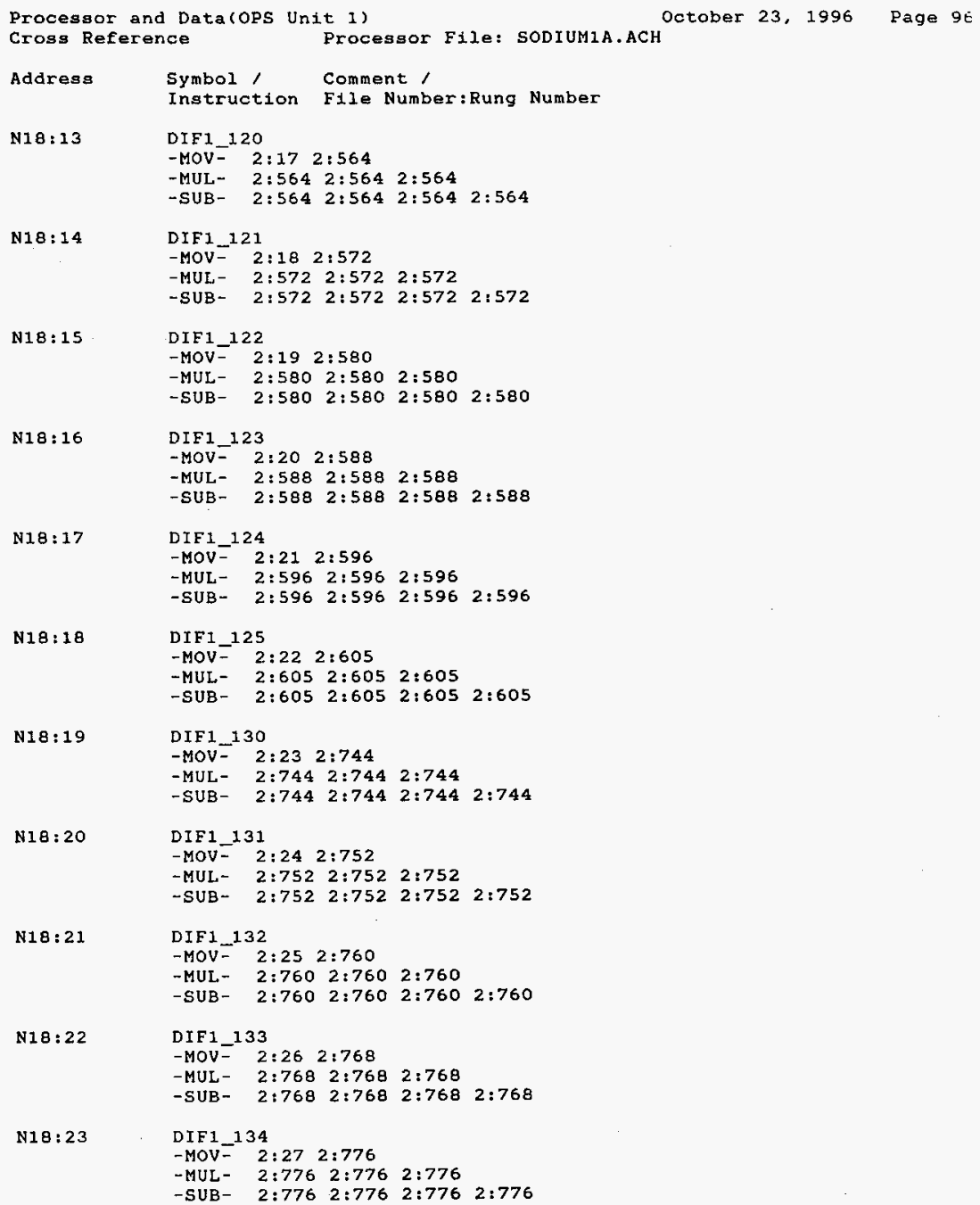

N18:23

DIF1_134

-MOV- $2: 27 \quad 2: 776$

-MUL- $2: 776 \quad 2: 776 \quad 2: 776$

-suB- 2:776 2:776 2:776 2:776

HNF-SD-FF-CSWD-60Rev. 0 


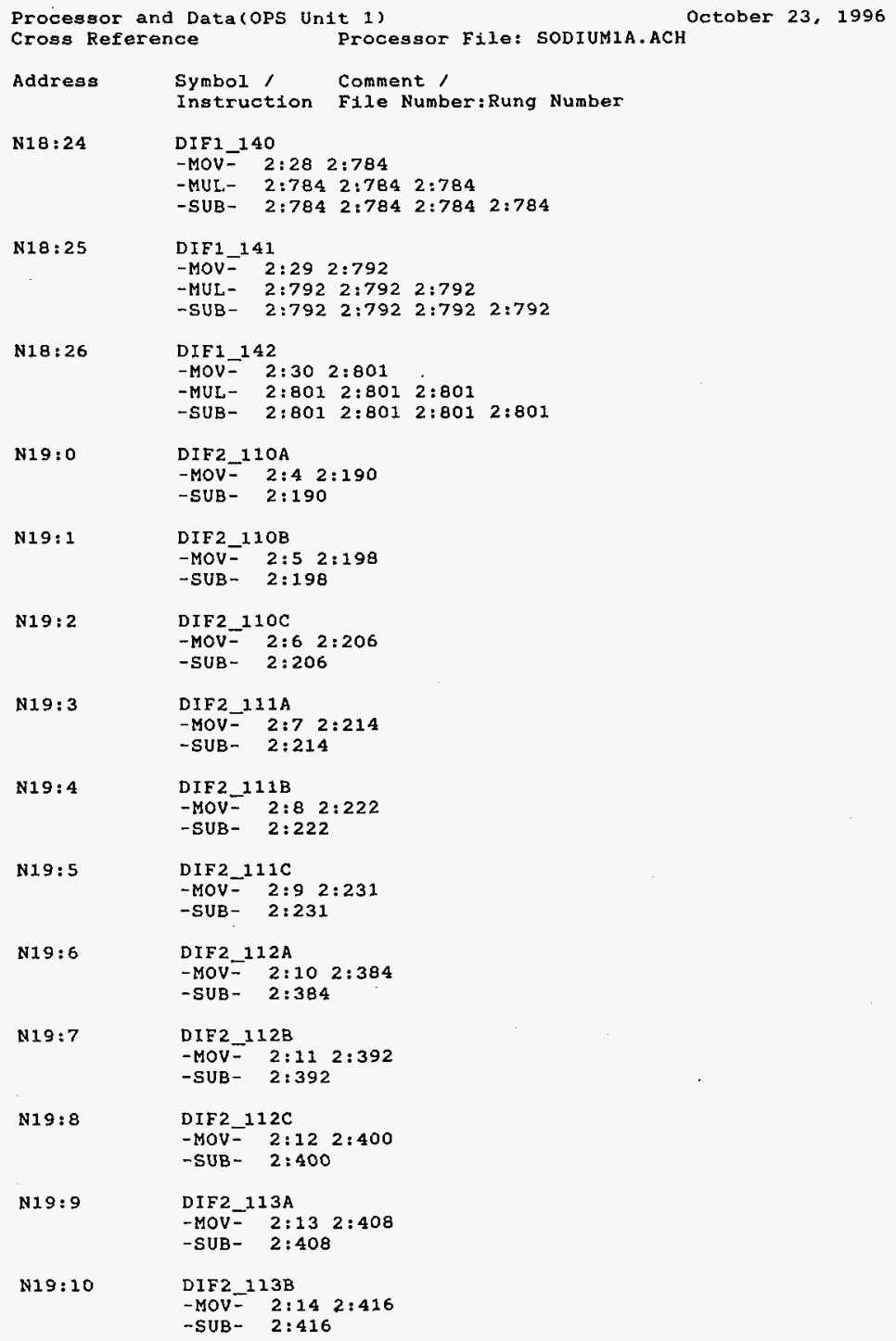

N19: 2

DIF 2_110C

-MOV= 2:6 2:206

-SU8- 2:206

N19:3

DIF $2111 \mathrm{~A}$

-MOV- $2: 7 \quad 2: 214$

-SUB- $2: 214$

N19: 4

DIF 2_111B

-MOV= 2:8 2:222

-SUB- $2: 222$

N19: 5

DIF2_111C

-MOV= 2:9 2:231

-SUB- 2:231

N19:6

DIF2 112A

-MOV- 2:10 2:384

-SUB- $2: 384$

N19:7

DIE2_112B

-MOV- 2:11 2:392

-SUB- 2:392

N19:8

DIF2_112C

-MOV- 2:12 2:400

-SUB- 2:400

N1 $9: 9$

DIF2 $113 \mathrm{~A}$

-MOV= 2:13 2:408

-SUB- 2:408

N19:10

$$
\begin{aligned}
& \text { DIF2 } 113 B \\
& \text {-MOV- } 2: 14 \quad 2: 416 \\
& \text {-SUB- } 2: 416
\end{aligned}
$$




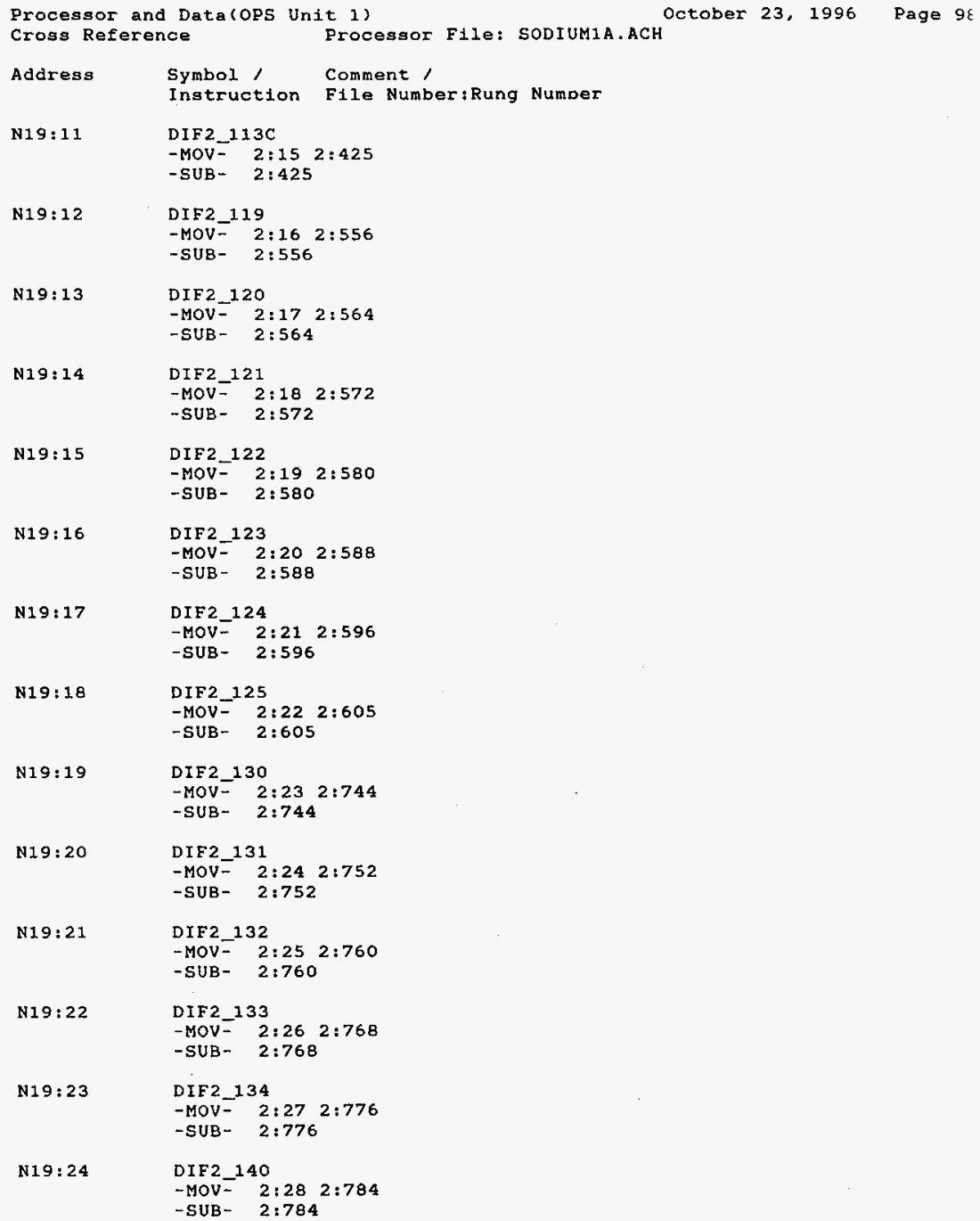

N19:22

DIF2_133

-MOV- 2:26 2:76B

-sUB- 2:768

N19:23

DIF 2,134

-MOV- 2:27 2:776

-SUB- 2:776

N19:24

DIF2 140

-MOV $=2: 28 \quad 2: 784$

-SUB- $2: 784$

HNF-SD-FF-CSWD-60 Rev. 0 
Processor and Data(OPs Unit 1 ) Cross Reference Processor File: SODIUM1A.ACH

Address

N19:25

N19:26

N20:0
Symbol, Comment ,

Instruction File Number:Rung Number

DIF2_141

- MOV= 2:29 2:792

-SUB- $2: 792$

DIF2_142

-MOV- 2:30 2:801

-SUB- 2:801

\section{ACC}

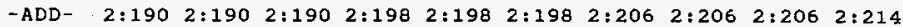

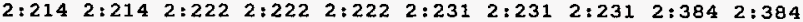

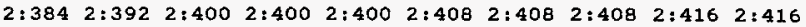
$\begin{array}{llllllllll}2: 416 & 2: 425 & 2: 425 & 2: 425 & 2: 556 & 2: 556 & 2: 556 & 2: 564 & 2: 564 & 2: 564\end{array}$

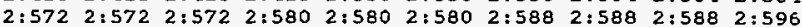
$\begin{array}{llllllllll}2: 596 & 2: 596 & 2: 605 & 2: 605 & 2: 605 & 2: 744 & 2: 744 & 2: 744 & 2: 752 & 2: 752\end{array}$ $\begin{array}{lllllllllll}2: 752 & 2: 760 & 2: 760 & 2: 760 & 2: 768 & 2: 768 & 2: 768 & 2: 776 & 2: 776 & 2: 776\end{array}$ $\begin{array}{lllllll}2: 784 & 2: 784 & 2: 784 & 2: 792 \quad 2: 792 \quad 2: 792 & 2: 801 & 2: 801 \quad 2: 801\end{array}$

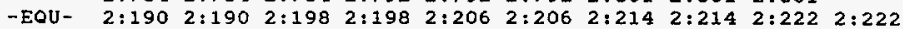

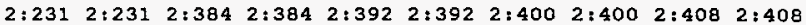

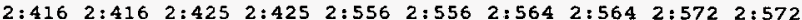

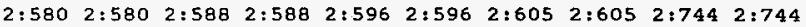
$\begin{array}{lllllllllll}2: 752 & 2: 752 & 2: 760 & 2: 760 & 2: 768 & 2: 768 & 2: 776 & 2: 776 & 2: 784 & 2: 784\end{array}$ $2: 792 \quad 2: 792 \quad 2: 801 \quad 2: 801$

$\begin{array}{lllllllllll}\text { GRT- } & 2: 190 & 2: 198 & 2: 206 & 2: 214 & 2: 222 & 2: 231 & 2: 384 & 2: 392 & 2: 400 & 2: 408\end{array}$

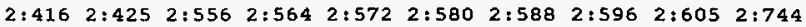
$2: 752 \quad 2: 760 \quad 2: 768 \quad 2: 776 \quad 2: 784 \quad 2: 792 \quad 2: 801$

-LES- $2: 190 \quad 2: 198 \quad 2: 206 \quad 2: 214 \quad 2: 222 \quad 2: 231 \quad 2: 384 \quad 2: 392 \quad 2: 400 \quad 2: 408$

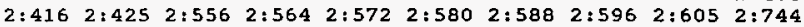
$\begin{array}{lllllll}2: 752 & 2: 760 & 2: 768 & 2: 776 & 2: 784 & 2: 792 & 2: 801\end{array}$

-LIM- $\quad 2: 190 \quad 2: 198 \quad 2: 206 \quad 2: 214 \quad 2: 222 \quad 2: 231 \quad 2: 384 \quad 2: 392 \quad 2: 400 \quad 2: 408$ $\begin{array}{llllllllll}2: 416 & 2: 425 & 2: 556 & 2: 564 & 2: 572 & 2: 580 & 2: 588 & 2: 596 & 2: 605 & 2: 744\end{array}$ $\begin{array}{llllll}2: 752 & 2: 760 & 2: 768 & 2: 776 & 2: 784 & 2: 792 \quad 2: 801\end{array}$

-MOV- $2: 190 \quad 2: 198 \quad 2: 206 \quad 2: 214 \quad 2: 222 \quad 2: 231 \quad 2: 384 \quad 2: 392 \quad 2: 400 \quad 2: 408$

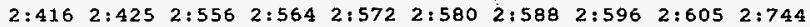
$\begin{array}{lllll}2: 752 & 2: 760 & 2: 768 & 2: 776 \quad 2: 784 & 2: 792 \quad 2: 801\end{array}$

-MUL- $\quad 2: 190 \quad 2: 190 \quad 2: 190 \quad 2: 198 \quad 2: 198 \quad 2: 198 \quad 2: 206 \quad 2: 206 \quad 2: 206 \quad 2: 214$ $\begin{array}{llllllllll}2: 214 & 2: 214 & 2: 222 & 2: 222 & 2: 222 & 2: 231 & 2: 231 & 2: 231 & 2: 384 & 2: 384\end{array}$

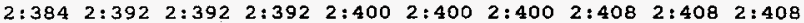

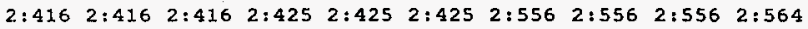

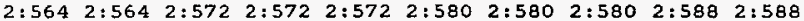
$\begin{array}{llllllllll}2: 588 & 2: 596 & 2: 596 & 2: 596 & 2: 605 & 2: 605 & 2: 605 & 2: 744 & 2: 744 & 2: 744\end{array}$ $\begin{array}{lllllllllll}2: 752 & 2: 752 & 2: 752 & 2: 760 & 2: 760 & 2: 760 & 2: 768 & 2: 768 & 2: 768 & 2: 776\end{array}$ $\begin{array}{lllllllllll}2: 776 & 2: 776 & 2: 784 & 2: 784 & 2: 784 & 2: 792 & 2: 792 & 2: 792 & 2: 801 & 2: 801\end{array}$ $2: 801$

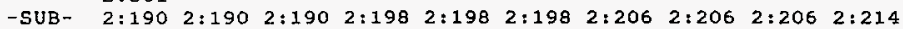

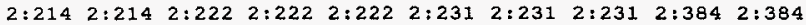

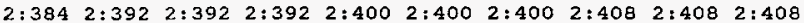

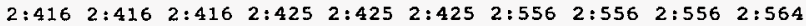

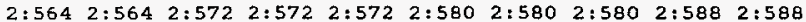
$\begin{array}{llllllllll}2: 588 & 2: 596 & 2: 596 & 2: 596 & 2: 605 & 2: 605 & 2: 605 & 2: 744 & 2: 744 & 2: 744\end{array}$ $\begin{array}{llllllllllll}2: 752 & 2: 752 & 2: 752 & 2: 760 & 2: 760 & 2: 760 & 2: 768 & 2: 768 & 2: 768 & 2: 776\end{array}$ $\begin{array}{llllllllll}2: 776 & 2: 776 & 2: 784 & 2: 784 & 2: 784 & 2: 792 & 2: 792 & 2: 792 & 2: 801 & 2: 801\end{array}$ $2: 801$

HNF-SD-FF-CSWD- 60 Rev. 0 
Processor and Data(OPS Unit 1 ) Cross Reference

Processor File: SODIUMIA.ACH

October 23, 1996 Page 10

\begin{tabular}{|c|c|c|c|c|c|c|c|c|c|c|c|}
\hline Address & $\begin{array}{l}\text { Symbo } \\
\text { Instr }\end{array}$ & tion & $\begin{array}{l}\text { Commer } \\
\text { File }\end{array}$ & $\begin{array}{l}t / \\
\text { umber }\end{array}$ & Rung 1 & Number & & & & & \\
\hline N20: 1 & $\mathrm{ACC} 1$ & & & & & & & & & & \\
\hline & -ADD- & $2: 190$ & $2: 190$ & $2: 198$ & $2: 198$ & $2: 206$ & $2: 206$ & $2: 214$ & $2: 214$ & $2: 222$ & $2: 222$ \\
\hline & & $2: 231$ & $2: 231$ & $2: 384$ & $2: 384$ & $2: 392$ & $2: 392$ & $2: 400$ & $2: 400$ & $2: 408$ & $2: 408$ \\
\hline & & $2: 416$ & $2: 416$ & $2: 425$ & $2: 425$ & $2: 556$ & $2: 556$ & $2: 564$ & $2: 564$ & $2: 572$ & $2: 572$ \\
\hline & & $2: 580$ & $2: 580$ & $2: 588$ & $2: 588$ & $2: 596$ & $2: 596$ & $2: 605$ & $2: 605$ & $2: 744$ & $2: 744$ \\
\hline & & $\begin{array}{l}2: 752 \\
2: 792\end{array}$ & $\begin{array}{l}2: 752 \\
2: 792\end{array}$ & $\begin{array}{l}2: 760 \\
2: 801\end{array}$ & $\begin{array}{l}2: 760 \\
2: 801\end{array}$ & $2: 768$ & $2: 768$ & $2: 776$ & $2: 776$ & $2: 784$ & $2: 784$ \\
\hline & -MUL- & $2: 190$ & $2: 190$ & $2: 190$ & $2: 198$ & $2: 198$ & $2: 198$ & $2: 206$ & $2: 206$ & $2: 206$ & $2: 214$ \\
\hline & & $2: 214$ & $2: 214$ & $2: 222$ & $2: 222$ & $2: 222$ & $2: 231$ & $2: 231$ & $2: 231$ & $2: 384$ & $2: 384$ \\
\hline & & $2: 384$ & $2: 392$ & $2: 392$ & $2: 392$ & $2: 400$ & $2: 400$ & $2: 400$ & $2: 408$ & $2: 408$ & $2: 408$ \\
\hline & & $\begin{array}{l}2: 416 \\
2: 564\end{array}$ & $\begin{array}{l}2: 416 \\
2: 564\end{array}$ & $\begin{array}{l}2: 416 \\
2: 572\end{array}$ & $\begin{array}{l}2: 425 \\
2: 572\end{array}$ & $\begin{array}{l}2: 425 \\
2: 572\end{array}$ & $\begin{array}{l}2: 425 \\
2: 580\end{array}$ & $\begin{array}{l}2: 556 \\
2: 580\end{array}$ & $\begin{array}{l}2: 556 \\
2: 580\end{array}$ & $\begin{array}{l}2: 556 \\
2: 588\end{array}$ & $\begin{array}{l}2: 564 \\
2: 588\end{array}$ \\
\hline & & $\begin{array}{l}2: 588 \\
2: 752\end{array}$ & $\begin{array}{l}2: 596 \\
2: 752\end{array}$ & $\begin{array}{l}2: 596 \\
2: 752\end{array}$ & $\begin{array}{l}2: 596 \\
2: 760\end{array}$ & $\begin{array}{l}2: 605 \\
2: 760\end{array}$ & $\begin{array}{l}2: 605 \\
2: 760\end{array}$ & $\begin{array}{l}2: 605 \\
2: 768\end{array}$ & $\begin{array}{l}2: 744 \\
2: 768\end{array}$ & $\begin{array}{l}2: 744 \\
2: 768\end{array}$ & $\begin{array}{l}2: 744 \\
2: 776\end{array}$ \\
\hline & & $\begin{array}{l}2: 776 \\
2: 801\end{array}$ & $2: 776$ & $2: 784$ & $2: 784$ & $2: 784$ & $2: 792$ & $2: 792$ & $2: 792$ & $2: 801$ & $2: 801$ \\
\hline & -SUB- & $\begin{array}{l}2: 190 \\
2: 416 \\
2: 752\end{array}$ & $\begin{array}{l}2: 198 \\
2: 425 \\
2: 760\end{array}$ & $\begin{array}{l}2: 206 \\
2: 556 \\
2: 768\end{array}$ & $\begin{array}{l}2: 214 \\
2: 564 \\
2: 776\end{array}$ & $\begin{array}{l}2: 222 \\
2: 572 \\
2: 784\end{array}$ & $\begin{array}{l}2: 231 \\
2: 580 \\
2: 792\end{array}$ & $\begin{array}{l}2: 384 \\
2: 588 \\
2: 801\end{array}$ & $\begin{array}{l}2: 392 \\
2: 596\end{array}$ & $\begin{array}{l}2: 400 \\
2: 605\end{array}$ & $\begin{array}{l}2: 408 \\
2: 744\end{array}$ \\
\hline
\end{tabular}

N20:2 DDIF

-MUL- $2: 190 \quad 2: 190 \quad 2: 190 \quad 2: 198 \quad 2: 198 \quad 2: 198 \quad 2: 206 \quad 2: 206 \quad 2: 206 \quad 2: 214$

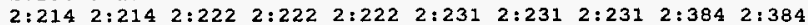

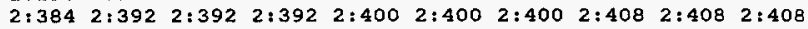

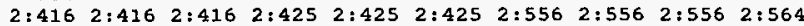

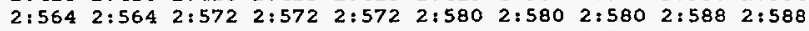
$\begin{array}{llllllllll}2: 588 & 2: 596 & 2: 596 & 2: 596 & 2: 605 & 2: 605 & 2: 605 & 2: 744 & 2: 744 & 2: 744\end{array}$ $\begin{array}{lllllllllll}2: 752 & 2: 752 & 2: 752 & 2: 760 & 2: 760 & 2: 760 & 2: 768 & 2: 768 & 2: 768 & 2: 776\end{array}$ $\begin{array}{llllllllll}2: 776 & 2: 776 & 2: 784 & 2: 784 & 2: 784 & 2: 792 & 2: 792 & 2: 792 & 2: 801 & 2: 801\end{array}$ 2:801

-suB- $2: 190 \quad 2: 198 \quad 2: 206 \quad 2: 214 \quad 2: 222 \quad 2: 231 \quad 2: 384 \quad 2: 392 \quad 2: 400 \quad 2: 408$

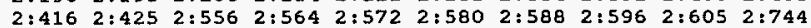
2:752 2:760 2:768 2:776 2:784 2:792 2:801

$\mathrm{N} 20: 3$

DDIF 1

-CLR -

$\begin{array}{lllllllllllll}2: 4 & 2: 5 & 2: 6 & 2: 7 & 2: 8 & 2: 9 & 2: 10 & 2: 11 & 2: 12 & 2: 13 & 2: 14 & 2: 15 & 2: 16\end{array}$

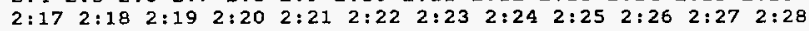
$2: 292: 30$

$\mathrm{N2O}: 4 \quad$ DDIF2

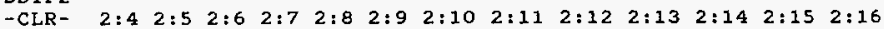

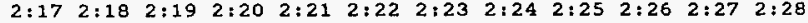
$2: 292: 30$

$\begin{array}{ll}N 20: 5 / 1 & D N-1 \\ & -][-2: 34 \\ & -] /[-2: 35 \\ & -(L)-2: 41 \\ & -(U)-2: 31 \quad 2: 34\end{array}$

N20:5/2

$$
\begin{array}{ll}
\text { DN_2 } & \\
-][- & 2: 34 \\
-] /[- & 2: 36 \\
-(L)- & 2: 239 \\
-(U)- & 2: 31 \quad 2: 34
\end{array}
$$




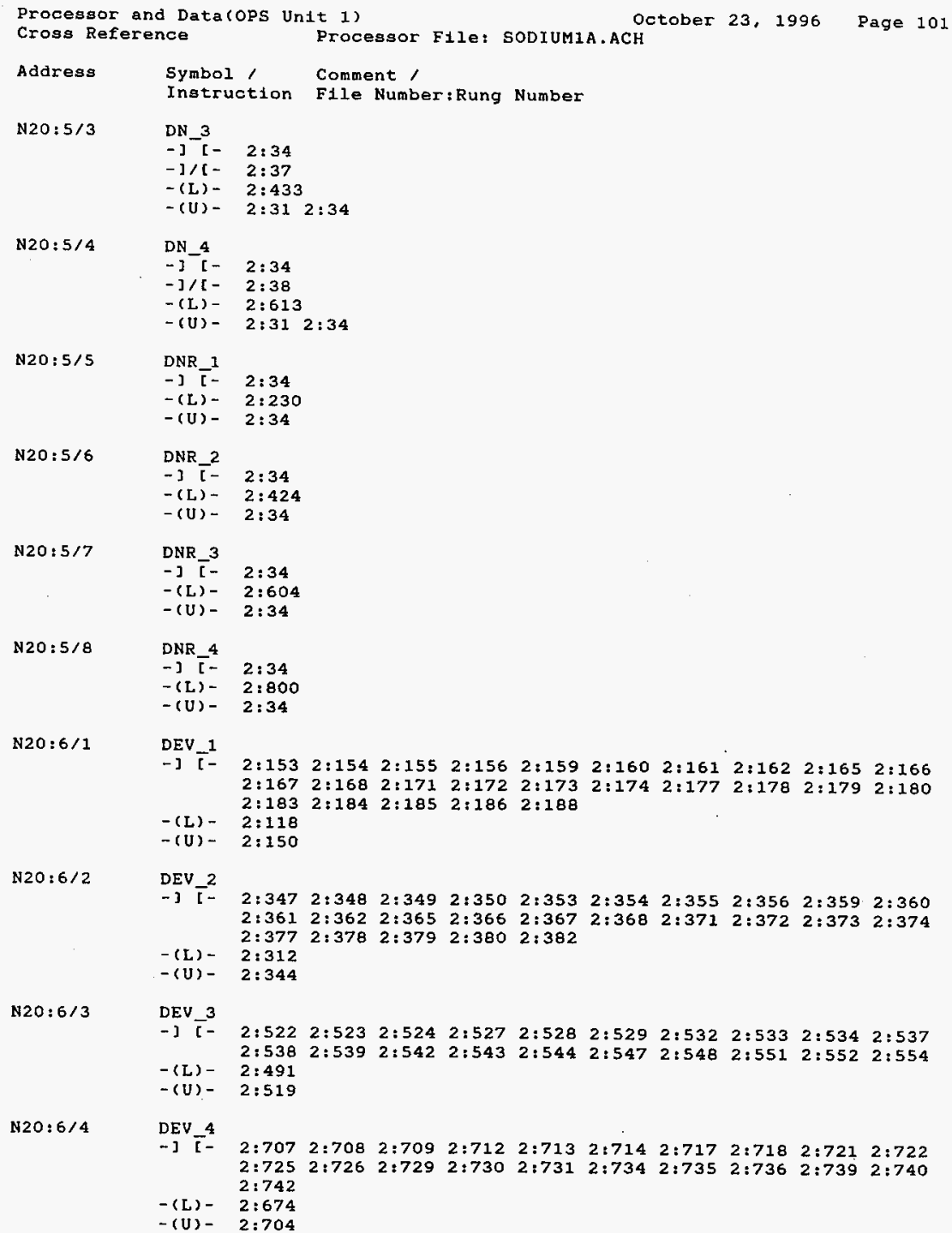

DNR_3

- ] $[-2: 34$

-(L.) - $2: 604$

$-(U)-2: 34$

N20:5/8

DNR_ 4

$-] \overline{[}-2: 34$

$-(\mathrm{L})-2: 800$

$-(U)-2: 34$

N20:6/1

DEV 1

N20:6/2

N20:6/3

HNF-SD-FF-CSWD-60 Rev. 0 


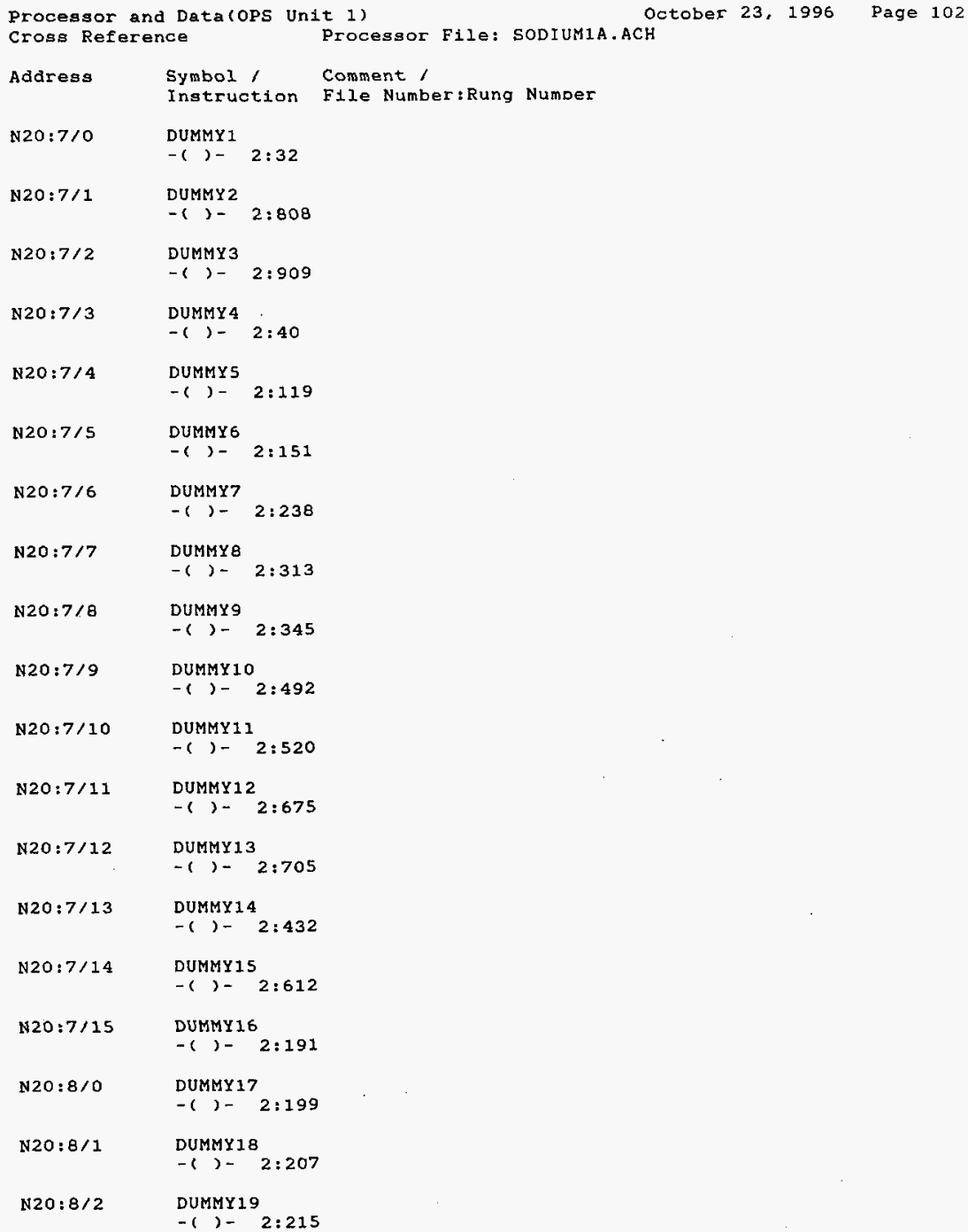




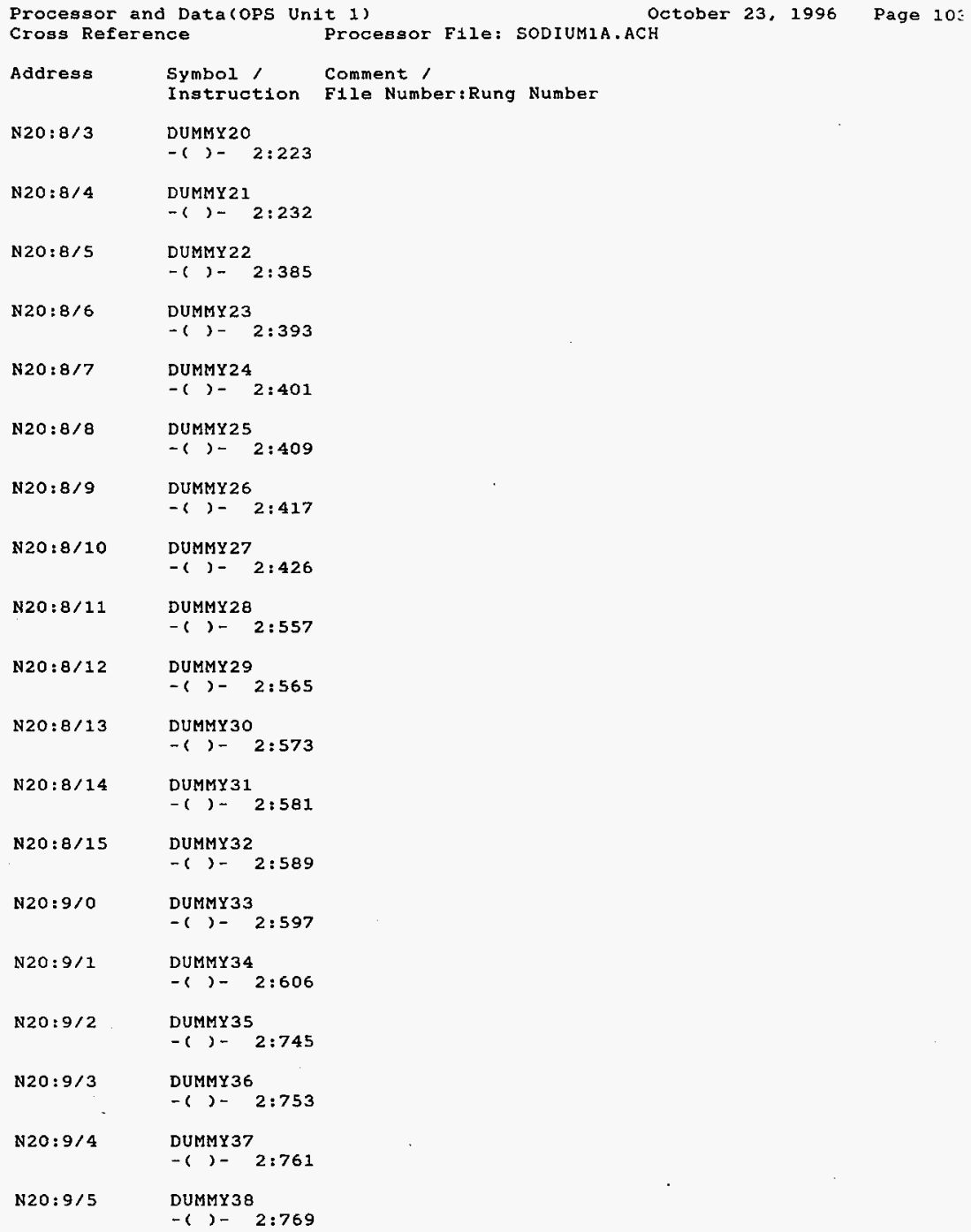




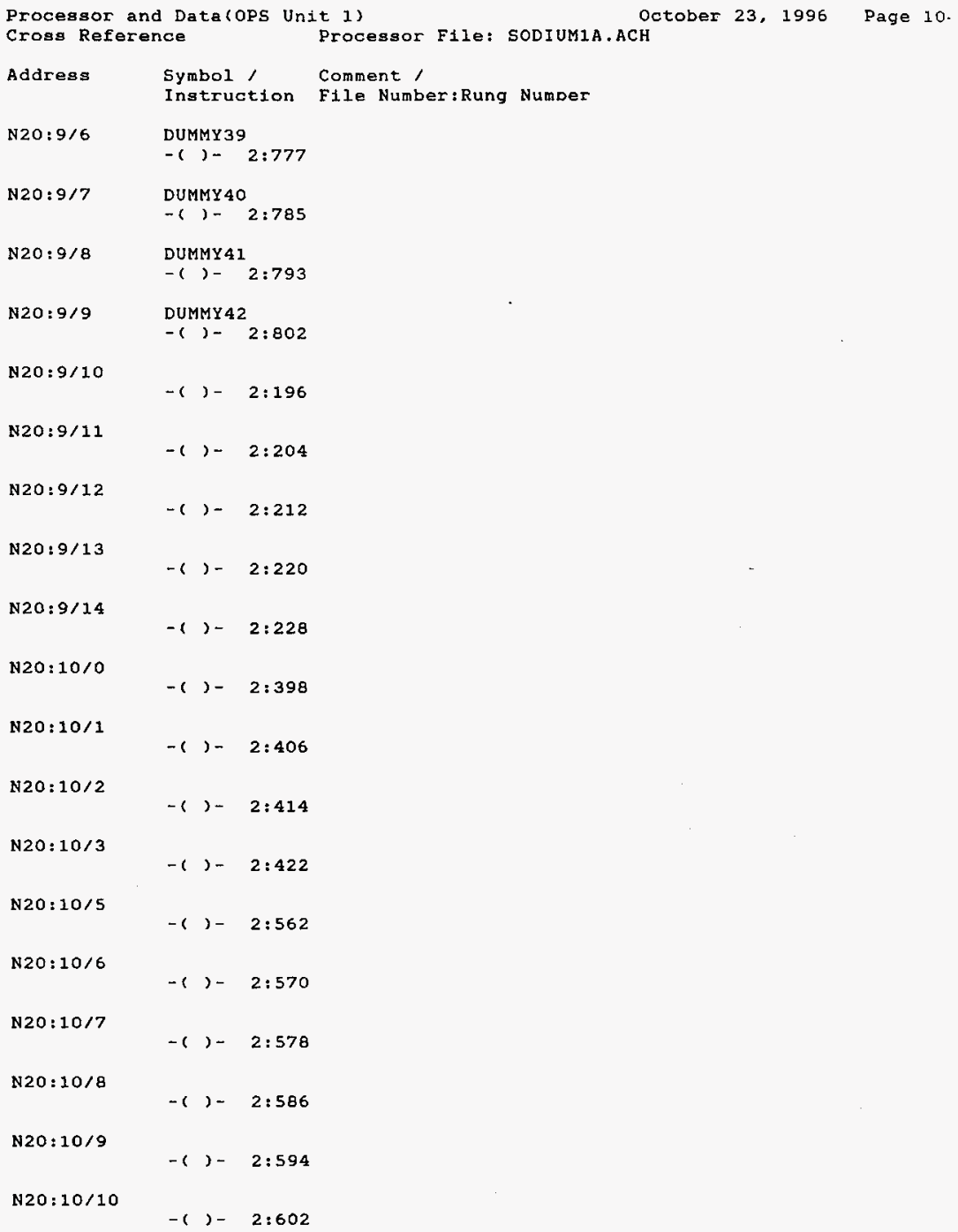

HNF-SD-FF-CSWD-60 Rev. 0 


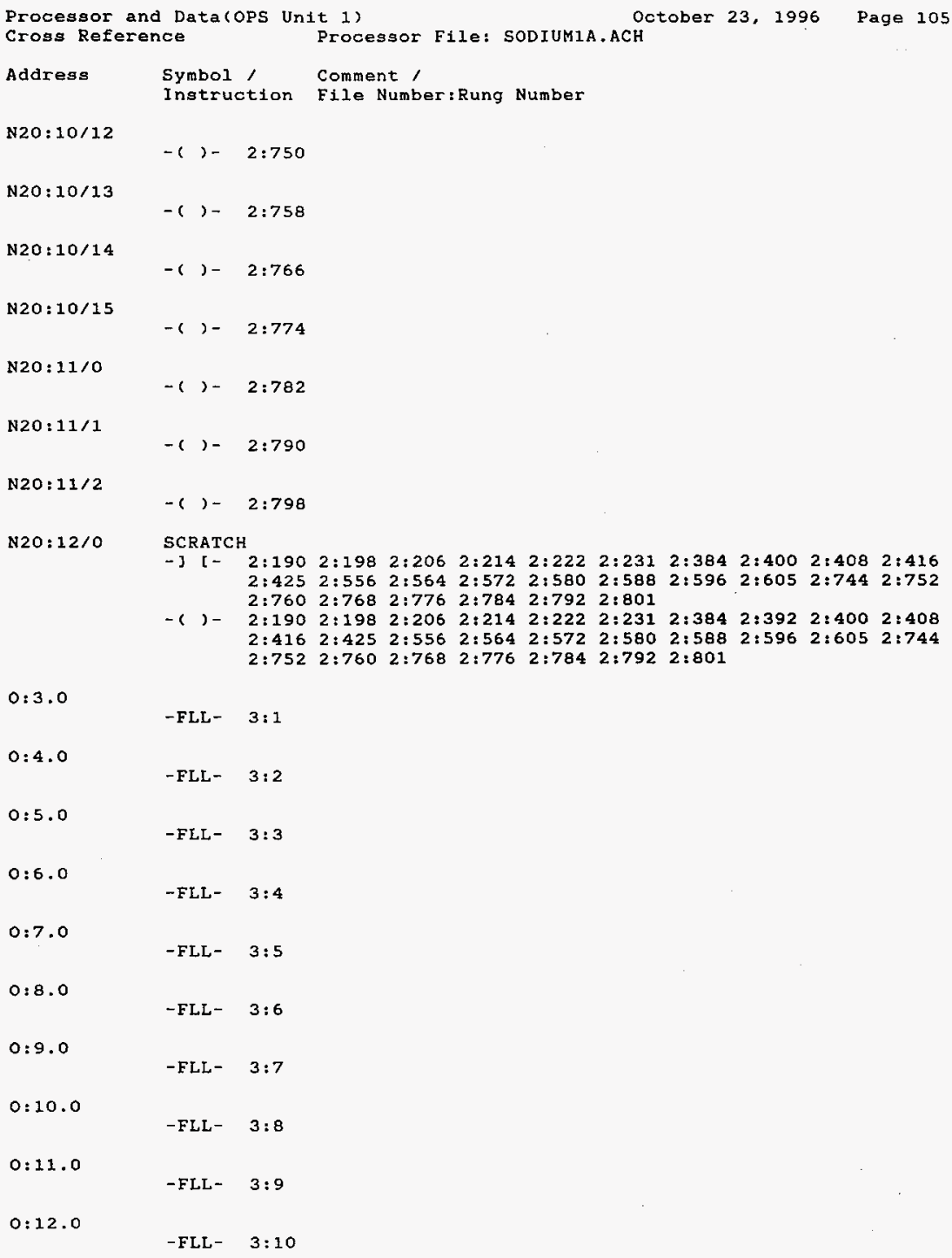




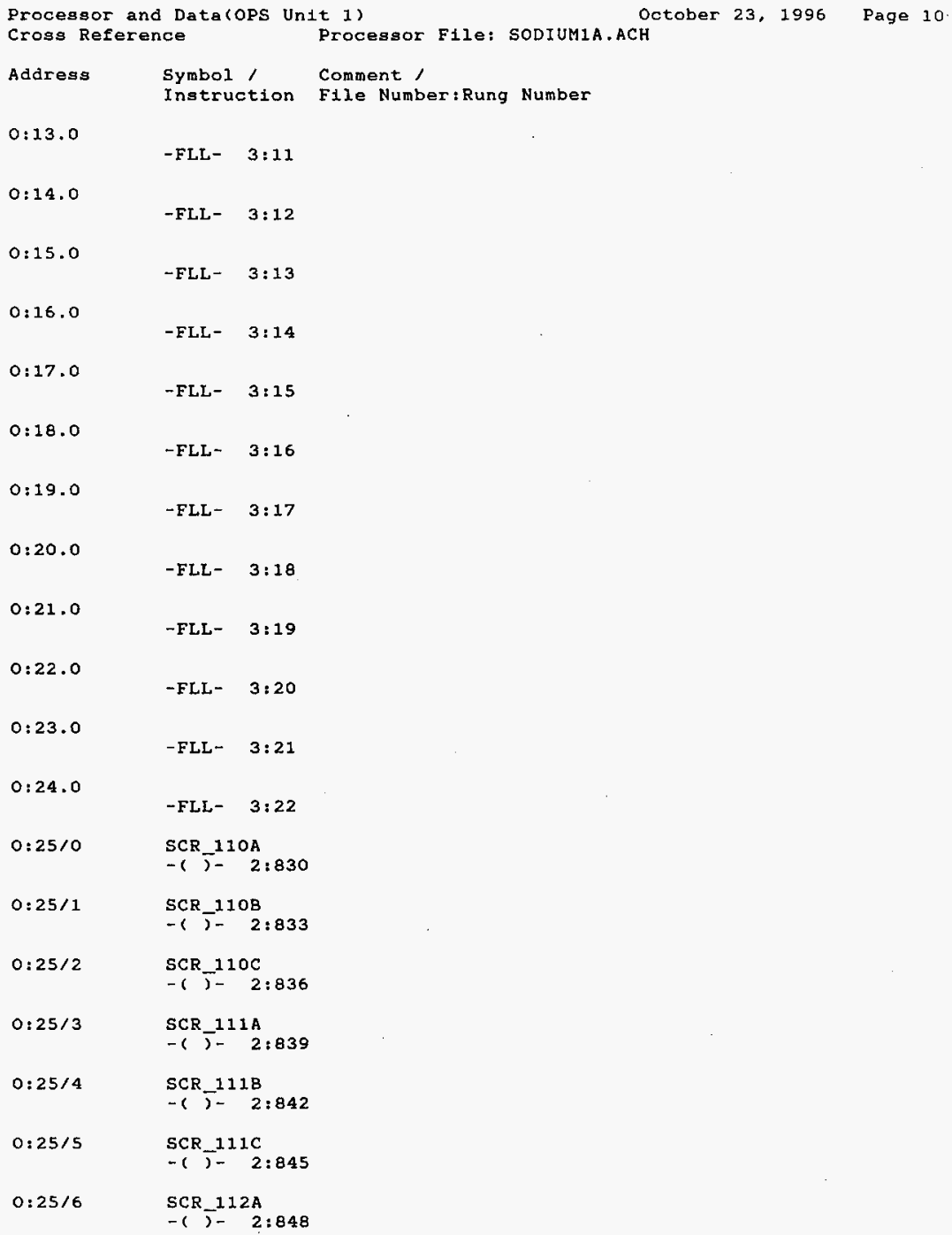

HNF-SD-FF-CSWD-60Rev. 0 


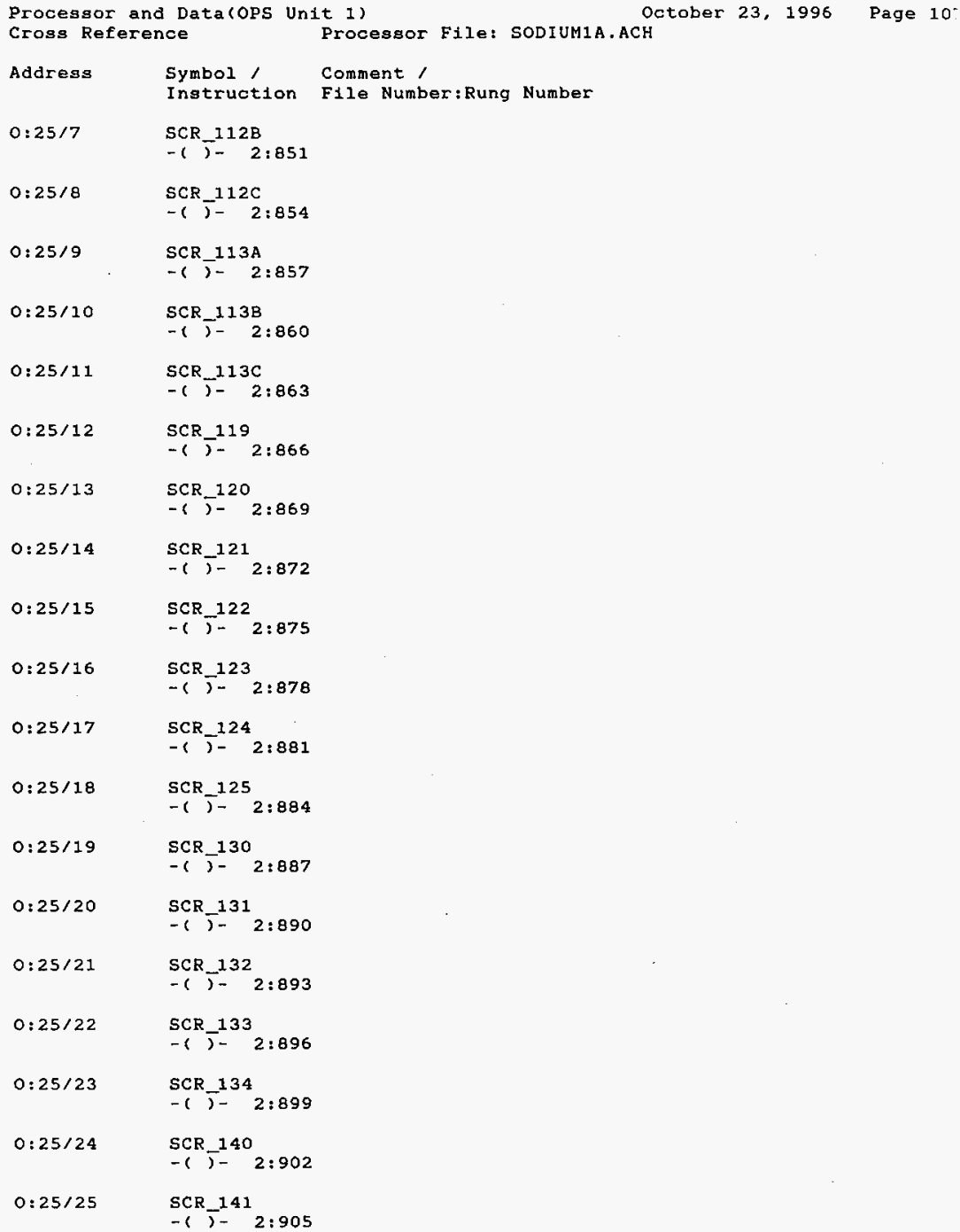

HNF-SD-FF-CSWD-60Rev. 0 


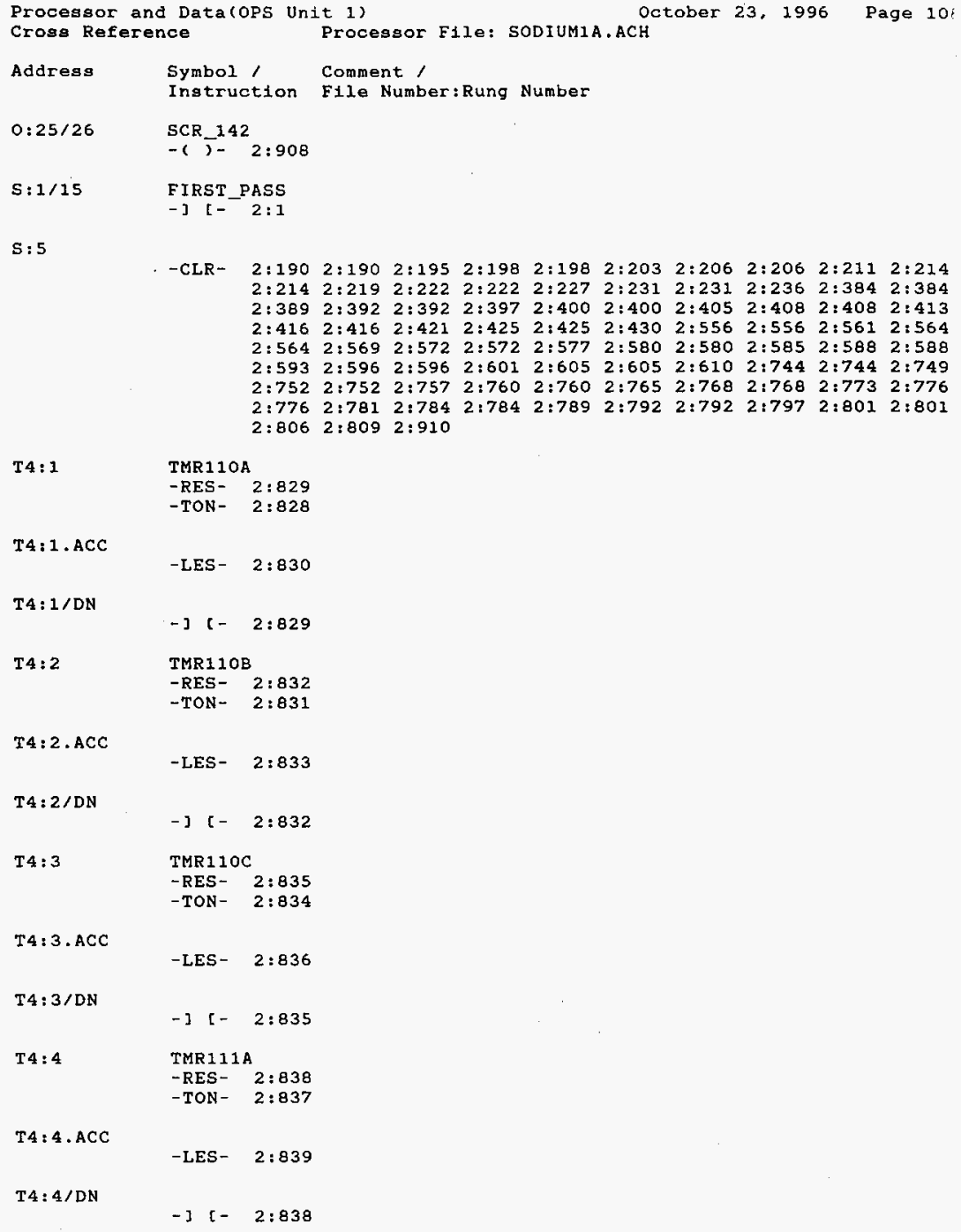

$T 4: 3 . A C C$

-LES- $2: 836$

T4:3/DN

$-][-2: 835$

T $4: 4$

TMR IIIA

-RES- $2: 838$

-TON- 2:837

$T 4: 4 . A C C$

-LES- $\quad 2: 839$

T4 : 4/DN

$$
\text { - ] }[-2: 838
$$




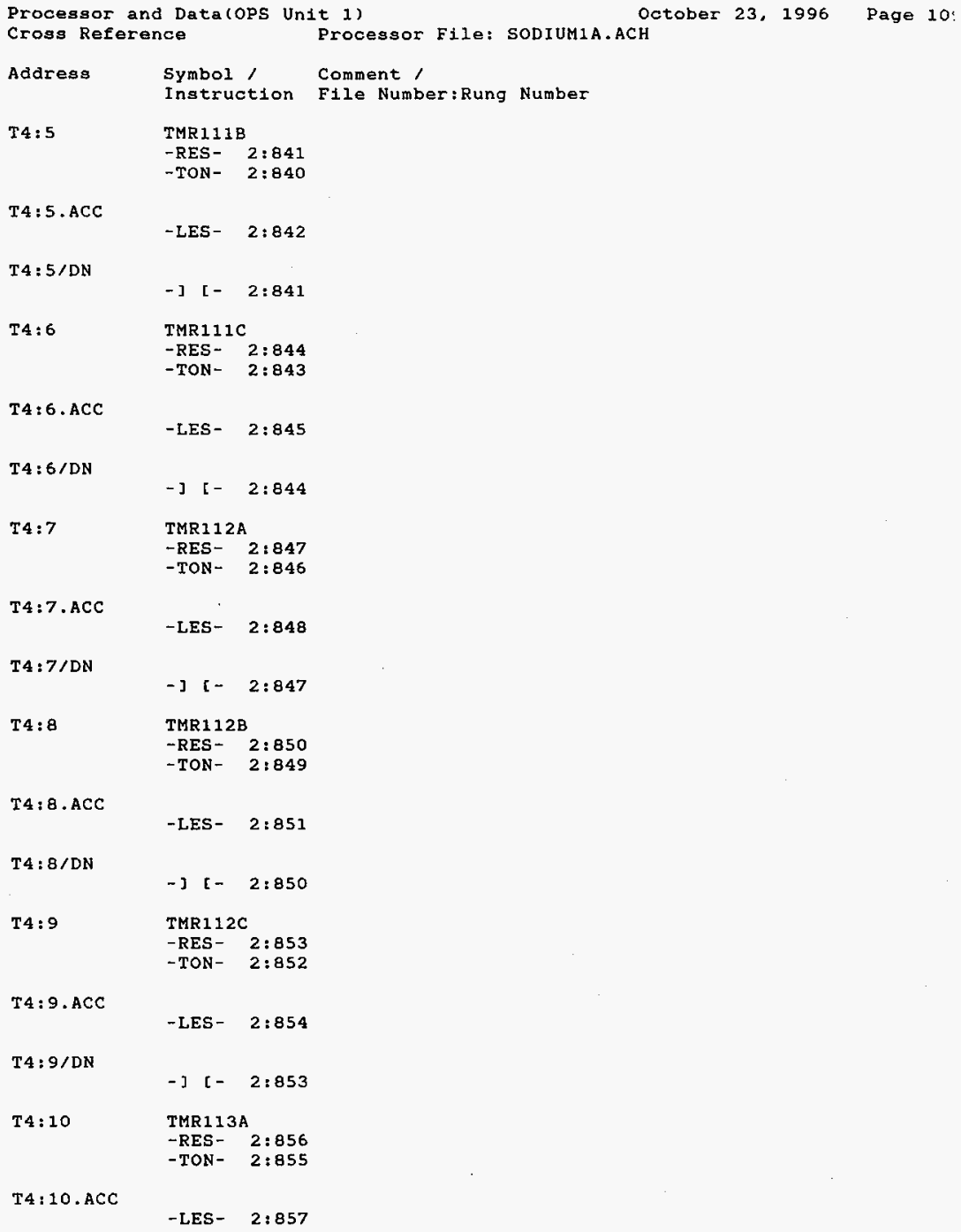

HNF-SD-FF-CSWD-60Rev. 0 


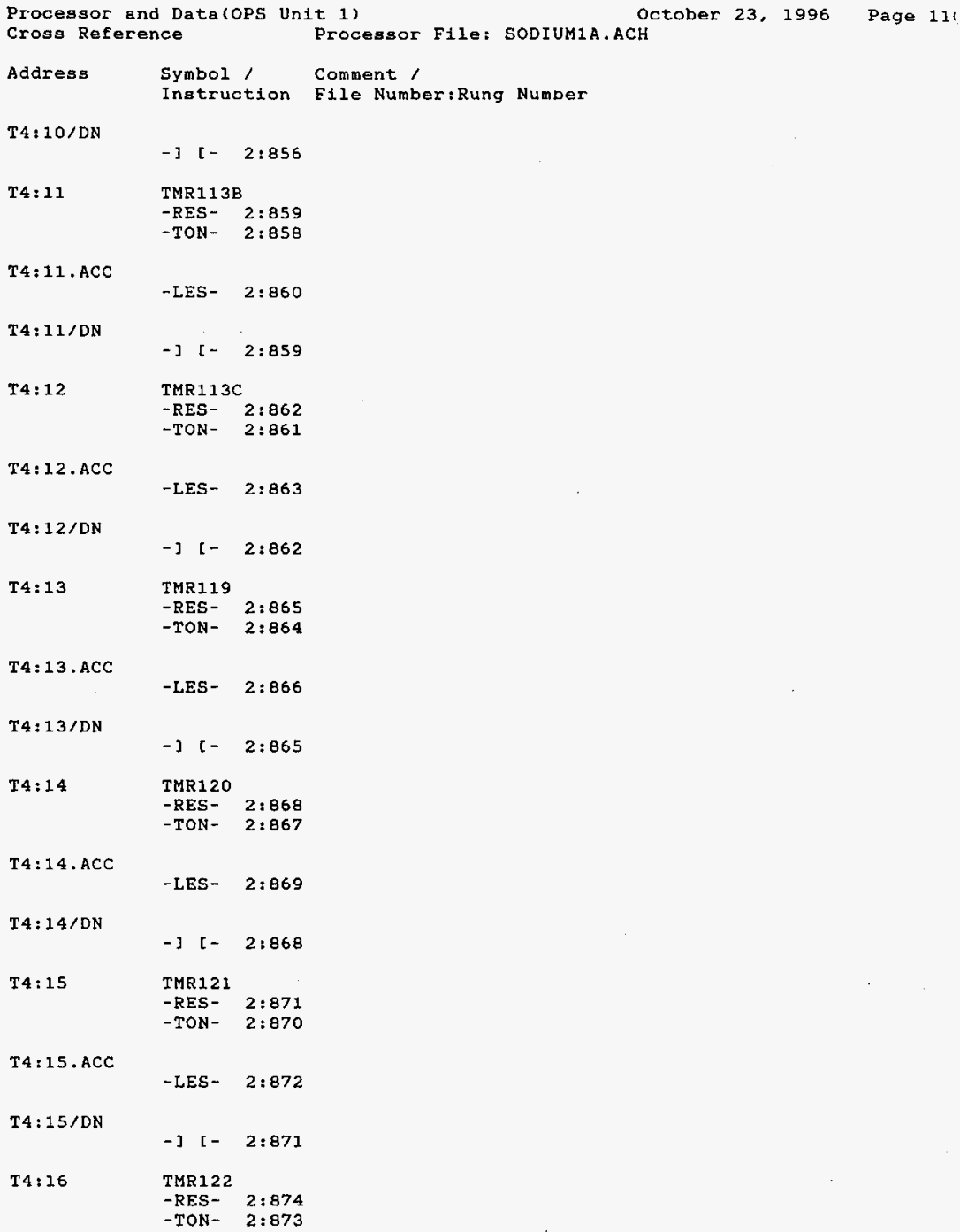

HNF-SD-FF-CSWD-60Rev. 0 


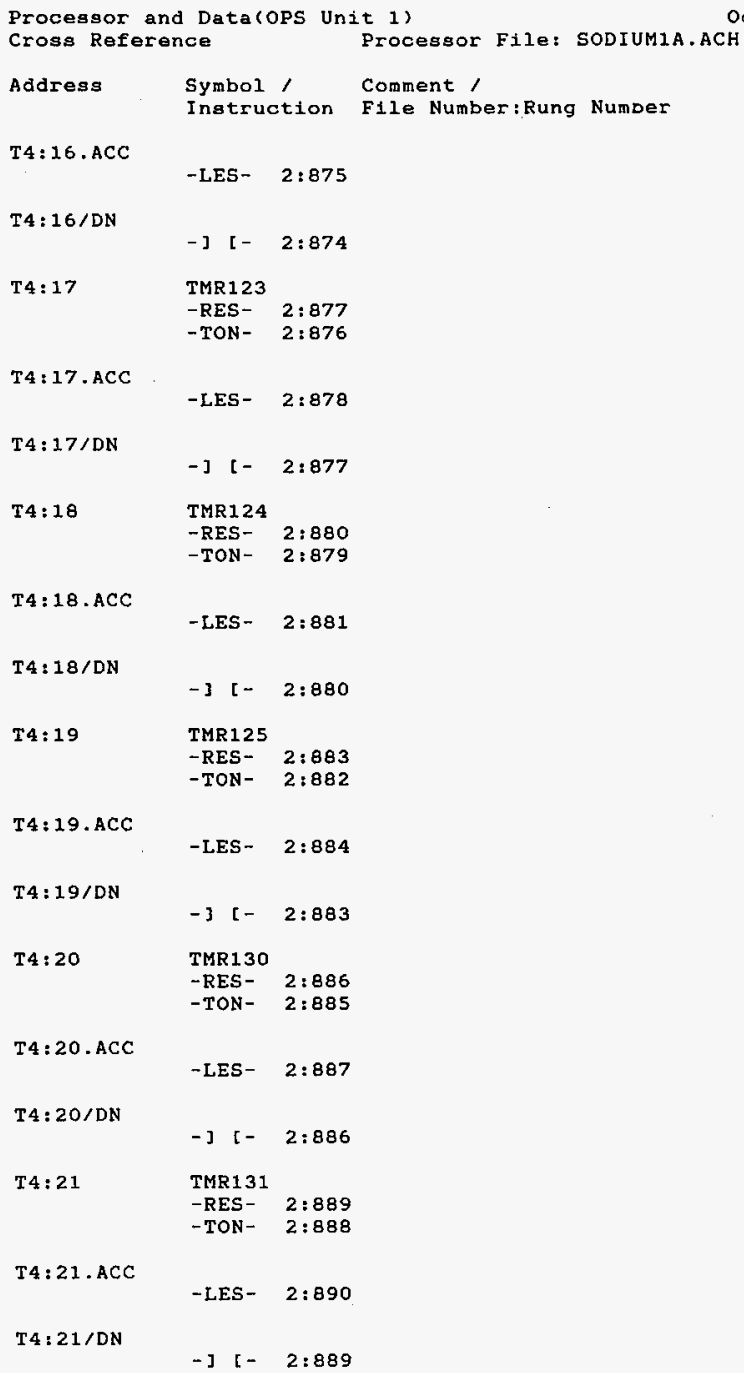




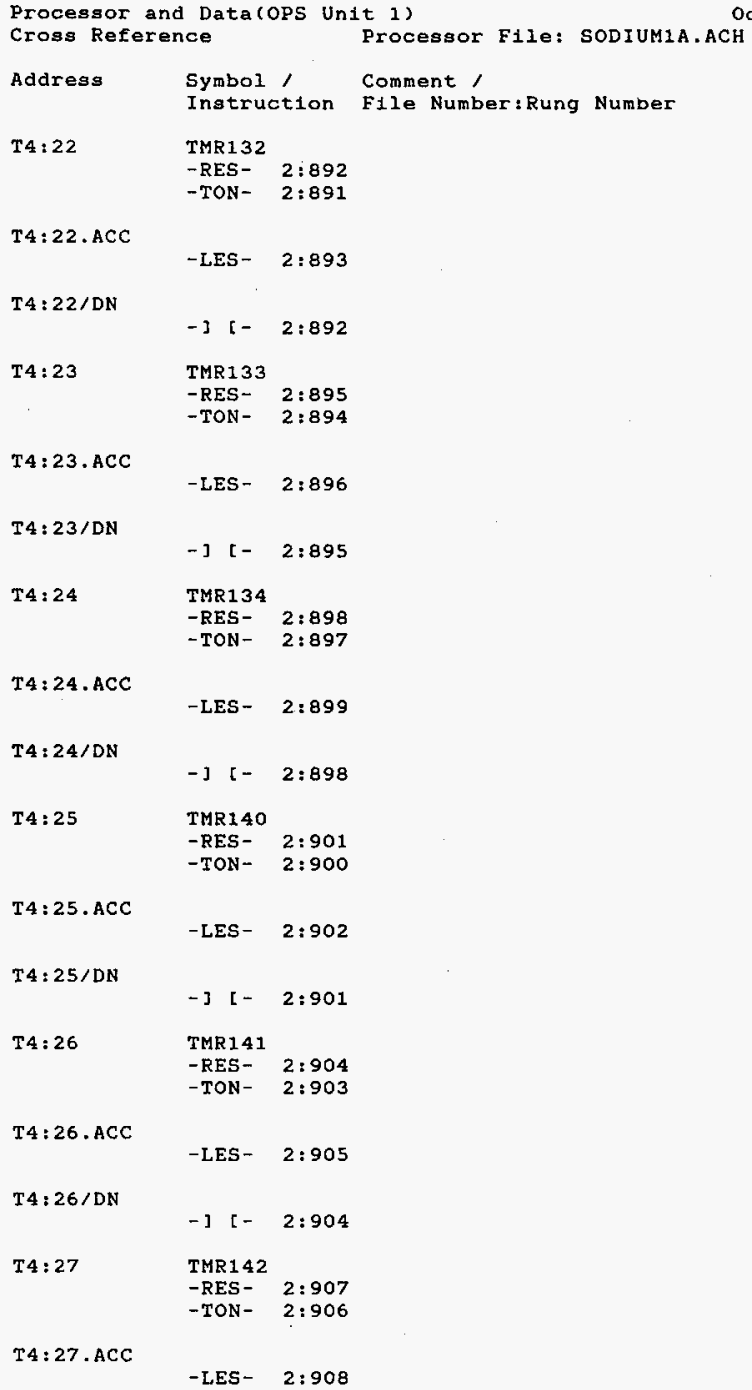

HNF-SD-FF-CSWD-60Rev. 0 
Processor and Data(OPS Unit 1)

Crosg Reference

Address

Symbol,

Processor File: SODIUMiA.ACH

Ad

Instruction

Comment /

T4:27/DN

- ] $[-\quad 2: 907$

T4:60

TMR_SU

- TON- $2: 2$

T4:60/DN

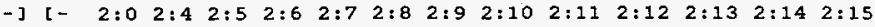

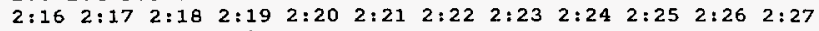
$\begin{array}{lll}2: 28 & 2: 29 \quad 2: 30 \quad 2: 31\end{array}$

$-] /[-2: 3$

$T 4: 61$

TMR_SCH

-RES- $2: 34$

- TON- $2: 33$

T4:61. ACC

-GEQ- $2: 35 \quad 2: 36 \quad 2: 37 \quad 2: 38$

$\mathrm{T} 4: 61 / \mathrm{DN}$

$-][-\quad 2: 34$

$T 4: 62$

RT TMR

-RES - 2:34

-TON- $2: 33$

T4:62/DN

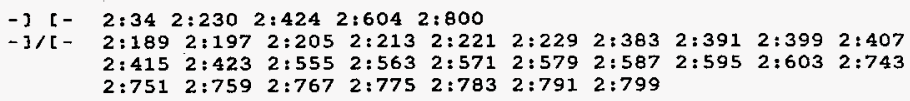

HNF-SD-FF-CSWD-60Rev. 0 
Processor and Data(ops Unit 1)

symbol Instruction

Comment,

File Number:Rung Number

$2:$ LBL 1

$$
\begin{array}{lll} 
& \text { MD1 } \\
-J M P- & 2: 3 \quad 2: 44
\end{array}
$$

$-L B L-2: 49$

$2: L B L 10$

-JMP - $2: 670$

- LBL- 2:604

2 : LBL 11

- JMP- $2: 868$

-LBL- 2:802

2 : LBL 12

-JMP- 2:131

-LBL- 2:168

2: $\operatorname{LBL} 13$

$$
\text { -JMP- 2:361 }
$$$$
\text { -LBL- } 2: 400
$$

2: LBL14

$$
\text { -JMP- 2:603 }
$$$$
\text { -LBL- 2:633 }
$$

2: L LBL 15

- JMP- 2:801

-LBL- 2:831

2: LBL 16

-JMP - 2:214

-LBL - 2:216

$2:$ LBI, 17

- JMP - 2:222

-LBL- 2:224

$2: L B L 18$

-JMP - 2:230

-LBL- $2: 232$

2:LBL19

-JMP- 2:238

-LBL- $\quad 2: 240$

$2:$ LBL2

-JMP- 2:45

-LBL- 2:295

2: LBL 20

-JMP- $2: 246$

-LBL- $2: 248$

2: LBL 21
DEV 3

DEV 4

A V 1

Av 2

AV 4

PID 143

PID144

PID150

PIDI51

MD2

PID 152
HNF-SD-FF-CSWD-60Rev. 0

Page 101

$$
\begin{array}{ll} 
& \text { PID153 } \\
\text {-JMP- } 2: 254 \\
\text { - LBL- } 2: 256
\end{array}
$$




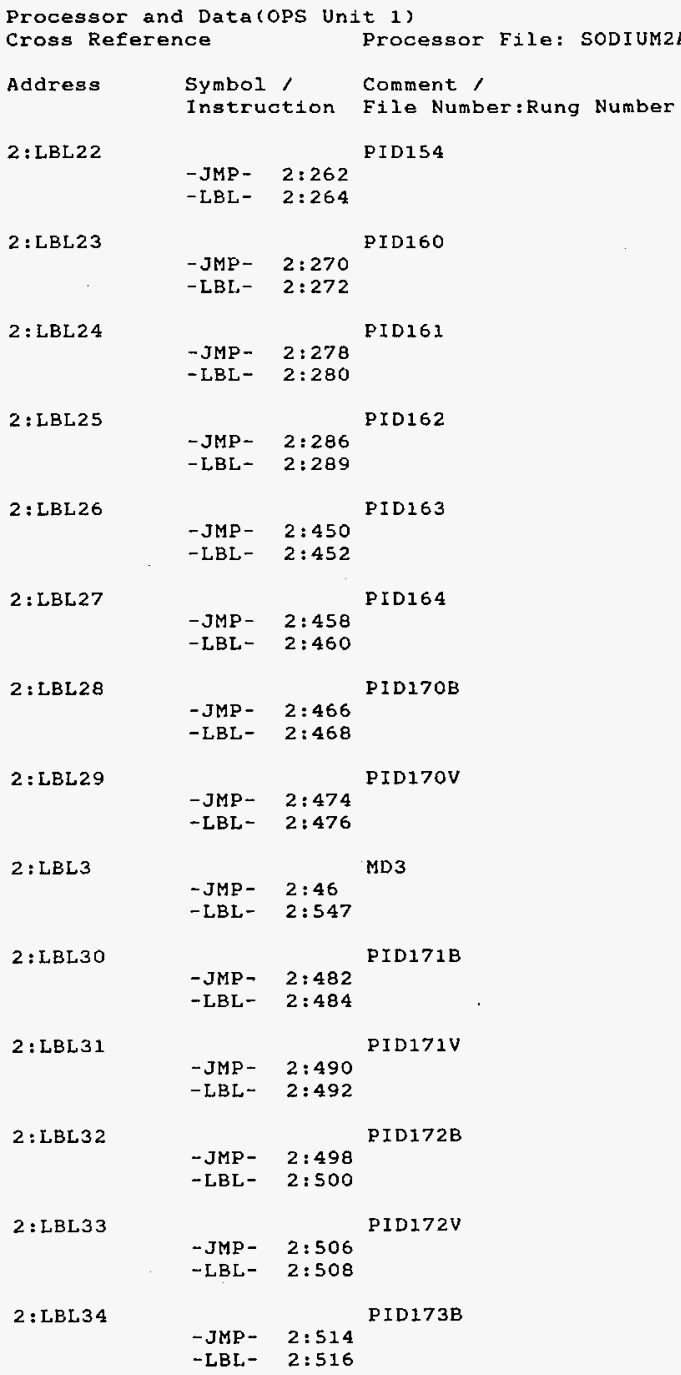

PIDI70B

-LBL- 2:516

PID16I

PID162

PID163

PID164

PID170V

MD3

PID171B

PID171V

PID172B

PID172V

PID173B

HNF-SD-FF-CSWD-60Rev. 0 
Processor and Data(OPS Unit 1)

\section{Cross Reference}

Address

2 : LBL 35

$2: \operatorname{LBL} 36$

2: LBL 39

$2:$ LBL4

$2:$ LBL 40

$2: \operatorname{LBL} 43$

$2: \operatorname{LBL} 44$

$2:$ LBL 45

$2:$ LBL 47
Processor File: SODIUM2A.ACH

October 23, 1996

Page 3 Comment,

File Number:Rung Number

PID $173 \mathrm{~V}$

- JMP- $2: 522$

$-\mathrm{LBL}-\quad 2: 524$

- JMP - 2:530

PID174B

$-\mathrm{LBL}-\quad 2: 532$

PID 17AV

-JMP- $2: 538$

-LBL- $2: 541$

PID175B

-JMP- $2: 672$

$-L B L-\quad 2: 674$

-JMP - $2: 680$

-LBL- $2: 682$

-LBL- $2: 745$

- JMP- 2:688

-LBL- 2:690

-JMP- 2:696

-LBL- 2:698

PID176V

PID176B

PID $175 \mathrm{~V}$

MD4

HNF-SD-FF-CSWD-6ORev. 0

Page 103

JMP- $2: 704$

-LBL- 2:706

PID177B

PID177V

- JMP- $2: 712$

-LBL- $2: 714$

-JMP - $2: 720$

$-I B L-\quad 2: 722$

PID178B

-JMP- $2: 728$

-LBL- $2: 730$

PID178V

PID179B

-JMP- $\quad 2: 736$

-LBL- 2:739

- JMP- $2: 870$

PID179V

-LBL- 2:872 


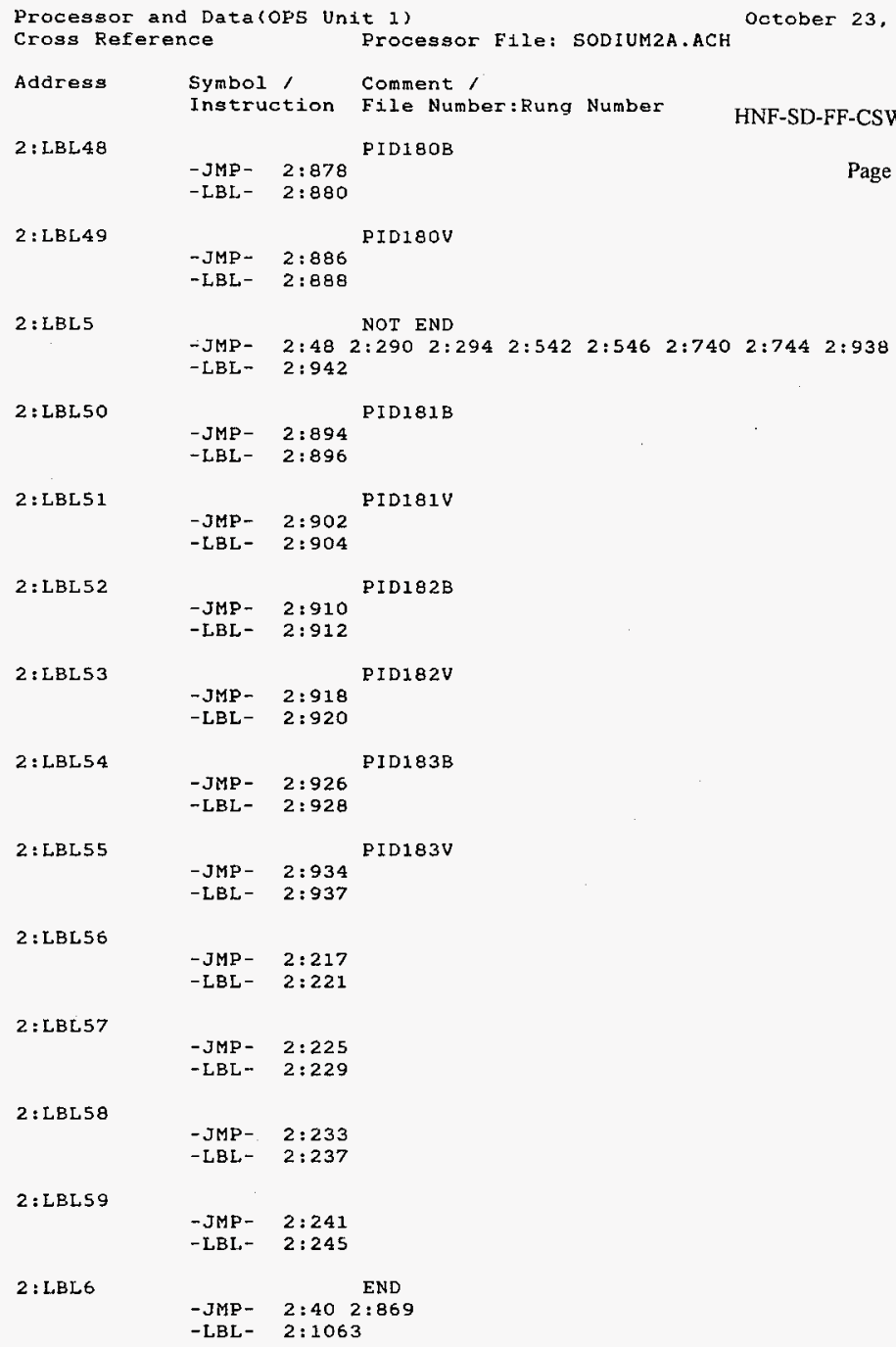




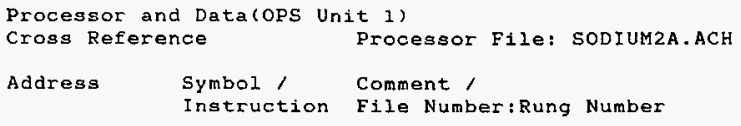

2:LBL60

-JMP- $\quad 2: 249$

$-\mathrm{LBL}-\quad 2: 253$

HNF-SD-FF-CSWD-6DRev. 0

$2:$ LBL61

-JMP - 2:257

-LBL- 2:261

Page 105

2: LBL62

-JMP- 2:265

-LBL- 2:269

$2:$ LBL63

-JME- $2: 273$

-LBL- 2:277

$2: \operatorname{LBL} 64$

- JMP - 2:281

-LBL- $\quad 2: 285$

2:LBL66

-JMP- $2: 453$

-LBL- $2: 457$

2 : LBL67

- JMP - 2:46I

-LBL- $2: 465$

2 : LBL 68

-JMP- 2:469

-LBL- $2: 473$

2: LBL69

- JMP- $2: 477$

-LBL - 2:481

2:LBL7

- JMP - $2: 0$

$-\mathrm{LBL}-\quad 2: 41$

2: LBL.70

- JMP- $2: 485$

-LBL- $2: 489$

$2: \operatorname{LBL} 71$

-JMP- $2: 493$

-LBL- 2:497

2: LBL72

- JMP- 2:501

-LBL- 2:505

$2: \operatorname{LBL} 73$

-JMP - 2:509

-LBL - 2:513 


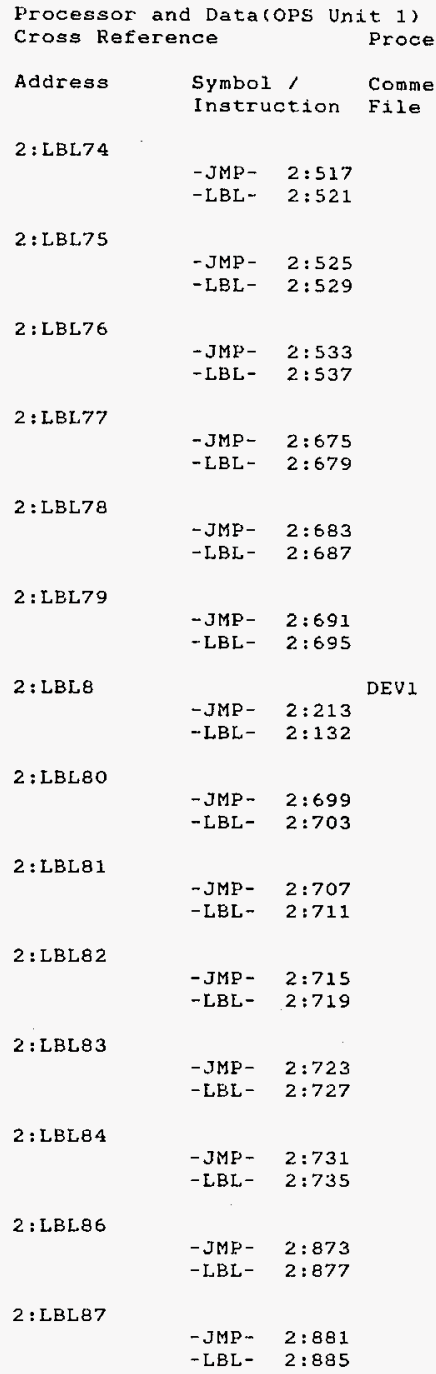

HNF-SD-FF-CSWD-60Rev. 0

Page 106 
Processor and Datacops Unit 1 )

October 23, 1996 Page 7

Addregs

Symbol , Instruction

Comment

File Number:Rung Number

2. $\mathrm{LBL} 88$

$$
\begin{array}{ll}
\text {-JMP- } & 2: 889 \\
\text {-LBL- } & 2: 893
\end{array}
$$

2:LBL89

$$
\begin{array}{ll}
\text {-JMP- } & 2: 897 \\
-L B L- & 2: 901
\end{array}
$$

2: LBL9

$$
\text { -JMP- } 2: 449
$$$$
-\mathrm{LBL}-\quad 2: 362
$$

2: LBL 90

$$
\text { - JMP- 2:905 }
$$$$
-\mathrm{LBL}-\quad 2: 909
$$

2: LBL9 1

$$
\text { - JMP - 2:913 }
$$$$
\text { -LBL- } 2: 917
$$

$2: L B L 92$

$$
\text { - JMP- } \quad 2: 921
$$$$
\text { -LBL - 2:925 }
$$

$2: \operatorname{LBL} 93$

$$
\begin{array}{ll}
\text { - JMP - } & 2: 929 \\
\text {-LBL- } & 2: 933
\end{array}
$$

3

F8:0

$$
\text { -JSR- 2:1 }
$$

TOTAL

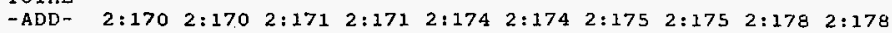

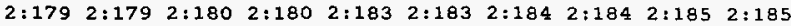

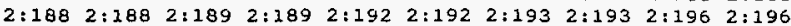

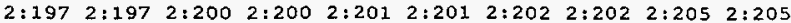

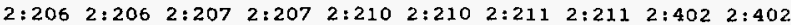

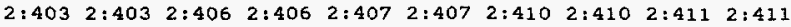

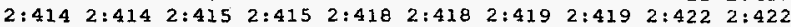

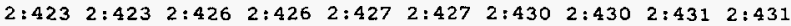

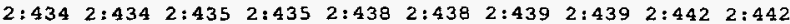

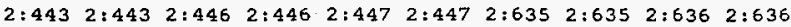

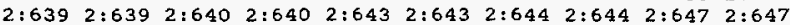
$\begin{array}{llllllllll}2: 648 & 2: 648 & 2: 651 & 2: 651 & 2: 652 & 2: 652 & 2: 655 & 2: 655 & 2: 656 & 2: 656\end{array}$ $\begin{array}{lllllllllll}2: 659 & 2: 659 & 2: 660 & 2: 660 & 2: 663 & 2: 663 & 2: 664 & 2: 664 & 2: 667 & 2: 667\end{array}$ $\begin{array}{llllllllll}2: 658 & 2: 668 & 2: 833 & 2: 833 & 2: 834 & 2: 834 & 2: 837 & 2: 837 & 2: 838 & 2: 838\end{array}$

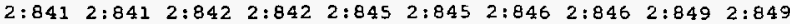

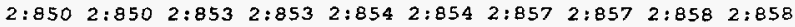
$\begin{array}{llllllll}2: 861 & 2: 861 & 2: 862 & 2: 862 & 2: 865 & 2: 865 & 2: 866 & 2: 866\end{array}$

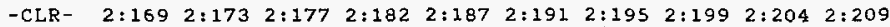

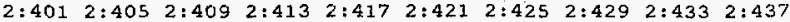

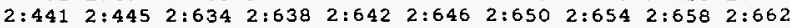
$\begin{array}{llllllllll}2: 666 & 2: 832 & 2: 836 & 2: 840 & 2: 844 & 2: 848 & 2: 852 & 2: 856 & 2: 860 & 2: 864\end{array}$

-DIV- $2: 172 \quad 2: 176 \quad 2: 181 \quad 2: 186 \quad 2: 190 \quad 2: 194 \quad 2: 198 \quad 2: 203 \quad 2: 208 \quad 2: 212$ $\begin{array}{llllllllll}2: 404 & 2: 408 & 2: 412 & 2: 416 & 2: 420 & 2: 424 & 2: 428 & 2: 432 & 2: 436 & 2: 440\end{array}$

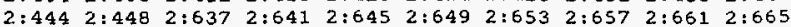

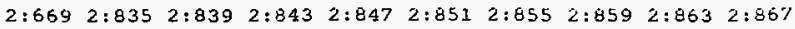




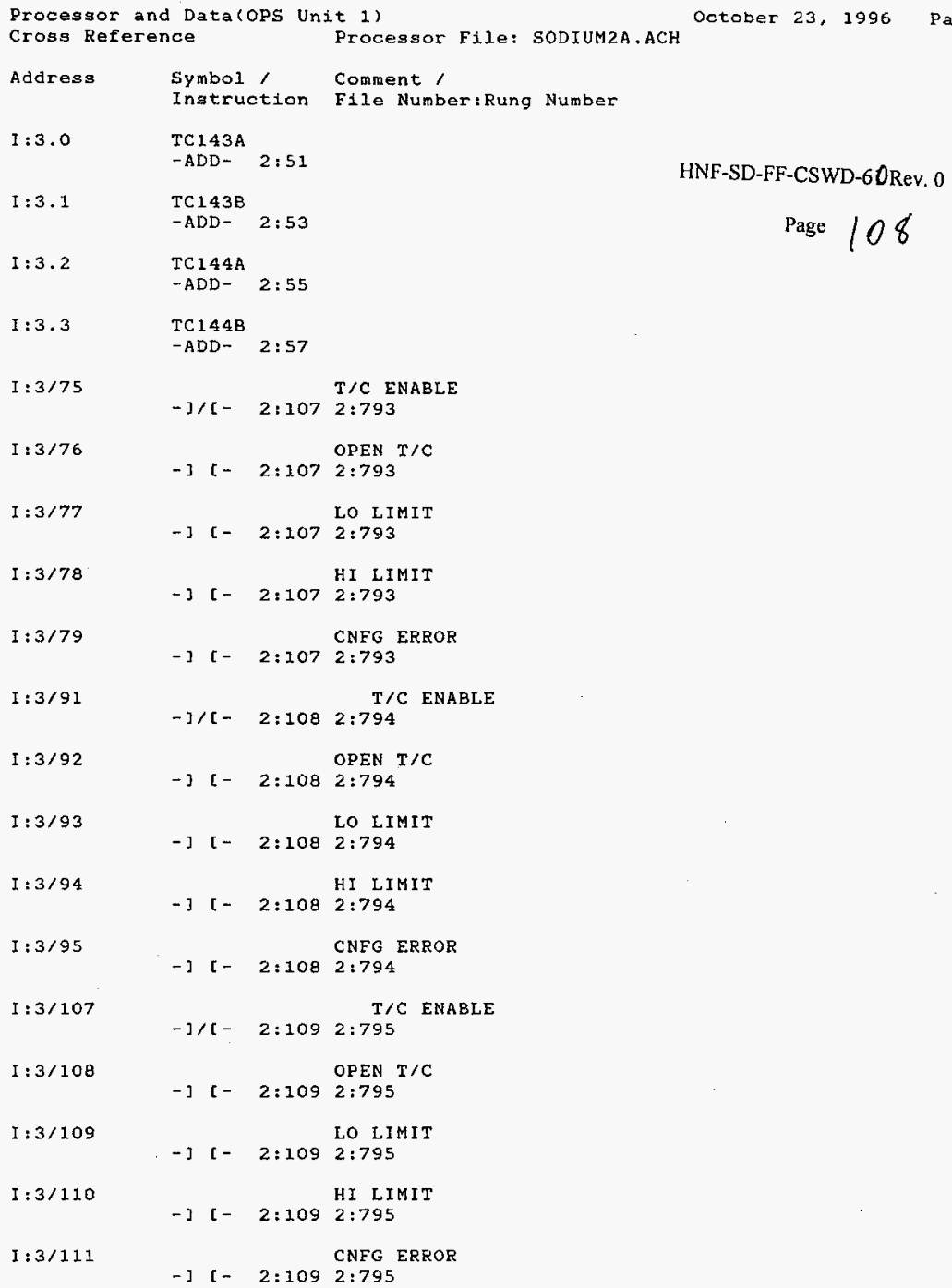

HNF-SD-FF-CSWD-60Rev. 0

Page 108 


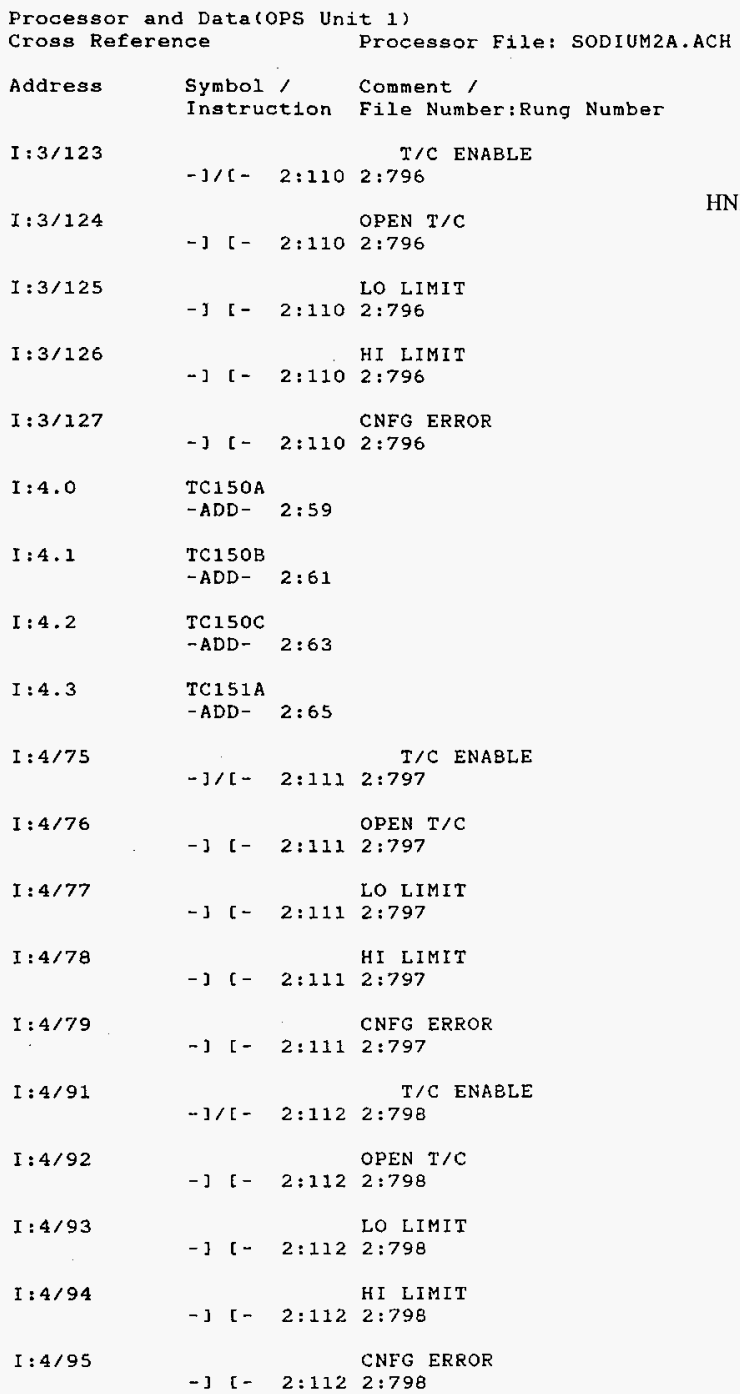




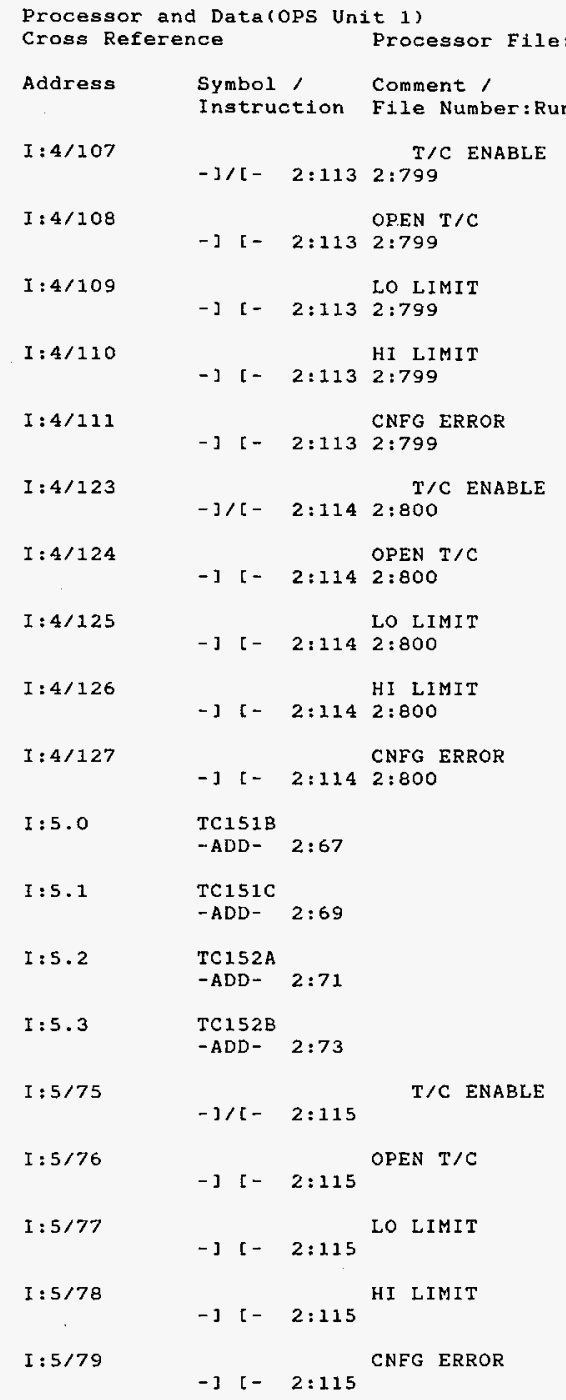




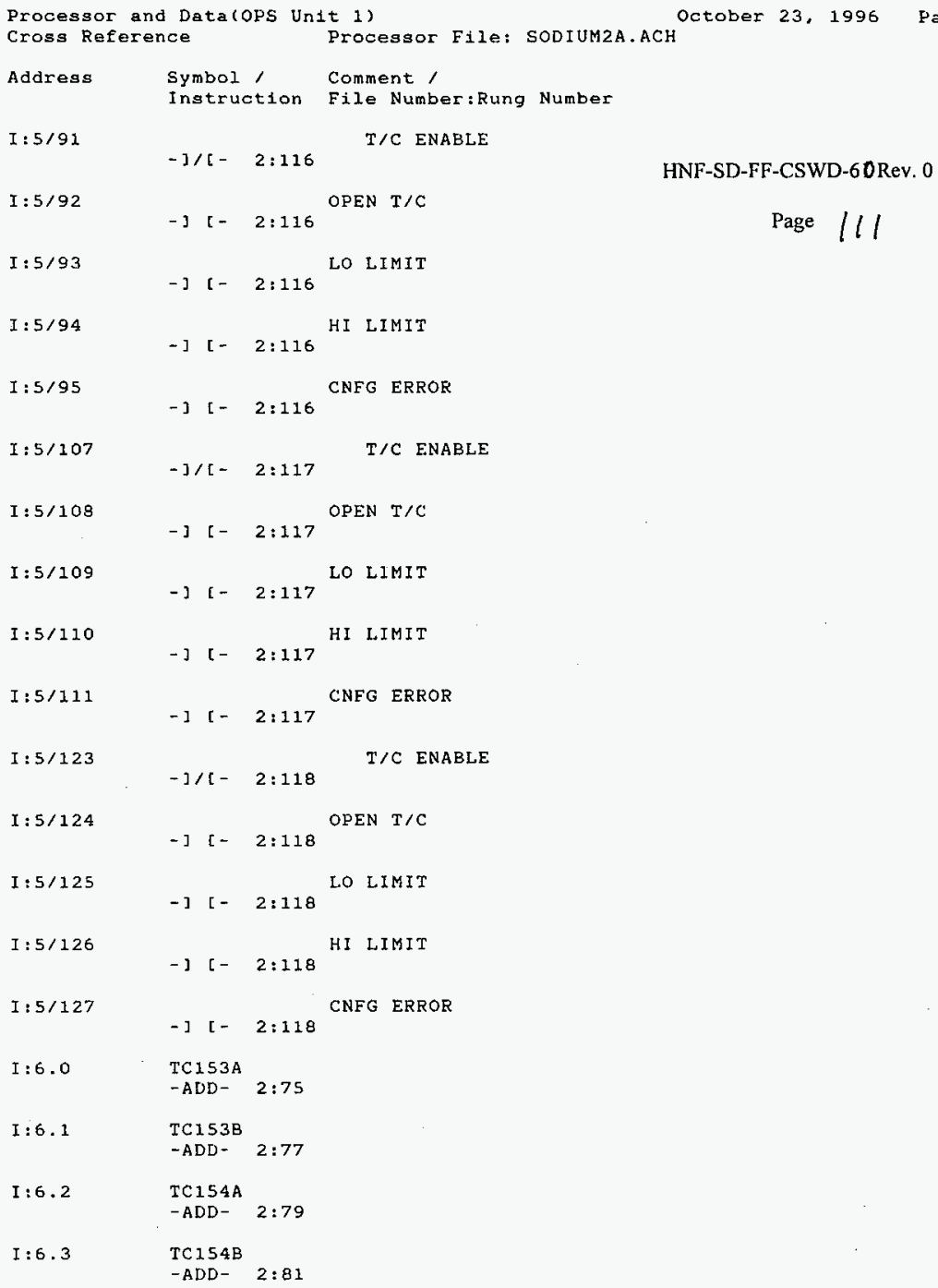

HNF-SD-FF-CSWD-60Rev. 0 


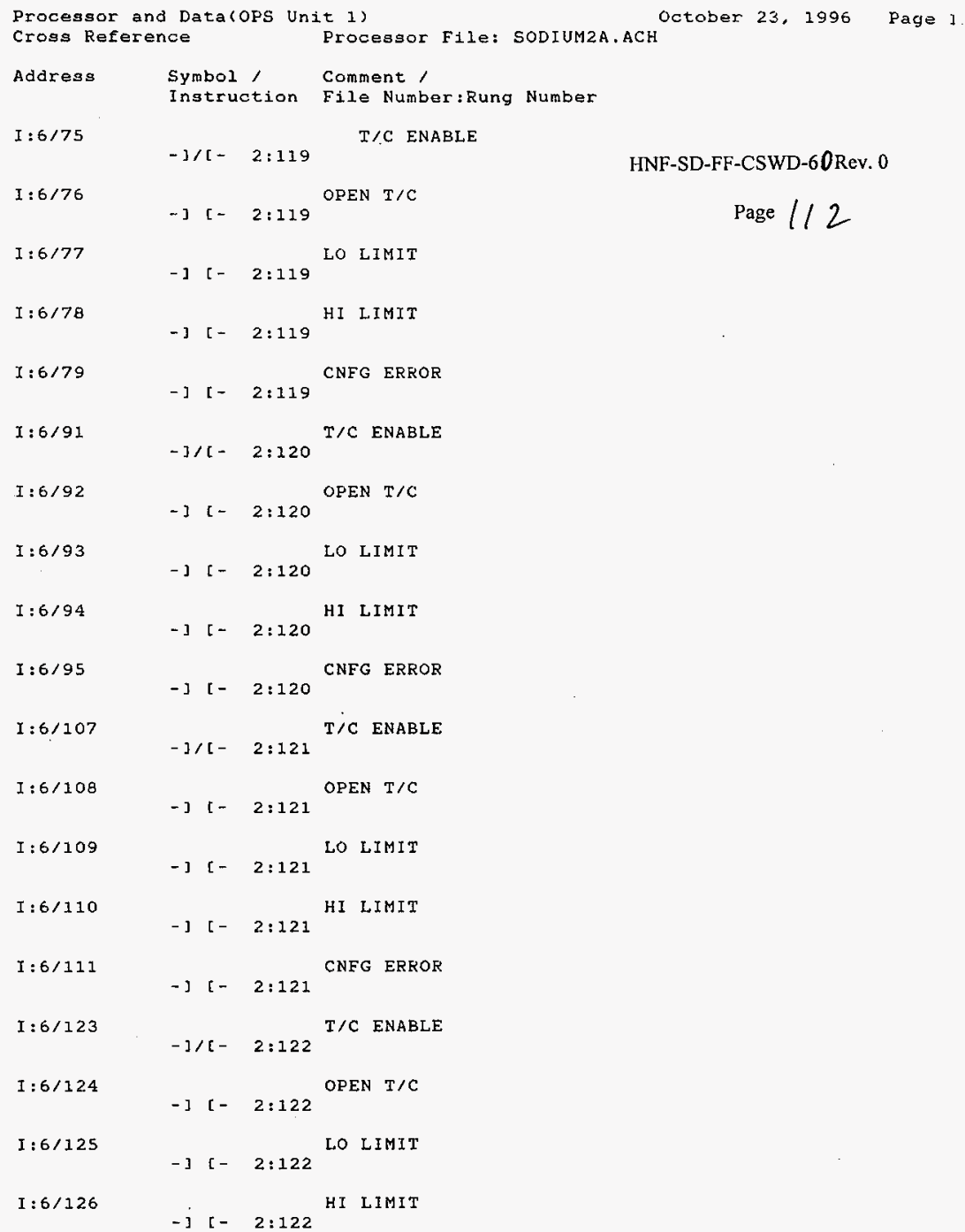

HNF-SD-FF-CSWD-60Rev. 0

Page $/ / 2$ 


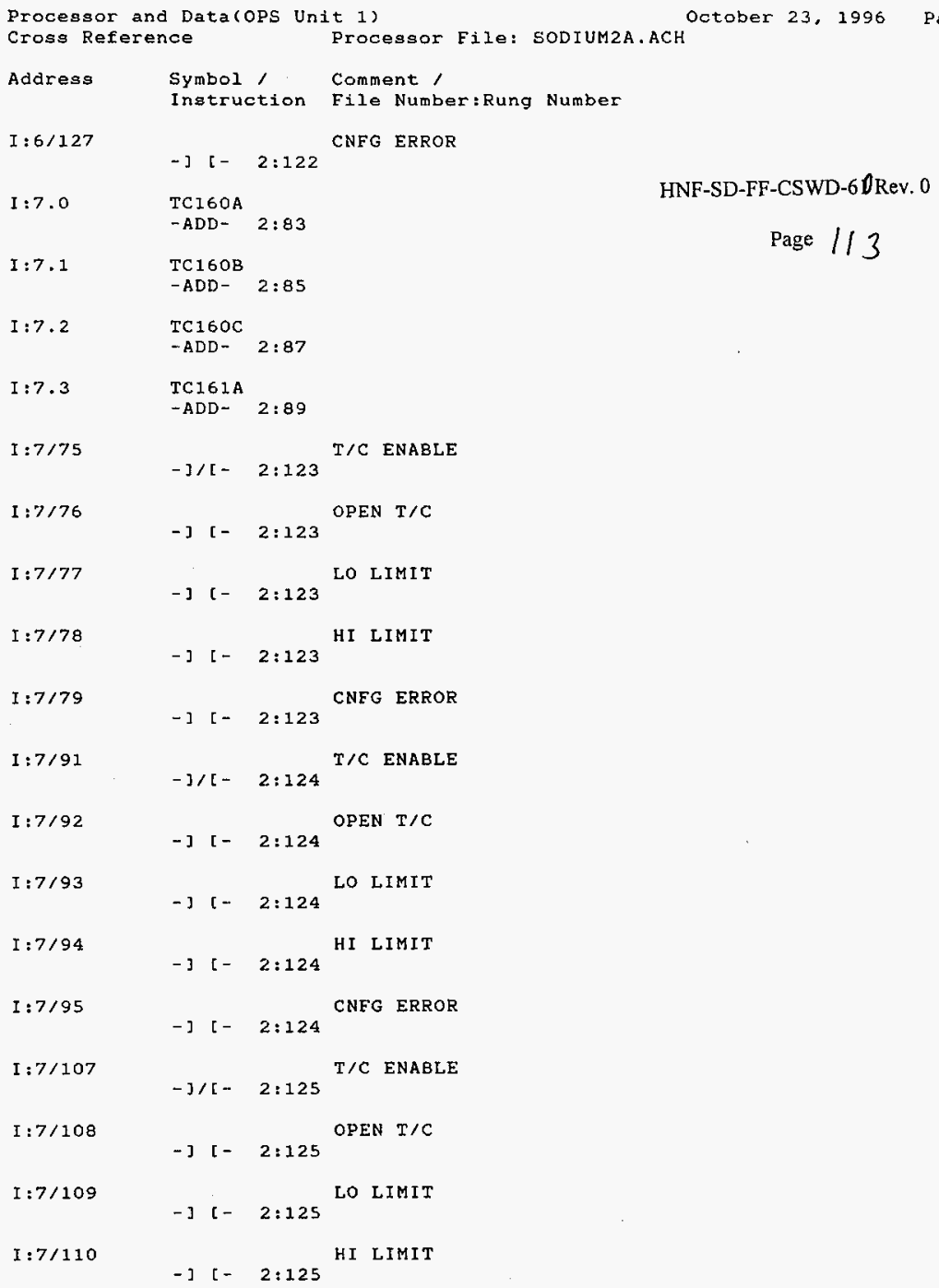

HNF-SD-FF-CSWD-60Rev. 0

Page $/ / 3$ 


\begin{tabular}{|c|c|c|c|c|}
\hline Address & $\begin{array}{l}\text { Symbol } \\
\text { Instruc }\end{array}$ & $\begin{array}{l}\prime \\
\text { ction }\end{array}$ & $\begin{array}{l}\text { Comment } \\
\text { File Number: Rung }\end{array}$ & Number \\
\hline$I: 7 / 111$ & $-][-$ & $2: 125$ & CNFG ERROR & \\
\hline$I: 7 / 123$ & $-] /[-$ & $2: 126$ & $T / C$ ENABLE & \\
\hline$I: 7 / 124$ & $-][-$ & $2: 126$ & OPEN $\mathrm{T} / \mathrm{C}$ & \\
\hline$I: 7 / 125$ & $-][-$ & $2: 126$ & LO LIMIT & \\
\hline $1: 7 / 126$ & $-] \quad[-$ & $2: 126$ & HI LIMIT & \\
\hline$I: 7 / 127$ & $-] \quad[-$ & $2: \pm 26$ & CNFG ERROR & \\
\hline$I: 8.0$ & $\begin{array}{l}\text { TC161B } \\
\text { - ADD- }\end{array}$ & $2: 91$ & & \\
\hline$I: 8.1$ & $\begin{array}{l}\text { TC161C } \\
\text {-ADD- }\end{array}$ & $2: 93$ & & \\
\hline $1: 8.2$ & $\begin{array}{l}\text { TC162A } \\
-A D D-\end{array}$ & $2: 95$ & & \\
\hline$I: 8.3$ & $\begin{array}{l}\text { TC162B } \\
\text {-ADD- }\end{array}$ & $2: 97$ & & \\
\hline$I: 8 / 75$ & $-] /[-$ & $2: 127$ & $T / C$ ENABLE & \\
\hline$I: 8 / 76$ & $-][-$ & $2: 127$ & OPEN $\mathrm{T} / \mathrm{C}$ & \\
\hline$I: 8 / 77$ & $-]$ [ - & $2: 127$ & LO LIMIT & \\
\hline$I: 8 / 78$ & - ] [ - & $2: 127$ & HI LIMIT & \\
\hline$I: 8 / 79$ & - ] [- & $2: 127$ & CNFG ERROR & \\
\hline$I: 8 / 91$ & $-] /[-$ & $2: 128$ & $\begin{array}{l}\text { T/C ENABLE } \\
2: 789\end{array}$ & \\
\hline I $: 8 / 92$ & $-][-$ & $2: 128$ & $\begin{array}{l}\text { OPEN T/C } \\
2: 789\end{array}$ & \\
\hline$I: 8 / 93$ & $-][-$ & $2: 128$ & $\begin{array}{l}\text { LO LIMIT } \\
2: 789\end{array}$ & \\
\hline I : $8 / 94$ & $-] t-$ & $2: 128$ & $\begin{array}{l}\text { HI LIMIT } \\
2: 789\end{array}$ & \\
\hline
\end{tabular}

HNF-SD-FF-CSWD-6ORev. 0

Page 114 


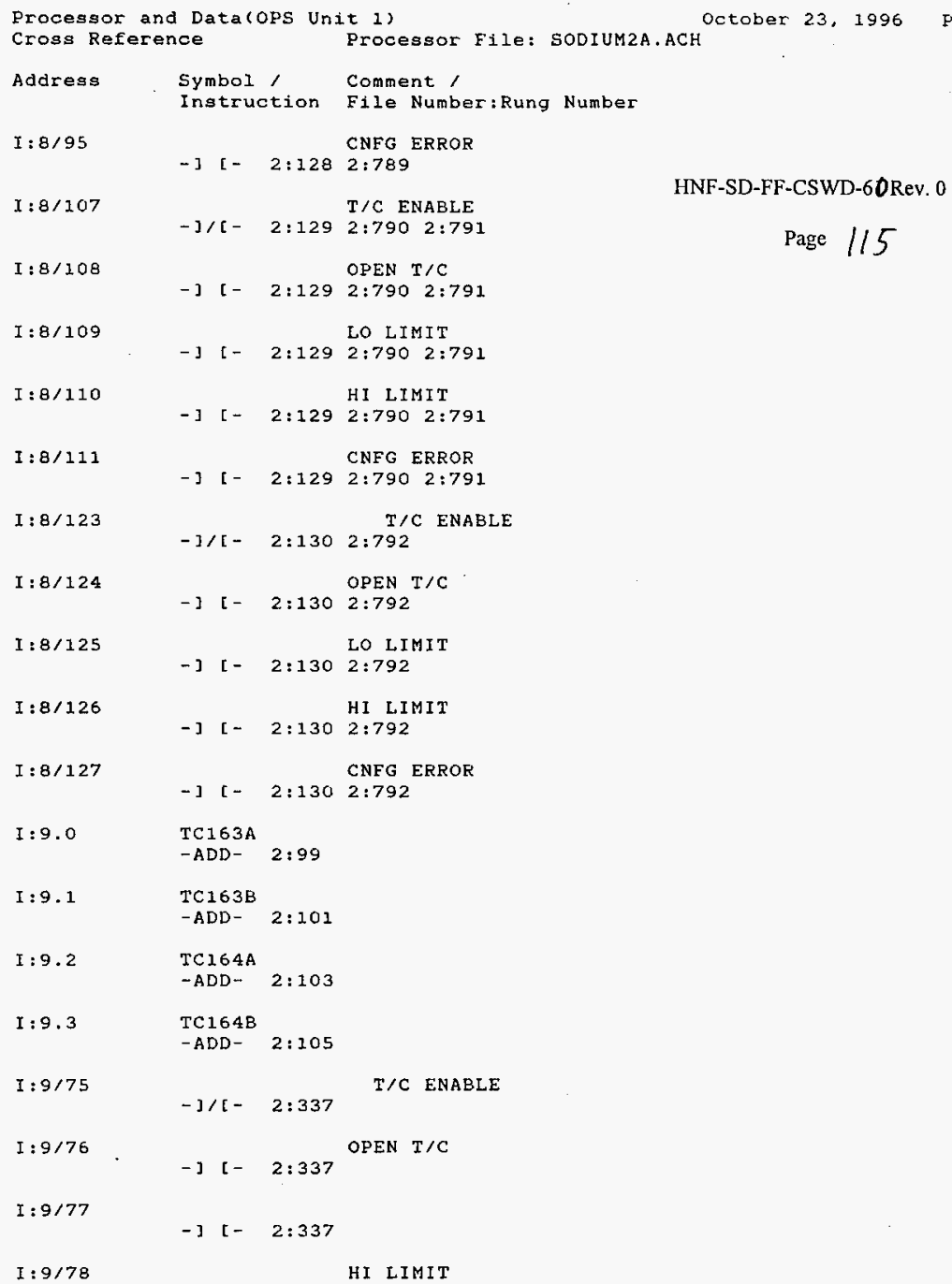

HNF-SD-FF-CSWD-6DRev. 0

$$
\text { Page } 1 / 5
$$




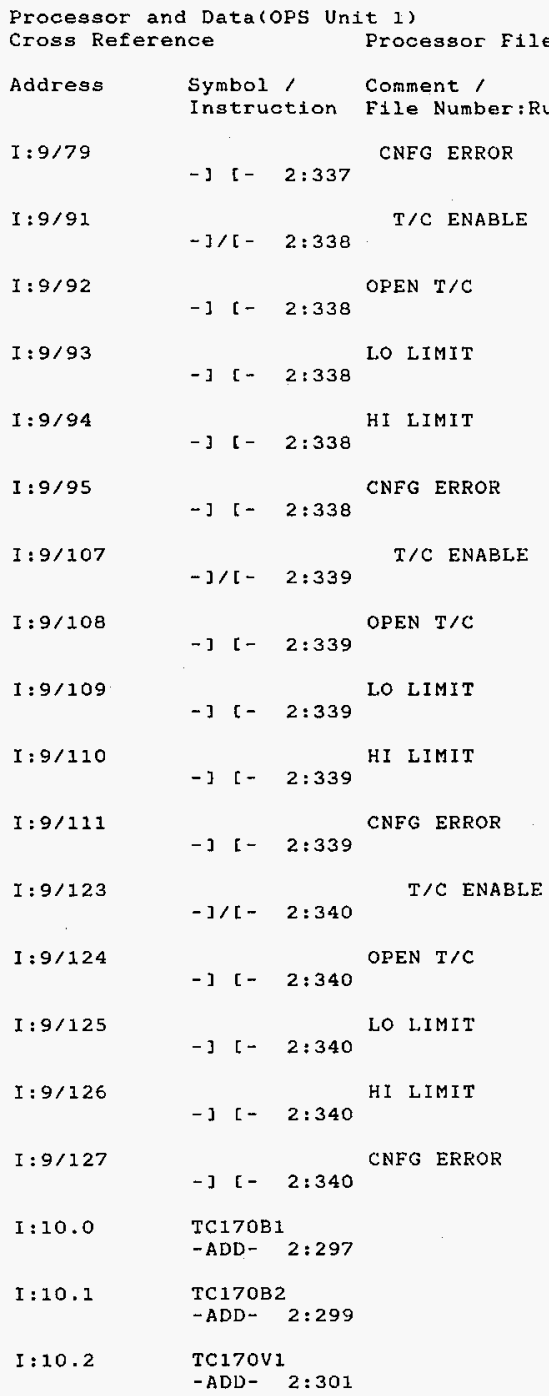

HNF-SD-FF-CSWD-60Rev. 0

Page 116 


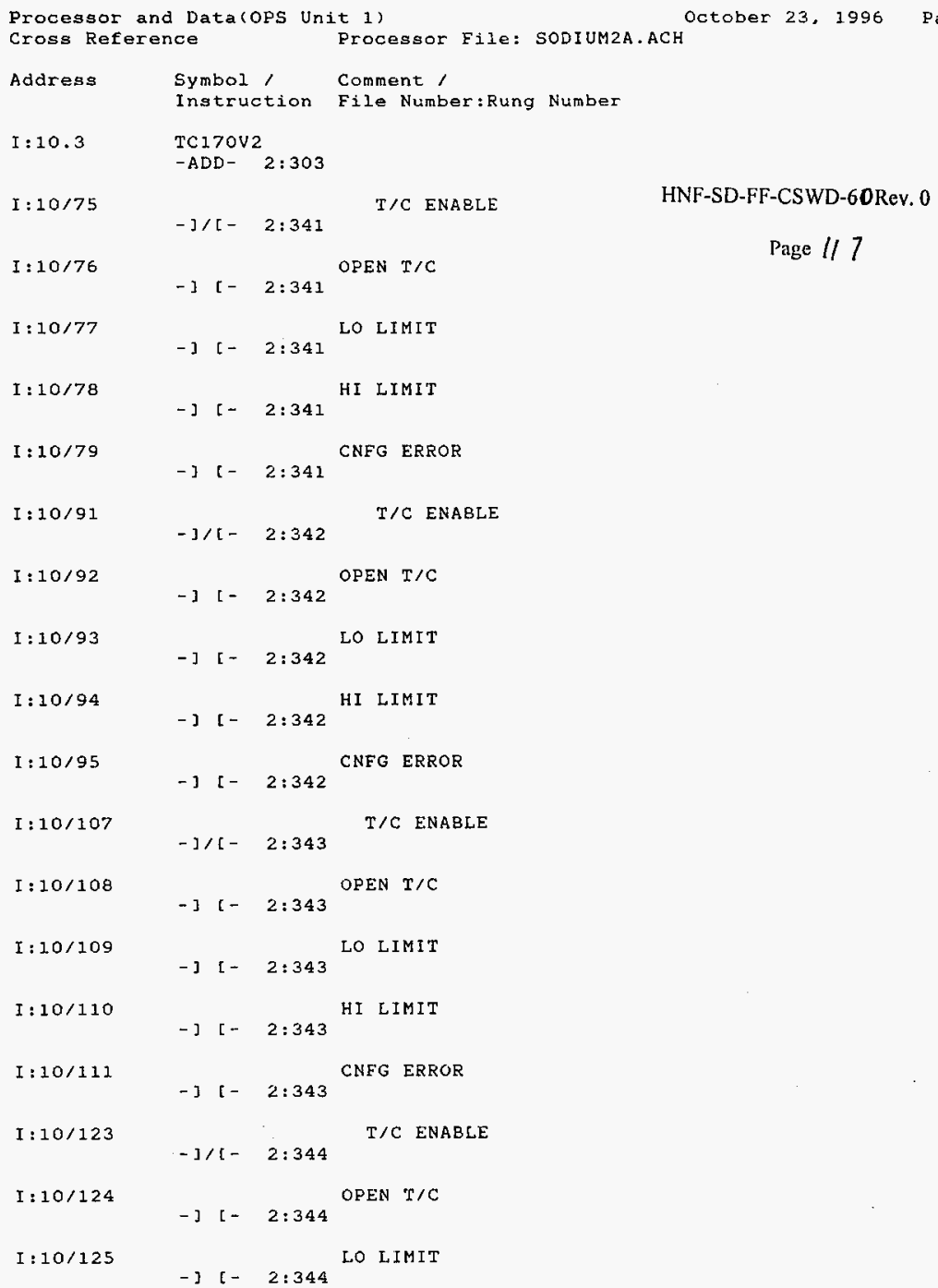




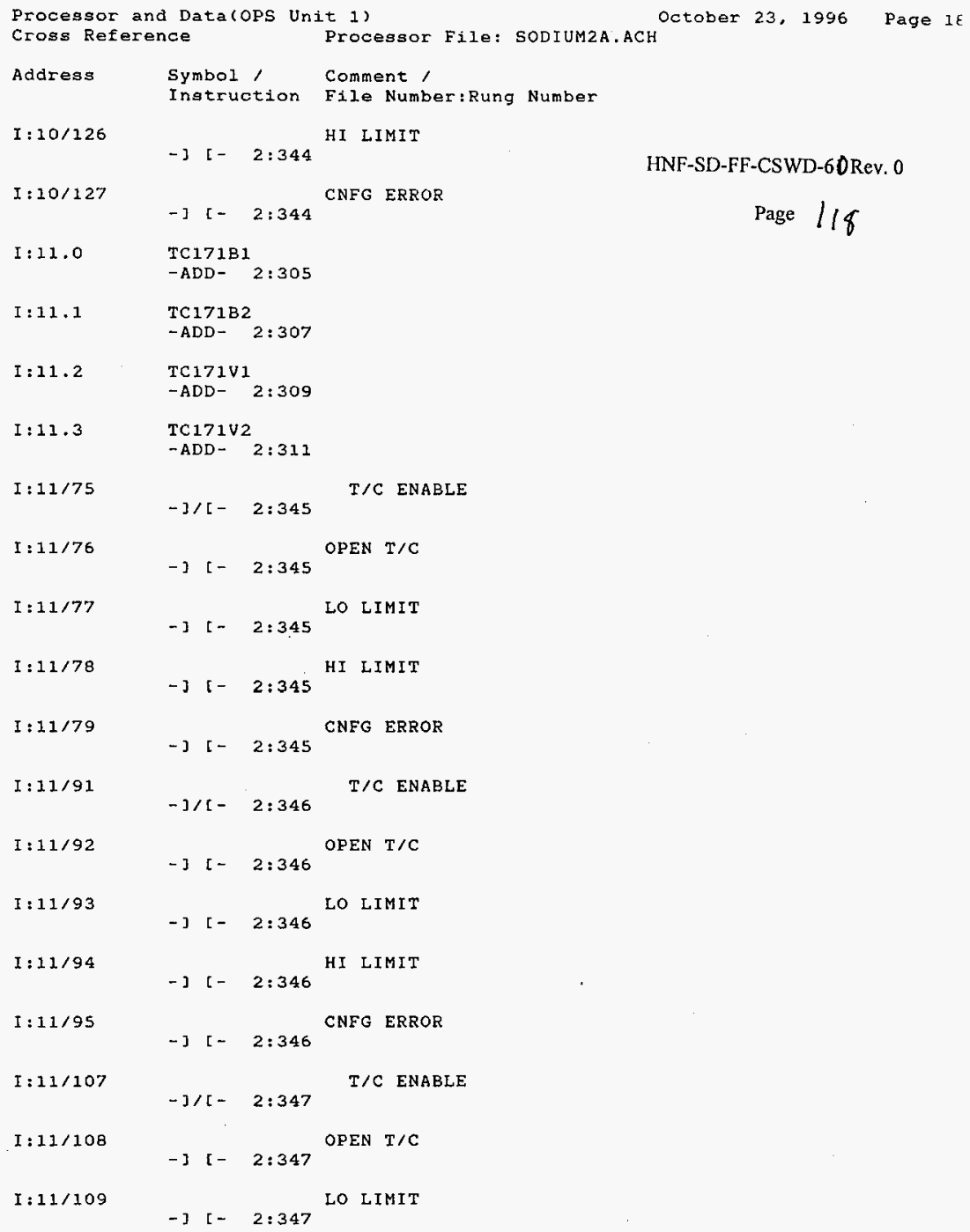

HNF-SD-FF-CSWD-60Rev. 0

Page $1 / 8$ 


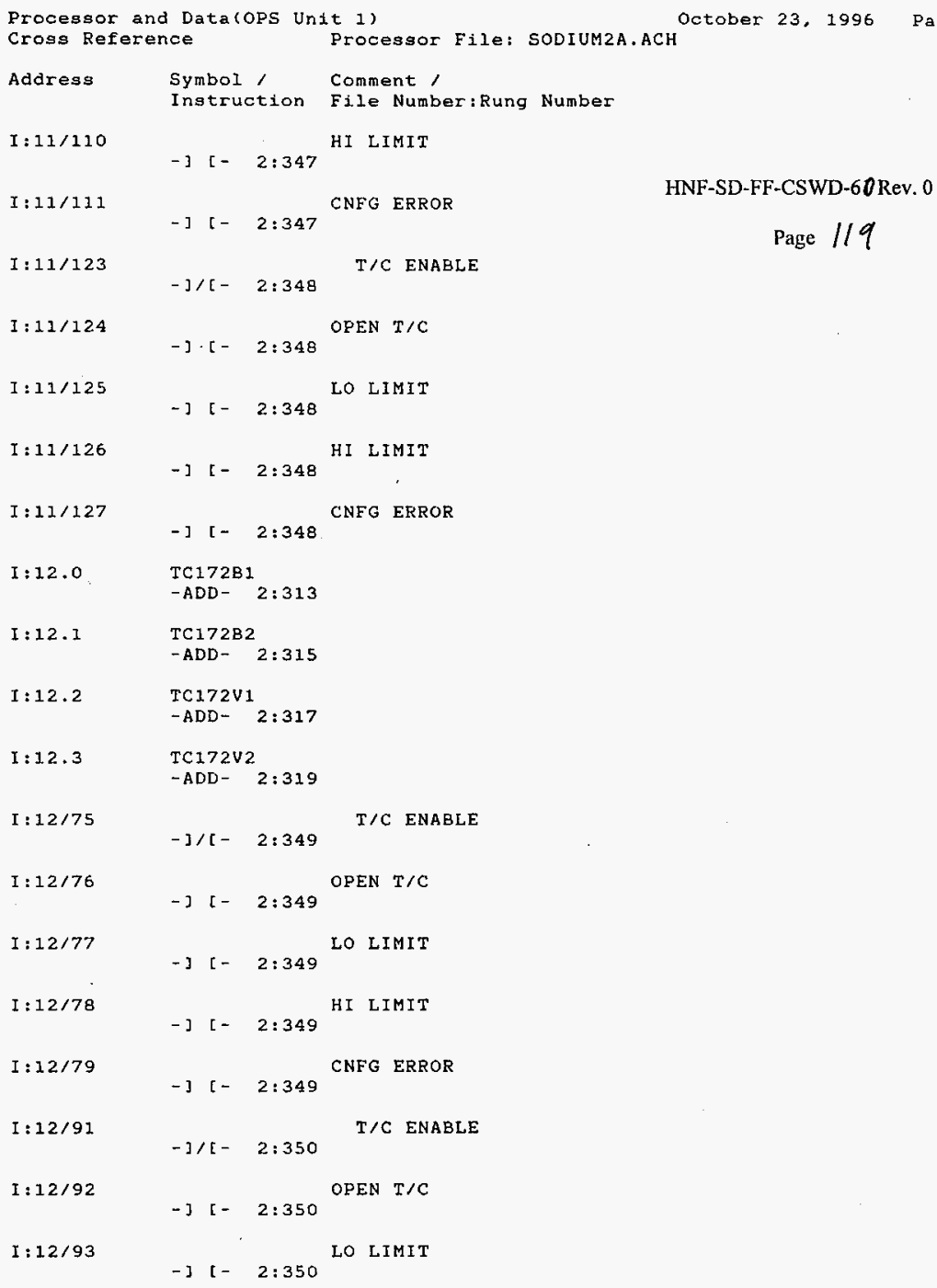




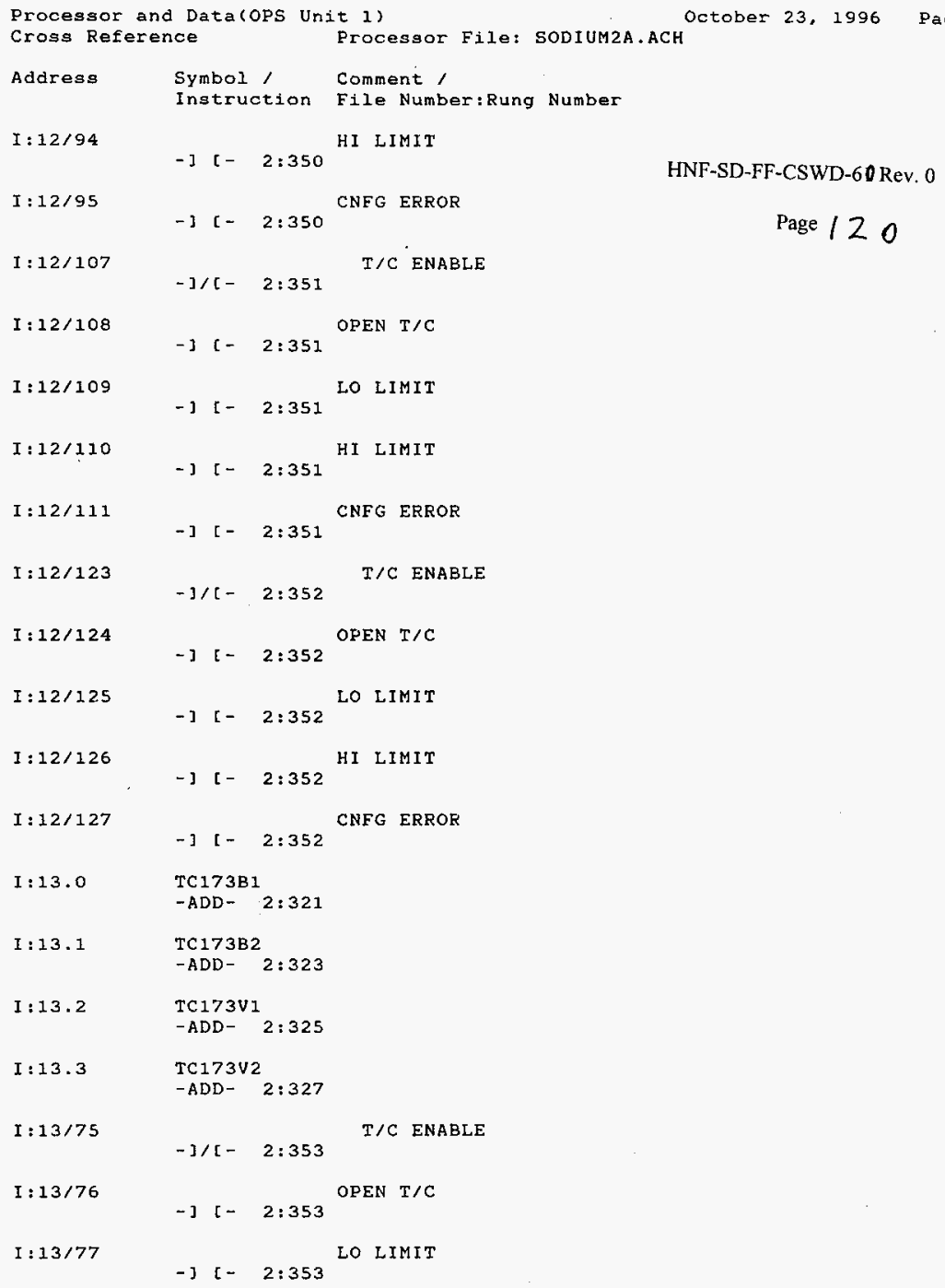

HNF-SD-FF-CSWD-60 Rev. 0 


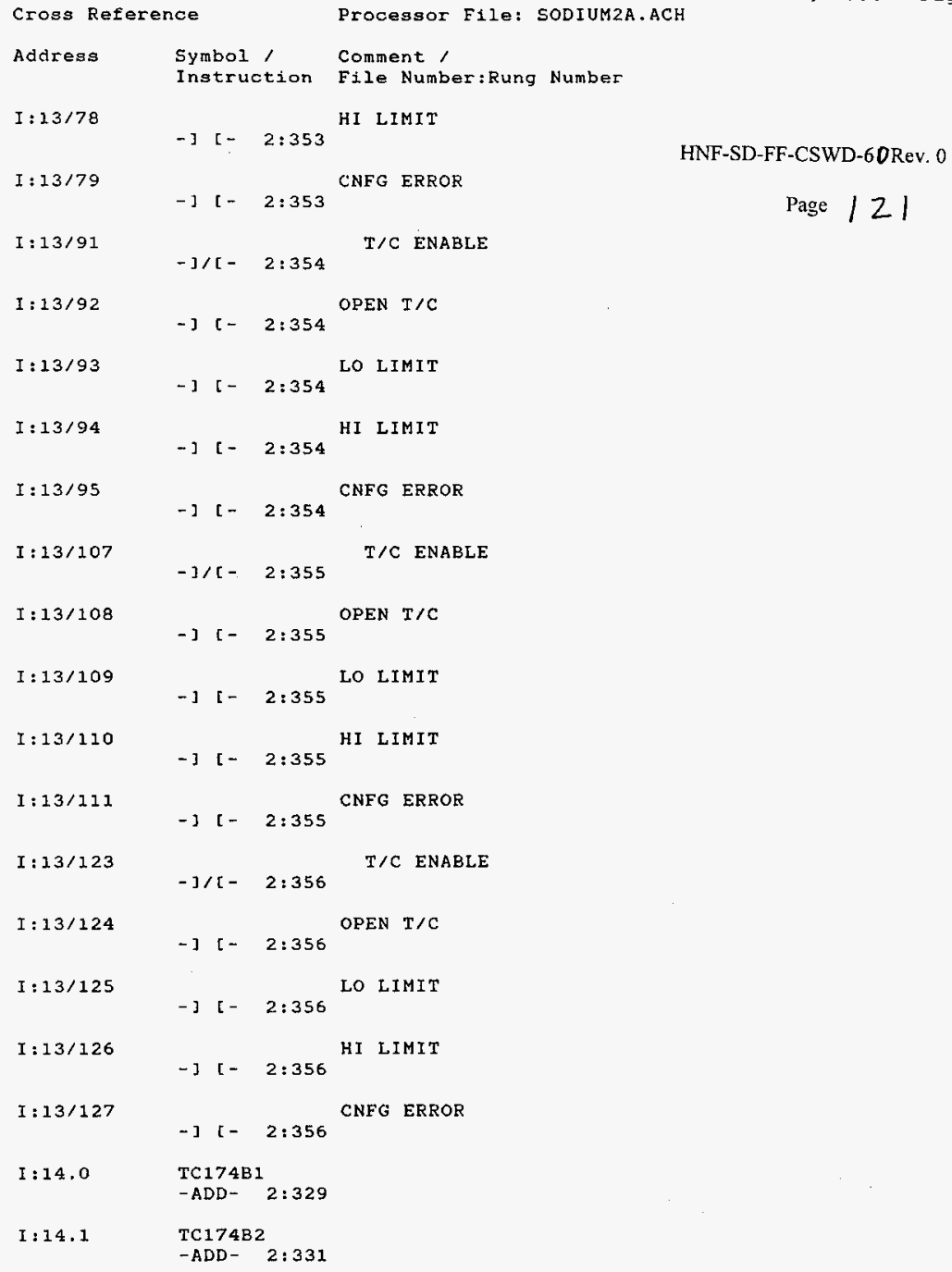

HNF-SD-FF-CSWD-60Rev. 0

Page $|2|$ 


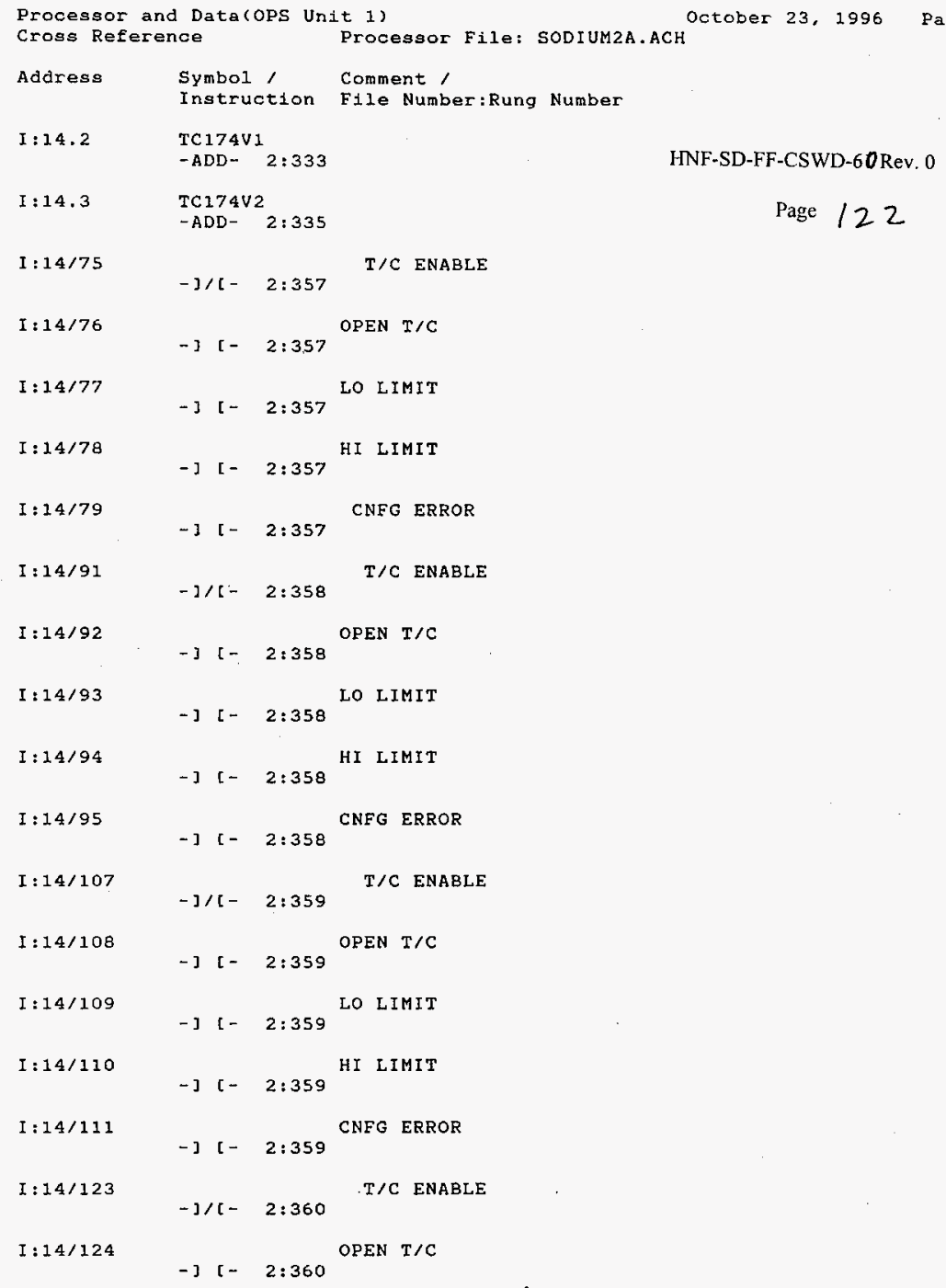




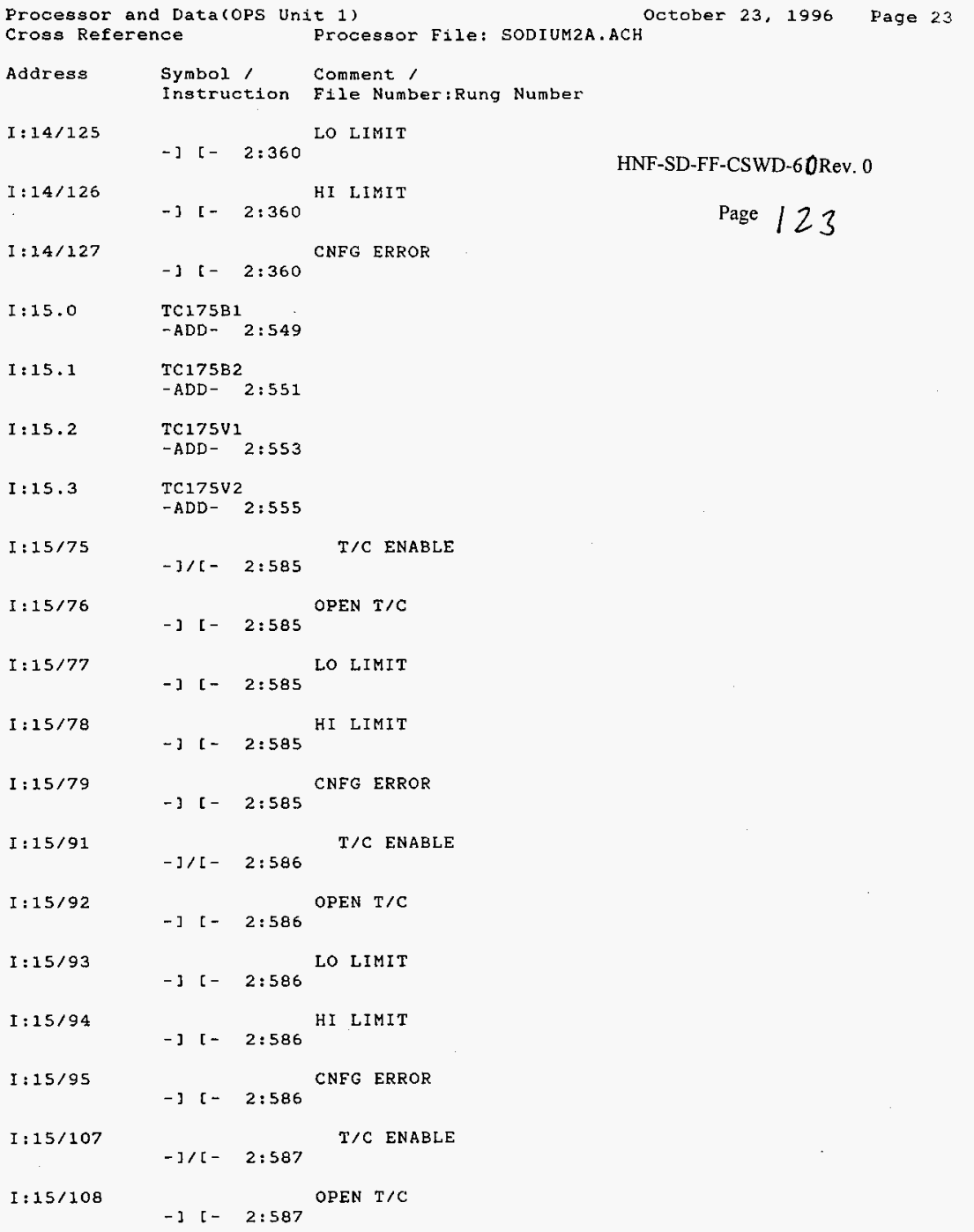

HNF-SD-FF-CSWD-60Rev. 0

Page 123 


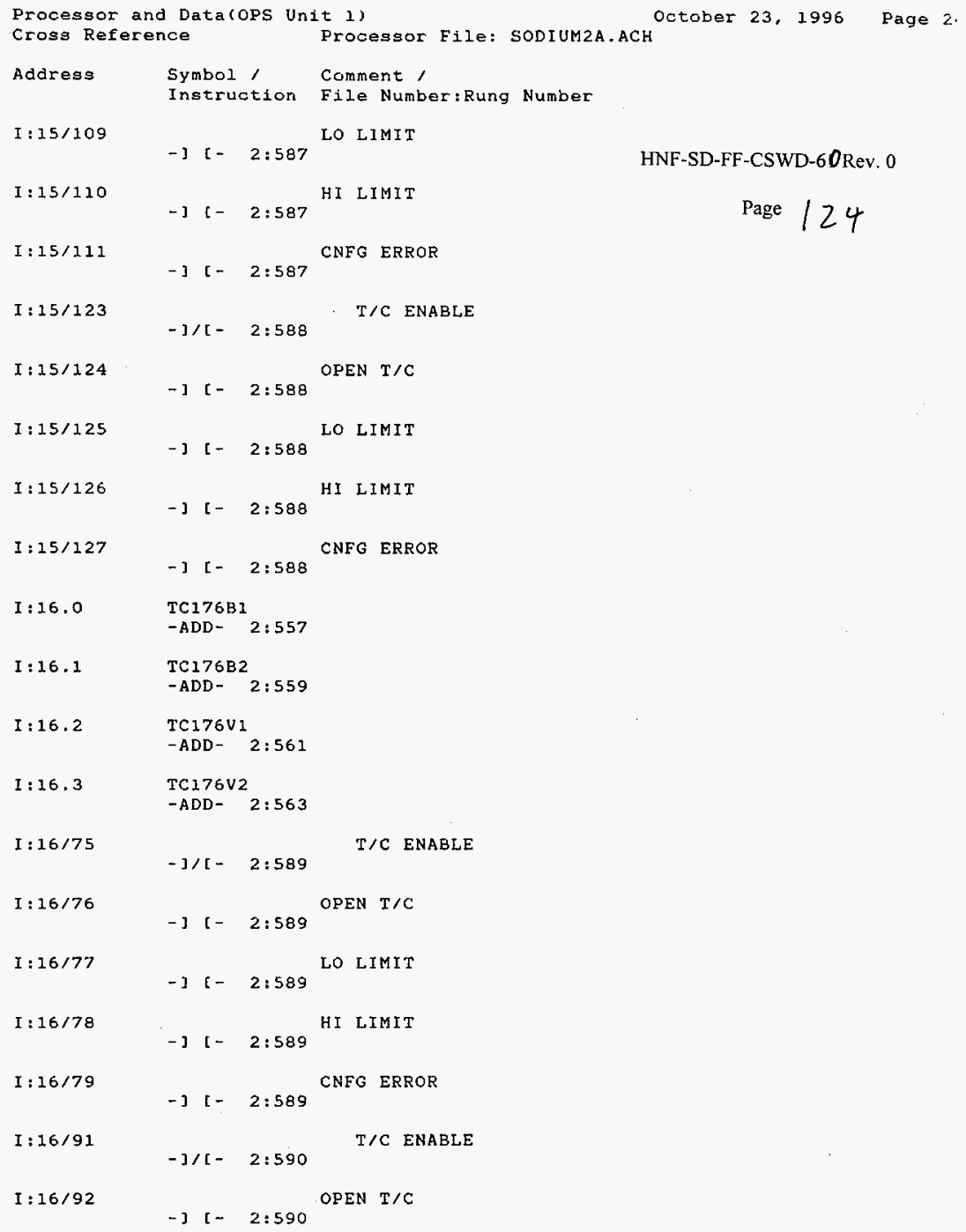




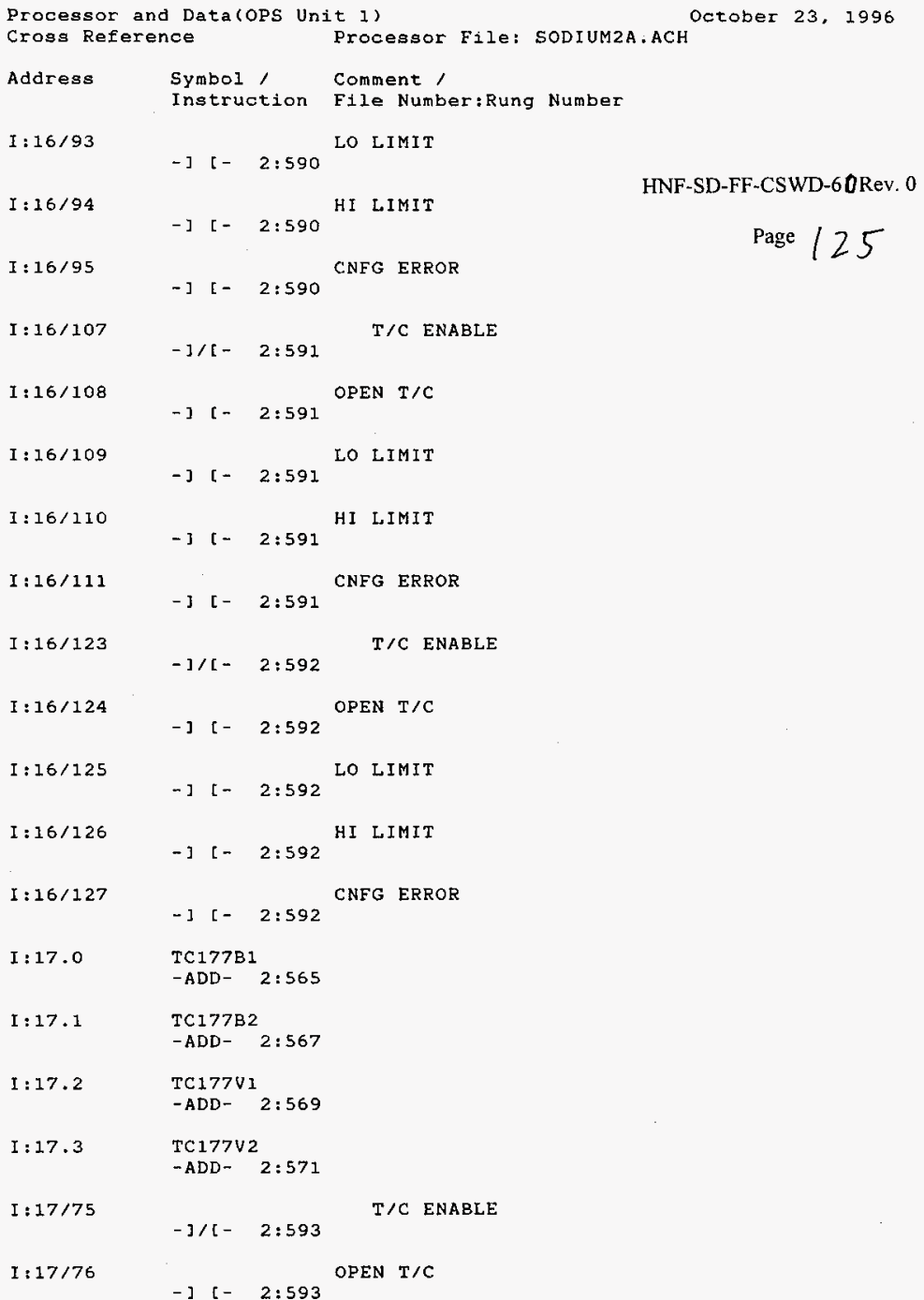$$
\text { CNFG ERROR }
$$

HNF-SD-FF-CSWD-60Rev. 0

\section{Page 125}




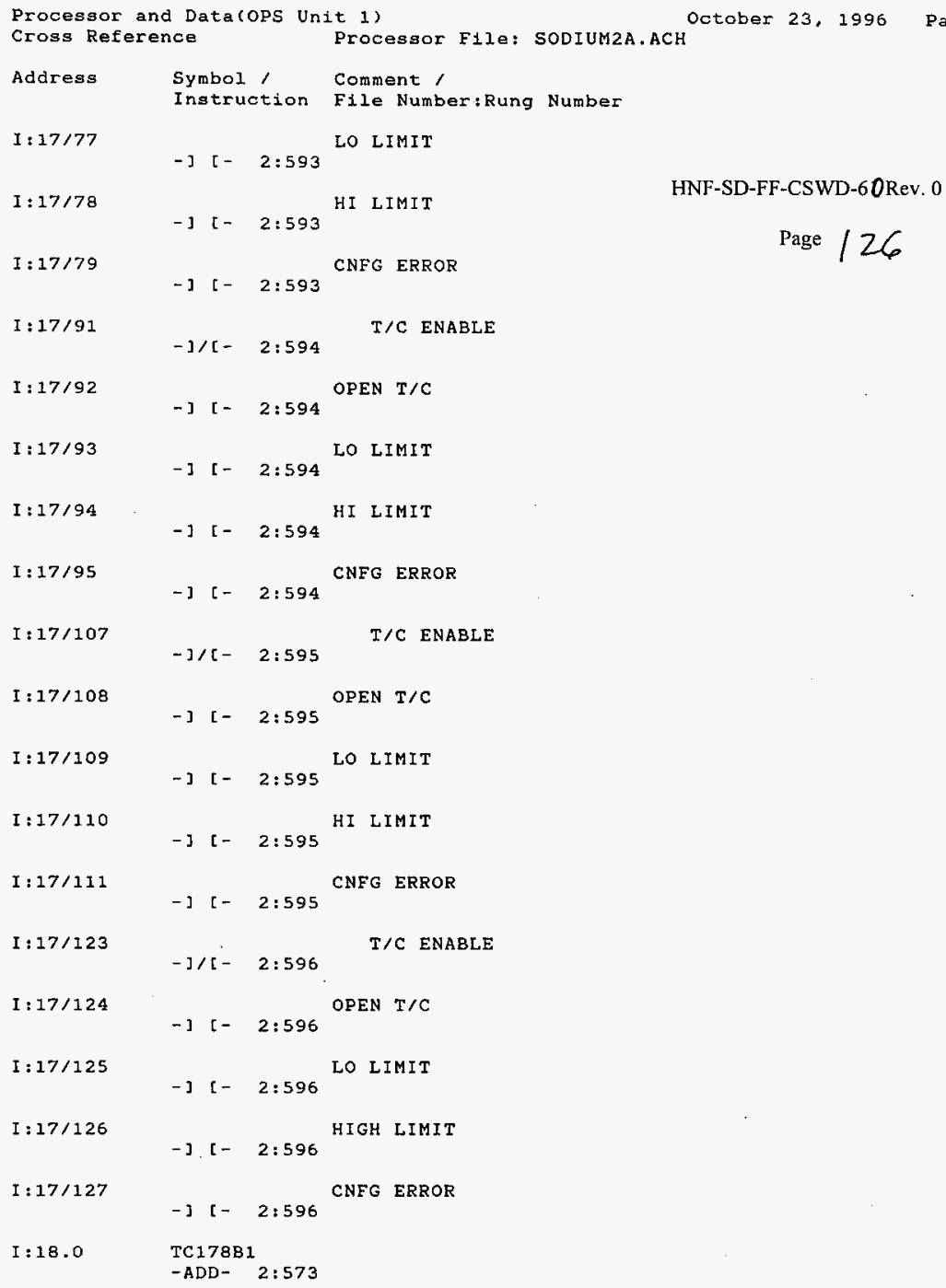

HNF-SD-FF-CSWD-60Rev. 0

Page / 26 


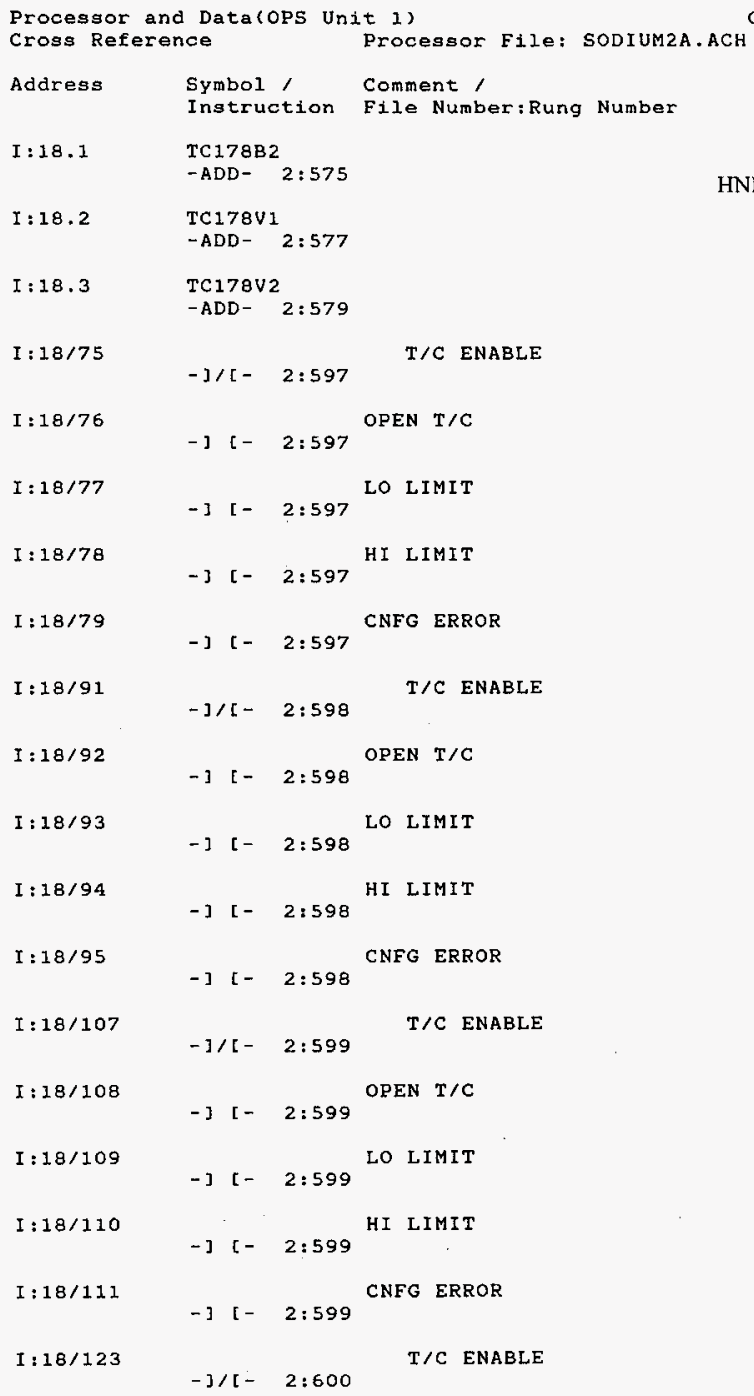

HNF-SD-FF-CSWD-60Rev. 0

\section{Page $/ 27$}




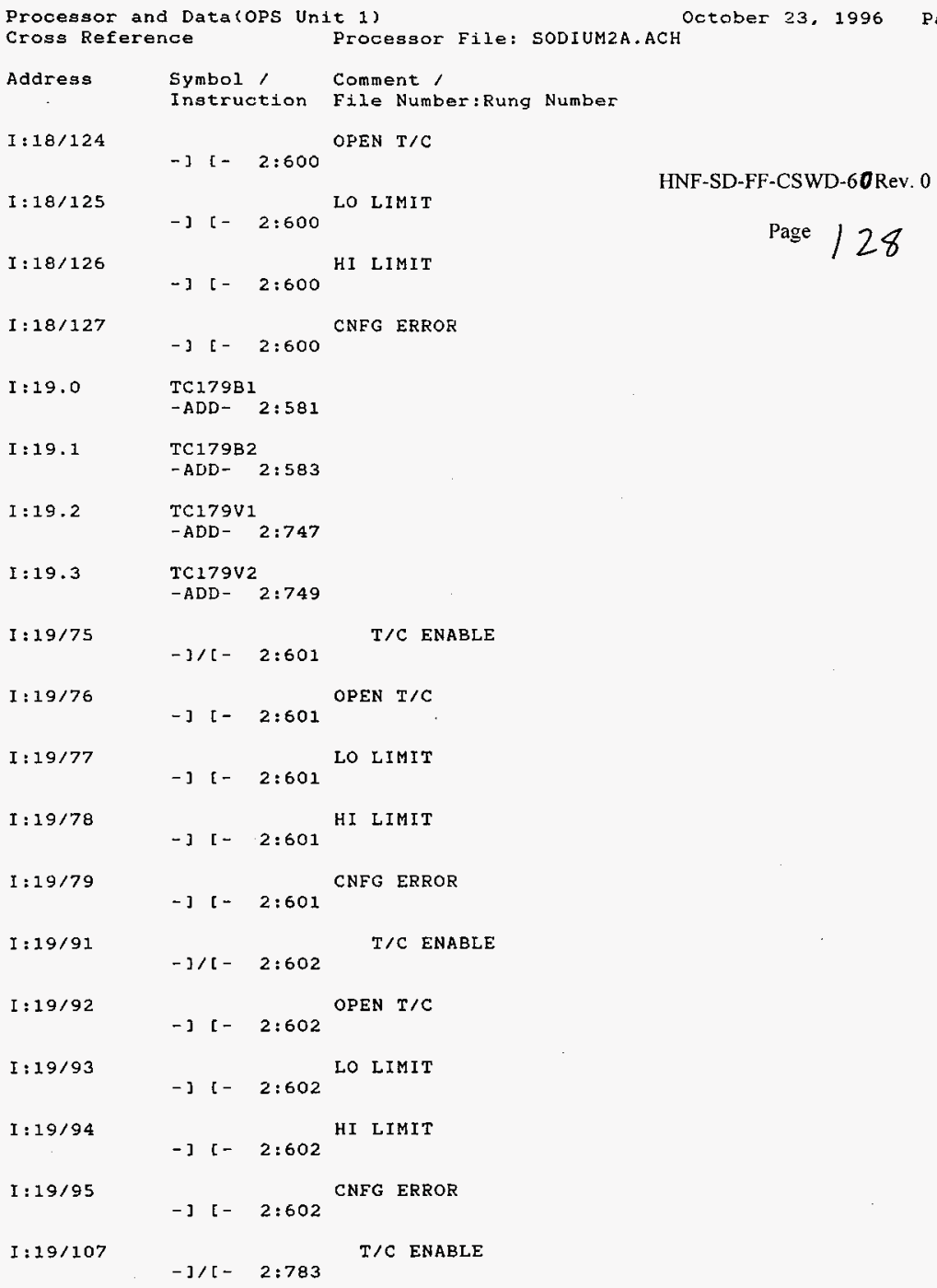

\section{HNF-SD-FF-CSWD-60 Rev. 0}

Page $/ 28$ 


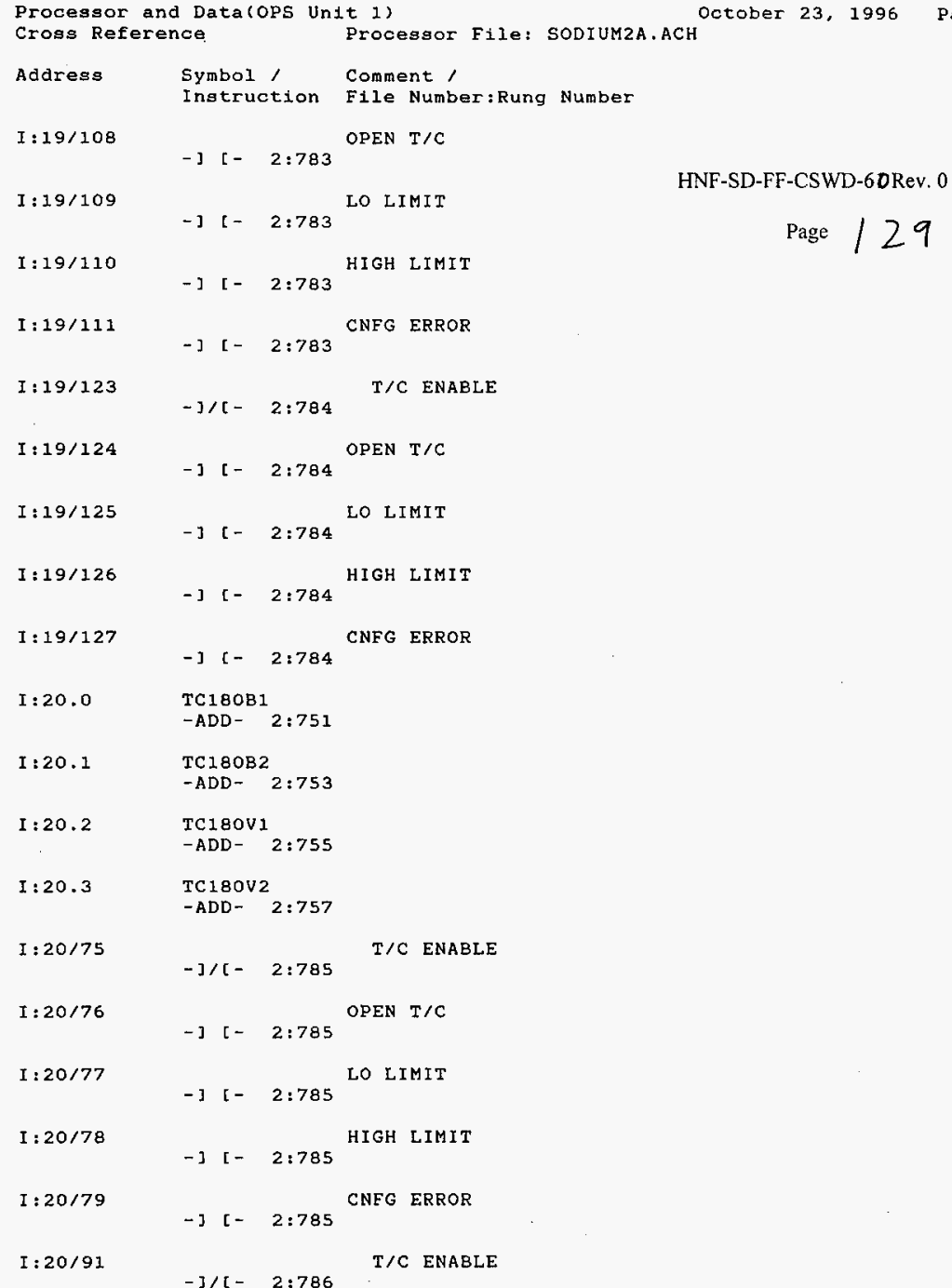

HNF-SD-FF-CSWD-60Rev. 0 


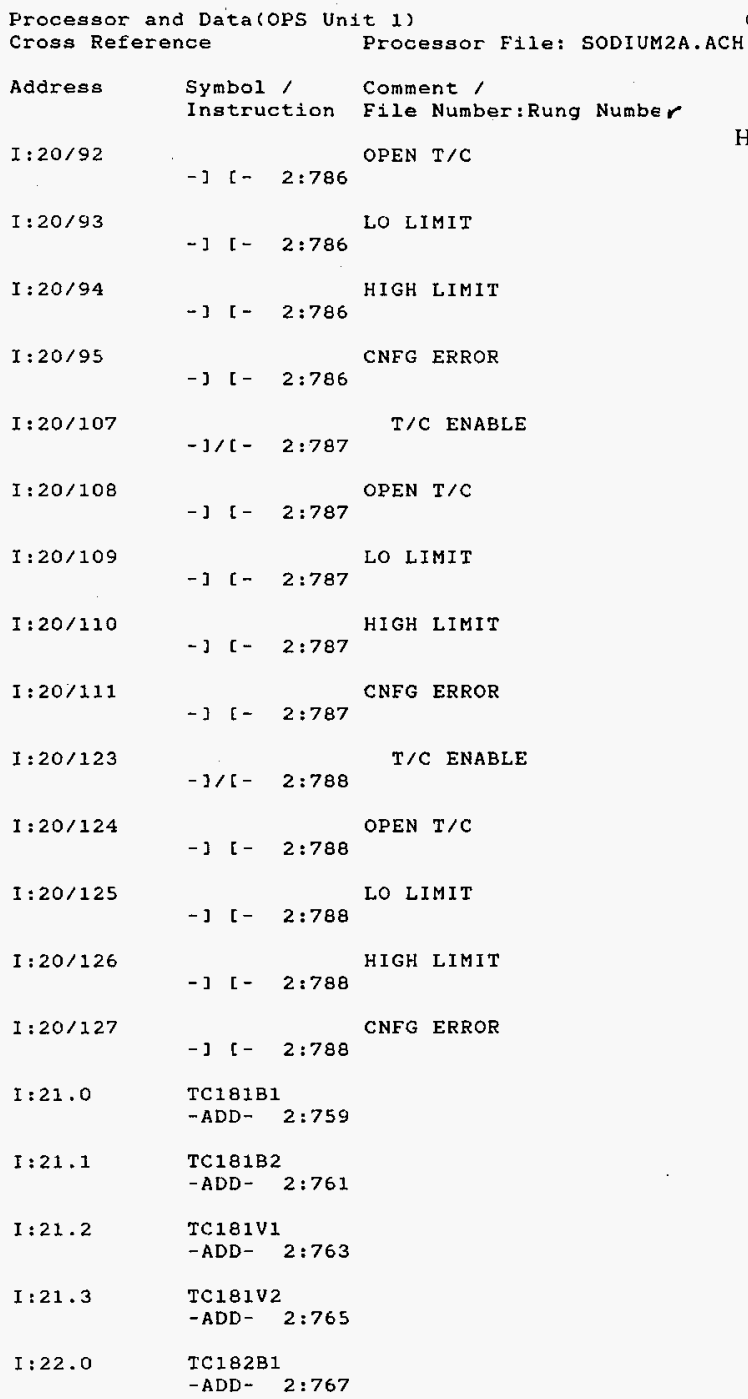




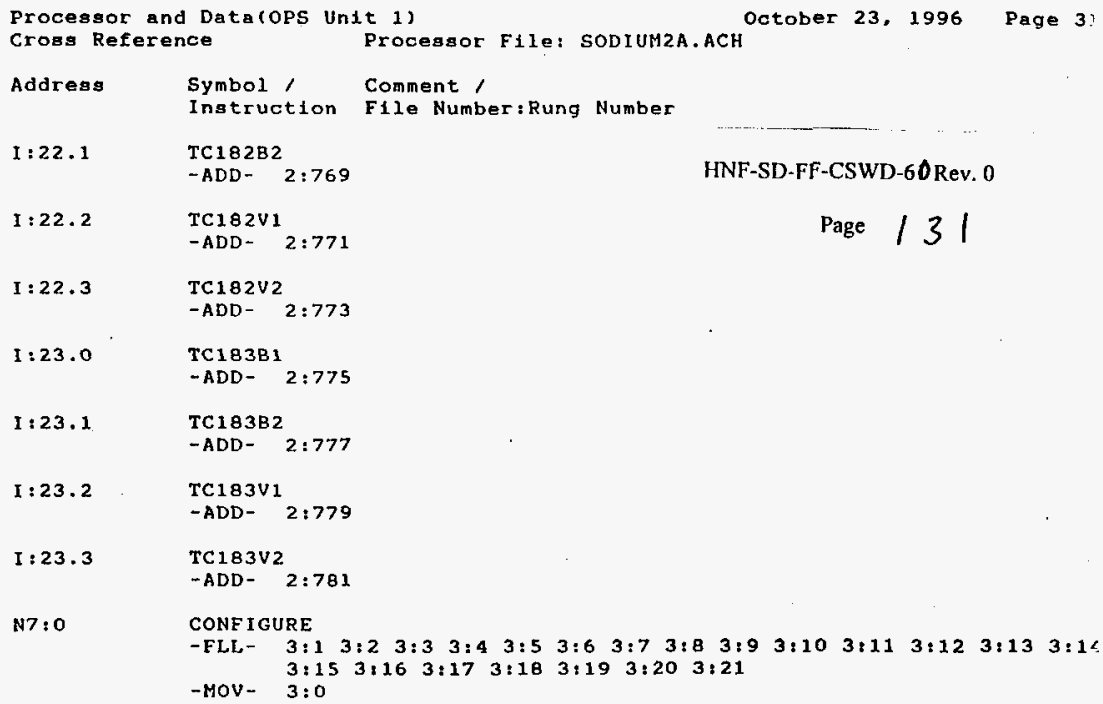

Address

Symbol, Comment /

Instruction File Number:Rung Number

$\mathrm{I}: 22.1$ TC182B2

$-A D D-\quad 2: 769$

HNF-SD-FF-CSWD-60 Rev. 0

$I: 22.2$

TC182V1

-ADD- 2:771

Page $\mid 31$

$I: 22.3 \quad T C 182 V 2$

-ADD- $2: 773$

I $: 23.0^{\circ}$ TC183BI

ADD- $2: 775$

$I: 23,1$

TC183B2

-ADD- 2:777

$I: 23.2$

TC183V1

-ADD- $2: 779$

$I: 23 \cdot 3$

TC183V2

-ADD- 2:781

N7:0

CONF IGURE

-FLL- $\quad 3: 1 \quad 3: 2 \quad 3: 3 \quad 3: 4 \quad 3: 5 \quad 3: 6 \quad 3: 7 \quad 3: 8 \quad 3: 9 \quad 3: 10 \quad 3: 11 \quad 3: 12 \quad 3: 13 \quad 3: 1<$ -MOV- $3: 0$ 
Tag Name Tag Type Description PLC Device PLC $\quad \underline{\text { LO Ladder Logic Rungs }}$

\begin{tabular}{|c|c|c|c|c|c|c|}
\hline \multirow[t]{4}{*}{ C143A } & $A A$ & ZONE 143A & DEV2:N7:1 & 3D0 & ADD & $2: 512: 170$ \\
\hline & & & & & GRT & $2: 522: 134$ \\
\hline & & & & & LES & $2: 134$ \\
\hline & & & & & MOV & $2: 52$ \\
\hline \multirow[t]{4}{*}{ C143B } & $A A$ & ZONE 143B & DEV2:N7:2 & 3D3 & ADD & $2: 532: 171$ \\
\hline & & & & & GRT & $2: 542: 135$ \\
\hline & & & & & LES & $2: 135$ \\
\hline & & & & & MOV & $2: 54$ \\
\hline \multirow[t]{4}{*}{ C144A } & AA & ZONE 144A & DEV2:N7:3 & 3D6 & ADD & $2: 552: 174$ \\
\hline & & & & & GRT & $2: 562: 137$ \\
\hline & & & & & LES & $2: 137$ \\
\hline & & & & & MOV & $2: 56$ \\
\hline \multirow[t]{4}{*}{ C144B } & $A A$ & ZONE 144B & DEV2:N7:4 & 3D9 & ADD & $2: 572: 175$ \\
\hline & & & & & GRT & $2: 582: 138$ \\
\hline & & & & & LES & $2: 138$ \\
\hline & & & & & MOV & $2: 58$ \\
\hline \multirow[t]{4}{*}{ C150A } & AA & ZONE 150A & DEV2:N7:5 & $3 E 0$ & ADD & $2: 592: 178$ \\
\hline & & & & & GRT & $2: 602: 140$ \\
\hline & & & & & LES & $2: 140$ \\
\hline & & & & & MOV & $2: 60$ \\
\hline \multirow[t]{4}{*}{ C150B } & $A A$ & ZONE 150B & DEV2:N7:6 & $3 \mathrm{E} 3$ & $\mathrm{ADD}$ & $2: 612: 179$ \\
\hline & & & & & GRT & $2: 622: 141$ \\
\hline & & & & & LES & $2: 141$ \\
\hline & & & & & MOV & $2: 62$ \\
\hline \multirow[t]{4}{*}{ C150C } & $A A$ & ZONE 150C & DEV2:N7:7 & $3 \mathrm{E} 6$ & ADD & $2: 632: 180$ \\
\hline & & & & & GRT & $2: 642: 142$ \\
\hline & & & & & LES & $2: 142$ \\
\hline & & & & & MOV & $2: 64$ \\
\hline \multirow[t]{4}{*}{ C151A } & $A A$ & ZONE 151A & DEV2:N7:8 & $3 E 9$ & ADD & $2: 652: 183$ \\
\hline & & & & & GRT & 2:66 2:144 \\
\hline & & & & & LES & $2: 144$ \\
\hline & & & & & MOV & $2: 66$ \\
\hline \multirow[t]{4}{*}{ C151B } & $A A$ & ZONE 151B & DEV2:N7:9 & $3 F 0$ & ADD & $2: 672: 184$ \\
\hline & & & & & GRT & $2: 682: 145$ \\
\hline & & & & & LES & $2: 145$ \\
\hline & & & & & MOV & $2: 68$ \\
\hline \multirow[t]{4}{*}{ C151C } & $A A$ & ZONE 151C & DEV2:N7:10 & $3 F 3$ & ADD & $2: 692: 185$ \\
\hline & & & & & GRT & $2: 702: 146$ \\
\hline & & & & & LES & $2: 148$ \\
\hline & & & & & MOV & $2: 70$ \\
\hline \multirow[t]{4}{*}{ C152A } & AA & ZONE 152A & DEV2:N7:11 & $3 \mathrm{~F} 6$ & ADD & $2: 712: 188$ \\
\hline & & & & & GRT & $2: 722: 148$ \\
\hline & & & & & LES & $2: 148$ \\
\hline & & & & & MOV & $2: 72$ \\
\hline \multirow[t]{4}{*}{ C152B } & AA & ZONE 152B & DEV2:N7:12 & $3 F 9$ & ADD & $2: 732: 189$ \\
\hline & & & & & GRT & $2: 742: 149$ \\
\hline & & & & & LES & $2: 149$ \\
\hline & & & & & MOV & $2: 74$ \\
\hline C153A & $A A$ & ZONE 153A & DEV2:N7:13 & $3 G 0$ & ADD & $2: 75$ 2:192 \\
\hline
\end{tabular}




\begin{tabular}{|c|c|c|c|c|c|c|}
\hline & & & & & GRT & $2: 762: 151$ \\
\hline & & & & & LES & $2: 151$ \\
\hline & & & & & MOV & $2: 76$ \\
\hline \multirow[t]{4}{*}{ C153B } & $A A$ & ZONE 153B & DEV2:N7:14 & $3 G 3$ & $A D D$ & $2: 772: 193$ \\
\hline & & & & & GRT & $2: 782: 152$ \\
\hline & & & & & LES & $2: 152$ \\
\hline & & & & & MOV & $2: 78$ \\
\hline \multirow[t]{4}{*}{ C154A } & AA & ZONE 154A & DEV2:N7:15 & $3 G 6$ & ADD & $2: 792: 196$ \\
\hline & & & & & GRT & $2: 802: 154$ \\
\hline & & & & & LES & $2: 154$ \\
\hline & & & & & MOV & $2: 80$ \\
\hline \multirow[t]{4}{*}{ C154B } & AA & ZONE 154B & DEV2:N7:16 & $3 G 9$ & ADD & $2: 812: 197$ \\
\hline & & & & & GRT & $2: 822: 155$ \\
\hline & & & & & LES & $2: 155$ \\
\hline & & & & & MOV & $2: 82$ \\
\hline \multirow[t]{4}{*}{ C160A } & AA & ZONE $160 A$ & DEV2:N7:17 & $3 \mathrm{HO}$ & $A D D$ & $2: 832: 200$ \\
\hline & & & & & GRT & $2: 842: 157$ \\
\hline & & & & & LES & $2: 157$ \\
\hline & & & & & MOV & $2: 84$ \\
\hline \multirow[t]{4}{*}{$\mathrm{C} 160 \mathrm{~B}$} & $\mathrm{AA}$ & ZONE $160 B$ & DEV2:N7:18 & $3 \mathrm{H} 3$ & ADD & $2: 852: 201$ \\
\hline & & & & & GRT & $2: 862: 158$. \\
\hline & & & & & LES & $2: 158$ \\
\hline & & & & & MOV & $2: 86$ \\
\hline \multirow[t]{4}{*}{ C160C } & $A A$ & ZONE $160 \mathrm{C}$ & DEV2:N7:19 & $3 \mathrm{H} 6$ & ADD & $2: 872: 202$ \\
\hline & & & & & GRT & $2: 882: 159$ \\
\hline & & & & & LES & $2: 159$ \\
\hline & & & & & MOV & $2: 88$ \\
\hline \multirow[t]{4}{*}{ C161A } & AA & ZONE $161 \mathrm{~A}$ & DEV2:N7:20 & $3 \mathrm{H} 9$ & $A D D$ & $2: 892: 205$ \\
\hline & & & & & GRT & $2: 902: 161$ \\
\hline & & & & & LES & $2: 161$ \\
\hline & & & & & MOV & $2: 90$ \\
\hline \multirow[t]{4}{*}{ C161B } & AA & ZONE 161B & DEV2:N7:21 & 310 & ADD & $2: 912: 206$ \\
\hline & & & & & GRT & $2: 922: 162$ \\
\hline & & & & & LES & $2: 162$ \\
\hline & & & & & MOV & $2: 92$ \\
\hline \multirow[t]{4}{*}{ C161C } & AA & ZONE 161C & DEV2:N7:22 & 313 & ADD & $2: 932: 207$ \\
\hline & & & & & GRT & $2: 942: 163$ \\
\hline & & & & & LES & $2: 163$ \\
\hline & & & & & MOV & $2: 94$ \\
\hline \multirow[t]{4}{*}{ C162A } & AA & ZONE $162 \mathrm{~A}$ & DEV2:N7:23 & 316 & ADD & $2: 952: 210$ \\
\hline & & & & & GRT & 2:96 2:165 \\
\hline & & & & & LES & $2: 165$ \\
\hline & & & & & MOV & $2: 96$ \\
\hline \multirow[t]{4}{*}{ C162B } & $A A$ & ZONE 162B & DEV2:N7:24 & 319 & ADD & $2: 972: 211$ \\
\hline & & & & & GRT & $2: 982: 166$ \\
\hline & & & & & LES & $2: 166$ \\
\hline & & & & & MOV & $2: 98$ \\
\hline \multirow[t]{2}{*}{ C163A } & AA & ZONE 163A & DEV2:N7:25 & 3J0 & $\mathrm{ADD}$ & $2: 992: 402$ \\
\hline & & & & & GRT & $2: 1002: 364$ \\
\hline
\end{tabular}




\begin{tabular}{|c|c|c|c|c|c|c|}
\hline & & & & & LES & $2: 364$ \\
\hline & & & & & MOV & $2: 100$ \\
\hline \multirow[t]{4}{*}{ C163B } & AA & ZONE 163B & DEV2:N7:26 & $3 \mathrm{~J} 3$ & $A D D$ & $2: 1012: 403$ \\
\hline & & & & & GRT & $2: 1022: 365$ \\
\hline & & & & & LES & $2: 365$ \\
\hline & & & & & MOV & $2: 102$ \\
\hline \multirow[t]{4}{*}{ C164A } & AA & ZONE 164A & DEV2:N7:27 & $3 J 6$ & ADD & $2: 1032: 406$ \\
\hline & & & & & GRT & $2: 1042: 367$ \\
\hline & & & & & LES & $2: 367$ \\
\hline & & & & & MOV & $2: 104$ \\
\hline \multirow[t]{4}{*}{ C164B } & AA & ZONE 164B & DEV2:N7:28 & $3 . J 9$ & ADD & $2: 1052: 407$ \\
\hline & & & & & GRT & $2: 1062: 368$ \\
\hline & & & & & LES & $2: 368$ \\
\hline & & & & & MOV & $2: 106$ \\
\hline \multirow[t]{4}{*}{ C17081 } & AA & ZONE 170B1 & DEV2:N7:29 & $3 \mathrm{~K} 0$ & ADD & $2: 2972: 410$ \\
\hline & & & & & GRT & $2: 2982: 370$ \\
\hline & & & & & LES & $2: 370$ \\
\hline & & & & & MOV & $2: 298$ \\
\hline \multirow[t]{4}{*}{$\mathrm{C} 170 \mathrm{~B} 2$} & AA & ZONE 170B2 & DEV2:N7:30 & $3 \mathrm{~K} 3$ & ADD & $2: 2992: 411$ \\
\hline & & & & & GRT & $2: 3002: 371$ \\
\hline & & & & & LES & $2: 371$ \\
\hline & & & & & MOV & $2: 300$ \\
\hline \multirow[t]{4}{*}{ C170V1 } & AA & ZONE 170V1 & DEV2:N7:31 & $3 K 6$ & ADD & $2: 3012: 414$ \\
\hline & & & & & GRT & $2: 3022: 373$ \\
\hline & & & & & LES & $2: 373$ \\
\hline & & & & & MOV & $2: 302$ \\
\hline \multirow[t]{4}{*}{ C170V2 } & AA & ZONE 170V2 & DEV2:N7:32 & $3 K 9$ & ADD & $2: 3032: 415$ \\
\hline & & & & & GRT & $2: 3042: 374$ \\
\hline & & & & & LES & $2: 374$ \\
\hline & & & & & MOV & $2: 304$ \\
\hline \multirow[t]{4}{*}{ C171B1 } & $A A$ & ZONE 171B1 & DEV2:N7:33 & 3LO & ADD & $2: 3052: 418$ \\
\hline & & & & & GRT & $2: 3062: 376$ \\
\hline & & & & & LES & $2: 376$ \\
\hline & & & & & MOV & $2: 306$ \\
\hline \multirow[t]{4}{*}{$\mathrm{C} 171 \mathrm{~B} 2$} & AA & ZONE 171B2 & DEV2:N7:34 & $3 \mathrm{~L} 3$ & ADD & $2: 3072: 419$ \\
\hline & & & & & GRT & $2: 3082: 377$ \\
\hline & & & & & LES & $2: 377$ \\
\hline & & & & & MOV & $2: 308$ \\
\hline \multirow[t]{4}{*}{ C171V1 } & AA & ZONE 171V1 & DEV2:N7:35 & $3 \mathrm{~L} 6$ & ADD & $2: 3092: 422$ \\
\hline & & & & & GRT & $2: 3102: 379$ \\
\hline & & & & & LES & $2: 379$ \\
\hline & & & & & MOV & $2: 310$ \\
\hline \multirow[t]{4}{*}{ C171V2 } & $A A$ & ZONE 171V2 & DEV2:N7:36 & $3 L 9$ & $\mathrm{ADD}$ & $2: 3112: 423$ \\
\hline & & & & & GRT & $2: 3122: 380$ \\
\hline & & & & & LES & $2: 380$ \\
\hline & & & & & MOV & $2: 312$ \\
\hline \multirow[t]{3}{*}{$\mathrm{C} 172 \mathrm{~B} 1$} & $A A$ & ZONE 172B1 & DEV2:N7:37 & $3 \mathrm{MO}$ & ADD & $2: 3132: 426$ \\
\hline & & & & & GRT & $2: 3142: 3762: 382$ \\
\hline & & & & & LES & $2: 3762: 382$ \\
\hline
\end{tabular}




\begin{tabular}{|c|c|c|c|c|c|c|}
\hline \multirow{5}{*}{$\mathrm{C} 172 \mathrm{~B} 2$} & & & & & MOV & $2: 314$ \\
\hline & $\mathrm{AA}$ & ZONE 172B2 & DEV2:N7:38 & $3 \mathrm{M} 3$ & ADD & $2: 3152: 427$ \\
\hline & & & & & GRT & $2: 3162: 383$ \\
\hline & & & & & LES & $2: 383$ \\
\hline & & & & & MOV & $2: 316$ \\
\hline \multirow[t]{4}{*}{ C172V1 } & $A A$ & ZONE $172 \mathrm{~V} 1$ & DEV2:N7:39 & $3 \mathrm{M} 6$ & ADD & $2: 3172: 430$ \\
\hline & & & & & GRT & $2: 3182: 385$ \\
\hline & & & & & LES & $2: 385$ \\
\hline & & & & & MOV & $2: 318$ \\
\hline \multirow[t]{4}{*}{$\mathrm{C}_{172 \mathrm{~V} 2}$} & $A A$ & ZONE $172 \mathrm{~V} 2$ & DEV2:N7:40 & $3 \mathrm{M} 9$ & ADD & $2: 3192: 431$ \\
\hline & & & & & GRT & $2: 3202: 386$ \\
\hline & & & & & LES & $2: 386$ \\
\hline & & & & & MOV & $2: 320$ \\
\hline \multirow[t]{4}{*}{ C173B1 } & $A A$ & ZONE 173B1 & DEV2:N7:41 & $4 A O$ & ADD & $2: 3212: 434$ \\
\hline & & & & & GRT & $2: 3222: 388$ \\
\hline & & & & & LES & $2: 388$ \\
\hline & & & & & MOV & $2: 322$ \\
\hline \multirow[t]{4}{*}{$\mathrm{C} 173 \mathrm{~B} 2$} & $A A$ & ZONE $173 B 2$ & DEV2:N7:42 & $4 A 3$ & ADD & $2: 3232: 435$ \\
\hline & & & & & GRT & $2: 3242: 389$ \\
\hline & & & & & LES & $2: 389$ \\
\hline & & & & & MOV & $2: 324$ \\
\hline \multirow[t]{4}{*}{ C173V1 } & $A A$ & ZONE 173V1 & DEV2:N7:43 & $4 A 6$ & ADD & $2: 3252: 438$ \\
\hline & & & & & GRT & $2: 3262: 391$ \\
\hline & & & & & LES & $2: 391$ \\
\hline & & & & & MOV & $2: 326$ \\
\hline \multirow[t]{4}{*}{ C173V2 } & $A A$ & ZONE 173V2 & DEV2:N7:44 & $4 A 9$ & ADD & $2: 3272: 439$ \\
\hline & & & & & GRT & $2: 3282: 392$ \\
\hline & & & & & LES & $2: 392$ \\
\hline & & & & & MOV & $2: 328$ \\
\hline \multirow[t]{4}{*}{$\mathrm{C} 174 \mathrm{~B} 1$} & $A A$ & ZONE 174B1 & DEV2:N7:45 & $4 \mathrm{BO}$ & $A D D$ & $2: 329$ 2:442 \\
\hline & & & & & GRT & $2: 3302: 394$ \\
\hline & & & & & LES & $2: 394$ \\
\hline & & & & & MOV & $2: 330$ \\
\hline \multirow[t]{4}{*}{ C174B2 } & $A A$ & ZONE 174B2 & DEV2:N7:46 & 483 & $A D D$ & $2: 3312: 443$ \\
\hline & & & & & GRT & $2: 3322: 395$ \\
\hline & & & & & LES & $2: 395$ \\
\hline & & & & & MOV & $2: 332$ \\
\hline \multirow[t]{4}{*}{ C174V1 } & $A A$ & ZONE 174V1 & DEV2:N7:47 & $4 \mathrm{~B} 6$ & ADD & $2: 3332: 446$ \\
\hline & & & & & GRT & $2: 3342: 397$ \\
\hline & & & & & LES & $2: 397$ \\
\hline & & & & & MOV & $2: 334$ \\
\hline \multirow[t]{4}{*}{ C174V2 } & $A A$ & ZONE $174 \mathrm{~V} 2$ & DEV2:N7:48 & $4 \mathrm{~B} 9$ & $A D D$ & $2: 3352: 447$ \\
\hline & & & & & GRT & $2: 3362: 398$ \\
\hline & & & & & LES & $2: 398$ \\
\hline & & & & & MOV & $2: 336$ \\
\hline \multirow[t]{4}{*}{ C175B1 } & $A A$ & ZONE 175B1 & DEV2:N7:49 & $4 \mathrm{CO}$ & ADD & $2: 5492: 635$ \\
\hline & & & & & GRT & $2: 5502: 606$ \\
\hline & & & & & LES & $2: 606$ \\
\hline & & & & & MOV & $2: 550$ \\
\hline
\end{tabular}

HNF-SD-FF-CSWD-60 Rev. 0 Page $/ 35$ 
Tag Name Tag Type Description $\underline{\text { PLC Device PLC I/O Ladder Logic Rungs }}$

\begin{tabular}{|c|c|c|c|c|c|c|}
\hline C17582 & $A A$ & ZONE $175 B 2$ & DEV2:N7:50 & $4 \mathrm{C} 3$ & ADD & $2: 5512: 636$ \\
\hline & & & & & GRT & $2: 5522: 607$ \\
\hline & & & & & LES & $2: 607$ \\
\hline & & & & & MOV & $2: 552$ \\
\hline C175V1 & $A A$ & ZONE $175 \mathrm{~V} 1$ & DEV2:N7:51 & $4 \mathrm{C} 6$ & ADD & $2: 5532: 639$ \\
\hline & & & & & GRT & $2: 5542: 609$ \\
\hline & & & & & LES & $2: 609$ \\
\hline & & & & & MOV & $2: 554$ \\
\hline C175V2 & $A A$ & ZONE $175 \mathrm{~V} 2$ & DEV2:N7:52 & $4 \mathrm{C9}$ & ADD & $2: 5552: 640$ \\
\hline & & & & & GRT & $2: 5562: 610$ \\
\hline & & & & & LES & $2: 610$ \\
\hline & & & & & MOV & $2: 556$ \\
\hline $\mathrm{C} 176 \mathrm{~B} 1$ & $A A$ & ZONE 176B1 & DEV2:N7:53 & $4 \mathrm{DO}$ & ADD & $2: 5572: 643$ \\
\hline & & & & & GRT & $2: 5582: 612$ \\
\hline & & & & & LES & $2: 612$ \\
\hline & & & & & MOV & $2: 558$ \\
\hline C17682 & AA & ZONE 17682 & DEV2:N7:54 & 4D3 & $A D D$ & $2: 5592: 644$ \\
\hline & & & & & GRT & $2: 5602: 613$ \\
\hline & & & & & LES & $2: 613$ \\
\hline & & & & & MOV & $2: 560$ \\
\hline C176V1 & $A A$ & ZONE $176 \mathrm{~V} 1$ & DEV2:N7:55 & 4D6 & ADD & 2:561 2:647 \\
\hline & & & & & GRT & $2: 5622: 615$ \\
\hline & & & & & LES & $2: 615$ \\
\hline & & & & & MOV & $2: 562$ \\
\hline C176V2 & AA & ZONE $176 V 2$ & DEV2:N7:56 & 4D9 & ADD & $2: 5632: 648$ \\
\hline & & & & & GRT & $2: 5642: 616$ \\
\hline & & & & & LES & $2: 616$ \\
\hline & & & & & MOV & $2: 564$ \\
\hline C177B1 & $\mathrm{AA}$ & ZONE 177B1 & DEV2:N7:57 & 4E0 & ADD & $2: 5652: 651$ \\
\hline & & & & & GRT & $2: 5662: 618$ \\
\hline & & & & & LES & $2: 618$ \\
\hline & & & & & MOV & $2: 566$ \\
\hline $\mathrm{C} 177 \mathrm{~B} 2$ & $\mathrm{AA}$ & ZONE 177B2 & DEV2:N7:58 & 4E3 & ADD & $2: 5672: 652$ \\
\hline & & & & & GRT & $2: 5682: 619$ \\
\hline & & & & & LES & $2: 619$ \\
\hline & & & & & MOV & $2: 568$ \\
\hline C177V1 & AA & ZONE 177V1 & DEV2:N7:59 & $4 \mathrm{E} 6$ & ADD & $2: 5692: 655$ \\
\hline & & & & & GRT & $2: 5702: 621$ \\
\hline & & & & & LES & $2: 621$ \\
\hline & & & & & MOV & $2: 570$ \\
\hline C177V2 & AA & ZONE $177 \mathrm{~V} 2$ & DEV2:N7:60 & 4E9 & $\mathrm{ADD}$ & $2: 5712: 656$ \\
\hline & & & & & GRT & $2: 5722: 622$ \\
\hline & & & & & LES & $2: 622$ \\
\hline & & & & & MOV & $2: 572$ \\
\hline C178B1 & AA & ZONE 178B1 & DEV2:N7:61 & 4F0 & ADD & $2: 5732: 659$ \\
\hline & & & & & GRT & $2: 5742: 624$ \\
\hline & & & & & LES & $2: 624$ \\
\hline & & & & & MOV & $2: 574$ \\
\hline & AA & ZONE $178 \mathrm{~B}$ & DEV2:N7:62 & $4 \mathrm{~F} 3$ & ADD & $2: 5752: 660$ \\
\hline
\end{tabular}

HNF-SD-FF-CSWD-60 Rev. o Page 136 


\begin{tabular}{|c|c|c|c|c|c|c|}
\hline & & & & & GRT & $2: 5762: 625$ \\
\hline & & & & & LES & $2: 625$ \\
\hline & & & & & MOV & $2: 576$ \\
\hline \multirow[t]{4}{*}{ C178V1 } & AA & ZONE $178 \mathrm{~V} 1$ & DEV2:N7:63 & $4 \mathrm{F6}$ & ADD & $2: 5772: 663$ \\
\hline & & & & & GRT & $2: 5782: 627$ \\
\hline & & & & & LES & $2: 627$ \\
\hline & & & & & MOV & $2: 578$ \\
\hline \multirow[t]{4}{*}{ C178V2 } & $A A$ & ZONE 178V2 & DEV2:N7:64 & $4 \mathrm{Fg}$ & ADD & $2: 5792: 664$ \\
\hline & & & & & GRT & $2: 5802: 628$ \\
\hline & & & & & LES & $2: 628$ \\
\hline & & & & & MOV & $2: 580$ \\
\hline \multirow[t]{4}{*}{ C179B1 } & $A A$ & ZONE 179B1 & DEV2:N7:65 & $4 G 0$ & ADD & $2: 5812: 667$ \\
\hline & & & & & GRT & $2: 5822: 630$ \\
\hline & & & & & LES & $2: 630$ \\
\hline & & & & & MOV & $2: 582$ \\
\hline \multirow[t]{4}{*}{ C179B2 } & $A A$ & ZONE $179 \mathrm{~B} 2$ & DEV2:N7:66 & $4 \mathrm{G} 3$ & ADD & $2: 5832: 668$ \\
\hline & & & & & GRT & $2: 5842: 631$ \\
\hline & & & & & LES & $2: 631$ \\
\hline & & & & & MOV & $2: 584$ \\
\hline \multirow[t]{4}{*}{ C179V1 } & $A A$ & ZONE 179V1 & DEV2:N7:67 & $4 \mathrm{G} 6$ & ADD & $2: 7472: 833$ \\
\hline & & & & & GRT & $2: 7482: 804$ \\
\hline & & & & & LES & $2: 804$ \\
\hline & & & & & MOV & $2: 748$ \\
\hline \multirow[t]{4}{*}{ C179V2 } & $A A$ & ZONE 179V2 & DEV2:N7:68 & $4 \mathrm{G} 9$ & ADD & $2: 7492: 834$ \\
\hline & & & & & GRT & $2: 7502: 805$ \\
\hline & & & & & LES & $2: 805$ \\
\hline & & & & & MOV & $2: 750$ \\
\hline \multirow[t]{4}{*}{ C180B1 } & $A A$ & ZONE 180B1 & DEV2:N7:69 & $4 \mathrm{HO}$ & ADD & $2: 7512: 837$ \\
\hline & & & & & GRT & $2: 7522: 807$ \\
\hline & & & & & LES & $2: 807$ \\
\hline & & & & & MOV & $2: 752$ \\
\hline \multirow[t]{4}{*}{ C180B2 } & $A A$ & ZONE 180B2 & DEV2:N7:70 & $4 \mathrm{H} 3$ & ADD & $2: 7532: 838$ \\
\hline & & & & & GRT & $2: 7542: 808$ \\
\hline & & & & & LES & $2: 808$ \\
\hline & & & & & MOV & $2: 754$ \\
\hline \multirow[t]{4}{*}{ C180V1 } & $A A$ & ZONE 180V1 & DEV2:N7:71 & $4 \mathrm{H} 6$ & ADD & $2: 7552: 841$ \\
\hline & & & & & GRT & $2: 7562: 810$ \\
\hline & & & & & LES & $2: 810$ \\
\hline & & & & & MOV & $2: 756$ \\
\hline \multirow[t]{4}{*}{ C180V2 } & $A A$ & ZONE $180 \mathrm{~V} 2$ & DEV2:N7:72 & $4 \mathrm{H} 9$ & ADD & $2: 7572: 842$ \\
\hline & & & & & GRT & $2: 7582: 811$ \\
\hline & & & & & LES & $2: 811$ \\
\hline & & & & & MOV & $2: 758$ \\
\hline \multirow[t]{4}{*}{ C181B1 } & $A A$ & ZONE 181B1 & DEV2:N7:73 & 410 & ADD & $2: 7592: 845$ \\
\hline & & & & & GRT & $2: 7602: 813$ \\
\hline & & & & & LES & $2: 813$ \\
\hline & & & & & MOV & $2: 760$ \\
\hline \multirow[t]{2}{*}{ C181B2 } & $A A$ & ZONE 181B2 & DEV2:N7:74 & 413 & $A D D$ & $2: 7612: 846$ \\
\hline & & & & & GRT & $2: 7622: 814$ \\
\hline
\end{tabular}




\begin{tabular}{|c|c|c|c|c|c|c|}
\hline & & & & & LES & $2: 814$ \\
\hline & & & & & MOV & $2: 762$ \\
\hline \multirow[t]{4}{*}{ C181V1 } & AA & ZONE 181V1 & DEV2:N7:75 & 416 & ADD & 2:763 2:849 \\
\hline & & & & & GRT & $2: 7642: 816$ \\
\hline & & & & & LES & $2: 816$ \\
\hline & & & & & MOV & $2: 764$ \\
\hline \multirow[t]{4}{*}{ C181V2 } & $A A$ & ZONE 181V2 & DEV2:N7:76 & 419 & $\mathrm{ADD}$ & $2: 7652: 850$ \\
\hline & & & & & GRT & $2: 7662: 817$ \\
\hline & & & & & LES & $2: 817$ \\
\hline & & & & & MOV & $2: 766$ \\
\hline \multirow[t]{4}{*}{ C182B1 } & $A A$ & ZONE 182B1 & DEV2:N7:77 & 4JO & $A D D$ & $2: 7672: 853$ \\
\hline & & & & & GRT & $2: 7682: 819$ \\
\hline & & & & & LES & $2: 819$ \\
\hline & & & & & MOV & $2: 768$ \\
\hline \multirow[t]{4}{*}{ C182B2 } & $A A$ & ZONE $182 B 2$ & DEV2:N7:78 & $4 \mathrm{~J} 3$ & ADD & $2: 7692: 854$ \\
\hline & & & & & GRT & $2: 7702: 820$ \\
\hline & & & & & LES & $2: 820$ \\
\hline & & & & & MOV & $2: 770$ \\
\hline \multirow[t]{4}{*}{ C182V1 } & $A A$ & ZONE $182 \mathrm{~V} 1$ & DEV2:N7:79 & $4 J 6$ & ADD & 2:771 2:857 \\
\hline & & & & & GRT & $2: 7722: 822$ \\
\hline & & & & & LES & $2: 822$ \\
\hline & & & & & MOV & $2: 772$ \\
\hline \multirow[t]{4}{*}{$\mathrm{C} 182 \mathrm{~V} 2$} & $\mathrm{AA}$ & ZONE $182 \mathrm{~V} 2$ & DEV2:N7:81 & $4 \mathrm{~J} 9$ & ADD & $2: 7732: 858$ \\
\hline & & & & & GRT & $2: 7742: 823$ \\
\hline & & & & & LES & $2: 823$ \\
\hline & & & & & MOV & $2: 774$ \\
\hline \multirow[t]{4}{*}{ C183B1 } & AA & ZONE 183B1 & DEV2:N7:80 & $4 \mathrm{KO}$ & $A D D$ & $2: 7752: 861$ \\
\hline & & & & & GRT & $2: 7762: 825$ \\
\hline & & & & & LES & $2: 825$ \\
\hline & & & & & MOV & $2: 776$ \\
\hline \multirow[t]{4}{*}{$\mathrm{C} 183 \mathrm{~B} 2$} & $A A$ & ZONE $183 B 2$ & DEV2:N7:82 & $4 K 3$ & ADD & $2: 7772: 862$ \\
\hline & & & & & GRT & $2: 7782: 826$ \\
\hline & & & & & LES & $2: 826$ \\
\hline & & & & & MOV & $2: 778$ \\
\hline \multirow[t]{4}{*}{ C183V1 } & AA & ZONE 183V1 & DEV2:N7:83 & $4 \mathrm{~K} 6$ & ADD & $2: 7792: 865$ \\
\hline & & & & & GRT & $2: 7802: 828$ \\
\hline & & & & & LES & $2: 828$ \\
\hline & & & & & MOV & $2: 780$ \\
\hline \multirow[t]{4}{*}{ C183V2 } & $A A$ & ZONE $183 \mathrm{~V} 2$ & DEV2:N7:84 & $4 K 9$ & ADD & $2: 7812: 866$ \\
\hline & & & & & GRT & $2: 7822: 829$ \\
\hline & & & & & LES & $2: 829$ \\
\hline & & & & & MOV & $2: 782$ \\
\hline
\end{tabular}

COM2 AA COM FAIL PLC 2 DEV2:N10:89 
Processor and Data(OPS Unit 1$)$

Cross Reference

Processor File: SODIUM2A.ACH

Address Symbol / Comment /

Instruction File Number:Rung Number

N7 : $88 \quad$ COUNT

\begin{tabular}{|c|c|c|c|c|c|c|c|c|c|c|}
\hline ADD- & $2: 170$ & $: 170$ & 171 & 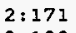 & 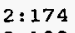 & & & 0 & & \\
\hline & $2: 179$ & $: 179$ & $: 180$ & $: 180$ & $2: 183$ & $2: 183$ & $2: 184$ & $2: 184$ & $2: 185$ & $2: 185$ \\
\hline & $2: 188$ & $: 188$ & $: 189$ & $: 189$ & $2: 192$ & $2: 192$ & $: 193$ & $2: 193$ & $2: 196$ & $2: 196$ \\
\hline & $2: 197$ & $: 197$ & 200 & $2: 200$ & $: 201$ & 201 & 202 & $: 202$ & $2: 205$ & $2: 205$ \\
\hline & $2: 206$ & $: 206$ & $: 207$ & $2: 207$ & $: 210$ & 210 & 211 & $: 211$ & $2: 402$ & $2: 402$ \\
\hline & $2: 403$ & $: 403$ & $: 406$ & $2: 406$ & $: 407$ & $: 407$ & 410 & 1410 & $2: 411$ & $2: 411$ \\
\hline & $2: 414$ & $2: 414$ & $2: 415$ & $2: 415$ & $: 418$ & $: 418$ & : 419 & $: 419$ & $2: 422$ & $2: 422$ \\
\hline & $2: 423$ & $2: 423$ & $2: 426$ & $2: 426$ & $: 427$ & $2: 427$ & $: 430$ & 430 & $2: 431$ & $2: 431$ \\
\hline & $2: 434$ & $2: 434$ & $2: 435$ & $2: 435$ & $2: 438$ & $2: 438$ & $: 439$ & $2: 439$ & $2: 442$ & $2: 442$ \\
\hline & $2: 443$ & $2: 443$ & $2: 446$ & $2: 446$ & $2: 447$ & $2: 447$ & $: 635$ & $: 635$ & $2: 636$ & $2: 636$ \\
\hline & $2: 639$ & $2: 639$ & $2: 640$ & $2: 640$ & $: 643$ & $: 643$ & 644 & 644 & $2: 647$ & $2: 647$ \\
\hline & $2: 648$ & $2: 648$ & $2: 651$ & $2: 651$ & $2: 652$ & $2: 652$ & 655 & $2: 655$ & $2: 656$ & $2: 656$ \\
\hline & $2: 659$ & $2: 659$ & $2: 660$ & $2: 660$ & 663 & 663 & 664 & 664 & $2: 667$ & $2: 667$ \\
\hline & $2: 668$ & $2: 668$ & $2: 833$ & $2: 833$ & $2: 834$ & $2: 834$ & 837 & 337 & $2: 838$ & $2: 838$ \\
\hline & $2: 841$ & $: 841$ & $2: 842$ & $2: 842$ & 345 & $2: 845$ & 946 & 46 & 49 & $2: 849$ \\
\hline & $2: 850$ & $2: 850$ & $2: 853$ & $2: 853$ & 854 & 4 & 7 & 57 & 58 & $2: 858$ \\
\hline & $2: 861$ & $: 861$ & $2: 862$ & $2: 862$ & 865 & & & $2: 866$ & & \\
\hline 4 & $2: 169$ & $2: 173$ & $2: 177$ & $2: 182$ & $2: 187$ & 1 & 5 & $2: 199$ & $2: 204$ & $2: 209$ \\
\hline & $2: 401$ & $2: 405$ & $2: 409$ & $2: 413$ & $2: 417$ & 2: & 5 & 429 & $2: 433$ & $2: 437$ \\
\hline & $2: 441$ & $2: 445$ & $2: 634$ & $2: 638$ & $2: 642$ & $2: 6$ & 0 & 654 & $2: 658$ & $2: 662$ \\
\hline & $2: 666$ & $2: 832$ & $2: 836$ & $2: 840$ & $2: 844$ & $2: 848$ & $2: 8$ & $: 856$ & $2: 860$ & $2: 864$ \\
\hline 18 & $2: 172$ & $2: 176$ & $2: 181$ & $2: 186$ & $2: 190$ & $2: 194$ & 2: & $2: 203$ & $2: 208$ & $2: 212$ \\
\hline & $2: 404$ & $2: 408$ & $2: 412$ & $2: 416$ & $2: 420$ & $2: 424$ & $2: 428$ & $2: 432$ & $2: 436$ & $2: 440$ \\
\hline & $2: 444$ & $2: 448$ & $2: 637$ & $2: 641$ & $2: 645$ & $2: 649$ & $2: 6$ & $2: 657$ & $2: 661$ & $2: 665$ \\
\hline & $2: 669$ & $2: 835$ & : 839 & $2: 843$ & $2: 847$ & $2: 851$ & 5 & $2: 859$ & $2: 863$ & $2: 867$ \\
\hline
\end{tabular}

HNF-SD-FF-CSWD-60 Rev. 0

Page / 39 


\begin{tabular}{|c|c|c|c|c|c|c|}
\hline AV143 & $\mathrm{Al}$ & ZONE 143 Average & DEV2:N7:91 & 4LOO & ADD & $2: 133$ \\
\hline & & & & & DIV & $2: 172$ \\
\hline & & & & & MOV & $2: 42: 42: 215$ \\
\hline & & & & & PID & $2: 220$ \\
\hline & & & & & SUB & $2: 133$ \\
\hline AV144 & Al & ZONE 144 Average & DEV2:N7:92 & 4L.01 & $\mathrm{ADD}$ & $2: 136$ \\
\hline & & & & & DIV & $2: 176$ \\
\hline & & & & & MOV & $2: 52: 52: 223$ \\
\hline & & & & & PID & $2: 228$ \\
\hline & & & & & SUB & $2: 136$ \\
\hline AV150 & $\mathrm{Al}$ & ZONE 150 Average & DEV2:N7:93 & 4LO2 & ADD & $2: 139$ \\
\hline & & & & & DIV & $2: 181$ \\
\hline & & & & & MOV & $2: 62: 62: 231$ \\
\hline & & & & & PID & $2: 236$ \\
\hline & & & & & SUB & $2: 139$ \\
\hline AV151 & Al & ZONE 151 Average & DEV2:N7:94 & 4L03 & ADD & $2: 143$ \\
\hline & & & & & DIV & $2: 186$ \\
\hline & & & & & MOV & $2: 72: 72: 239$ \\
\hline & & & & & PID & $2: 244$ \\
\hline & & & & & SUB & $2: 143$ \\
\hline AV152 & Al & ZONE 152 Average & DEV2:N7:95 & 4L04 & $\mathrm{ADD}$ & $2: 147$ \\
\hline & & & & & DIV & $2: 190$ \\
\hline & & & & & MOV & $2: 8 \quad 2: 8 \quad 2: 247$ \\
\hline & & & & & PID & $2: 252$ \\
\hline & & & & & SUB & $2: 147$ \\
\hline AV153 & $\mathrm{Al}$ & ZONE 153 Average & DEV2:N7:96 & 4L05 & ADD & $2: 150$ \\
\hline & & & & & DIV & $2: 194$ \\
\hline & & & & & MOV & $2: 92: 92: 255$ \\
\hline & & & & & PID & $2: 260$ \\
\hline & & & & & SUB & $2: 150$ \\
\hline AV154 & $\mathrm{Al}$ & ZONE 154 Average & DEV2:N7:97 & 4L06 & ADD & $2: 153$ \\
\hline & & & & & DIV & $2: 198$ \\
\hline & & & & & MOV & $2: 102: 102: 263$ \\
\hline & & & & & PID & $2: 268$ \\
\hline & & & & & SUB & $2: 153$ \\
\hline AV160 & $\mathrm{Al}$ & ZONE 160 Average & DEV2:N7:98 & 4L07 & ADD & $2: 156$ \\
\hline & & & & & DIV & $2: 203$ \\
\hline & & & & & MOV & 2:11 2:11 2:271 \\
\hline & & & & & PID & $2: 276$ \\
\hline & & & & & SUB & $2: 156$ \\
\hline AV161 & $\mathrm{Al}$ & ZONE 161 Average & DEV2:N7:99 & 4L08 & ADD & $2: 160$ \\
\hline & & & & & DIV & $2: 208$ \\
\hline & & & & & MOV & $2: 122: 122: 279$ \\
\hline & & & & & PID & $2: 284$ \\
\hline & & & & & SUB & $2: 160$ \\
\hline AV162 & $\mathrm{Al}$ & ZONE 162 Average & DEV2:N7:100 & 4L.09 & $A D D$ & $2: 164$ \\
\hline & & & & & DIV & 2:212 \\
\hline & & & & & MOV & $2: 132: 132: 288$ \\
\hline & & & & & PID & $2: 293$ \\
\hline & & & & & SUB & $2: 164$ \\
\hline AV163 & Al & ZONE 163 Average & DEV2:N7:101 & $4 L 10$ & ADD & $2: 363$ \\
\hline
\end{tabular}




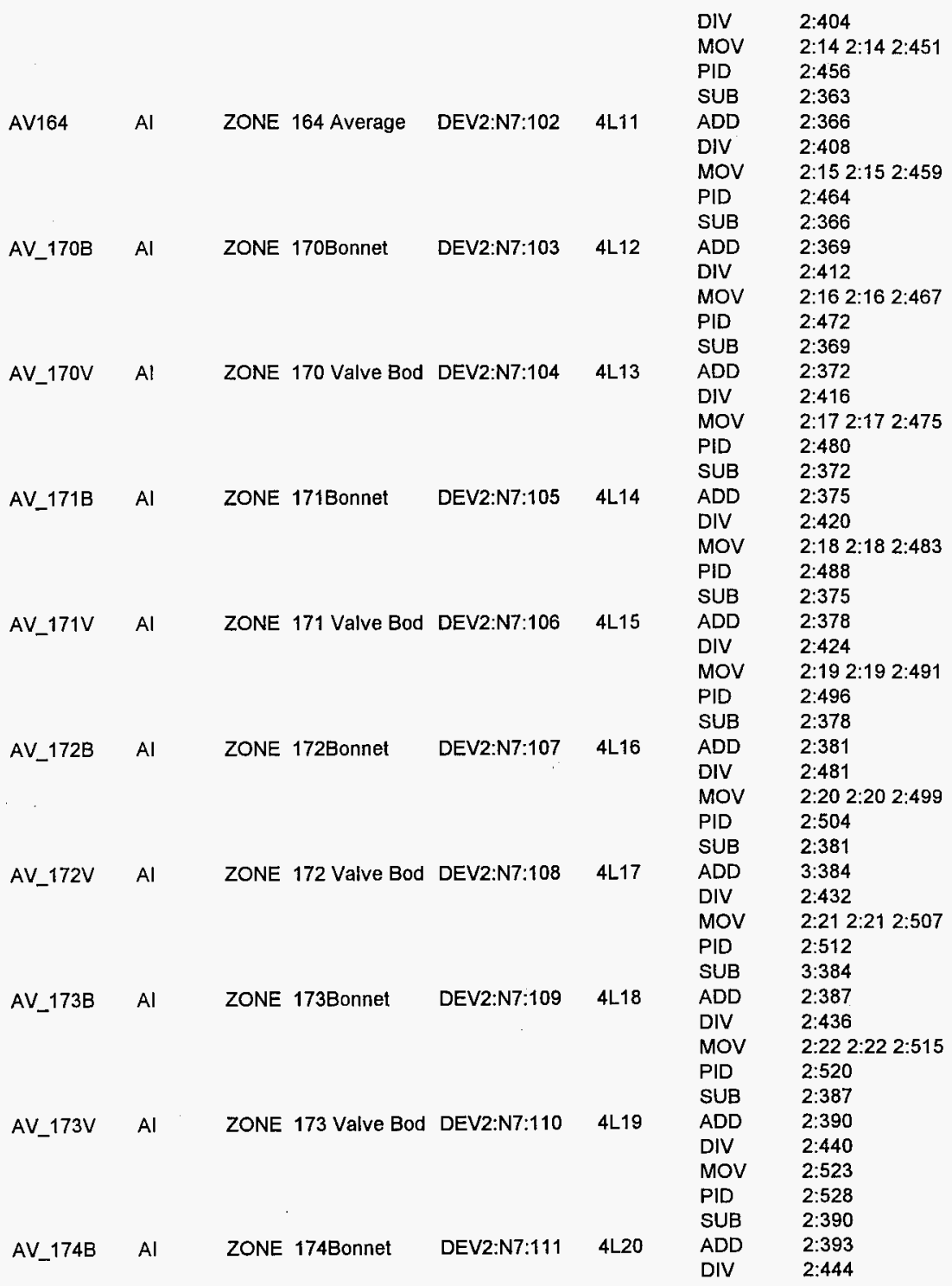




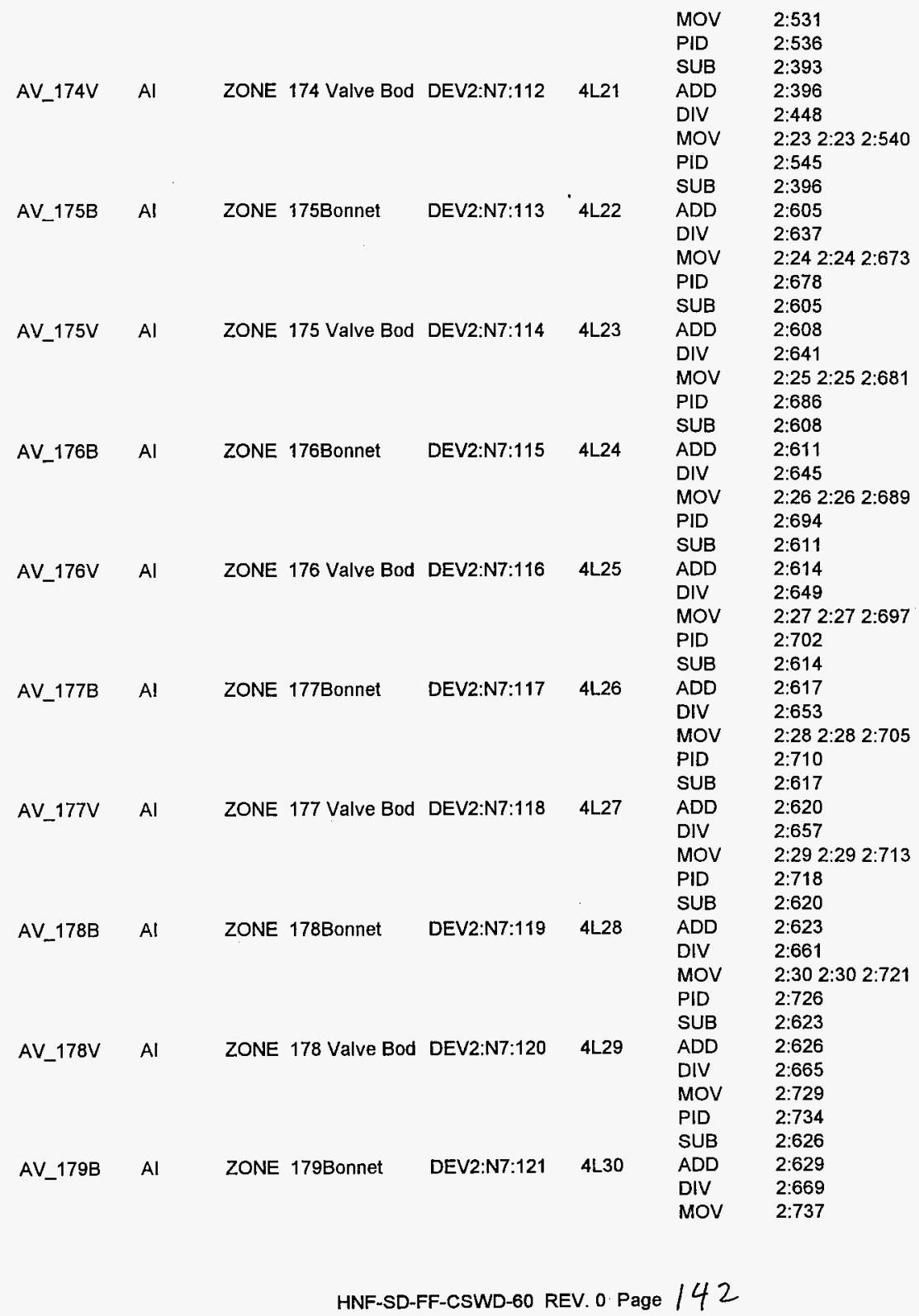




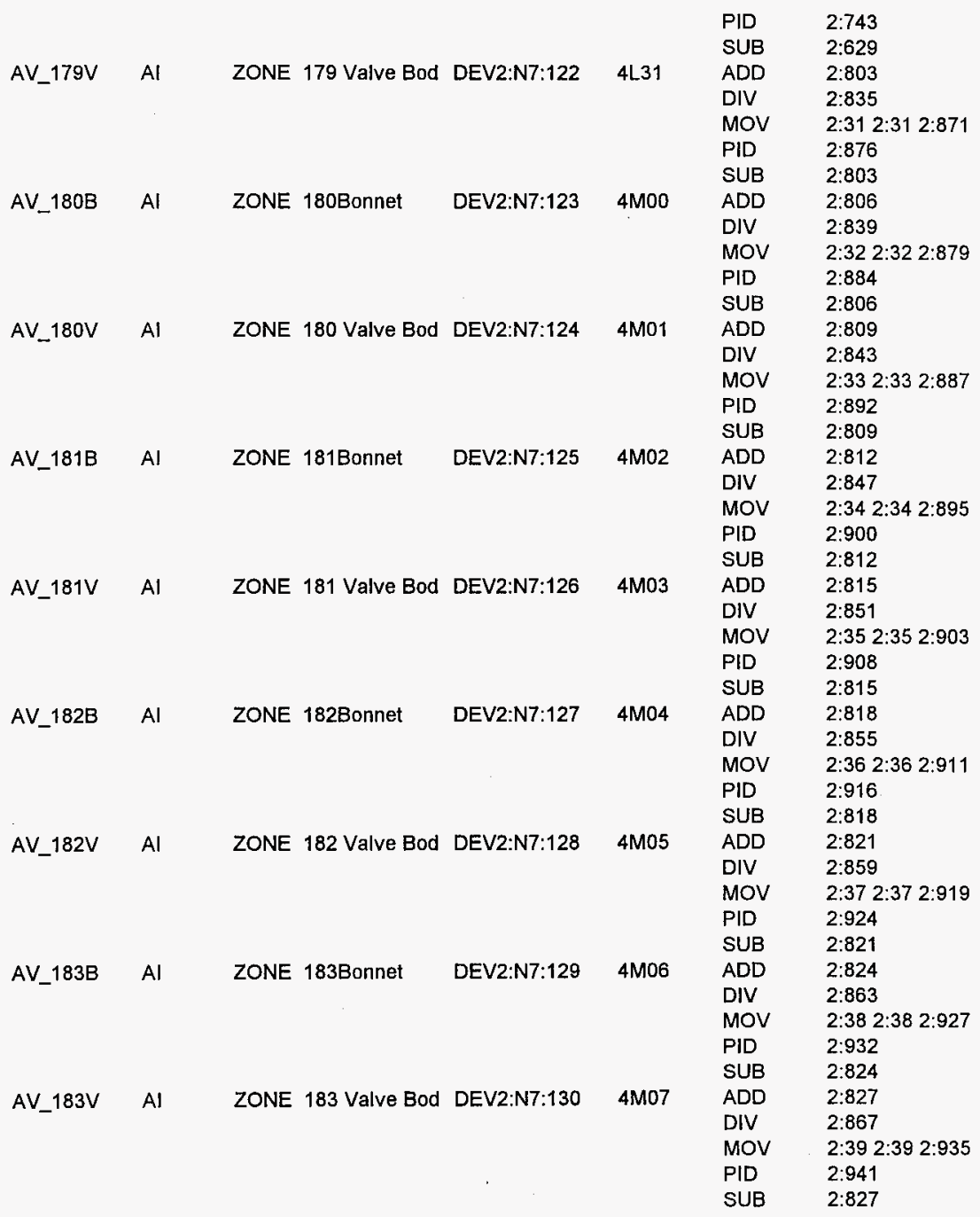




\begin{tabular}{|c|c|c|c|c|c|}
\hline SETRD143 & Al & SET POINT READBA & DEV2:N7:131 & MOV & $2: 219$ \\
\hline SETRD144 & Al & SET POINT READBA & DEV2:N7:132 & MOV & $2: 227$ \\
\hline SETRD150 & Al & SET POINT READBA & DEV2:N7:133 & MOV & $2: 235$ \\
\hline SETRD151 & Al & SET POINT READBA & DEV2:N7:134 & MOV & $2: 243$ \\
\hline SETRD152 & Al & SET POINT READBA & DEV2:N7:135 & MOV & $2: 251$ \\
\hline SETRD153 & Al & SET POINT READBA & DEV2:N7:136 & MOV & $2: 259$ \\
\hline SETRD154 & Al & SET POINT READBA & DEV2:N7:137 & MOV & $2: 267$ \\
\hline SETRD160 & Al & SET POINT READBA & DEV2:N7:138 & MOV & $2: 275$ \\
\hline SETRD161 & Al & SET POINT READBA & DEV2:N7:139 & MOV & $2: 283$ \\
\hline SETRD162 & $\mathrm{Al}$ & SET POINT READBA & DEV2:N7:140 & MOV & $2: 292$ \\
\hline SETRD163 & Al & SET POINT READBA & DEV2:N7:141 & MOV & $2: 455$ \\
\hline SETRD164 & Al & SET POINT READBA & DEV2:N7:142 & MOV & $2: 463$ \\
\hline SETRD170B & Al & SET POINT READBA & DEV2:N7:143 & MOV & $2: 471$ \\
\hline SETRD170V & Al & SET POINT READBA & DEV2:N7:144 & MOV & $2: 479$ \\
\hline SETRD171B & Al & SET POINT READBA & DEV2:N7:145 & MOV & $2: 487$ \\
\hline SETRD171V & Al & SET POINT READBA & DEV2:N7:146 & MOV & $2: 495$ \\
\hline SETRD172B & Al & SET POINT READBA & DEV2:N7:147 & MOV & $2: 503$ \\
\hline SETRD172V & Al & SET POINT READBA & DEV2:N7:148 & MOV & $2: 511$ \\
\hline SETRD173B & Al & SET POINT READBA & DEV2:N7:149 & MOV & $2: 519$ \\
\hline SETRD173V & Al & SET POINT READBA & DEV2:N7:150 & MOV & $2: 527$ \\
\hline SETRD174B & Al & SET POINT READBA & DEV2:N7:151 & MOV & $2: 535$ \\
\hline SETRD174V & Al & SET POINT READBA & DEV2:N7:152 & MOV & $2: 544$ \\
\hline SETRD175B & Al & SET POINT READBA & DEV2:N7:153 & MOV & $2: 677$ \\
\hline SETRD175V & Al & SET POINT READBA & DEV2:N7:154 & MOV & $2: 685$ \\
\hline SETRD176B & $\mathrm{Al}$ & SET POINT READBA & DEV2:N7:155 & MOV & $2: 693$ \\
\hline SETRD176V & $\mathrm{Al}$ & SET POINT READBA & DEV2:N7:156 & MOV & $2: 701$ \\
\hline SETRD177B & $\mathrm{Al}$ & SET POINT READBA & DEV2:N7:157 & MOV & $2: 709$ \\
\hline SETRD177V & Al & SET POINT READBA & DEV2:N7:158 & MOV & $2: 717$ \\
\hline SETRD178B & Al & SET POINT READBA & DEV2:N7:159 & MOV & $2: 725$ \\
\hline SETRD178V & $\mathrm{Al}$ & SET POINT READBA & DEV2:N7:160 & MOV & $2: 733$ \\
\hline SETRD179B & Al & SET POINT READBA & DEV2:N7:161 & MOV & $2: 742$ \\
\hline SETRD179V & Al & SET POINT READBA & DEV2:N7:162 & MOV & $2: 875$ \\
\hline SETRD180B & Al & SET POINT READBA & DEV2:N7:163 & MOV & $2: 833$ \\
\hline SETRD180V & $\mathrm{Al}$ & SET POINT READBA & DEV2:N7:164 & MOV & $2: 891$ \\
\hline SETRD181B & $\mathrm{Al}$ & SET POINT READBA & DEV2:N7:165 & MOV & $2: 899$ \\
\hline SETRD181V & Al & SET POINT READBA & DEV2:N7:166 & MOV & $2: 90$ \\
\hline SETRD182B & $\mathrm{Al}$ & SET POINT READBA & DEV2:N7:167 & MOV & $2: 915$ \\
\hline SETRD182V & Al & SET POINT READBA & DEV2:N7:168 & MOV & $2: 923$ \\
\hline SETRD183B & $\mathrm{Al}$ & SET POINT READBA & DEV2:N7:169 & MOV & $2: 931$ \\
\hline SETRD183V & Al & SET POINT READBA & DEV2:N7:170 & MOV & $2: 94$ \\
\hline
\end{tabular}




\begin{tabular}{|c|c|c|c|c|c|c|}
\hline D143A & DA & T/C Value Out of Ran & DEV2:N7:171/0 & 3D0 & $\begin{array}{l}-] /[- \\
-()-\end{array}$ & $\begin{array}{l}2: 170 \\
2: 134\end{array}$ \\
\hline \multirow[t]{2}{*}{ D143B } & DA & T/C Value Out of Ran & DEV2:N7:171/1 & 3D3 & $-] /[-$ & $2: 171$ \\
\hline & & & & & -()$-$ & $2: 135$ \\
\hline \multirow[t]{2}{*}{ D144A } & DA & T/C Value Out of Ran & DEV2:N7:171/2 & 3D6 & $-] /[-$ & $2: 174$ \\
\hline & & & & & & $2: 137$ \\
\hline \multirow[t]{2}{*}{ D144B } & DA & $T / C$ Value Out of Ran & DEV2:N7:171/3 & 3D9 & $-] /[-$ & $2: 175$ \\
\hline & & & & & -() & $2: 138$ \\
\hline \multirow[t]{2}{*}{ D150A } & DA & T/C Value Out of Ran & DEV2:N7:171/4 & $3 E 0$ & $-] /[-$ & $2: 178$ \\
\hline & & & & & & $2: 140$ \\
\hline \multirow[t]{2}{*}{ D150B } & $\mathrm{DA}$ & T/C Value Out of Ran & DEV2:N7:171/5 & $3 E 3$ & $-] /[-$ & $2: 179$ \\
\hline & & & & & & $2: 141$ \\
\hline \multirow[t]{2}{*}{ D150C } & DA & T/C Value Out of Ran & DEV2:N7:171/6 & $3 \mathrm{E} 6$ & $-] /[-$ & $2: 180$ \\
\hline & & & & & & $2: 142$ \\
\hline \multirow[t]{2}{*}{ D151A } & DA & T/C Value Out of Ran & DEV2:N7:171/7 & 3E9 & $-1 / /[-$ & $2: 183$ \\
\hline & & & & & , & $2: 144$ \\
\hline D151B & DA & T/C Value Out of Ran & DEV2:N7:171/8 & $3 F 0$ & $-j /$ & $2: 184$ \\
\hline \multirow{2}{*}{ D151C } & DA & T/C Value Out of Ran & DEV2:N7:171/9 & $3 F_{3}$ & $-1 /$ & $2: 185$ \\
\hline & & & & & -()$-$ & $2: 146$ \\
\hline \multirow[t]{2}{*}{ D152A } & DA & $T / C$ Value Out of Ran & DEV2:N7:171/10 & $3 F 6$ & $-] /[-$ & $2: 188$ \\
\hline & & & & & -()$-$ & $2: 148$ \\
\hline \multirow[t]{2}{*}{ D152B } & DA & T/C Value Out of Ran & DEV2:N7:171/11 & $3 \mathrm{Fg}$ & $-] /[-$ & $2: 189$ \\
\hline & & & & & -()$-$ & $2: 149$ \\
\hline \multirow[t]{2}{*}{ D153A } & DA & T/C Value Out of Ran & DEV2:N7:171/12 & $3 G 0$ & $-] /[-$ & $2: 192$ \\
\hline & & & & & -()$-$ & $2: 151$ \\
\hline \multirow[t]{2}{*}{ D153B } & DA & T/C Value Out of Ran & DEV2:N7:171/13 & $3 G 3$ & $-] /[-$ & $2: 193$ \\
\hline & & & & & -()$-$ & $2: 152$ \\
\hline \multirow[t]{2}{*}{ D154A } & DA & $T / C$ Value Out of Ran & DEV2:N7:171/14 & $3 G 6$ & $-] /[-$ & $2: 196$ \\
\hline & & & & & -() & $2: 154$ \\
\hline \multirow[t]{2}{*}{ D154B } & DA & T/C Value Out of Ran & DEV2:N7:171/15 & $3 \mathrm{G} 9$ & $-] /[-$ & $2: 197$ \\
\hline & & & & & -() & $2: 155$ \\
\hline \multirow[t]{2}{*}{ D160A } & DA & T/C Value Out of Ran & DEV2:N7:172/0 & $3 \mathrm{H} 0$ & $-] /[-$ & $2: 200$ \\
\hline & & & & & -()$-$ & $2: 157$ \\
\hline \multirow[t]{2}{*}{ D160B } & DA & T/C Value Out of Ran & DEV2:N7:172/1 & $3 \mathrm{H} 3$ & $-] /[-$ & $2: 201$ \\
\hline & & & & & -()$-$ & $2: 158$ \\
\hline \multirow[t]{2}{*}{ D160C } & DA & T/C Value Out of Ran & DEV2:N7:172/2 & $3 \mathrm{H} 6$ & $-] /[-$ & $2: 202$ \\
\hline & & & & & -()$-$ & $2: 159$ \\
\hline \multirow[t]{2}{*}{ D161A } & DA & T/C Value Out of Ran & DEV2:N7:172/3 & $3 \mathrm{H} 9$ & $-] /[-$ & $2: 205$ \\
\hline & & & & & & $2: 161$ \\
\hline \multirow[t]{2}{*}{ D161B } & DA & $\mathrm{T} / \mathrm{C}$ Value Out of Ran & DEV2:N7:172/4 & 310 & $-] /[-$ & $2: 206$ \\
\hline & & & & & -()$-$ & $2: 162$ \\
\hline D161C & DA & $T / C$ Value Out of Ran & DEV2:N7:172/5 & 313 & $-] /[-$ & $2: 207$ \\
\hline & & & & & -() & $2: 163$ \\
\hline D162A & DA & T/C Value Out of Ran & DEV2:N7:172/6 & 316 & $-] /[-$ & $2: 210$ \\
\hline & & & & & -()$-$ & $2: 165$ \\
\hline D162B & DA & $\mathrm{T} / \mathrm{C}$ Value Out of Ran & DEV2:N7:172/7 & 319 & $-] / 4-$ & $2: 211$ \\
\hline & & & & & -() & $2: 166$ \\
\hline D163A & DA & T/C Value Out of Ran & DEV2:N7:172/8 & 3J0 & $-] /[-$ & $2: 402$ \\
\hline & & & & & -()$-$ & $2: 364$ \\
\hline D163B & DA & T/C Value Out of Ran & DEV2:N7:172/9 & $3 \mathrm{~J} 3$ & $-] /[-$ & $2: 403$ \\
\hline
\end{tabular}




\begin{tabular}{|c|c|c|c|c|c|c|}
\hline \multirow{3}{*}{ D164A } & \multirow{3}{*}{$\mathrm{DA}$} & \multirow{3}{*}{ T/C Value Out of Ran } & \multirow{3}{*}{ DEV2:N7:172/10 } & \multirow{3}{*}{ 3.J6 } & \multirow{2}{*}{$\begin{array}{l}-()- \\
-] /[-\end{array}$} & \multirow{2}{*}{$\begin{array}{l}2: 365 \\
2: 406\end{array}$} \\
\hline & & & & & & \\
\hline & & & & & -()$-$ & $2: 367$ \\
\hline \multirow[t]{2}{*}{ D164B } & DA & T/C Value Out of Ran & DEV2:N7:172/11 & 3. 9 & $-] /[-$ & $2: 407$ \\
\hline & & & & & -()$-$ & $2: 368$ \\
\hline \multirow{2}{*}{ D170B1 } & DA & T/C Value Out of Ran & DEV2:N7:172/12 & $3 \mathrm{KO}$ & $-1 /[-$ & $2: 410$ \\
\hline & & & & & -()$-$ & $2: 370$ \\
\hline \multirow[t]{2}{*}{ D170B2 } & DA & $T / C$ Value Out of Ran & DEV2:N7:172/13 & $3 K 3$ & $-] /[-$ & $2: 411$ \\
\hline & & & & & -() & $2: 371$ \\
\hline \multirow[t]{2}{*}{ D170V1 } & DA & T/C Value Out of Ran & DEV2:N7:172/14 & $3 \mathrm{~K} 6$ & $-] /[-$ & $2: 414$ \\
\hline & & & & & & $2: 373$ \\
\hline \multirow[t]{2}{*}{ D170V2 } & DA & T/C Value Out of Ran & DEV2:N7:172/15 & $3 \mathrm{~K} 9$ & $-] /[-$ & $2: 415$ \\
\hline & & & & & & $2: 374$ \\
\hline \multirow[t]{2}{*}{ D171B1 } & DA & T/C Value Out of Ran & DEV2:N7:173/0 & 3LO & $-] /[-$ & $2: 418$ \\
\hline & & & & & & $2: 376$ \\
\hline \multirow[t]{2}{*}{ D171B2 } & DA & $\mathrm{T} / \mathrm{C}$ Value Out of Ran & DEV2:N7:173/1 & 3L3 & $-[/[-$ & $2: 419$ \\
\hline & & & & & i & $2: 377$ \\
\hline \multirow[t]{2}{*}{ D171V1 } & DA & $\mathrm{T} / \mathrm{C}$ Value Out of Ran & DEV2:N7:173/2 & 3L6 & $-] /[-$ & $2: 422$ \\
\hline & & & & & -()$-$ & $2: 379$ \\
\hline \multirow[t]{2}{*}{ D171V2 } & DA & T/C Value Out of Ran & DEV2:N7:173/3 & 3L9 & $-] /[-$ & $2: 423$ \\
\hline & & & & & -()$-$ & $2: 380$ \\
\hline \multirow[t]{2}{*}{ D172B1 } & DA & T/C Value Out of Ran & DEV2:N7:173/4 & $3 \mathrm{MO}$ & $-] /[-$ & $2: 426$ \\
\hline & & & & & -()$-$ & $2: 382$ \\
\hline \multirow[t]{2}{*}{$\mathrm{D} 172 \mathrm{~B} 2$} & DA & $\mathrm{T} / \mathrm{C}$ Value Out of Ran & DEV2:N7:173/5 & $3 \mathrm{M} 3$ & $-] /[-$ & $2: 427$ \\
\hline & & & & & & $2: 383$ \\
\hline \multirow[t]{2}{*}{ D172V1 } & DA & T/C Value Out of Ran & DEV2:N7:173/6 & $3 \mathrm{M} 6$ & $-] /[-$ & $2: 430$ \\
\hline & & & & & & $2: 385$ \\
\hline \multirow[t]{2}{*}{ D172V2 } & DA & T/C Value Out of Ran & DEV2:N7:173/7 & $3 \mathrm{M} 9$ & $-] /[-$ & $2: 431$ \\
\hline & & & & & & $2: 386$ \\
\hline D173B1 & DA & T/C Value Out of Ran & DEV2:N7:173/8 & $4 A 0$ & $-] /[-$ & $2: 434$ \\
\hline & & & & & -()$-$ & $2: 388$ \\
\hline D173B2 & DA & $T / C$ Value Out of Ran & DEV2:N7:173/9 & $4 A 3$ & $-3 / 1-$ & $2: 435$ \\
\hline & & & & & -()$-$ & $2: 389$ \\
\hline D173V1 & DA & T/C Value Out of Ran & DEV2:N7:173/10 & $4 A 6$ & $-] / 5-$ & $2: 438$ \\
\hline & & & & & -()$-$ & $2: 391$ \\
\hline D173V2 & DA & T/C Value Out of Ran & DEV2:N7:173/11 & $4 A 9$ & $-] /[-$ & $2: 439$ \\
\hline & & & & & -() & $2: 392$ \\
\hline D174B1 & DA & $T / C$ Value Out of Ran & DEV2:N7:173/12 & $4 \mathrm{~B} 0$ & $-] / 4-$ & $2: 442$ \\
\hline & & & & & -()$-$ & $2: 394$ \\
\hline $\mathrm{D} 174 \mathrm{~B} 2$ & DA & $\mathrm{T} / \mathrm{C}$ Value Out of Ran & DEV2:N7:173/13 & 4B3 & $-] /[-$ & $2: 443$ \\
\hline & & & & & -()$-$ & $2: 395$ \\
\hline D174V1 & DA & T/C Value Out of Ran & DEV2:N7:173/14 & $4 \mathrm{~B} 6$ & $-] /[-$ & $2: 446$ \\
\hline & & & & & -() & $2: 397$ \\
\hline D174V2 & DA & $T / C$ Value Out of Ran & DEV2:N7:173/15 & 4B9 & $-] /[-$ & $2: 447$ \\
\hline & & & & & -()$-$ & $2: 398$ \\
\hline $\mathrm{D} 175 \mathrm{~B} 1$ & DA & $T / C$ Value Out of Ran & DEV2:N7:174/0 & $4 \mathrm{CO}$ & $-] /[-$ & $2: 635$ \\
\hline & & & & & $-(1)$ & $2: 606$ \\
\hline D175B2 & DA & T/C Value Out of Ran & DEV2:N7:174/1 & $4 \mathrm{C} 3$ & $-] /[-$ & $2: 636$ \\
\hline & & & & & $-(j)$ & $2: 607$ \\
\hline D175V1 & DA & T/C Value Out of Ran & DEV2:N7:174/2 & $4 \mathrm{C} 6$ & $-] /[-$ & $2: 639$ \\
\hline & & & & & -()$-$ & $2: 609$ \\
\hline
\end{tabular}




\begin{tabular}{|c|c|c|c|c|c|c|}
\hline D175V2 & DA & T/C Value Out of Ran & DEV2:N7:174/3 & $4 C 9$ & $\begin{array}{l}-] /[- \\
-()-\end{array}$ & $\begin{array}{l}2: 640 \\
2: 610\end{array}$ \\
\hline D176B1 & DA & $T / C$ Value Out of Ran & DEV2:N7:174/4 & 4DO & $\begin{array}{l}-1 /[- \\
-()-\end{array}$ & $\begin{array}{l}2: 643 \\
2: 612\end{array}$ \\
\hline D176B2 & DA & $T / C$ Value Out of Ran & DEV2:N7:174/5 & 4D3 & $\begin{array}{l}-] /[- \\
-()-\end{array}$ & $\begin{array}{l}2: 644 \\
2: 613\end{array}$ \\
\hline D176V1 & DA & T/C Value Out of Ran & DEV2:N7:174/6 & 4D6 & $\begin{array}{l}-1 /[- \\
-()-\end{array}$ & $\begin{array}{l}2: 647 \\
2: 615\end{array}$ \\
\hline D176V2 & DA & T/C Value Out of Ran & DEV2:N7:174/7 & 409 & $\begin{array}{l}-] /[- \\
-()-\end{array}$ & $\begin{array}{l}2: 648 \\
2: 616\end{array}$ \\
\hline D177B1 & $\mathrm{DA}$ & $\mathrm{T} / \mathrm{C}$ Value Out of Ran & DEV2:N7:174/8 & 4EO & $\begin{array}{l}-] /[- \\
-()-\end{array}$ & $\begin{array}{l}2: 651 \\
2: 618\end{array}$ \\
\hline D177B2 & DA & $T / C$ Value Out of Ran & DEV2:N7:174/9 & 4E3 & $\begin{array}{l}-] /[- \\
-()\end{array}$ & $\begin{array}{l}2: 652 \\
2: 619\end{array}$ \\
\hline D177V1 & DA & T/C Value Out of Ran & DEV2:N7:174/10 & 4E6 & $\begin{array}{l}-] /[- \\
-()-\end{array}$ & $\begin{array}{l}2: 655 \\
2: 621\end{array}$ \\
\hline D177V2 & DA & T/C Value Out of Ran & DEV2:N7:174/11 & 4E9 & $\begin{array}{l}-] /[- \\
-()-\end{array}$ & $\begin{array}{l}2: 656 \\
2: 622\end{array}$ \\
\hline D178B1 & DA & $T / C$ Value Out of Ran & DEV2:N7:174/12 & 4FO & $\begin{array}{l}-] /[- \\
-()-\end{array}$ & $\begin{array}{l}2: 659 \\
2: 624\end{array}$ \\
\hline D178B2 & DA & T/C Value Out of Ran & DEV2:N7:174/13 & $4 \mathrm{~F} 3$ & $\begin{array}{l}-] /[- \\
-()-\end{array}$ & $\begin{array}{l}2: 660 \\
2: 625\end{array}$ \\
\hline D178V1 & DA & T/C Value Out of Ran & DEV2:N7:174/14 & 4F6 & $\begin{array}{l}-] /[- \\
-()-\end{array}$ & $\begin{array}{l}2: 663 \\
2: 627\end{array}$ \\
\hline D178V2 & DA & T/C Value Out of Ran & DEV2:N7:174/15 & $4 \mathrm{Fg}$ & $\begin{array}{l}-] /[- \\
-()-\end{array}$ & $\begin{array}{l}2: 664 \\
2: 628\end{array}$ \\
\hline D17981 & DA & $\mathrm{T} / \mathrm{C}$ Value Out of Ran & DEV2:N7:175/0 & $4 G 0$ & $\begin{array}{l}-] /[- \\
-()-\end{array}$ & $\begin{array}{l}2: 667 \\
2: 630\end{array}$ \\
\hline D179B2 & DA & T/C Value Out of Ran & DEV2:N7:175/1 & $4 \mathrm{G} 3$ & $\begin{array}{l}-] /[- \\
-()-\end{array}$ & $\begin{array}{l}2: 668 \\
2: 631\end{array}$ \\
\hline D179V1 & DA & T/C Value Out of Ran & DEV2:N7:175/2 & $4 G 6$ & $\begin{array}{l}-] /[- \\
-()-\end{array}$ & $\begin{array}{l}2: 833 \\
2: 804\end{array}$ \\
\hline D179V2 & $D A$ & T/C Value Out of Ran & DEV2:N7:175/3 & 4G9 & $\begin{array}{l}-] /[- \\
-()-\end{array}$ & $\begin{array}{l}2: 834 \\
2: 805\end{array}$ \\
\hline D180B1 & DA & T/C Value Out of Ran & DEV2:N7:175/4 & $4 \mathrm{HO}$ & $\begin{array}{l}-] /[- \\
-()-\end{array}$ & $\begin{array}{l}2: 837 \\
2: 807\end{array}$ \\
\hline D180B2 & DA & $\mathrm{T} / \mathrm{C}$ Value Out of Ran & DEV2:N7:175/5 & $4 \mathrm{H} 3$ & $\begin{array}{l}-] /[- \\
-()-\end{array}$ & $\begin{array}{l}2: 838 \\
2: 808\end{array}$ \\
\hline D180V1 & DA & T/C Value Out of Ran & DEV2:N7:175/6 & $4 H 6$ & $\begin{array}{l}-] /[- \\
-()-\end{array}$ & $\begin{array}{l}2: 841 \\
2: 810\end{array}$ \\
\hline D180V2 & DA & T/C Value Out of Ran & DEV2:N7:175/7 & $4 \mathrm{H} 9$ & $\begin{array}{l}-] /[- \\
-()-\end{array}$ & $\begin{array}{l}2: 842 \\
2: 811\end{array}$ \\
\hline D181B1 & DA & T/C Value Out of Ran & DEV2:N7:175/8 & 410 & $\begin{array}{l}-] /[- \\
-()-\end{array}$ & $\begin{array}{l}2: 845 \\
2: 813\end{array}$ \\
\hline D181B2 & DA & T/C Value Out of Ran & DEV2:N7:175/9 & 413 & $\begin{array}{l}-] /[- \\
-()-\end{array}$ & $\begin{array}{l}2: 846 \\
2: 814\end{array}$ \\
\hline D181V1 & $\mathrm{DA}$ & T/C Value Out of Ran & DEV2:N7:175/10 & 416 & $\begin{array}{l}-] /[- \\
-()\end{array}$ & $\begin{array}{l}2: 849 \\
2: 816\end{array}$ \\
\hline D181V2 & DA & $T / C$ Value Out of Ran & DEV2:N7:175/11 & 419 & $\begin{array}{l}-] /[- \\
-()-\end{array}$ & $\begin{array}{l}2: 850 \\
2: 817\end{array}$ \\
\hline D182B1 & DA & T/C Value Out of Ran & DEV2:N7:175/12 & 4J0 & $-] /[-$ & $2: 853$ \\
\hline
\end{tabular}


Tag Name Tag Type Description

PLC Device PLC 1/O Ladder Logic Rungs

\begin{tabular}{|c|c|c|c|c|c|c|}
\hline & & & & & -()$-$ & $2: 819$ \\
\hline \multirow[t]{2}{*}{ D182B2 } & DA & $\mathrm{T} / \mathrm{C}$ Value Out of Ran & DEV2:N7:175/13 & $4 \mathrm{~J} 3$ & $-1 /[-$ & $2: 854$ \\
\hline & & & & & -()$-$ & $2: 820$ \\
\hline \multirow[t]{2}{*}{ D182V1 } & DA & T/C Value Out of Ran & DEV2:N7:175/14 & $4 \sqrt{ } 6$ & $-1 /[-$ & $2: 857$ \\
\hline & & & & & -()$-$ & $2: 822$ \\
\hline \multirow[t]{2}{*}{$\mathrm{D} 182 \mathrm{~V} 2$} & DA & T/C Value Out of Ran & DEV2:N7:175/15 & $4 \mathrm{~J} 9$ & $-1 /\left[\left[^{-}\right.\right.$ & $2: 858$ \\
\hline & & & & & -()$-$ & $2: 823$ \\
\hline \multirow[t]{2}{*}{ D183B1 } & DA & $\mathrm{T} / \mathrm{C}$ Value Out of Ran & DEV2:N7:176/0 & $4 \mathrm{KO}$ & $-1 /[-$ & $2: 861$ \\
\hline & & & & & -( & $2: 825$ \\
\hline \multirow[t]{2}{*}{ D183B2 } & DA & $\mathrm{T} / \mathrm{C}$ Value Out of Ran & DEV2:N7:176/1 & $4 \mathrm{~K} 3$ & $-1 /[-$ & $2: 862$ \\
\hline & & & & & -()$-$ & $2: 826$ \\
\hline \multirow[t]{2}{*}{ D183V1 } & DA & $T / C$ Value Out of Ran & DEV2:N7:176/2 & $4 K 6$ & $-1 /[-$ & $2: 865$ \\
\hline & & & & & -()$-$ & $2: 828$ \\
\hline \multirow[t]{2}{*}{ D183V2 } & DA & $T / C$ Value Out of Ran & DEV2:N7:176/3 & $4 \mathrm{~K} 9$ & $-1 /[-$ & $2: 866$ \\
\hline & & & & & -()$-$ & $2: 829$ \\
\hline
\end{tabular}




\begin{tabular}{|c|c|c|c|c|c|c|}
\hline CV143 & $A O$ & TY-143 PID_CV & DEV2:N7:177 & 4LOO & PID & $2: 220$ \\
\hline CVRD143 & Al & CV READBĀCK & DEV2:N7:177 & 4L00 & $S C L$ & $2: 220$ \\
\hline CV144 & $\mathrm{AO}$ & TY-144 PID_CV & DEV2:N7:178 & 4L01 & PID & $2: 228$ \\
\hline CVRD144 & $\mathrm{Al}$ & CV READBĀCK & DEV2:N7:178 & 4L01 & SCL & $2: 228$ \\
\hline CV150 & $A O$ & TY-150 PID_CV & DEV2:N7:179 & 4L02 & PID & $2: 236$ \\
\hline CVRD150 & $\mathrm{Al}$ & CV READBĀCK & DEV2:N7:179 & 4LO2 & $S C L$ & $2: 236$ \\
\hline CV151 & $\mathrm{AO}$ & TY-151 PID_CV & DEV2:N7:180 & $4 \mathrm{LO}$ & PID & $2: 244$ \\
\hline CVRD151 & $\mathrm{Al}$ & CV READBĀCK & DEV2:N7:180 & 4L03 & SCL & $2: 244$ \\
\hline CV152 & $\mathrm{AO}$ & TY-152 PID_CV & DEV2:N7:181 & 4LO4 & PID & $2: 252$ \\
\hline CVRD152 & $\mathrm{Al}$ & CV READBĀCK & DEV2:N7:181 & 4L04 & $\mathrm{SCL}$ & $2: 252$ \\
\hline CV153 & $\mathrm{AO}$ & TY-153 PID_CV & DEV2:N7:182 & 4L05 & PID & $2: 260$ \\
\hline CVRD153 & $\mathrm{Al}$ & CV READBĀCK & DEV2:N7:182 & 4L05 & $S C L$ & $2: 260$ \\
\hline CV154 & AO & TY-154 PID_CV & DEV2:N7:183 & 4L06 & PID & $2: 268$ \\
\hline CVRD154 & $\mathrm{Al}$ & CV READBĀCK & DEV2:N7:183 & 4L06 & $\mathrm{SCL}$ & $2: 268$ \\
\hline CV160 & $\mathrm{AO}$ & TY-160 PID_CV & DEV2:N7:184 & 4L07 & PID & $2: 276$ \\
\hline CVRD160 & Al & CV READBACCK & DEV2:N7:184 & 4L07 & SCL & $2: 276$ \\
\hline CV161 & $\mathrm{AO}$ & TY-161 PID_CV & DEV2:N7:185 & 4L08 & PID & $2: 284$ \\
\hline CVRD161 & $\mathrm{Al}$ & CV READBACK & DEV2:N7:185 & 4L08 & SCL & 2:284 \\
\hline CV162 & $\mathrm{AO}$ & TY-162 PID_CV & DEV2:N7:186 & 4L09 & PID & $2: 293$ \\
\hline CVRD162 & $\mathrm{Al}$ & CV READBACK & DEV2:N7:186 & 4L09 & SCL & $2: 293$ \\
\hline CV163 & AO & TY-163 PID_CV & DEV2:N7:187 & $4 \mathrm{~L} 10$ & PID & $2: 456$ \\
\hline CVRD163 & AI & CV READBACK & DEV2:N7:187 & $4 L 10$ & $S C L$ & $2: 456$ \\
\hline CV164 & $\mathrm{AO}$ & TY-164 PID_CV & DEV2:N7:188 & 4L11 & PID & $2: 464$ \\
\hline CVRD164 & $\mathrm{Al}$ & CV READBĀCK & DEV2:N7:188 & $4 \mathrm{~L} 11$ & $\mathrm{SCL}$ & $2: 464$ \\
\hline CV $170 \mathrm{~B}$ & AO & TY-170B PID_CV & DEV2:N7:189 & $4 \mathrm{~L} 12$ & PID & $2: 472$ \\
\hline CVRD170B & Al & CV READBAC̄K & DEV2:N7:189 & $4 \mathrm{~L} 12$ & SCL & $2: 472$ \\
\hline CV170V & $\mathrm{AO}$ & TY-170V PID_CV & DEV2:N7:190 & $4 \mathrm{~L} 13$ & PID & $2: 480$ \\
\hline CVRD170V & $\mathrm{Al}$ & CV READBAC̄K & DEV2:N7:190 & $4 L 13$ & $\mathrm{SCL}$ & $2: 480$ \\
\hline CV171B & $\mathrm{AO}$ & TY-171B PID_CV & DEV2:N7:191 & 4L14 & PID & $2: 488$ \\
\hline CVRD171B & Al & CV READBAC̄K & DEV2:N7:191 & 4L14 & SCL & $2: 488$ \\
\hline CV171V & $\mathrm{AO}$ & TY-171VPID_CV & DEV2:N7:192 & 4L15 & PID & $2: 496$ \\
\hline CVRD171V & $\mathrm{Al}$ & CV READBAC̄K & DEV2:N7:192 & $4 L 15$ & SCL & $2: 496$ \\
\hline CV172B & $A O$ & TY-172B PID_CV & DEV2:N7:193 & $4 L 16$ & PID & $2: 504$ \\
\hline CVRD172B & $\mathrm{Al}$ & CV READBACKK & DEV2:N7:193 & $4 \mathrm{~L} 16$ & $\mathrm{SCL}$ & $2: 504$ \\
\hline CV172V & $\mathrm{AO}$ & TY-172V PID_CV & DEV2:N7:194 & $4 \mathrm{~L} 17$ & PID & $2: 512$ \\
\hline CVRD172V & $\mathrm{Al}$ & CV READBAC̄K & DEV2:N7:194 & 4L.17 & SCL. & $2: 512$ \\
\hline CV173B & $\mathrm{AO}$ & TY-173B PID_CV & DEV2:N7:195 & 4L18 & PID & $2: 520$ \\
\hline CVRD173B & $\mathrm{Al}$ & CV READBAC̄K & DEV2:N7:195 & 4L.18 & SCL & $2: 520$ \\
\hline CV173V & $\mathrm{AO}$ & TY-173V PID_CV & DEV2:N7:196 & $4 \mathrm{~L} 19$ & PID & $2: 528$ \\
\hline CVRD173V & $\mathrm{Al}$ & CV READBAC̄K & DEV2:N7:196 & 4L19 & $S C L$ & $2: 528$ \\
\hline CV174B & $\mathrm{AO}$ & TY-174B PID_CV & DEV2:N7:197 & $4 \mathrm{~L} 20$ & PID & $2: 536$ \\
\hline CVRD174B & $\mathrm{Al}$ & CV READBACK & DEV2:N7:197 & $4 \mathrm{~L} 20$ & $S C L$ & $2: 536$ \\
\hline CV174V & $\mathrm{AO}$ & TY-174V PID_CV & DEV2:N7:198 & $4 \mathrm{~L} 21$ & PID & $2: 545$ \\
\hline CVRD174V & $\mathrm{Al}$ & CV READBAC̄K & DEV2:N7:198 & $4 \mathrm{~L} 21$ & $\mathrm{SCL}$ & $2: 545$ \\
\hline CV175B & $\mathrm{AO}$ & TY-175B PID_CV & DEV2:N7:199 & $4 L 22$ & PID & $2: 678$ \\
\hline CVRD175B & Al & CV READBAC̄K & DEV2:N7:199 & $4 \mathrm{~L} 22$ & $\mathrm{SCL}$ & $2: 678$ \\
\hline CV175V & $A O$ & TY-175V PID_CV & DEV2:N7:200 & $4 \mathrm{~L} 23$ & PID & $2: 686$ \\
\hline CVRD175V & $\mathrm{Al}$ & CV READBACKK & DEV2:N7:200 & $4\llcorner 23$ & SCL & $2: 686$ \\
\hline CV176B & $A O$ & TY-176B PID_CV & DEV2:N7:201 & 4L24 & PID & $2: 694$ \\
\hline CVRD176B & $\mathrm{Al}$ & CV READBAC̄K & DEV2:N7:201 & $4\llcorner 24$ & $S C L$ & $2: 694$ \\
\hline CV176V & $\mathrm{AO}$ & TY-176V PID_CV & DEV2:N7:202 & $4\llcorner .25$ & PID & $2: 702$ \\
\hline
\end{tabular}




\begin{tabular}{|c|c|c|c|c|c|c|}
\hline CVRD176V & $\mathrm{Al}$ & CV READBACK & DEV2:N7:202 & $4 \mathrm{~L} 25$ & $\mathrm{SCL}$ & $2: 702$ \\
\hline CV177B & $A O$ & TY-177B PID_cV & DEV2:N7:203 & 4L26 & PID & $2: 710$ \\
\hline \multirow[t]{2}{*}{ CVRD177B } & $\mathrm{Al}$ & CV READBAC̄K & DEV2:N7:203 & $4 \mathrm{~L} 26$ & SCL & $2: 710$ \\
\hline & & & & & CLR & $2: 710$ \\
\hline CV177V & $\mathrm{AO}$ & TY-177V PID_CV & DEV2:N7:204 & $4 \operatorname{L} 27$ & PID & $2: 718$ \\
\hline \multirow[t]{2}{*}{ CVRD177V } & $\mathrm{Al}$ & CV READBAC̄K & DEV2:N7:204 & $4 \mathrm{~L} 27$ & SCL & $2: 718$ \\
\hline & & & & & CLR & $2: 718$ \\
\hline CV178B & $A O$ & TY-178B PID_cV & DEV2:N7:205 & $4 L 28$ & PID & $2: 726$ \\
\hline CVRD178B & $\mathrm{Al}$ & CV READBAC̄K & DEV2:N7:205 & $4 \mathrm{~L} 28$ & SCL & $2: 726$ \\
\hline CV178V & $A O$ & TY-178V PID_CV & DEV2:N7:206 & $4\llcorner 29$ & PID & $2: 734$ \\
\hline CVRD178V & Al & CV READBAC̄K & DEV2:N7:206 & $4\llcorner 29$ & $\mathrm{SCL}$ & $2: 734$ \\
\hline CV179B & $A O$ & TY-179B PID_CV & DEV2:N7:207 & 4L30 & PID & $2: 743$ \\
\hline \multirow[t]{2}{*}{ CVRD1798 } & $\mathrm{Al}$ & CV READBACK & DEV2:N7:207 & 4L30 & PID & $2: 743$ \\
\hline & & & & & CLR & $2: 743$ \\
\hline CV179V & $A O$ & TY-179V PID_CV & DEV2:N7:208 & 4L31 & PID & $2: 876$ \\
\hline CVRD179V & $\mathrm{Al}$ & CV READBACK & DEV2:N7:208 & 4L31 & SCL & $2: 876$ \\
\hline $\mathrm{CV} 180 \mathrm{~B}$ & $\mathrm{AO}$ & TY-180B PID_CV & DEV2:N7:209 & $4 \mathrm{M} 00$ & PID & $2: 884$ \\
\hline CVRD180B & Al & CV READBAC̄K & DEV2:N7:209 & $4 \mathrm{M} 00$ & $S C L$ & $2: 884$ \\
\hline CV180V & $A O$ & TY-180V PID_CV & DEV2:N7:210 & $4 \mathrm{M} 01$ & PID & $2: 892$ \\
\hline CVRD180V & $\mathrm{Al}$ & CV READBACK & DEV2:N7:210 & $4 \mathrm{M} 01$ & SCL & $2: 892$ \\
\hline CV181B & $A O$ & TY-181B PID_CV & DEV2:N7:211 & $4 \mathrm{M} 02$ & PID & $2: 900$ \\
\hline CVRD181B & $\mathrm{Al}$ & CV READBAC̄K & DEV2:N7:211 & $4 \mathrm{M} 02$ & SCL & $2: 900$ \\
\hline CV181V & $A O$ & TY-181V PID_CV & DEV2:N7:212 & $4 \mathrm{M} 03$ & PID & $2: 908$ \\
\hline CVRD181V & $\mathrm{Al}$ & CV READBACK & DEV2:N7:212 & $4 \mathrm{M} 03$ & SCL & $2: 908$ \\
\hline $\mathrm{CV} 182 \mathrm{~B}$ & $\mathrm{AO}$ & TY-182B PID_CV & DEV2:N7:213 & 4M04 & PID & $2: 916$ \\
\hline CVRD182B & $\mathrm{Al}$ & CV READBAC $K$ & DEV2:N7:213 & $4 \mathrm{MO4}$ & SCL & $2: 916$ \\
\hline CV182V & $A O$ & TY-182V PID_CV & DEV2:N7:214 & $4 M 05$ & PID & $2: 924$ \\
\hline CVRD182V & $\mathrm{Al}$ & CV READBAC̄K & DEV2:N7:214 & $4 \mathrm{MOS}$ & SCL & $2: 924$ \\
\hline CV183B & $\mathrm{AO}$ & TY-183B PID_CV & DEV2:N7:215 & $4 M 06$ & PID & $2: 932$ \\
\hline CVRD183B & $\mathrm{Al}$ & CV READBAC̄K & DEV2:N7:215 & $4 M 06$ & $S C L$ & $2: 932$ \\
\hline CV183V & $A O$ & TY-183V PID_CV & DEV2:N7:216 & $4 \mathrm{M07}$ & PID & $2: 941$ \\
\hline CVRD183V & $\mathrm{Al}$ & CV READBACK & DEV2:N7:216 & $4 \mathrm{M} 07$ & SCL & $2: 941$ \\
\hline
\end{tabular}


Tag Name Tag Type Description $\underline{\text { PLC Device }}$ PLC I/O Ladder Logic Rungs

\begin{tabular}{|c|c|c|c|c|c|c|}
\hline HA143A & DA & T/C Hardware Alarms & DEV2:N7:224/0 & 3D0 & $\begin{array}{l}-] /[- \\
-()-\end{array}$ & $\begin{array}{l}2: 1342: 170 \\
2: 107\end{array}$ \\
\hline HA143B & DA & T/C Hardware Alarms & DEV2:N7:224/1 & 3D3 & $\begin{array}{l}-] /[- \\
-()-\end{array}$ & $\begin{array}{l}2: 1352: 171 \\
2: 108\end{array}$ \\
\hline HA144A & DA & T/C Hardware Alarms & DEV2:N7:224/2 & 3D6 & $\begin{array}{l}-3 /[- \\
-()-\end{array}$ & $\begin{array}{l}2: 1372: 174 \\
2: 109\end{array}$ \\
\hline HA144B & DA & T/C Hardware Alarms & DEV2:N7:224/3 & 3D9 & $\begin{array}{l}-] /[- \\
-()-\end{array}$ & $\begin{array}{l}2: 1382: 175 \\
2: 110\end{array}$ \\
\hline HA150A & DA & T/C Hardware Alarms & DEV2:N7:224/4 & 3E0 & $\begin{array}{l}-] /[- \\
-()-\end{array}$ & $\begin{array}{l}2: 1402: 178 \\
2: 111\end{array}$ \\
\hline HA150B & DA & T/C Hardware Alarms & DEV2:N7:224/5 & $3 E 3$ & $\begin{array}{l}-] /[- \\
-()-\end{array}$ & $\begin{array}{l}2: 1412: 179 \\
2: 112\end{array}$ \\
\hline HA150C & DA & T/C Hardware Alarms & DEV2:N7:224/6 & $3 \mathrm{E} 6$ & $\begin{array}{l}-] /[- \\
-()-\end{array}$ & $\begin{array}{l}2: 1422: 180 \\
2: 113\end{array}$ \\
\hline HA151A & DA & T/C Hardware Alarms & DEV2:N7:224/7 & 3 E9 & $\begin{array}{l}-] /[- \\
-()-\end{array}$ & $\begin{array}{l}2: 1442: 183 \\
2: 114\end{array}$ \\
\hline HA151B & DA & T/C Hardware Alarms & DEV2:N7:224/8 & 3F0 & $\begin{array}{l}-] /[- \\
-()-\end{array}$ & $\begin{array}{l}2: 1452: 184 \\
2: 115\end{array}$ \\
\hline HA151C & DA & T/C Hardware Alarms & DEV2:N7:224/9 & $3 F 3$ & $\begin{array}{l}-] /[- \\
-()-\end{array}$ & $\begin{array}{l}2: 1462: 185 \\
2: 116\end{array}$ \\
\hline HA152A & DA & T/C Hardware Alarms & DEV2:N7:224/10 & $3 F 6$ & $\begin{array}{l}-] /[- \\
-()-\end{array}$ & $\begin{array}{l}2: 1482: 188 \\
2: 117\end{array}$ \\
\hline HA152B & DA & T/C Hardware Alarms & DEV2:N7:224/11 & $3 F 9$ & $\begin{array}{l}-] /[- \\
-()-\end{array}$ & $\begin{array}{l}2: 1492: 189 \\
2: 118\end{array}$ \\
\hline HA153A & DA & T/C Hardware Alarms & DEV2:N7:224/12 & $3 \mathrm{GO}$ & $\begin{array}{l}-] /[- \\
-()-\end{array}$ & $\begin{array}{l}2: 1512: 192 \\
2: 119\end{array}$ \\
\hline HA153B & DA & T/C Hardware Alarms & DEV2:N7:224/13 & $3 G 3$ & $\begin{array}{l}-1 /[- \\
-()-\end{array}$ & $\begin{array}{l}2: 152 \text { 2:193 } \\
2: 120\end{array}$ \\
\hline HA154A & DA & T/C Hardware Alarms & DEV2:N7:224/14 & $3 \mathrm{G} 6$ & $\begin{array}{l}-] /[- \\
-()-\end{array}$ & $\begin{array}{l}2: 1542: 196 \\
2: 121\end{array}$ \\
\hline HA154B & DA & T/C Hardware Alarms & DEV2:N7:224/15 & $3 G 9$ & $\begin{array}{l}-] /[- \\
-()-\end{array}$ & $\begin{array}{l}2: 1552: 197 \\
2: 122\end{array}$ \\
\hline HA160A & DA & T/C Hardware Alarms & DEV2:N7:225/0 & $3 \mathrm{H} 0$ & $\begin{array}{l}-] /[- \\
-()-\end{array}$ & $\begin{array}{l}2: 1572: 200 \\
2: 123\end{array}$ \\
\hline HA160B & DA & T/C Hardware Alarms & DEV2:N7:225/1 & $3 \mathrm{H} 3$ & $\begin{array}{l}-] /[- \\
-()-\end{array}$ & $\begin{array}{l}2: 158 \text { 2:201 } \\
2: 124\end{array}$ \\
\hline HA160C & DA & T/C Hardware Alarms & DEV2:N7:225/2 & $3 \mathrm{H} 6$ & $\begin{array}{l}-] /[- \\
-()-\end{array}$ & $\begin{array}{l}2: 1592: 202 \\
2: 125\end{array}$ \\
\hline HA161A & DA & T/C Hardware Alarms & DEV2:N7:225/3 & $3 \mathrm{H} 9$ & $\begin{array}{l}-] /[- \\
-()-\end{array}$ & $\begin{array}{l}2: 1612: 205 \\
2: 126\end{array}$ \\
\hline HA161B & DA & T/C Hardware Alarms & DEV2:N7:225/4 & 310 & $\begin{array}{l}-] / /- \\
-()-\end{array}$ & $\begin{array}{l}2: 1622: 206 \\
2: 127\end{array}$ \\
\hline HA161C & DA & T/C Hardware Alarms & DEV2:N7:225/5 & 313 & $\begin{array}{l}-] /[- \\
-()-\end{array}$ & $\begin{array}{l}2: 1632: 207 \\
2: 128\end{array}$ \\
\hline HA162A & DA & T/C Hardware Alarms & DEV2:N7:225/6 & 316 & $\begin{array}{l}-3 /[- \\
-()-\end{array}$ & $\begin{array}{l}2: 165 \text { 2:210 } \\
2: 129\end{array}$ \\
\hline HA162B & DA & T/C Hardware Alarms & DEV2:N7:225/7 & 319 & $\begin{array}{l}-] /[- \\
-()-\end{array}$ & $\begin{array}{l}2: 1662: 211 \\
2: 130\end{array}$ \\
\hline HA163A & DA & T/C Hardware Alarms & DEV2:N7:225/8 & $3 J 0$ & $\begin{array}{l}-] / /- \\
-()-\end{array}$ & $\begin{array}{l}2: 3642: 402 \\
2: 337\end{array}$ \\
\hline HA163B & DA & T/C Hardware Alarms & DEV2:N7:225/9 & $3 \mathrm{~J} 3$ & $-] /[-$ & $2: 3652: 403$ \\
\hline
\end{tabular}




\begin{tabular}{|c|c|c|c|c|c|c|}
\hline & & & & & -()$-$ & $2: 338$ \\
\hline HA164A & DA & T/C Hardware Alarms & DEV2:N7:225/10 & 3J6 & $\begin{array}{l}-] /[- \\
-()-\end{array}$ & $\begin{array}{l}2: 3672: 406 \\
2: 339\end{array}$ \\
\hline HA164B & DA & T/C Hardware Alarms & DEV2:N7:225/11 & 3J9 & $\begin{array}{l}-] /[- \\
-(0)\end{array}$ & $\begin{array}{l}2: 3682: 407 \\
2: 340\end{array}$ \\
\hline HA170B & DA & T/C Hardware Alarms & DEV2:N7:225/12 & $3 K 0$ & $\begin{array}{l}-] /[- \\
-()-\end{array}$ & $\begin{array}{l}2: 3702: 410 \\
2: 341\end{array}$ \\
\hline HA170B & DA & T/C Hardware Alarms & DEV2:N7:225/13 & $3 K 3$ & $\begin{array}{l}-] /[- \\
-()-\end{array}$ & $\begin{array}{l}2: 3712: 411 \\
2: 342\end{array}$ \\
\hline HA170V & DA & T/C Hardware Alarms & DEV2:N7:225/14 & $3 K 6$ & $\begin{array}{l}-] /[- \\
-()-\end{array}$ & $\begin{array}{l}2: 3732: 414 \\
2: 343\end{array}$ \\
\hline HA170V & $\mathrm{DA}$ & T/C Hardware Alarms & DEV2:N7:225/15 & $3 \mathrm{~K} 9$ & $\begin{array}{l}-1 /[- \\
-()-\end{array}$ & $\begin{array}{l}2: 3742: 415 \\
2: 344\end{array}$ \\
\hline HA171B & $\mathrm{DA}$ & T/C Hardware Alarms & DEV2:N7:226/0 & 3LO & $\begin{array}{l}-] /[- \\
-()-\end{array}$ & $\begin{array}{l}2: 3762: 418 \\
2: 345\end{array}$ \\
\hline HA171B & $\mathrm{DA}$ & T/C Hardware Alarms & DEV2:N7:226/1 & 3L.3 & $\begin{array}{l}-] /[- \\
-()\end{array}$ & $\begin{array}{l}2: 3772: 419 \\
2: 346\end{array}$ \\
\hline HA171V & $\mathrm{DA}$ & T/C Hardware Alarms & DEV2:N7:226/2 & 3L6 & $\begin{array}{l}-y /[- \\
-()-\end{array}$ & $\begin{array}{l}2: 3792: 422 \\
2: 347\end{array}$ \\
\hline HA171V & $\mathrm{DA}$ & T/C Hardware Alarms & DEV2:N7:226/3 & 3L9 & $\begin{array}{l}-] /[- \\
-()-\end{array}$ & $\begin{array}{l}2: 3802: 423 \\
2: 348\end{array}$ \\
\hline HA172B & DA & T/C Hardware Alarms & DEV2:N7:226/4 & $3 \mathrm{MO}$ & $\begin{array}{l}-] /[- \\
-() .\end{array}$ & $\begin{array}{l}2: 3822: 426 \\
2: 349\end{array}$ \\
\hline HA172B & $\mathrm{DA}$ & T/C Hardware Alarms & DEV2:N7:226/5 & $3 \mathrm{M} 3$ & $\begin{array}{l}-] /[- \\
-()\end{array}$ & $\begin{array}{l}2: 3832: 427 \\
2: 350\end{array}$ \\
\hline HA172V & $\mathrm{DA}$ & T/C Hardware Alarms & DEV2:N7:226/6 & $3 \mathrm{M} 6$ & $\begin{array}{l}-] /[- \\
-()-\end{array}$ & $\begin{array}{l}2: 385 \text { 2:430 } \\
2: 351\end{array}$ \\
\hline $\mathrm{HA172V}$ & DA & T/C Hardware Alarms & DEV2:N7:226/7 & 3M9 & $\begin{array}{l}-] /[- \\
-()-\end{array}$ & $\begin{array}{l}2: 3862: 431 \\
2: 352\end{array}$ \\
\hline HA173B & DA & T/C Hardware Alarms & DEV2:N7:226/8 & $4 A 0$ & $\begin{array}{l}-] /[- \\
-()-\end{array}$ & $\begin{array}{l}2: 388 \text { 2:434 } \\
2: 353\end{array}$ \\
\hline HA173B & DA & T/C Hardware Alarms & DEV2:N7:226/9 & $4 A 3$ & $\begin{array}{l}-] /[- \\
-()-\end{array}$ & $\begin{array}{l}2: 3892: 435 \\
2: 354\end{array}$ \\
\hline HA173V & $\mathrm{DA}$ & T/C Hardware Alarms & DEV2:N7:226/10 & $4 A 6$ & $\begin{array}{l}-] /[- \\
-()-\end{array}$ & $\begin{array}{l}\text { 2:391 2:438 } \\
2: 355\end{array}$ \\
\hline HA173V & DA & T/C Hardware Alarms & DEV2:N7:226/11 & $4 A 9$ & $\begin{array}{l}-] /[- \\
-()-\end{array}$ & $\begin{array}{l}2: 3922: 439 \\
2: 356\end{array}$ \\
\hline HA174B & DA & T/C Hardware Alarms & DEV2:N7:226/12 & 480 & $\begin{array}{l}-] /[- \\
-()-\end{array}$ & $\begin{array}{l}2: 3942: 442 \\
2: 357\end{array}$ \\
\hline HA174B & $\mathrm{DA}$ & T/C Hardware Alarms & DEV2:N7:226/13 & $4 \mathrm{~B} 3$ & $\begin{array}{l}-] /[- \\
-()-\end{array}$ & $\begin{array}{l}2: 3952: 443 \\
2: 358\end{array}$ \\
\hline HA174V & $\mathrm{DA}$ & T/C Hardware Alarms & DEV2:N7:226/14 & $4 \mathrm{~B} 6$ & $\begin{array}{l}-] /[- \\
-()-\end{array}$ & $\begin{array}{l}2: 3972: 446 \\
2: 359\end{array}$ \\
\hline HA174V & DA & T/C Hardware Alarms & DEV2:N7:226/15 & $4 \mathrm{Bg}$ & $\begin{array}{l}-] /[- \\
-()-\end{array}$ & $\begin{array}{l}2: 3982: 447 \\
2: 360\end{array}$ \\
\hline HA175B & DA & T/C Hardware Alarms & DEV2:N7:227/0 & $4 \mathrm{CO}$ & $\begin{array}{l}-] /[- \\
-()-\end{array}$ & $\begin{array}{l}2: 6062: 635 \\
2: 585\end{array}$ \\
\hline HA175B & DA & T/C Hardware Alarms & DEV2:N7:227/1 & $4 C 3$ & $\begin{array}{l}-] /[- \\
-()-\end{array}$ & $\begin{array}{l}2: 6072: 636 \\
2: 586\end{array}$ \\
\hline HA175V & DA & T/C Hardware Alarms & DEV2:N7:227/2 & $4 \mathrm{C} 6$ & $\begin{array}{l}-] /[- \\
-()-\end{array}$ & $\begin{array}{l}2: 6092: 639 \\
2: 587\end{array}$ \\
\hline
\end{tabular}


Tag Name Tag Type Description PLC Device PLC I/O Ladder Logic Rungs

\begin{tabular}{|c|c|c|c|c|c|c|}
\hline HA175V & $\mathrm{DA}$ & T/C Hardware Alarms & DEV2:N7:227/3 & $4 \mathrm{Cg}$ & $\begin{array}{l}-] /[- \\
-()-\end{array}$ & $\begin{array}{l}2: 6102: 640 \\
2: 588\end{array}$ \\
\hline HA176B & DA & T/C Hardware Alarms & DEV2:N7:227/4 & 4DO & $\begin{array}{l}-] /[- \\
-()-\end{array}$ & $\begin{array}{l}2: 6122: 643 \\
2: 589\end{array}$ \\
\hline HA176B & DA & T/C Hardware Alarms & DEV2:N7:227/5 & 4D3 & $\begin{array}{l}-j /[- \\
-()-\end{array}$ & $\begin{array}{l}2: 6132: 644 \\
2: 590\end{array}$ \\
\hline HA176V & DA & T/C Hardware Alarms & DEV2:N7:227/6 & 4D6 & $\begin{array}{l}-] /[- \\
-()-\end{array}$ & $\begin{array}{l}2: 6152: 647 \\
2: 591\end{array}$ \\
\hline HA176V & DA & T/C Hardware Alarms & DEV2:N7:227/7 & 4D9 & $\begin{array}{l}-] /[- \\
-()-\end{array}$ & $\begin{array}{l}2: 6162: 648 \\
2: 592\end{array}$ \\
\hline HA177B & DA & T/C Hardware Alarms & DEV2:N7:227/8 & 4E0 & $\begin{array}{l}-7 / 1- \\
-()-\end{array}$ & $\begin{array}{l}2: 6182: 651 \\
2: 593\end{array}$ \\
\hline HA177B & $\mathrm{DA}$ & T/C Hardware Alarms & DEV2:N7:227/9 & $4 \mathrm{E} 3$ & $\begin{array}{l}-] /[- \\
-()\end{array}$ & $\begin{array}{l}2: 6192: 652 \\
2: 594\end{array}$ \\
\hline HA177V & DA & T/C Hardware Alarms & DEV2:N7:227/10 & 4E6 & $\begin{array}{l}-1 /[- \\
-()-\end{array}$ & $\begin{array}{l}2: 6212: 655 \\
2: 595\end{array}$ \\
\hline HA177V & $\mathrm{DA}$ & T/C Hardware Alarms & DEV2:N7:227/11 & $4 E 9$ & $\begin{array}{l}-] /[- \\
-()-\end{array}$ & $\begin{array}{l}2: 6222: 656 \\
2: 596\end{array}$ \\
\hline HA178B & $\mathrm{DA}$ & T/C Hardware Alarms & DEV2:N7:227/12 & 4FO & $\begin{array}{l}-] /[- \\
-()-\end{array}$ & $\begin{array}{l}2: 6242: 659 \\
2: 597\end{array}$ \\
\hline HA178B & DA & T/C Hardware Alarms & DEV2:N7:227/13 & $4 \mathrm{~F} 3$ & $\begin{array}{l}-] /[- \\
-()-\end{array}$ & $\begin{array}{l}2: 6252: 660 \\
2: 598\end{array}$ \\
\hline HA178V & DA & T/C Hardware Alarms & DEV2:N7:227/14 & $4 \mathrm{~F} 6$ & $\begin{array}{l}-] /[- \\
-()-\end{array}$ & $\begin{array}{l}2: 6272: 663 \\
2: 599\end{array}$ \\
\hline HA178V & DA & T/C Hardware Alarms & DEV2:N7:227/15 & 4F9 & $\begin{array}{l}-] /[- \\
-()-\end{array}$ & $\begin{array}{l}2: 6282: 664 \\
2: 600\end{array}$ \\
\hline HA179B & DA & T/C Hardware Alarms & DEV2:N7:228/0 & $4 \mathrm{GO}$ & $\begin{array}{l}-] /[- \\
-()-\end{array}$ & $\begin{array}{l}2: 6302: 667 \\
2: 601\end{array}$ \\
\hline HA179B & DA & T/C Hardware Alarms & DEV2:N7:228/1 & $4 \mathrm{G} 3$ & $\begin{array}{l}-] /[- \\
-()-\end{array}$ & $\begin{array}{l}2: 6312: 668 \\
2: 602\end{array}$ \\
\hline HA179V & DA & T/C Hardware Alarms & DEV2:N7:228/2 & $4 \mathrm{G} 6$ & $\begin{array}{l}-] /[- \\
-()-\end{array}$ & $\begin{array}{l}2: 8042: 833 \\
2: 783\end{array}$ \\
\hline HA179V & DA & T/C Hardware Alarms & DEV2:N7:228/3 & $4 G 9$ & $\begin{array}{l}-] /[- \\
-()-\end{array}$ & $\begin{array}{l}2: 8052: 834 \\
2: 784\end{array}$ \\
\hline HA180B & DA & T/C Hardware Alarms & DEV2:N7:228/4 & $4 \mathrm{HO}$ & $\begin{array}{l}-] /[- \\
-()-\end{array}$ & $\begin{array}{l}2: 8072: 837 \\
2: 785\end{array}$ \\
\hline HA180B & DA & T/C Hardware Alarms & DEV2:N7:228/5 & $4 \mathrm{H} 3$ & $\begin{array}{l}-] /[- \\
-()-\end{array}$ & $\begin{array}{l}2: 8082: 838 \\
2: 786\end{array}$ \\
\hline HA180V & DA & T/C Hardware Alarms & DEV2:N7:228/6 & $4 \mathrm{H} 6$ & $\begin{array}{l}-] /[- \\
-()-\end{array}$ & $\begin{array}{l}2: 8102: 841 \\
2: 787\end{array}$ \\
\hline HA180V & DA & T/C Hardware Alarms & DEV2:N7:228/7 & $4 \mathrm{H} 9$ & $\begin{array}{l}-] /[- \\
-()-\end{array}$ & $\begin{array}{l}2: 811,2: 842 \\
2: 788\end{array}$ \\
\hline HA181B & DA & T/C Hardware Alarms & DEV2:N7:228/8 & 410 & $\begin{array}{l}-] /[- \\
-()-\end{array}$ & $\begin{array}{l}2: 8132: 845 \\
2: 789\end{array}$ \\
\hline HA181B & DA & T/C Hardware Alarms & DEV2:N7:228/9 & 413 & $\begin{array}{l}-] /[- \\
-()-\end{array}$ & $\begin{array}{l}2: 8142: 846 \\
2: 790\end{array}$ \\
\hline HA181V & $\mathrm{DA}$ & T/C Hardware Alarms & DEV2:N7:228/10 & 416 & $\begin{array}{l}-] /[- \\
-()-\end{array}$ & $\begin{array}{l}2: 8162: 849 \\
2: 791\end{array}$ \\
\hline HA181V & DA & T/C Hardware Alarms & DEV2:N7:228/11 & 419 & $\begin{array}{l}-j /[- \\
-()-\end{array}$ & $\begin{array}{l}2: 8172: 850 \\
2: 792\end{array}$ \\
\hline HA182B & DA & T/C Hardware Alarms & DEV2:N7:228/12 & 4J0 & $-] /[-$ & $2: 8192: 853$ \\
\hline
\end{tabular}




\begin{tabular}{|c|c|c|c|c|c|c|}
\hline \multirow{2}{*}{ HA182B } & & & & & -()$-$ & $2: 793$ \\
\hline & DA & T/C Hardware Alarms & DEV2:N7:228/13 & $4 \mathrm{~J} 3$ & $\begin{array}{l}-] / /- \\
-()-\end{array}$ & $\begin{array}{l}2: 8202: 845 \\
2: 794\end{array}$ \\
\hline HA182V & $\mathrm{DA}$ & T/C Hardware Alarms & DEV2:N7:228/14 & $4 J 6$ & $\begin{array}{l}-1 / 4- \\
-()-\end{array}$ & $\begin{array}{l}2: 8222: 857 \\
2: 795\end{array}$ \\
\hline HA182V & DA & T/C Hardware Alarms & DEV2:N7:228/15 & 4J9 & $\begin{array}{l}-1 / 4- \\
-()-\end{array}$ & $\begin{array}{l}2: 8232: 858 \\
2: 796\end{array}$ \\
\hline HA183B & $\mathrm{DA}$ & T/C Hardware Alarms & DEV2:N7:229/0 & $4 \mathrm{KO}$ & $\begin{array}{l}-] /[- \\
-()-\end{array}$ & $\begin{array}{l}2: 8252: 861 \\
2: 797\end{array}$ \\
\hline $\mathrm{HA183B}$ & $\mathrm{DA}$ & T/C Hardware Alarms & DEV2:N7:229/1 & $4 K 3$ & $\begin{array}{l}-] /(- \\
-()-\end{array}$ & $\begin{array}{l}2: 8262: 862 \\
2: 798\end{array}$ \\
\hline HA183V & $\mathrm{DA}$ & T/C Hardware Alarms & DEV2:N7:229/2 & $4 \mathrm{~K} 6$ & $\begin{array}{l}-] /[- \\
-()-\end{array}$ & $\begin{array}{l}2: 8282: 865 \\
2: 799\end{array}$ \\
\hline HA183V & DA & T/C Hardware Alarms & DEV2:N7:229/3 & $4 \mathrm{Kg}$ & $\begin{array}{l}-] /[- \\
-()-\end{array}$ & $\begin{array}{l}2: 8292: 866 \\
2: 800\end{array}$ \\
\hline
\end{tabular}

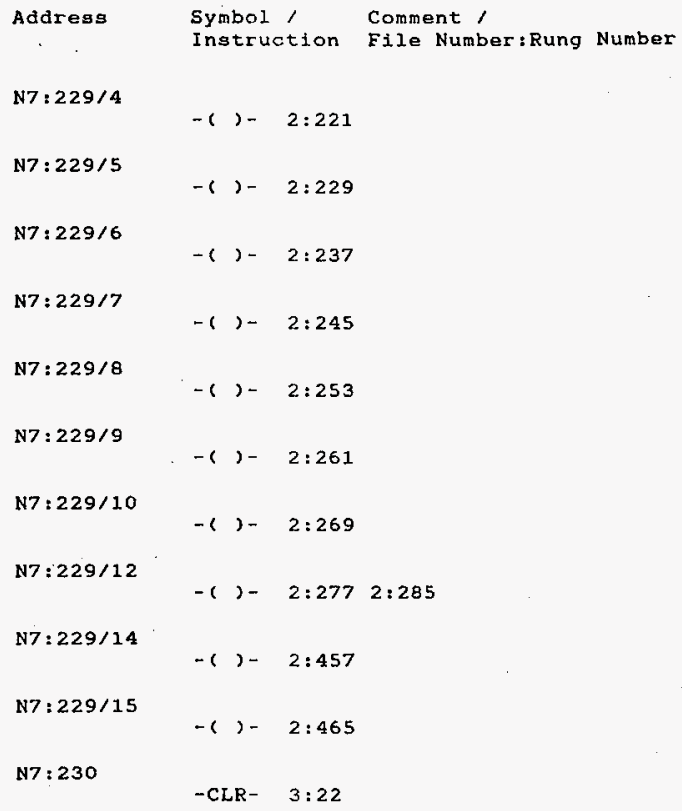




\begin{tabular}{|c|c|c|c|c|c|c|}
\hline & & PID MODE SELECT & DEV2:N7:230 & & CLR & $3: 22$ \\
\hline A_MRD143 & DO & TY-143 AUTO/MAN. & DEV2:N7:230:0 & 4LOO & $-] /[-$ & $2: 218$ \\
\hline ONRD143 & DO & PID SELECT ON/OFF & DEV2:N7:230:1 & & $-] /[-$ & $2: 217$ \\
\hline A_MRD144 & DO & TY-144 AUTO/MAN. & DEV2:N7:230:2 & 4L01 & $-] /[-$ & $2: 226$ \\
\hline ONRD144 & DO & PID SELECT ON/OFF & DEV2:N7:230:3 & & $-1 /[-$ & $2: 225$ \\
\hline A_MRD150 & DO & TY-150 AUTO/MAN. & DEV2:N7:230:4 & 4L02 & $-j / 4-$ & $2: 234$ \\
\hline ONRD150 & DO & PID SELECT ON/OFF & DEV2:N7:230:5 & & $-j /[-$ & $2: 233$ \\
\hline A_MRD151 & DO & TY-151 AUTO/MAN. & DEV2:N7:230:6 & $4 \mathrm{LO} 3$ & $-1 /[-$ & $2: 242$ \\
\hline ONRD151 & DO & PID SELECT ON/OFF & DEV2:N7:230:7 & & $-] /[-$ & $2: 241$ \\
\hline A_MRD152 & DO & TY-152 AUTO/MAN. & DEV2:N7:230:8 & 4L04 & $-] /[-$ & $2: 250$ \\
\hline ONRD152 & DO & PID SELECT ON/OFF & DEV2:N7:230:9 & & $-] /[-$ & $2: 249$ \\
\hline A_MRD153 & DO & TY-153 AUTO/MAN. & DEV2:N7:230:10 & 4L05 & $-] /[-$ & $2: 258$ \\
\hline ONRD153 & DO & PID SELECT ON/OFF & DEV2:N7:230:11 & & $-] /[-$ & $2: 257$ \\
\hline A_MRD154 & DO & TY-154 AUTO/MAN. & DEV2:N7:230:12 & 4LO6 & $-] /[-$ & $2: 266$ \\
\hline ONRD154 & DO & PID SELECT ON/OFF & DEV2:N7:230:13 & & $-] /[-$ & $2: 265$ \\
\hline A_MRD160 & DO & TY-160 AUTO/MAN. & DEV2:N7:230:14 & 4L07 & $-] /[-$ & $2: 274$ \\
\hline \multirow[t]{2}{*}{ ONRD160 } & DO & PID SELECT ON/OFF & DEV2:N7:230:15 & & $-j /[-$ & $2: 373$ \\
\hline & & PID MODE SELECT & DEV2:N7:231 & & CLR & $3: 23$ \\
\hline A_MRD161 & DO & TY-161 AUTO/MAN. & DEV2:N7:231:0 & 4L08 & $-] /[-$ & $2: 282$ \\
\hline ONRD161 & DO & PID SELECT ON/OFF & DEV2:N7:231:1 & & $-] /[-$ & $2: 2812: 501$ \\
\hline A_MRD162 & DO & TY-162 AUTO/MAN. & DEV2:N7:231:2 & 4L09 & $-] /[-$ & $2: 291$ \\
\hline ONRD162 & DO & PID SELECT ON/OFF & DEV2:N7:231:3 & & $-] /[-$ & $2: 290$ \\
\hline A_MRD163 & DO & TY-163 AUTO/MAN. & DEV2:N7:231:4 & $4 L 10$ & $-] /[-$ & $2: 454$ \\
\hline ONRD163 & DO & PID SELECT ON/OFF & DEV2:N7:231:5 & & $-] /[-$ & $2: 453$ \\
\hline \multirow[t]{2}{*}{ A_MRD164 } & DO & TY-164 AUTO/MAN. & DEV2:N7:231:6 & 4L11 & $-3 /[-$ & $2: 462$ \\
\hline & & & & & & $2: 279$ \\
\hline ONRD164 & DO & PID SELECT ON/OFF & DEV2:N7:231:7 & & $-] /[-$ & $2: 461$ \\
\hline A_MRD170B & DO & TY-170B AUTO/MAN. & DEV2:N7:231:8 & 4L12 & $-] /[-$ & $2: 470$ \\
\hline ONRD170B & DO & PID SELECT ON/OFF & DEV2:N7:231:9 & & $-] /[-$ & $2: 469$ \\
\hline A_MRD $170 \mathrm{~V}$ & DO & TY-170V AUTO/MAN. & DEV2:N7:231:10 & $4 L 13$ & $-] /[-$ & $2: 478$ \\
\hline ONRD170V & DO & PID SELECT ON/OFF & DEV2:N7:231:11 & & $-] /[-$ & $2: 477$ \\
\hline A_MRD171B & DO & TY-171B AUTO/MAN. & DEV2:N7:231:12 & 4L14 & $-] /[-$ & $2: 486$ \\
\hline ONRD171B & DO & PID SELECT ON/OFF & DEV2:N7:231:13 & & $-] /[-$ & $2: 485$ \\
\hline A_MRD171V & DO & TY-171V AUTO/MAN. & DEV2:N7:231:14 & $4 L 15$ & $-] /[-$ & $2: 494$ \\
\hline \multirow[t]{2}{*}{ ONRD171V } & DO & PID SELECT ON/OFF & DEV2:N7:231:15 & & $-] /[-$ & $2: 493$ \\
\hline & & PID MODE SELECT & DEV2:N7:232 & & CLR & $3: 24$ \\
\hline A_MRD172B & DO & TY-172B AUTO/MAN. & DEV2:N7:232:0 & $4 L 16$ & $-] /[-$ & $2: 502$ \\
\hline ONRD172B & DO & PID SELECT ON/OFF & DEV2:N7:232:1 & & $-] /[-$ & \\
\hline A_MRD172V & DO & TY-172V AUTO/MAN. & DEV2:N7:232:2 & 4L17 & $-] /[-$ & $2: 510$ \\
\hline ONRD172V & DO & PID SELECT ON/OFF & DEV2:N7:232:3 & & & $2: 509$ \\
\hline A_MRD173B & DO & TY-173B AUTO/MAN. & DEV2:N7:232:4 & 4L18 & $-] /[-$ & $2: 518$ \\
\hline ONRD173B & DO & PID SELECT ON/OFF & DEV2:N7:232:5 & & $-] /[-$ & $2: 517$ \\
\hline A_MRD173V & DO & TY-173V AUTO/MAN. & DEV2:N7:232:6 & 4L19 & $-] /[-$ & $2: 526$ \\
\hline ONRD173V & DO & PID SELECT ON/OFF & DEV2:N7:232:7 & & $-] /[-$ & $2: 525$ \\
\hline A_MRD174B & DO & TY-174B AUTO/MAN. & DEV2:N7:232:8 & $4 L 20$ & $-] /[-$ & $2: 534$ \\
\hline ONRD174B & DO & PID SELECT ON/OFF & DEV2:N7:232:9 & & $-] /[-$ & $2: 533$ \\
\hline A_MRD174V & DO & TY-174V AUTO/MAN. & DEV2:N7:232:10 & $4 L 21$ & $-] /[-$ & $2: 543$ \\
\hline ONRD174V & DO & PID SELECT ON/OFF & DEV2:N7:232:11 & & $-] /[-$ & $2: 542$ \\
\hline A MRD175B & DO & TY-175B AUTO/MAN. & DEV2:N7:232:12 & 4L22 & $-] /[-$ & $2: 676$ \\
\hline ONRD175B & DO & PID SELECT ON/OFF & DEV2:N7:232:13 & & $-3 /[-$ & $2: 675$ \\
\hline A_MRD $175 \mathrm{~V}$ & DO & TY-175V AUTO/MAN. & DEV2:N7:232:14 & $4\llcorner 23$ & $-] /[-$ & $2: 684$ \\
\hline ONRD175V & DO & PID SELECT ON/OFF & DEV2:N7:232:15 & & $-] /[-$ & $2: 683$ \\
\hline
\end{tabular}




\begin{tabular}{|c|c|c|c|c|c|c|}
\hline & & PID MODE SELECT & DEV2:N7:233 & & CLR & $3: 25$ \\
\hline A_MRD176B & DO & TY-176B AUTO/MAN. & DEV2:N7:233:0 & $4 \mathrm{~L} 24$ & $-] /[-$ & $2: 692$ \\
\hline ONRD176B & DO & PID SELECT ON/OFF & DEV2:N7:233:1 & & & $2: 691$ \\
\hline A_MRD176V & DO & TY-176V AUTO/MAN. & DEV2:N7:233:2 & $4 \mathrm{~L} 25$ & & $2: 700$ \\
\hline ONRD176V & DO & PID SELECT ON/OFF & DEV2:N7:233:3 & & $-j /[-$ & $2: 699$ \\
\hline A_MRD177B & $\mathrm{DO}$ & TY-177B AUTO/MAN. & DEV2:N7:233:4 & $4 \mathrm{~L} 26$ & $-] /[-$ & $2: 708$ \\
\hline ONRD177B & DO & PID SELECT ON/OFF & DEV2:N7:233:5 & & $-j /[-$ & $2: 7072: 710$ \\
\hline A_MRD177V & DO & TY-177V AUTO/MAN. & DEV2:N7:233:6 & $4 \mathrm{~L} 27$ & $-1] /[-$ & $2: 716$ \\
\hline ONRD177V & DO & PID SELECT ON/OFF & DEV2:N7:233:7 & & $-] /[-$ & $2: 7152: 718$ \\
\hline A_MRD178B & DO & TY-178B AUTO/MAN. & DEV2:N7:233:8 & $4 \mathrm{~L} 28$ & $-] /[-$ & $2: 724$ \\
\hline ONRD178B & DO & PID SELECT ON/OFF & DEV2:N7:233:9 & & $-] /[-$ & $2: 723$ \\
\hline A MRD $178 \mathrm{~V}$ & $\mathrm{DO}$ & TY-178V AUTO/MAN. & DEV2:N7:233:10 & $4 \mathrm{~L} 29$ & $-1 /[-$ & $2: 732$ \\
\hline ONRD178V & DO & PID SELECT ON/OFF & DEV2:N7:233:11 & & $-1 /[-$ & $2: 731$ \\
\hline A_MRD179B & DO & TY-179B AUTO/MAN. & DEV2:N7:233:12 & $4 \mathrm{~L} 30$ & $-] /[-$ & $2: 741$ \\
\hline ONRD179B & DO & PID SELECT ON/OFF & DEV2:N7:233:13 & & $-] /[-$ & $2: 740$ \\
\hline A_MRD179V & $\mathrm{DO}$ & TY-179B AUTO/MAN. & DEV2:N7:233:14 & 4L31 & & $2: 874$ \\
\hline \multirow[t]{2}{*}{ ONRD179V } & DO & PID SELECT ON/OFF & DEV2:N7:233:15 & & & $2: 873$ \\
\hline & & PID MODE SELECT & DEV2:N7:234 & & & $3: 26$ \\
\hline A_MRD180B & DO & TY-180B AUTO/MAN. & DEV2:N7:234:0 & $4 \mathrm{M} 00$ & $-1 /[-$ & $2: 882$ \\
\hline ONRD180B & DO & PID SELECT ON/OFF & DEV2:N7:234:1 & & $-] /[-$ & $2: 881$ \\
\hline A_MRD180V & DO & TY-180V AUTO/MAN. & DEV2:N7:234:2 & 4M01 & $-] /[-$ & $2: 890$ \\
\hline ONRD180V & DO & PID SELECT ON/OFF & DEV2:N7:234:3 & & $-][-$ & $2: 889$ \\
\hline A_MRD181B & DO & TY-181B AUTO/MAN. & DEV2:N7:234:4 & 4M02 & $-] /[-$ & $2: 898$ \\
\hline ONRD181B & DO & PID SELECT ON/OFF & DEV2:N7:234:5 & & $-j /[-$ & $2: 897$ \\
\hline A_MRD181V & DO & TY-181V AUTO/MAN. & DEV2:N7:234:6 & $4 \mathrm{M} 03$ & $-] /[-$ & $2: 906$ \\
\hline ONRD181V & DO & PID SELECT ON/OFF & DEV2:N7:234:7 & & $-] /[-$ & $2: 905$ \\
\hline A_MRD182B & DO & TY-182B AUTO/MAN. & DEV2:N7:234:8 & $4 \mathrm{M} 04$ & $-] /[-$ & $2: 914$ \\
\hline ONRD182B & DO & PID SELECT ON/OFF & DEV2:N7:234:9 & & $-] /[-$ & $2: 913$ \\
\hline A MRD182V & DO & TY-182V AUTO/MAN. & DEV2:N7:234:10 & $4 M 05$ & $-] /[-$ & $2: 922$ \\
\hline ONRD182V & DO & PID SELECT ON/OFF & DEV2:N7:234:11 & & $-j /[-$ & $2: 921$ \\
\hline A MRD183B & DO & TY-183B AUTO/MAN. & DEV2:N7:234:12 & 4MO6 & $-j /[-$ & $2: 930$ \\
\hline ONRD183B & DO & PID SELECT ON/OFF & DEV2:N7:234:13 & & $-] /[-$ & $2: 929$ \\
\hline A_MRD $183 \mathrm{~V}$ & DO & TY-183V AUTO/MAN. & DEV2:N7:234:14 & $4 \mathrm{M} 07$ & $-] /[-$ & $2: 939$ \\
\hline
\end{tabular}


Processor and DataloPS Unit 1 )

Address

Symbol / Instruction

Comment 1

File Number:Rung Number

N7 $: 235 / 0$

$$
-()-2: 473
$$

N7 : $235 / 1$

$$
-(3-2: 481
$$

N7 $: 235 / 2$

$$
-()-2: 489
$$

N7 $: 235 / 3$

$$
-()-2: 497
$$

N7 : $235 / 4$

$$
-()-2: 505
$$

N7:235/5

$$
-()-2: 513
$$

N7 : $235 / 6$

\section{SCRATCH}

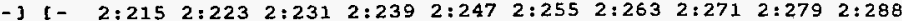
$\begin{array}{llllllllll}2: 451 & 2: 459 & 2: 467 & 2: 475 & 2: 483 & 2: 499 & 2: 507 & 2: 515 & 2: 523 & 2: 531\end{array}$ $\begin{array}{llllllllll}2: 540 & 2: 673 & 2: 681 & 2: 689 & 2: 697 & 2: 705 & 2: 713 & 2: 721 & 2: 729 & 2: 737\end{array}$

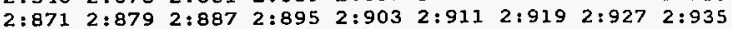

$-] /[-2: 491$

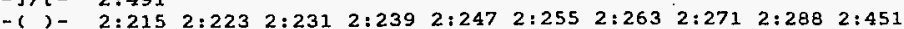
$\begin{array}{llllllllll}2: 459 & 2: 467 & 2: 475 & 2: 483 & 2: 491 & 2: 499 & 2: 507 & 2: 515 & 2: 523 & 2: 531\end{array}$ $\begin{array}{llllllllll}2: 540 & 2: 673 & 2: 681 & 2: 689 & 2: 697 & 2: 705 & 2: 713 & 2: 721 & 2: 729 & 2: 737\end{array}$

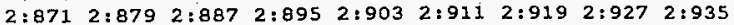

$N 7: 235 / 7$

-()$-2: 521$

N7 $: 235 / 8$

$-6)-2: 529$

N7 $: 235 / 9$

$$
-()-2: 537
$$

HNF-SD-FF-CSWD-60Rev. 0 
Processor and Data(ops Unit 1 )

\section{Cross Reference}

Processor File: SODIUM2A.ACH

October 23, 1996

Address

Symbol

Comment /

Instruction File Number:Rung Number

N7: $235 / 11$

-()$-2: 679$

N7 : 235/12

$-(1)-2: 687$

N7 : $235 / 13$

$-(2)-2: 695$

N7 : 235/14

-()$-2: 703$

N7 : $235 / 15$

-()$-2: 711$

N7 $: 236 / 0$

-()$-2: 719$

N7 : 236/1

$-(2)-2: 727$

N7 : $236 / 2$

$-(1)-2: 735$

N7 : $236 / 4$

-()$-2: 877$

N7 : $236 / 5$

$-(2)-2: 885$

N7 $: 236 / 6$

-()$-2: 893$

N7 : $236 / 7$

-()$-2: 9012: 909$

N7 : $236 / 9$

-()$-2: 917$

$N 7: 236 / 10$

-()$-2: 925$

$N 7: 236 / 11$

-()$-2: 933$

HNF-SD-FF-CSWD-60Rev. 0 


\begin{tabular}{|c|c|c|c|c|c|}
\hline OUT143A & DO & TE TAKE-OUT & DEV2:N7:236:12 & $-] /[-$ & $2: 170$ \\
\hline OUT143B & DO & TE TAKE-OUT & DEV2:N7:236:13 & $-] /[-$ & $2: 171$ \\
\hline OUT144A & DO & TE TAKE-OUT & DEV2:N7:236:14 & $-] /[-$ & $2: 174$ \\
\hline OUT144B & DO & TE TAKE-OUT & DEV2:N7:236:15 & $-] /[-$ & $2: 175$ \\
\hline OUT150A & DO & TE TAKE-OUT & DEV2:N7:237:0 & $-] /[-$ & $2: 178$ \\
\hline OUT150B & DO & TE TAKE-OUT & DEV2:N7:237:1 & $-] /[-$ & $2: 179$ \\
\hline OUT150C & DO & TE TAKE-OUT & DEV2:N7:237:2 & $-] /[-$ & $2: 180$ \\
\hline OUT151A & DO & TE TAKE-OUT & DEV2:N7:237:3 & $-] /[-$ & $2: 183$ \\
\hline OUT151B & DO & TE TAKE-OUT & DEV2:N7:237:4 & $-] /[-$ & $2: 184$ \\
\hline OUT151C & DO & TE TAKE-OUT & DEV2:N7:237:5 & $-] /[-$ & $2: 185$ \\
\hline OUT152A & DO & TE TAKE-OUT & DEV2:N7:237:6 & $-] /[-$ & $2: 188$ \\
\hline OUT152B & DO & TE TAKE-OUT & DEV2:N7:237:7 & $-j / 1-$ & $2: 189$ \\
\hline OUT153A & DO & TE TAKE-OUT & DEV2:N7:237:8 & $-] /[-$ & $2: 192$ \\
\hline OUT153B & DO & TE TAKE-OUT & DEV2:N7:237:9 & $-] /[-$ & $2: 193$ \\
\hline OUT154A & DO & TE TAKE-OUT & DEV2:N7:237:10 & $-] /[-$ & $2: 196$ \\
\hline OUT154B & DO & TE TAKE-OUT & DEV2:N7:237:11 & $-j /[-$ & $2: 197$ \\
\hline OUT160A & DO & TE TAKE-OUT & DEV2:N7:237:12 & $-] /[-$ & $2: 200$ \\
\hline OUT160B & DO & TE TAKE-OUT & DEV2:N7:237:13 & $-] /[-$ & 2:201 \\
\hline OUT160C & DO & TE TAKE-OUT & DEV2:N7:237:14 & $-j /[-$ & $2: 202$ \\
\hline OUT161A & DO & TE TAKE-OUT & DEV2:N7:237:15 & $-j /[-$ & $2: 205$ \\
\hline OUT161B & DO & TE TAKE-OUT & DEV2:N7:238:0 & $-j /[-$ & $2: 206$ \\
\hline OUT161C & DO & TE TAKE-OUT & DEV2:N7:238:1 & $-] /[-$ & $2: 207$ \\
\hline OUT162A & DO & TE TAKE-OUT & DEV2:N7:238:2 & $-] /[-$ & $2: 210$ \\
\hline OUT162B & DO & TE TAKE-OUT & DEV2:N7:238:3 & $\cdot] /[-$ & $2: 211$ \\
\hline OUT163A & DO & TE TAKE-OUT & DEV2:N7:238:4 & $-j / 2$ & $2: 402$ \\
\hline OUT163B & DO & TE TAKE-OUT & DEV2:N7:238:5 & $-] /[-$ & $2: 403$ \\
\hline OUT.164A & DO & TE TAKE-OUT & DEV2:N7:238:6 & $-] /[-$ & $2: 406$ \\
\hline OUT164B & DO & TE TAKE-OUT & DEV2:N7:238:7 & $-] /[-$ & $2: 407$ \\
\hline OUT170B1 & DO & TE TAKE-OUT & DEV2:N7:238:8 & $-] /[-$ & $2: 410$ \\
\hline OUT170B2 & DO & TE TAKE-OUT & DEV2:N7:238:9 & $-] /[-$ & $2: 411$ \\
\hline OUT170V1 & DO & TE TAKE-OUT & DEV2:N7:238:10 & $-] /[-$ & $2: 414$ \\
\hline OUT170V2 & DO & TE TAKE-OUT & DEV2:N7:238:11 & $-] /[-$ & $2: 415$ \\
\hline OUT171B1 & DO & TE TAKE-OUT & DEV2:N7:238:12 & $-] /[-$ & $2: 418$ \\
\hline OUT171B2 & DO & TE TAKE-OUT & DEV2:N7:238:13 & $-] /[-$ & $2: 419$ \\
\hline OUT171V1 & DO & TE TAKE-OUT & DEV2:N7:238:14 & $-] /[-$ & $2: 422$ \\
\hline OUT171V2 & DO & TE TAKE-OUT & DEV2:N7:239:15 & $-] /[-$ & $2: 423$ \\
\hline OUT172B1 & DO & TE TAKE-OUT & DEV2:N7:239:0 & $-1 /[-$ & $2: 426$ \\
\hline OUT172B2 & DO & TE TAKE-OUT & DEV2:N7:239:1 & $-] /[-$ & $2: 427$ \\
\hline OUT172V1 & DO & TE TAKE-OUT & DEV2:N7:239:2 & $-] /[-$ & $2: 430$ \\
\hline OUT172V2 & DO & TE TAKE-OUT & DEV2:N7:239:3 & $-] /[-$ & $2: 431$ \\
\hline OUT173B1 & DO & TE TAKE-OUT & DEV2:N7:239:4 & $-] /[-$ & $2: 434$ \\
\hline OUT173B2 & DO & TE TAKE-OUT & DEV2:N7:239:5 & $-] /[-$ & $2: 435$ \\
\hline OUT173V1 & DO & TE TAKE-OUT & DEV2:N7:239:6 & $-] /[-$ & $2: 438$ \\
\hline OUT173V2 & DO & TE TAKE-OUT & DEV2:N7:239:7 & $-] /[-$ & $2: 439$ \\
\hline OUT174B1 & DO & TE TAKE-OUT & DEV2:N7:239:8 & $-] /[-$ & $2: 442$ \\
\hline OUT174B2 & DO & TE TAKE-OUT & DEV2:N7:239:9 & $-] /[-$ & $2: 443$ \\
\hline OUT174V1 & DO & TE TAKE-OUT & DEV2:N7:239:10 & $-] /[-$ & $2: 446$ \\
\hline OUT174V2 & DO & TE TAKE-OUT & DEV2:N7:239:11 & $-] /[-$ & $2: 447$ \\
\hline OUT175B1 & DO & TE TAKE-OUT & DEV2:N7:239:12 & $-] /[-$ & $2: 635$ \\
\hline OUT175B2 & DO & TE TAKE-OUT & DEV2:N7:239:13 & $-] /[-$ & $2: 636$ \\
\hline OUT175V1 & DO & TE TAKE-OUT & DEV2:N7:239:14 & $-] /[-$ & $2: 639$ \\
\hline
\end{tabular}




\begin{tabular}{|c|c|c|c|c|c|}
\hline OUT175V2 & DO & TE TAKE-OUT & DEV2:N7:239:15 & $-] /[-$ & $2: 640$ \\
\hline OUT176B1 & DO & TE TAKE-OUT & DEV2:N7:240:0 & $-] /[-$ & $2: 643$ \\
\hline OUT176B2 & DO & TE TAKE-OUT & DEV2:N7:240:1 & $-] /[-$ & $2: 644$ \\
\hline OUT176V1 & DO & TE TAKE-OUT & DEV2:N7:240:2 & $-] /[-$ & $2: 647$ \\
\hline OUT176V2 & DO & TE TAKE-OUT & DEV2:N7:240:3 & $-] /[-$ & $2: 648$ \\
\hline OUT177B1 & DO & TE TAKE-OUT & DEV2:N7:240:4 & $-] /[-$ & $2: 651$ \\
\hline OUT177B2 & DO & TE TAKE-OUT & DEV2:N7:240:5 & $-] /[-$ & $2: 652$ \\
\hline OUT177V1 & DO & TE TAKE-OUT & DEV2:N7:240:6 & $-]_{/-}^{-}$ & $2: 655$ \\
\hline OUT177V2 & DO & TE TAKE-OUT & DEV2:N7:240:7 & $-] /[-$ & $2: 656$ \\
\hline OUT178B1 & DO & TE TAKE-OUT & DEV2:N7:240:8 & $-1 /[-$ & $2: 659$ \\
\hline OUT178B2 & DO & TE TAKE-OUT & DEV2:N7:240:9 & $-] /[-$ & $2: 660$ \\
\hline OUT178V1 & DO & TE TAKE-OUT & DEV2:N7:240:10 & $-] /[-$ & $2: 663$ \\
\hline OUT178V2 & DO & TE TAKE-OUT & DEV2:N7:240:11 & $-] /[-$ & $2: 664$ \\
\hline OUT179B1 & DO & TE TAKE-OUT & DEV2:N7:240:12 & $-] /[-$ & $2: 667$ \\
\hline OUT179B2 & DO & TE TAKE-OUT & DEV2:N7:240:13 & $-] /[-$ & $2: 668$ \\
\hline OUT179V1 & DO & TE TAKE-OUT & DEV2:N7:240:14 & $-] /[-$ & $2: 833$ \\
\hline OUT179V2 & DO & TE TAKE-OUT & DEV2:N7:240:15 & $-] /[-$ & $2: 834$ \\
\hline OUT180B1 & DO & TE TAKE-OUT & DEV2:N7:241:0 & $-] /[-$ & $2: 837$ \\
\hline OUT180B2 & DO & TE TAKE-OUT & DEV2:N7:241:1 & $-] /[-$ & $2: 838$ \\
\hline OUT180V1 & DO & TE TAKE-OUT & DEV2:N7:241:2 & $-] /[-$ & $2: 841$ \\
\hline OUT180V2 & DO & TE TAKE-OUT & DEV2:N7:241:3 & $-] /[-$ & $2: 842$ \\
\hline OUT181B1 & DO & TE TAKE-OUT & DEV2:N7:241:4 & $-] / 1-$ & $2: 845$ \\
\hline OUT18182 & DO & TE TAKE-OUT & DEV2:N7:241:5 & $-] /[-$ & $2: 846$ \\
\hline OUT181V1 & DO & TE TAKE-OUT & DEV2:N7:241:6 & $-] /[-$ & $2: 849$ \\
\hline OUT181V2 & DO & TE TAKE-OUT & DEV2:N7:241:7 & $-] /[-$ & $2: 850$ \\
\hline OUT182B1 & DO & TE TAKE-OUT & DEV2:N7:241:8 & $-] /[-$ & $2: 853$ \\
\hline OUT182B2 & DO & TE TAKE-OUT & DEV2:N7:241:9 & $-] /[-$ & $2: 854$ \\
\hline OUT182V1 & DO & TE TAKE-OUT & DEV2:N7:241:10 & $-] /[-$ & $2: 857$ \\
\hline OUT182V2 & DO & TE TAKE-OUT & DEV2:N7:241:11 & $-] /[-$ & $2: 858$ \\
\hline OUT183B1 & DO & TE TAKE-OUT & DEV2:N7:241:12 & $-] /[-$ & $2: 861$ \\
\hline OUT183B2 & DO & TE TAKE-OUT & DEV2:N7:241:13 & $-] /[-$ & $2: 862$ \\
\hline OUT183V1 & DO & TE TAKE-OUT & DEV2:N7:241:14 & $-] /[-$ & $2: 865$ \\
\hline OUT183V2 & DO & TE TAKE-OUT & DEV2:N7:241:15 & $-] /[-$ & $2: 866$ \\
\hline
\end{tabular}


Processor and Data(ops Unit 1)

Cross Reference

Processor File: SODIUM2A.ACH

N10:1

PW 143

- CLR- 2:217

-LES- $2: 945$

-SCL- $2: 220$

$\mathrm{N} 10: 2$

PW144

-CLR- $2: 225$

- LES- $2: 948$

$-S C L-2: 228$

N10:3

PW150

-CLR- 2:233

-LES- $2: 951$

-SCL- 2:236

N10: 4

PW151

-CLR- 2:241

-LES- 2:954

-SCL- 2:244

N10: 5

PWI 52

-CLR- 2:249

-LES- 2:957

$-S C L-\quad 2: 252$

N10: 6

PW153

-CLR- 2:257

-LES - 2:960

-SCL - 2:260

$\begin{array}{lll}\text { N10:7 } & \text { PW154 } \\ & \text {-CLR- } & 2: 265 \\ & \text {-LES- } & 2: 963 \\ & \text {-SCL- } & 2: 268\end{array}$

N10:8

PW 160

-CLR - $2: 273$

-LES- 2:966

-SCL- 2:276

N10: 9

PW161

-CLR- 2:281

-LES- 2:969

- SCL- $2: 284$

HNF-SD-FF-CSWD-60 Rev. 0 


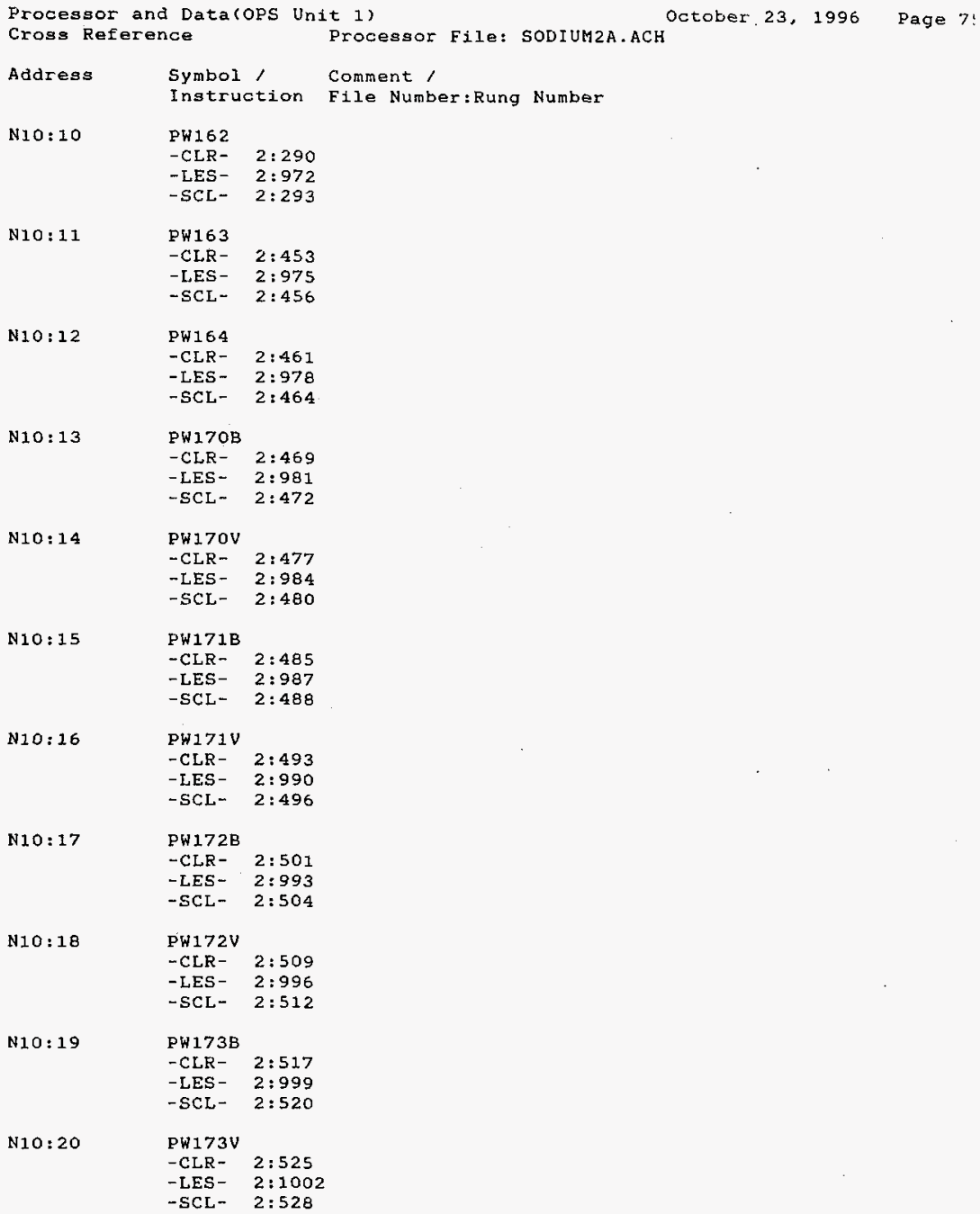

N10: 19

PW173B

-CLR- 2:517

-LES- 2:999

$-\mathrm{SCL}-2: 520$

N10:20

PW173V

-CLR- 2:525

-LES- 2:1002

-SCL- 2:528

HNF-SD-FF-CSWD-6DRev. 0 


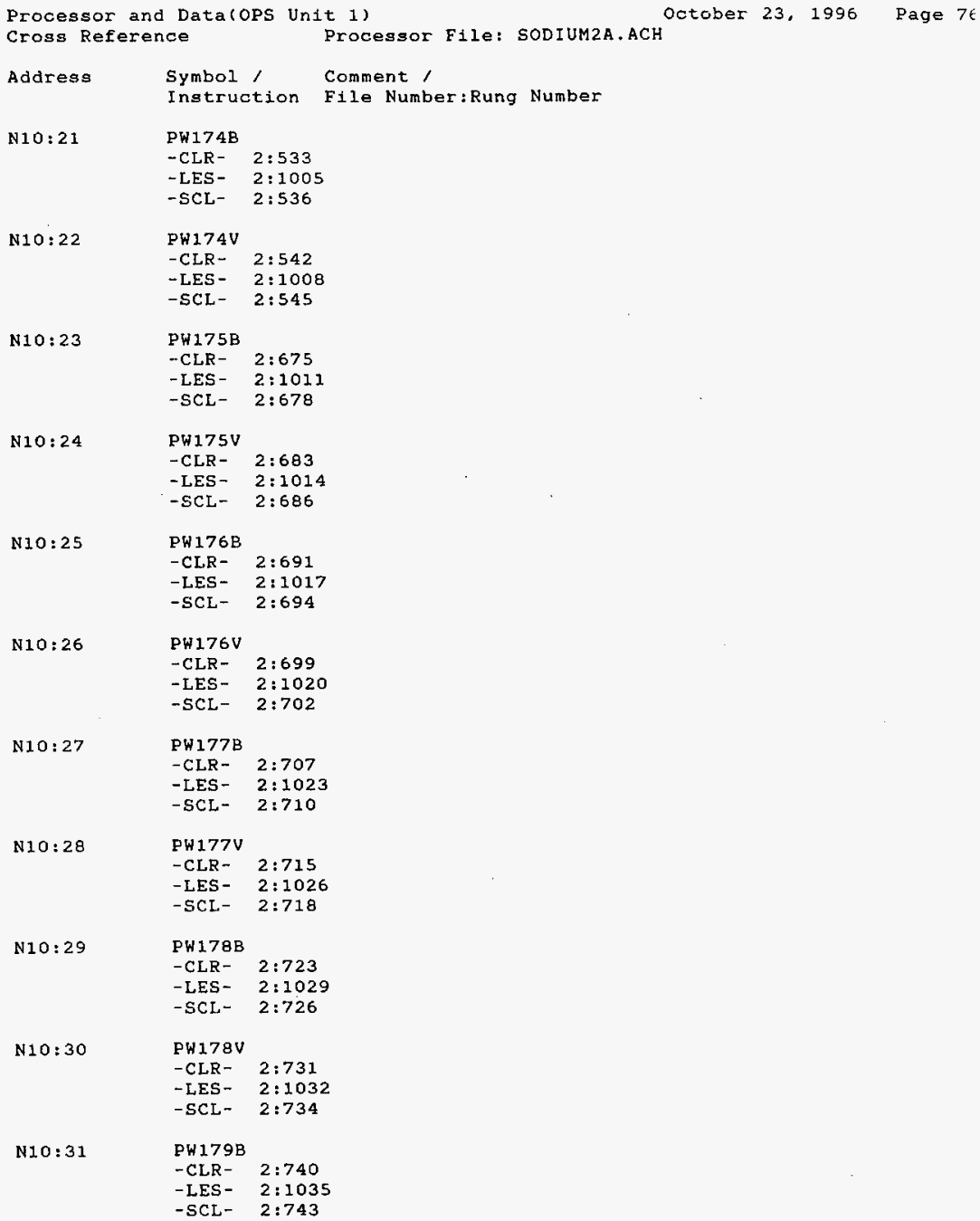

N10: 27

PWI77B

- CLR- 2:707

-LES- 2:1023

-SCL - 2:710

N10: 28

PW177V

-CLR - 2:715

-LES- 2:1026

-SCL- 2:718

N10: 29

PW178B

-CLR- 2:723

-LES- 2:1029

SCL- $2: 726$

$N 10: 30$

PW178V

-CLR- 2:731

-LES - 2:1032

SCL- 2:734

N10:31

PW179B

-CLR- 2:740

-LES- 2:1035

-SCL- $2: 743$

HNF-SD-FF-CSWD-6DRev, 0

Page 163 


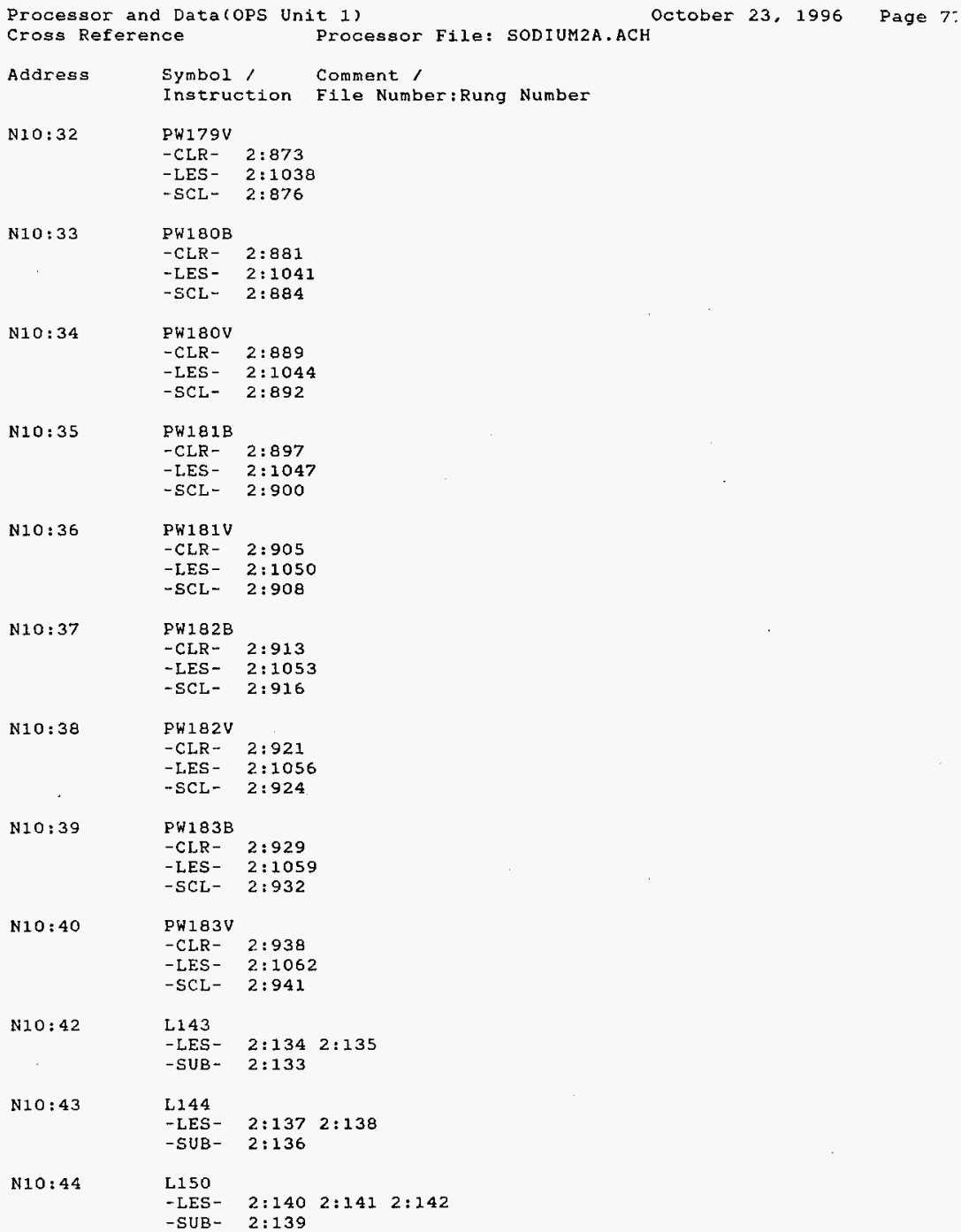

$\begin{array}{lll}\text { N10:40 } & \text { PW183V } \\ & \text {-CLR- } 2: 938 \\ & \text {-LES- } 2: 1062 \\ & \text {-SCL- } 2: 941 \\ \text { N10:42 } & & \\ & \text { L143 } & \\ & \text {-LES- } 2: 134 \quad 2: 135 \\ & \text {-SUB- } 2: 133 \\ \text { N10:43 } & & \\ & \text { L144 } & \\ & \text {-LES- } 2: 137 \quad 2: 138 \\ \text { N10:44 } & \text {-SUB- } 2: 136 \\ & \text { L150 } \\ & \text {-LES- } 2: 140 \quad 2: 141 \quad 2: 142 \\ & \text {-SUB- } 2: 139\end{array}$

HNF-SD-FF-CSWD-60Rev. 0 


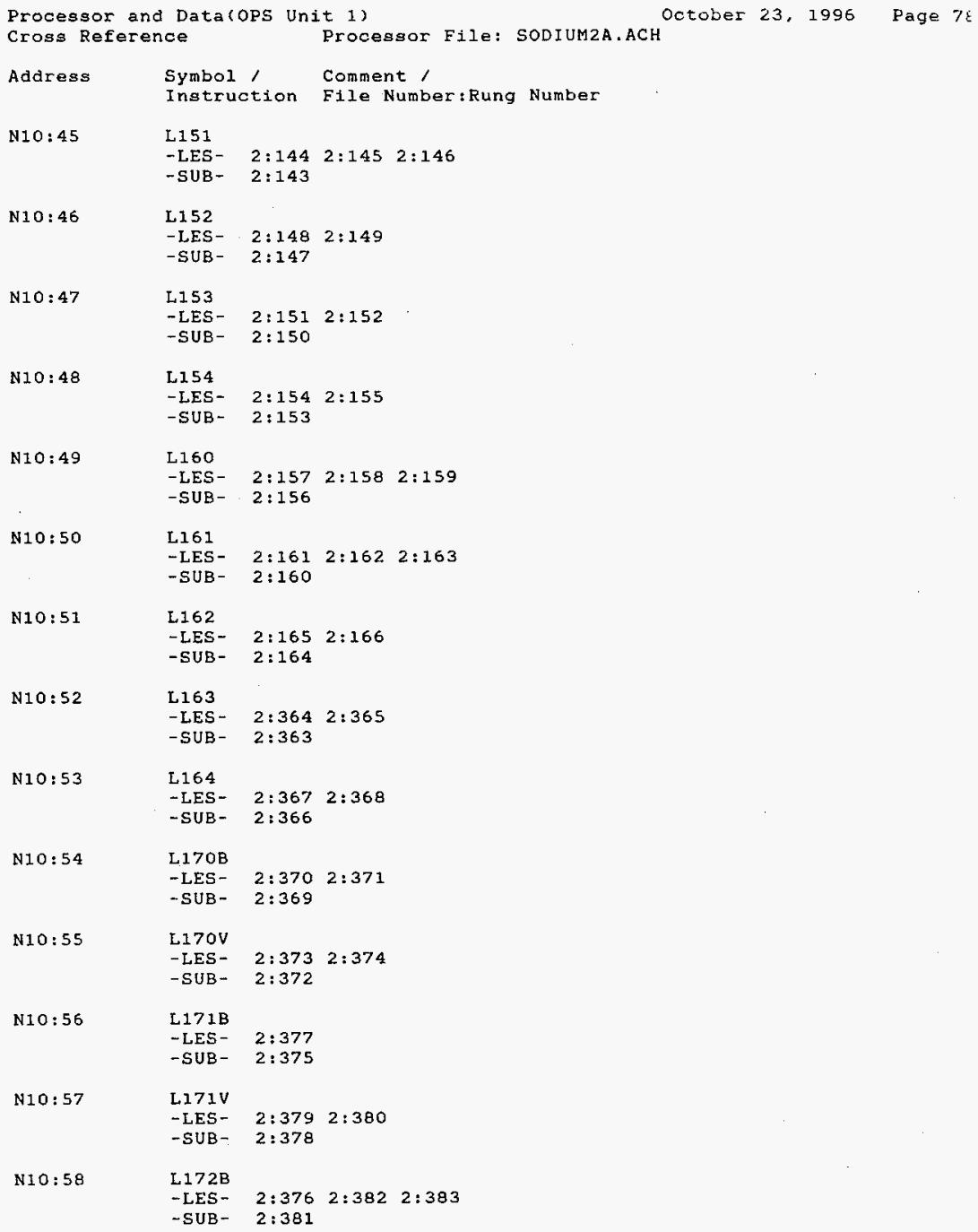




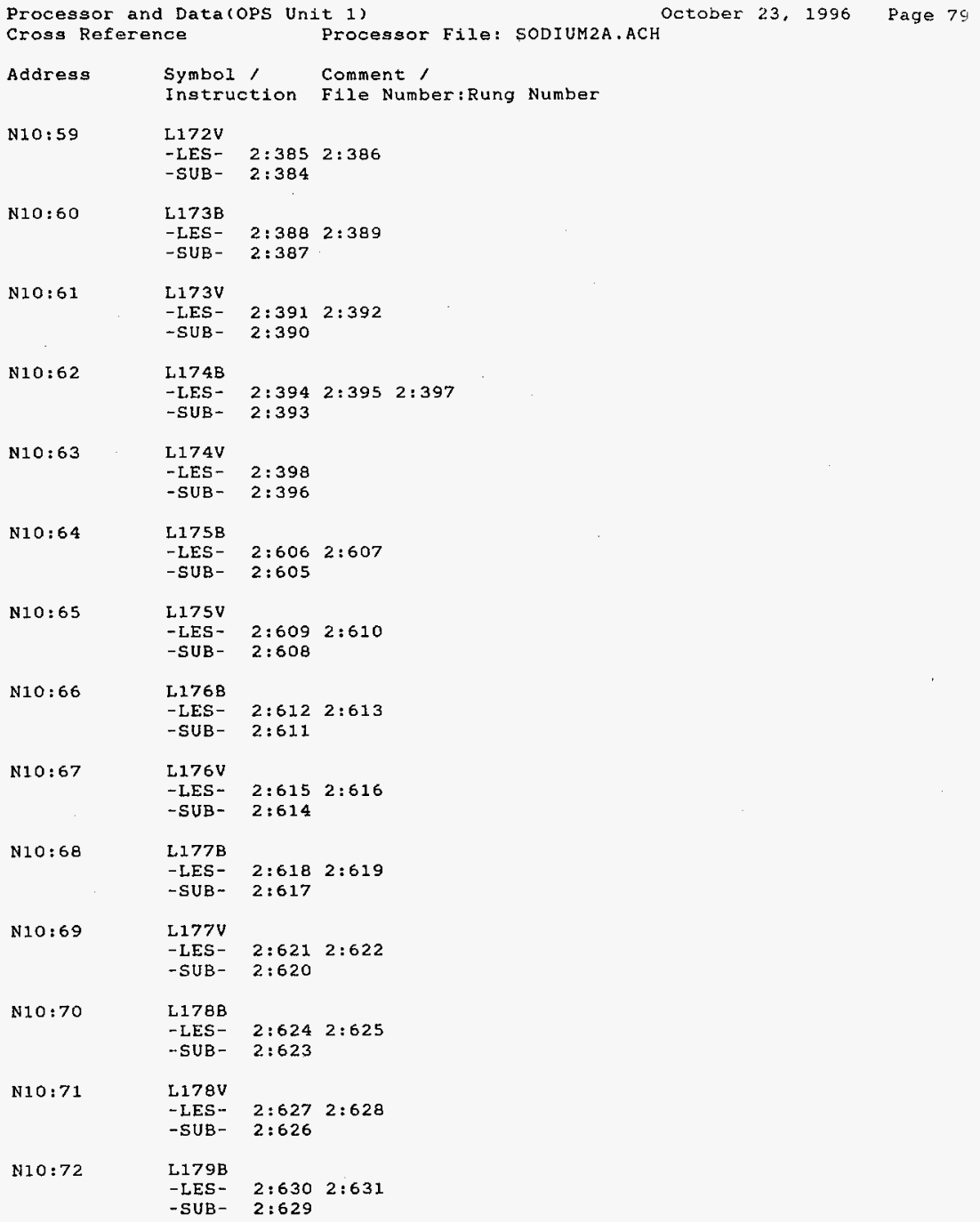

HNF-SD-FF-CSWD-60Rev. 0 


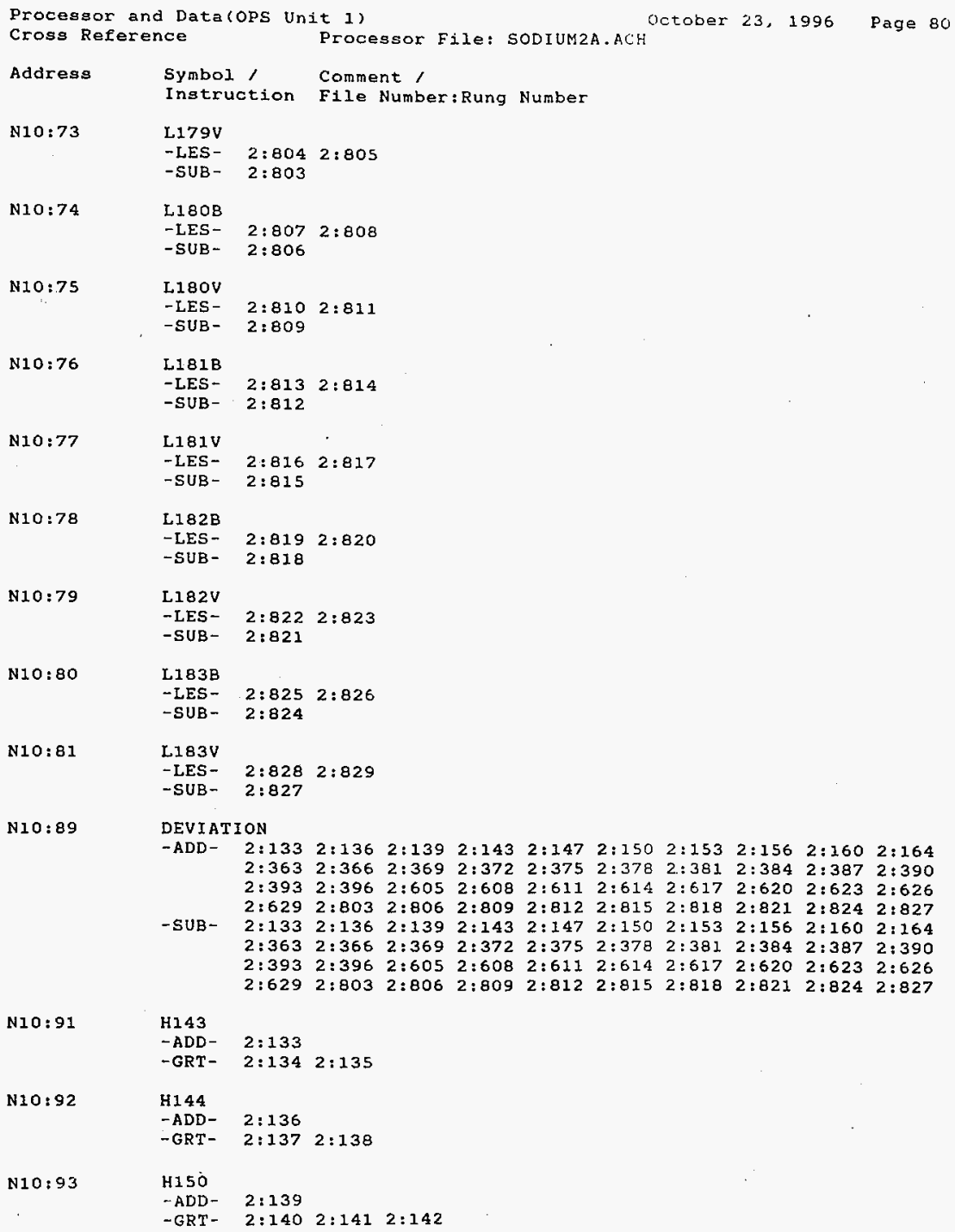

HNF-SD-FF-CSWD-60Rev. 0 


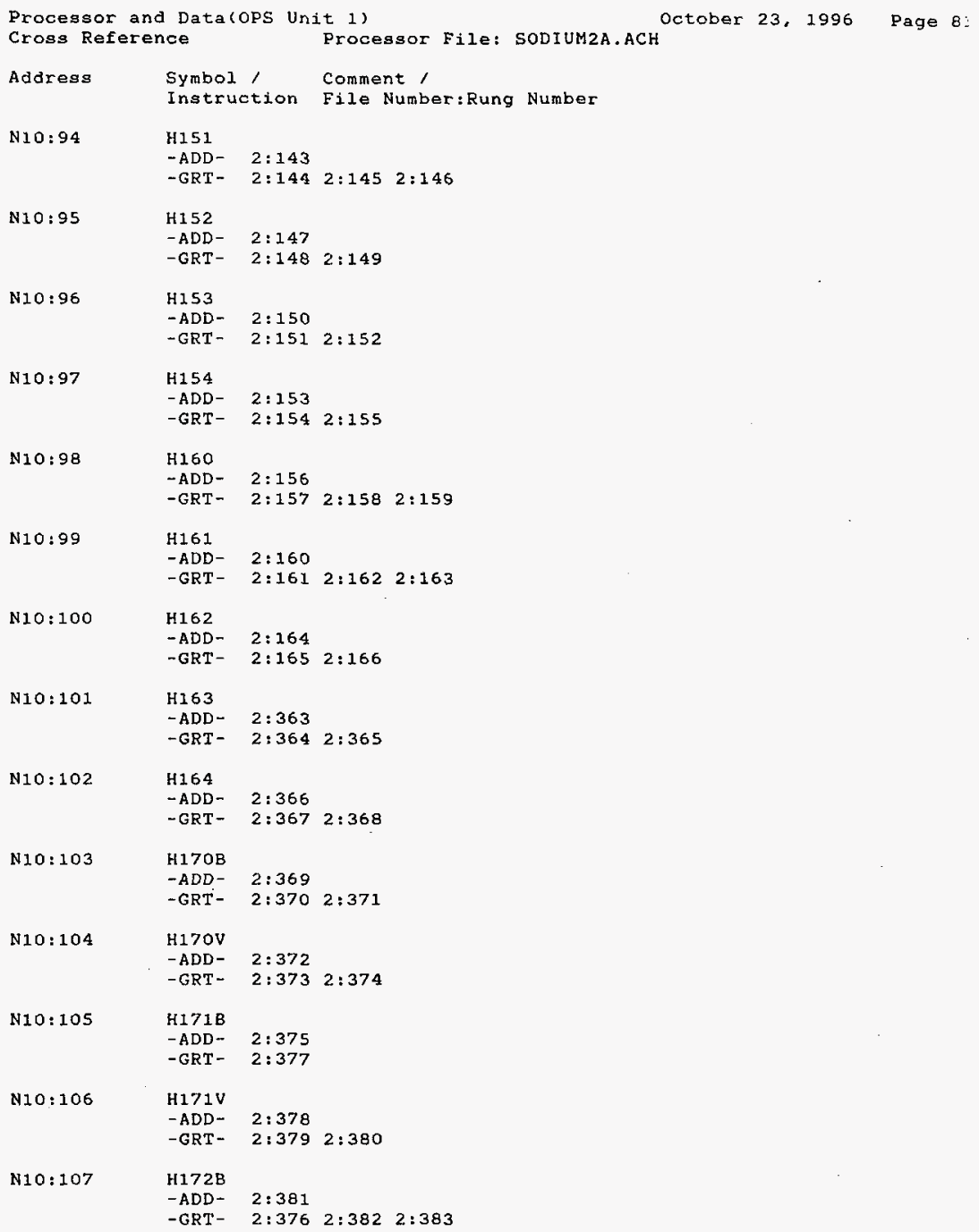

HNF-SD-FF-CSWD-60Rev. 0 


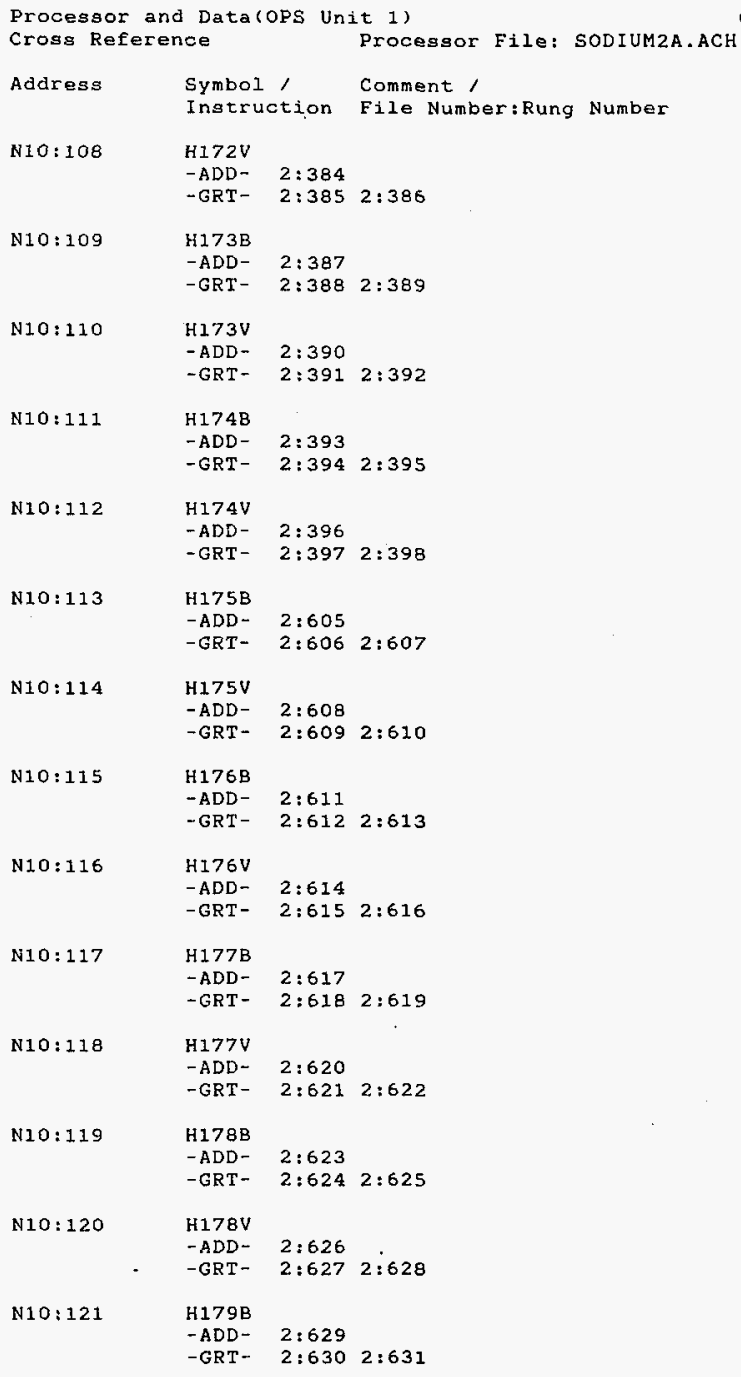

HNF-SD-FF-CSWD-60 Rev. 0 


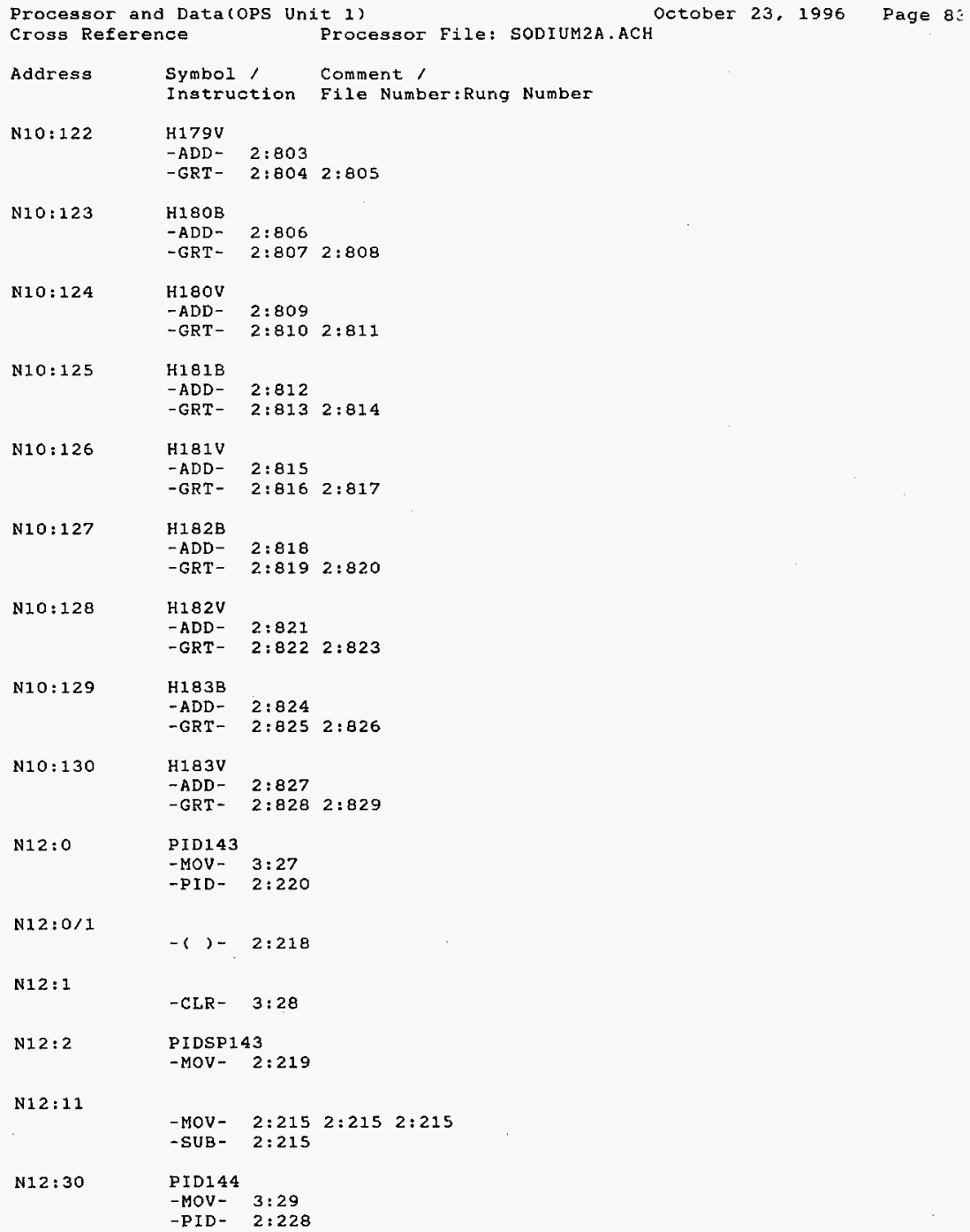




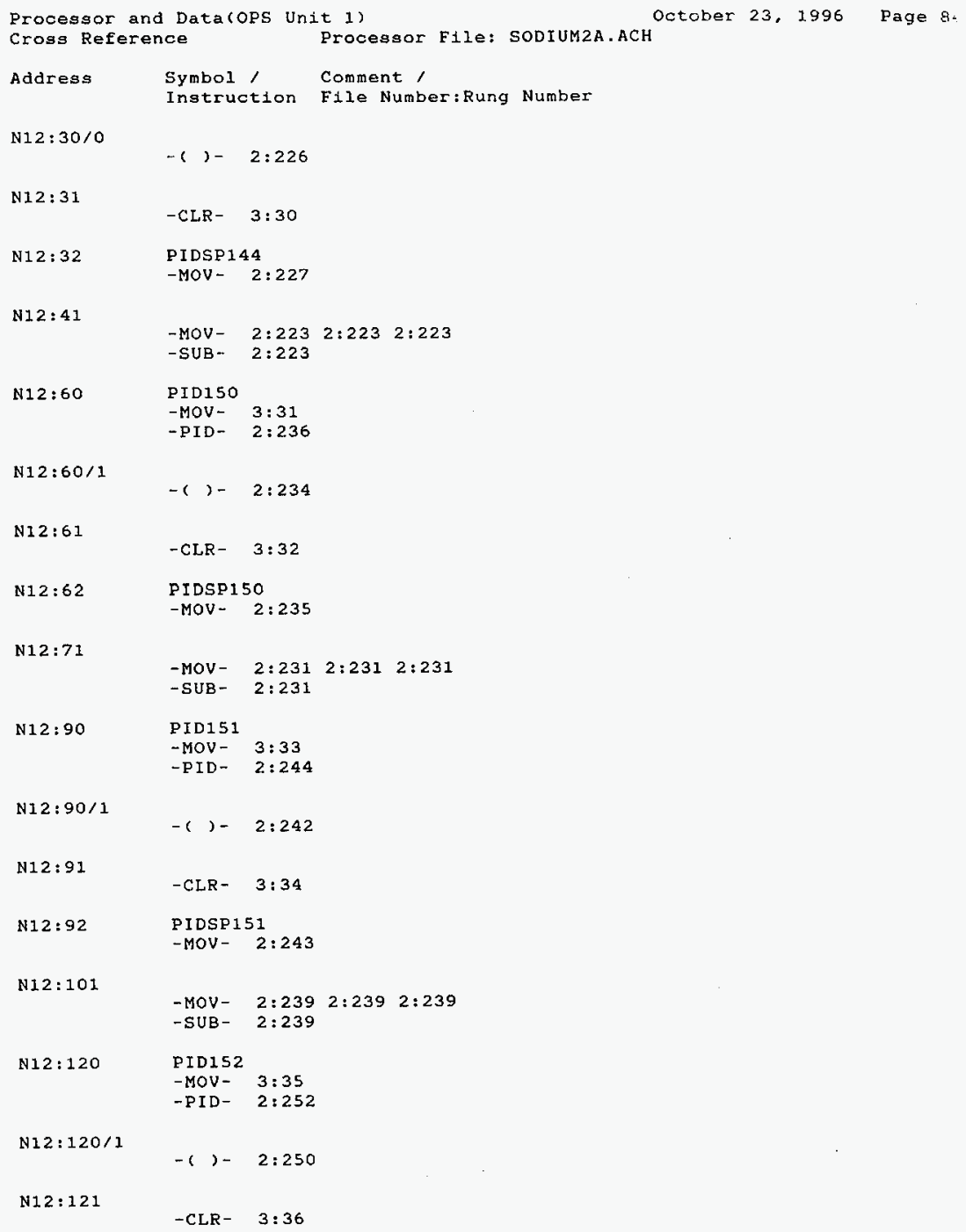

HNF-SD-FF-CSWD-60Rev. 0 
Processor and Data(OPS Unit 1 )

Cross Reference

Address

symbol

Instruction

Comment,

File Number:Rung Number

N12:122

PIDSP152

-MOV- $2: 251$

N12:131

-MOV- 2:247 2:247 2:247

-SUB - 2:247

N12:150

PID153

-MOV- $3: 37$

- PID- 2:260

N12:150/1

-()$-2: 258$

N12:151

-CLR- $3: 38$

N12: 152

PIDSP 153

-MOV- 2:259

N12:161

-MOV- 2:255 2:255 2:255

-SUB- 2:255

N12:180

PID154

-MOV- 3:39

-PID- $2: 268$

N12:180/1

-()$-2: 266$

N12:181

- CLR- 3:40

$\mathrm{N} 12: 182$

PIDSP 154

-MOV- 2:267

N12 : 191

-MOV- 2:263 2:263 2:263

-SUB- 2:263

N13:0

PID160

-MOV- 3:41

-PID- 2:276

$\mathrm{N} 13: 0 / 1$

-()$-2: 274$

N13:1

- CLR- $\quad 3: 42$

$\mathrm{N} 13: 2$

PIDSP160

-MOV- 2:275

$N 13: 11$

-ADD- $2: 271$

$-M O V-2: 271 \quad 2: 271 \quad 2: 271$ 
Processor and Data(OPS Unit 1 )

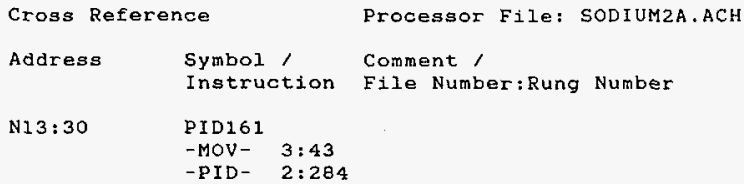

$\mathrm{N} 13: 30 / 1$

-()$-2: 282$

N13:31

-CLR - $\quad 3: 44$

N13:32

PIDSP16I

-MOV- $2: 283$

$\mathrm{N} 13: 41$

-MOV- 2:279 2:279 2:279

-SUB - 2:279

$N 13: 60$

PID162

-MOV- $3: 45$

-PID- $2: 293$

N13:60/1

-()$-2: 291$

N13:61

- CLR- $\quad 3: 46$

$\mathrm{N} 13: 62$

PIDSP162

-MOV- 2:292

N13:71

-MOV- $2: 288 \quad 2: 288 \quad 2: 288$

-SUB- $2: 288$

N1 $3: 90$

PID 163

- MOV- $3: 47$

-PID- 2:456

$N 13: 90 / 1$

-()$-2: 454$

N13: 91

-CLR- $\quad 3: 48$

N13: 92

PIDSP163

-MOV- $2: 455$

N13: 101

-MOV- 2:451 2:451 2:451

-SUB- $2: 45 \mathrm{I}$

N13:120

PID164

-MOV- $3: 49$

-PID- 2:464

N13:120/1

$$
-()-2: 462
$$

HNF-SD-FF-CSWD-60Rev. 0 


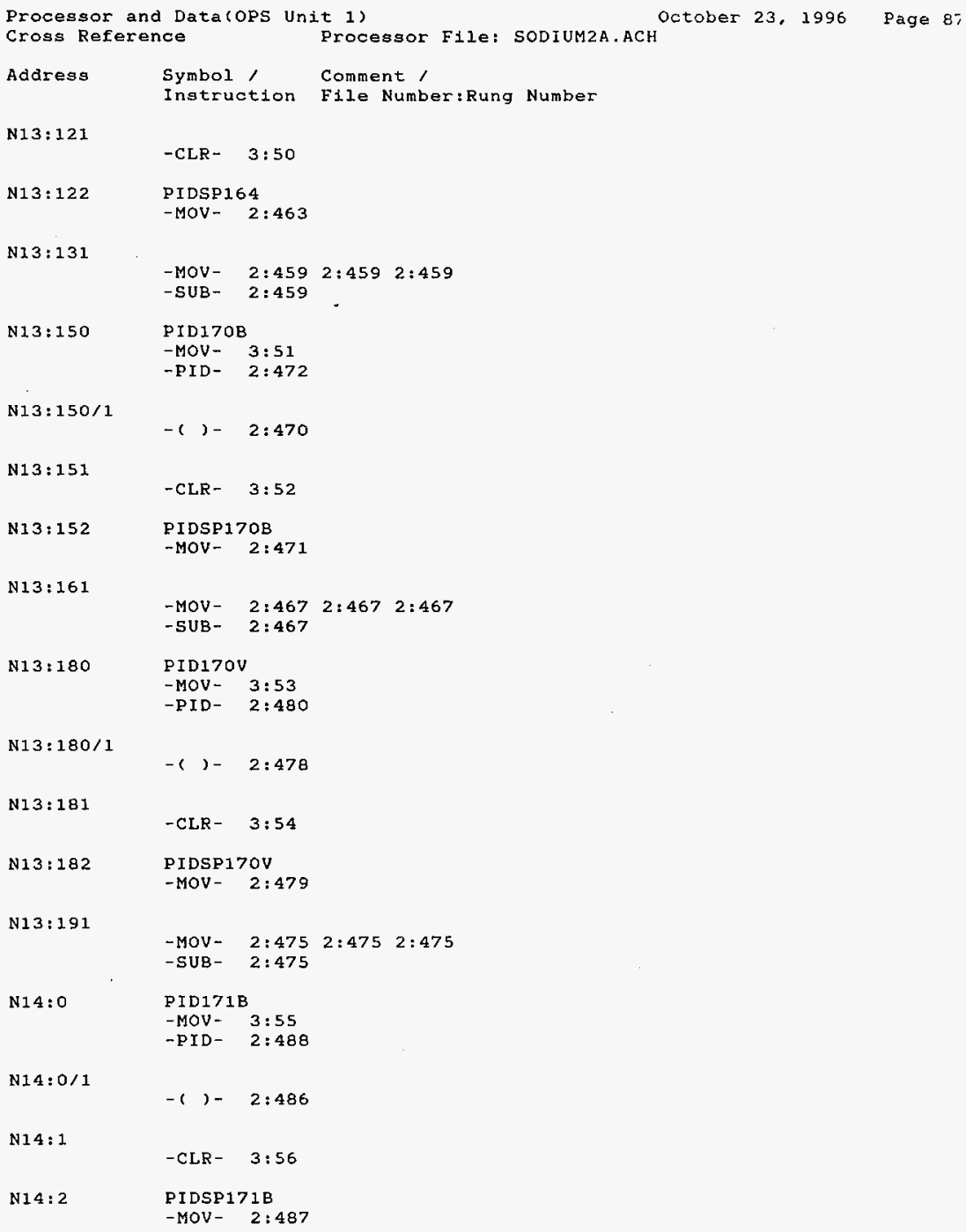

HNF-SD-FF-CSWD-60Rev. 0 
Processor and Data(OPS Unit 1)

Address

Symbol,

Comment ,

Instruction File Number:Rung Number

N14:11

-MOV- $2: 483 \quad 2: 483 \quad 2: 483$

N14:30

PID171V

-MOV- $3: 57$

-PID- 2:496

$N 14: 30 / 1$

-()$-2: 494$

N14:31

-CLR- 3:58

N1 $4: 32$

PIDSP171V

-MOV- 2:495

N14:41

-MOV- 2:491 2:491 2:491

-SUB- 2:483 2:491

N1 $4: 60$

PID $172 \mathrm{~B}$

- MOV - 3:59

-PID- 2:504

N14:60/1

-()$-2: 502$

N14:61

-CLR- 3:60

N1 14:62

PIDSP172B

-MOV 2:503

N14:71

-MOV- 2:499 2:499 2:499

-SUB- 2:499

N14: 90

PIDI72V

-MOV- $3: 61$

-PID- 2:512

N14:90/1

-()$-2: 510$

N1 $4: 91$

-CLR - 3:62

N1 4:92

PIDSP172V

-MOV- 2:511

N14:101

$\begin{array}{llll}-M O V- & 2: 507 \quad 2: 507 & 2: 507\end{array}$

-SUB- 2:507 2:531

N14: 120

PIDI73B

-MOV_ $3: 63$

-PID- 2:520

HNF-SD-FF-CSWD-60Rev. 0 


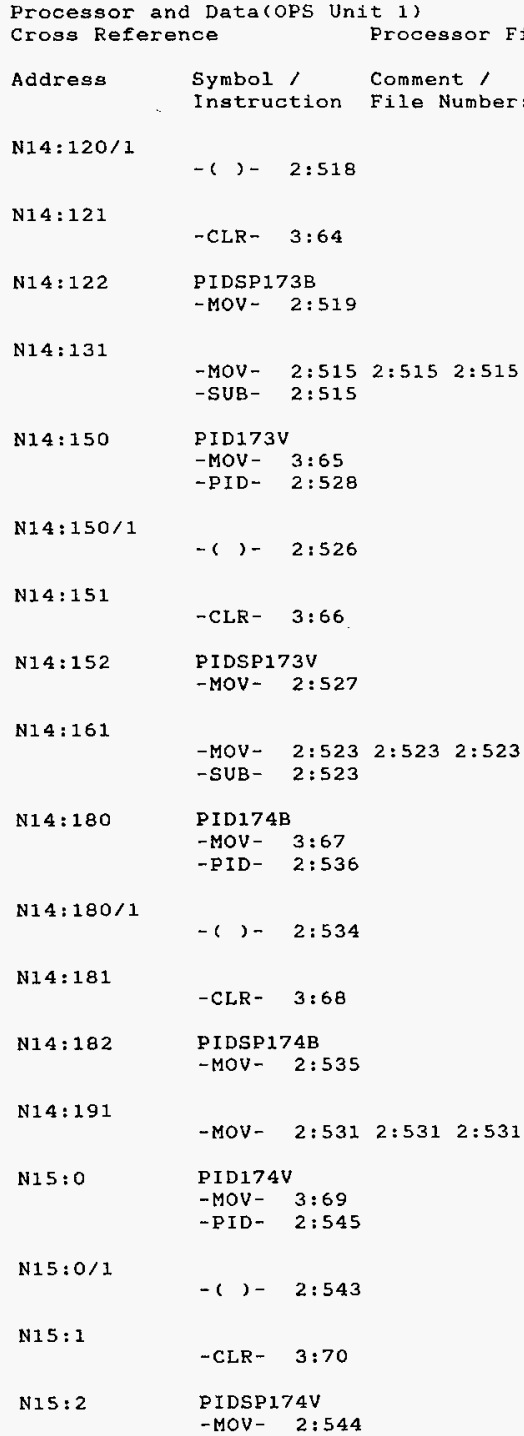

HNF-SD-FF-CSWD-60Rev, 0 
Processor and Data(ops Unit 1 )

October 23, 1996 Page 96

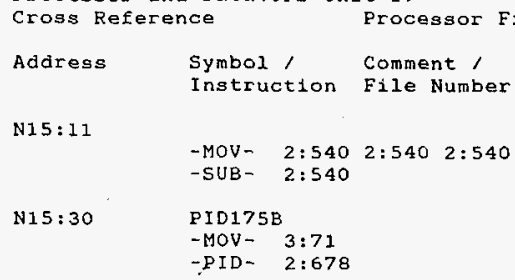

N15: 11

-MOV- 2:540 2:540 2:540

-SUB- 2:540

N15:30

PID175B

-MOV- $3: 71$

-PID- $2: 678$

N15:30/1

-()$-2: 676$

$\mathrm{N} 15: 31$

$-C L R-\quad 3: 72$

N1 $5: 32$

PIDSP175B

-MOV- 2:677

N15:41

-MOV- 2:673 2:673 2:673

-SUB - 2:673

N15:60

PID175V

-MOV- $3: 73$

-PID- 2:686

N15:60/1

-()$-2: 684$

N15:61

-CLR- $\quad 3: 74$

N1 $5: 62$

PIDSP175V

-MOV- 2:685

N15:71

-MOV- 2:681 2:681 2:681

-SUB- 2:681

N15:90

PID176B

-MOV- $3: 75$

-PID- 2:694

N15:90/1

-()$-2: 692$

N15:91

$-C L R-\quad 3: 76$

N15:92

PIDSP176B

-MOV- 2:693

N1 $5: 101$

-MOV- $2: 689 \quad 2: 689 \quad 2: 689 \quad 2: 697 \quad 2: 697 \quad 2: 697$

-SUB- 2:689 2:697

N15:120

PID $176 \mathrm{~V}$

-MOV - $3: 77$

-PID- $2: 702$

HNF-SD-FF-CSWD-60Rev, 0 


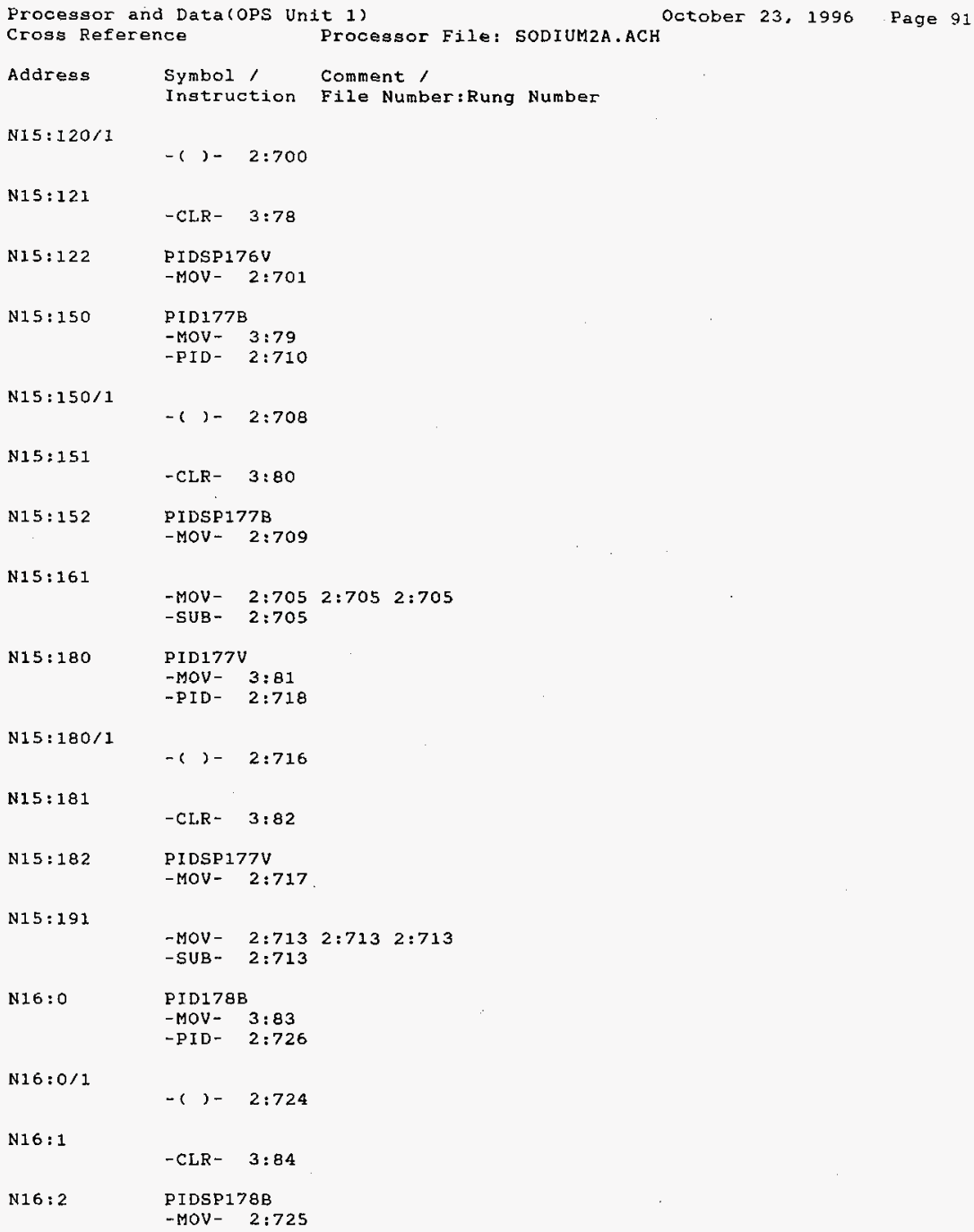

N16:1

-CLR- $\quad 3: 84$

N16:2 PIDSP178B

-MOV- 2:725

HNF-SD-FF-CSWD-6DRev, 0 
Processor and Data(ops Unit I)

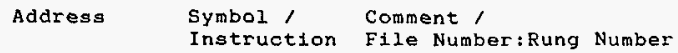

N16:11

MOV- $2: 721 \quad 2: 721 \quad 2: 721$

-SUB- $2: 721$

N16:30

PID178V

-MOV- $3: 85$

-PID- 2:734

N16:30/1

-()$-2: 732$

N16:31

-CLR- $\quad 3: 86$

$N 16: 32$

PIDSP178V

-MOV- $2: 733$

N16:41

-MOV- $2: 729 \quad 2: 729 \quad 2: 729$

-SUB- 2:729

N16:60

PID179B

-MOV- $3: 87$

-PID- $2: 743$

N16:60/1

$-(2)-2: 741$

N16:61

- CLR - $\quad 3: 88$

N16:62

PIDSP179B

-MOV- $\quad 2: 742$

N16:71

-MOV- 2:737 2:737 2:737

-SUB- 2:737

N16:90

PIDI79V

-MOV - $3: 89$

-PID- $2: 876$

N16:90/1

-()$-2: 874$

N16:91

-CLR - $\quad 3: 90$

N16:92

PIDSP179V

-MOV - 2:875

N16:101

-MOV- 2:871 2:871 2:871

-SUB- $2: 871$

N16:120

PIDI80B

-MOV - 3:91

-PID- 2:884

HNF-SD-FF-CSWD-60 Rev. 0 


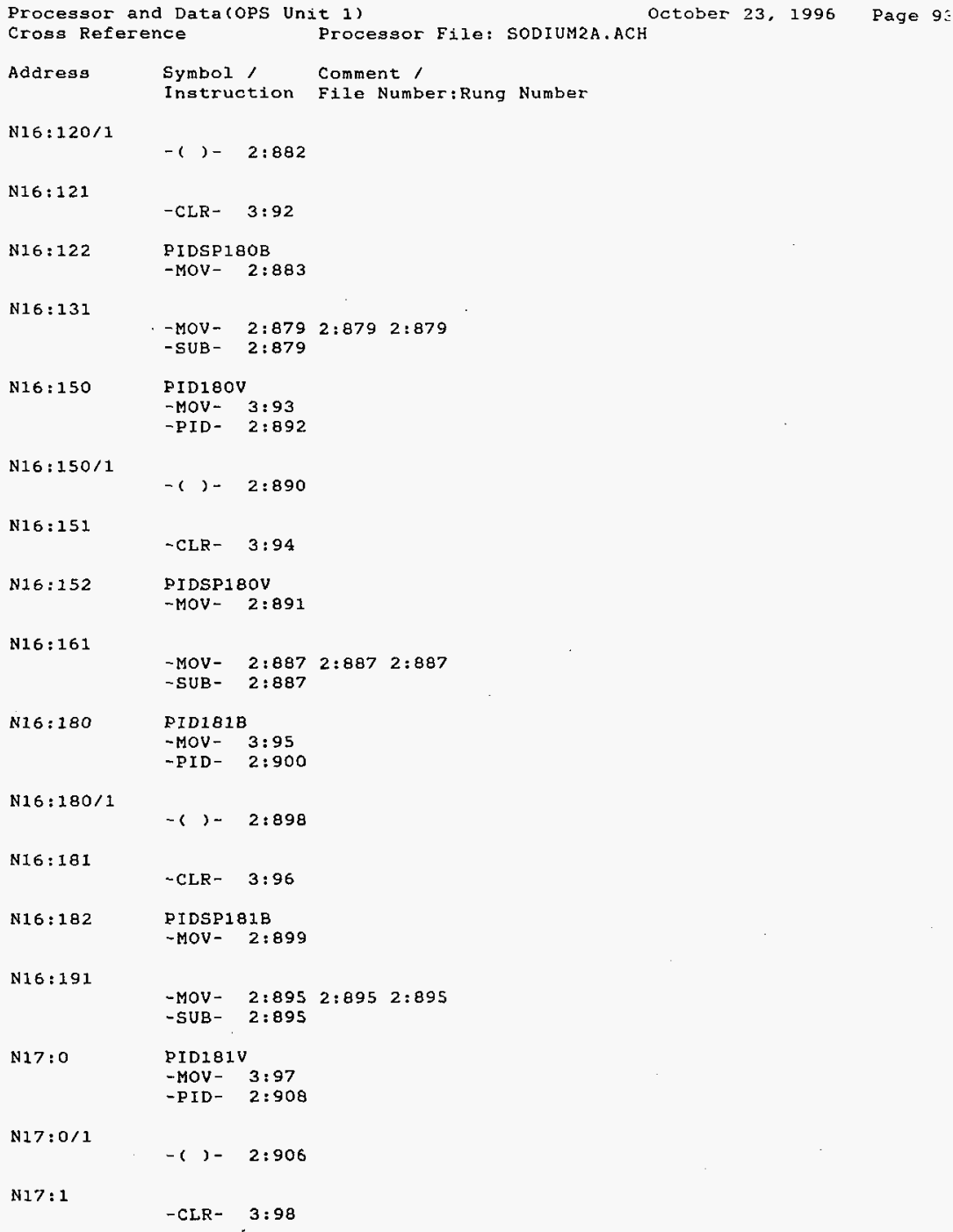

N16:191

-MOV- 2:895 2:895 2:895

-SUB- 2:895

N17:0

PID181V

-MOV- $3: 97$

-PID- 2:908

N17:0/1

$$
-()-2: 906
$$

N17:1

$$
\text { -CLR- 3:98 }
$$




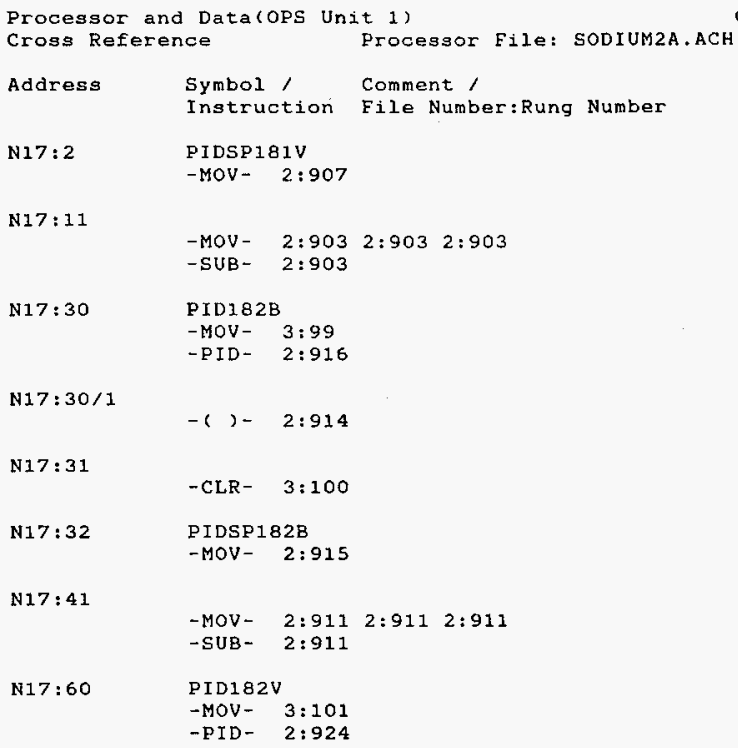

N17:71

-MOV- 2:919 2:919 2:919

- SUB- 2:919

N17:90

PID183B

$-\mathrm{MOV}-\quad 3: 103$

-PID- $2: 932$

N17: $90 / 1$

-()$-2: 930$

N17:91

- CLR- $3: 104$

$\mathrm{N} 17: 92$

PIDSP183B

-MOV- 2:931

N17:101

$\begin{array}{llll}\text { - MOV- } & 2: 927 \quad 2: 927 \quad 2: 927\end{array}$

-SUB- 2:927

HNF-SD-FF-CSWD-6DRev. 0 


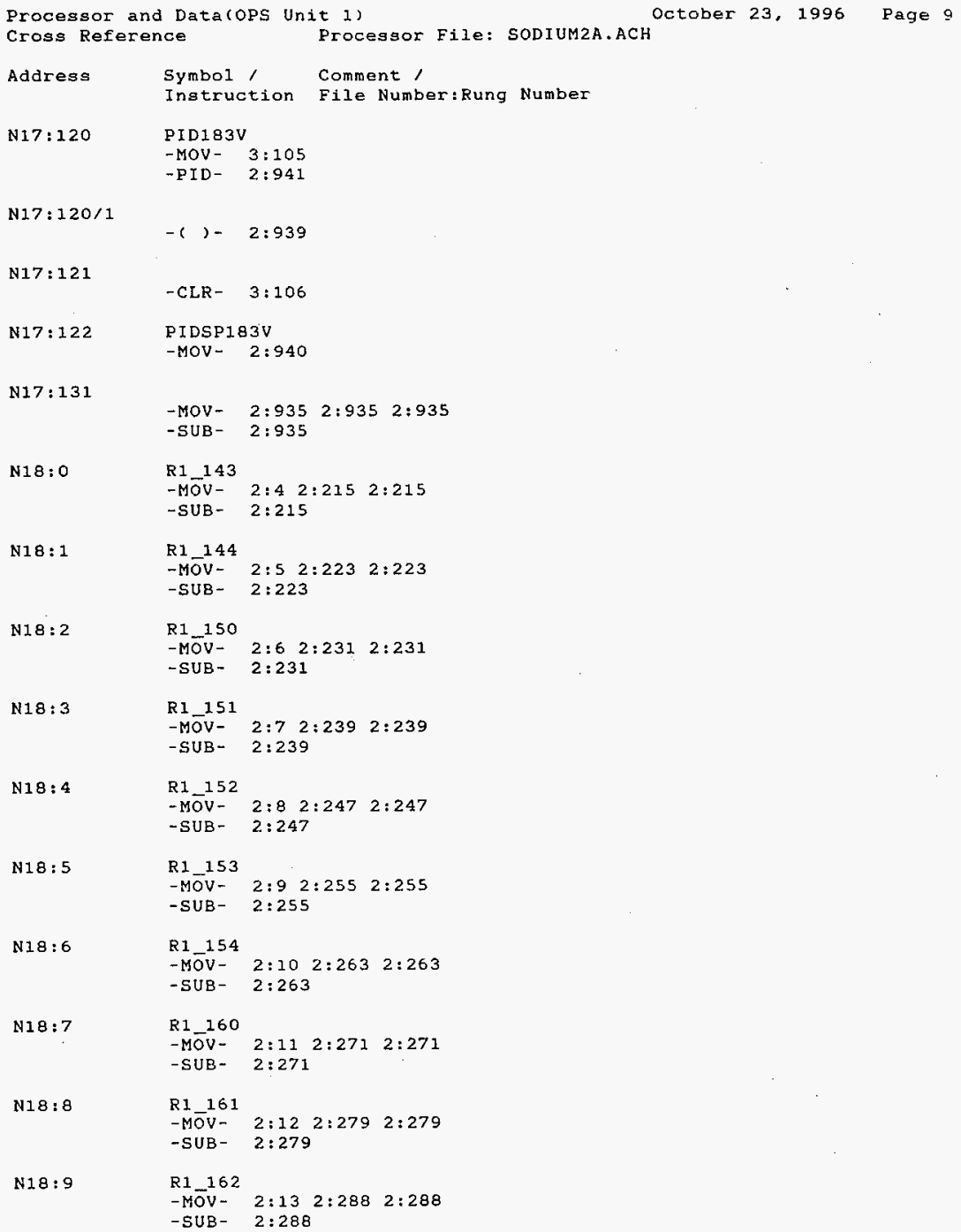

N18:9

R1_162

$\begin{array}{llll}\text {-MOV - } & 2: 13 \quad 2: 288 \quad 2: 288\end{array}$

-SUB- $2: 288$ 


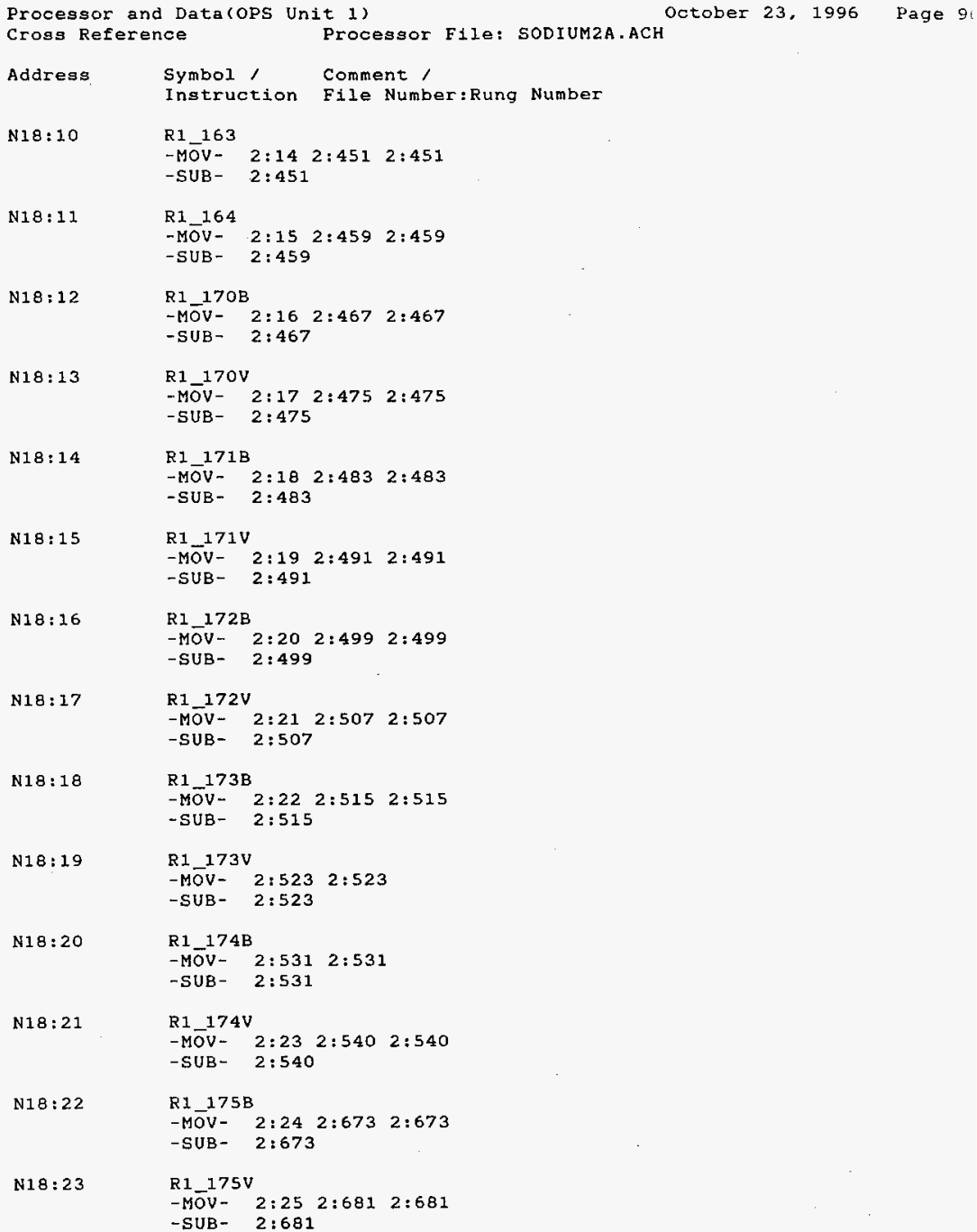

N18:20

R1_174B

-MOV- 2:531 2:531

-SUB- $2: 531$

N18: 21

R1 $174 \mathrm{~V}$

-MOV- 2:23 2:540 2:540

-SUB- 2:540

$\mathrm{N} 18: 22$

R1 175B

-MOV- 2:24 2:673 2:673

-SUB- 2:673

$\mathrm{N} 18: 23$

R1 $175 \mathrm{~V}$

$\begin{array}{lll}\text { MOV- 2:25 2:681 2:681 } & \text { 2:50 }\end{array}$

-SUB- 2:681

HNF-SD-FF-CSWD-60Rev. 0 


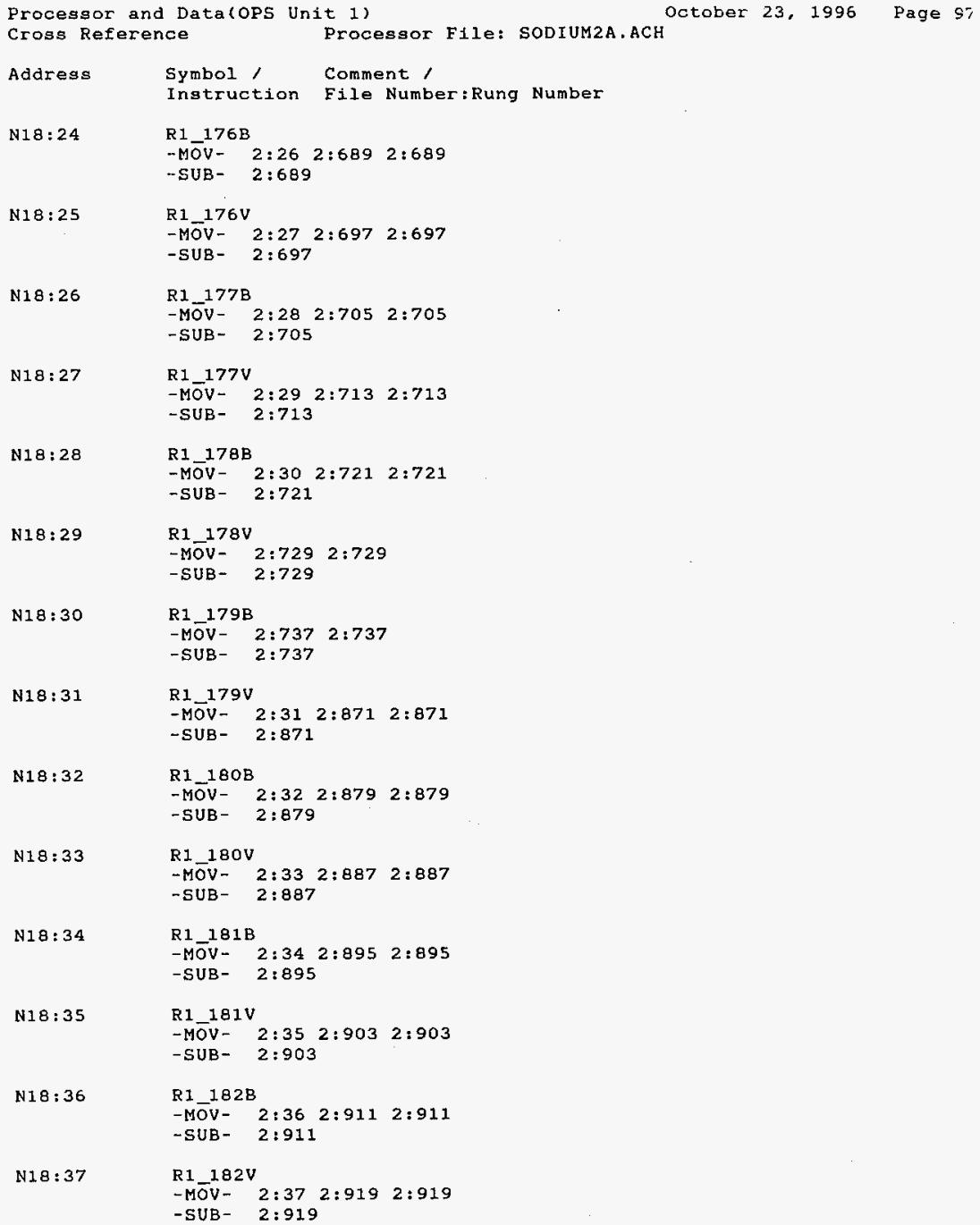

N18:35

R1 $181 \mathrm{~V}$

-MOV- 2:35 2:903 2:903

-SUB- 2:903

N1 $8: 36$

RI $182 \mathrm{~B}$

-MOV- 2:36 2:911 2:911

-SUB- 2:911

$N 18: 37$

R1 $182 \mathrm{~V}$

$\begin{array}{llll}-M \overline{O V}-2: 37 \quad 2: 919 \quad 2: 919 & 2: 9\end{array}$

-SUB- 2:919 


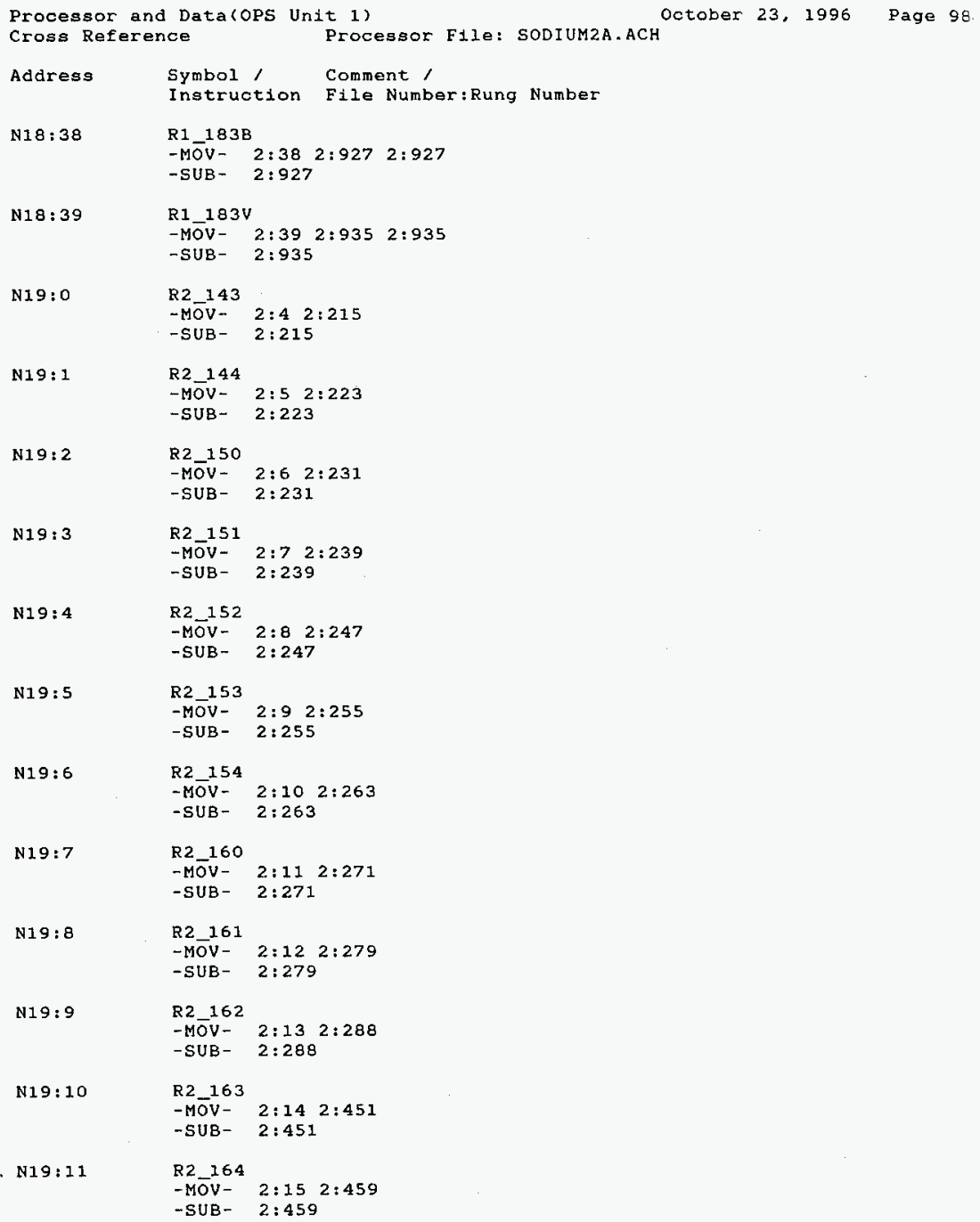




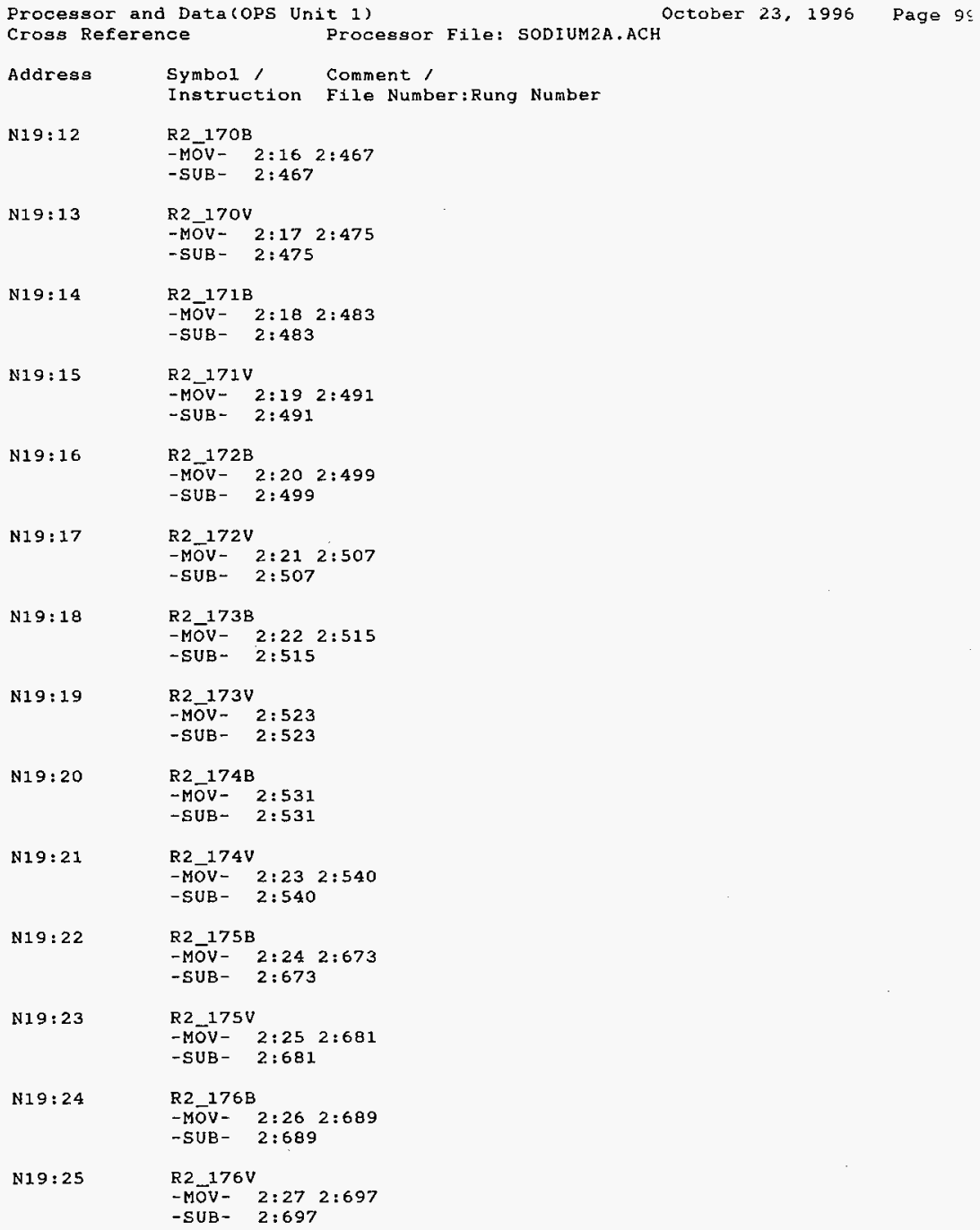

N19:19

R2 $173 \mathrm{~V}$

-MOV- 2:523

$-\mathrm{SUB}-\quad 2: 523$

N19:20

R2 $174 \mathrm{~B}$

MOV- 2:531

-SUB- $2: 531$

N1 $9: 21$

R2 $174 \mathrm{~V}$

MOV- 2:23 2:540

-SUB- 2:540

N19:22

R2 $175 \mathrm{~B}$

-MOV- 2:24 2:673

-SUB- 2:673

$N 19: 23$

R2 $175 \mathrm{~V}$

MOV- 2:25 2:681

-SUB - 2:681

$N 19: 24$

R2_176B

MOV- 2:26 2:689

-SUB- 2:689

N19: 25

R2 $176 \mathrm{~V}$

-MOV- 2:27 2:697

-SUB- 2:697

HNF-SD-FF-CSWD-6 DRev. 0 


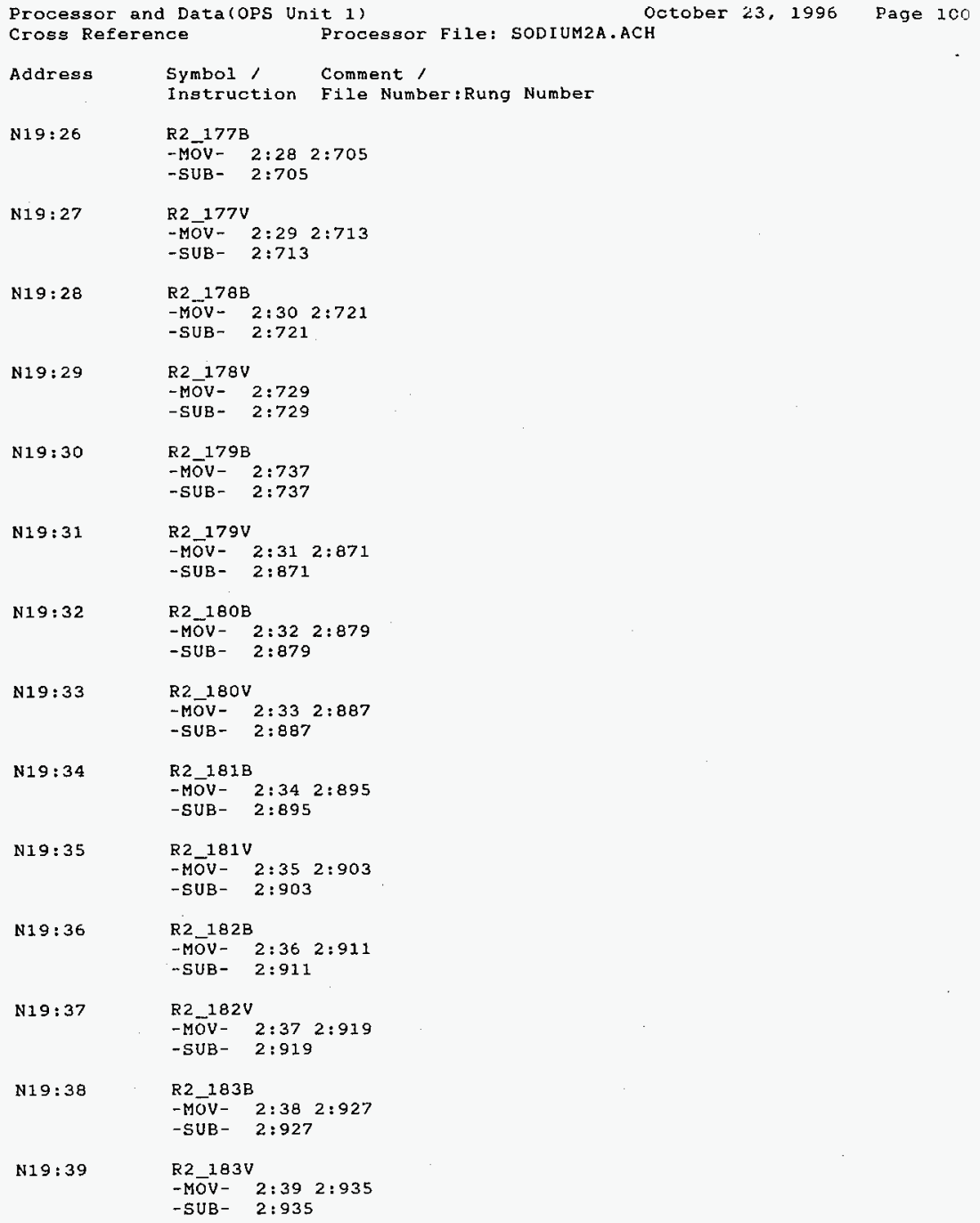

N1 9:38

R2 183B

-MOV- $2: 38 \quad 2: 927$

-SUB- 2:927

N19:39

R2 $183 \mathrm{~V}$

-MOV- 2:39 2:935

-SUB- 2:935

HNF-SD-FF-CSWD-60Rev. 0 


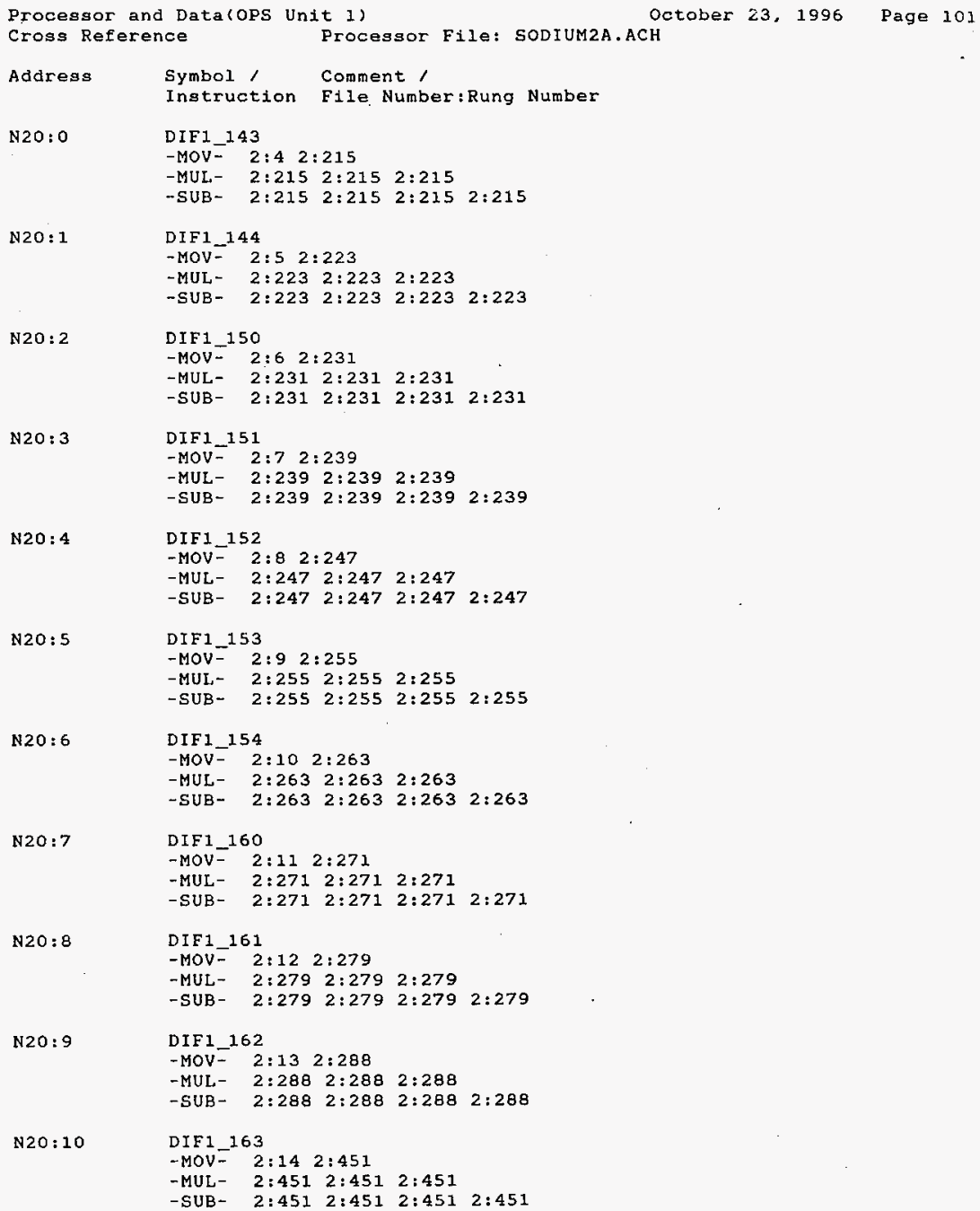

N2O: 10

DIF1_163

MOV= 2:14 2:451

-MUL- 2:451 2:451 2:451

-SUB- 2:451 2:451 2:451 2:451 


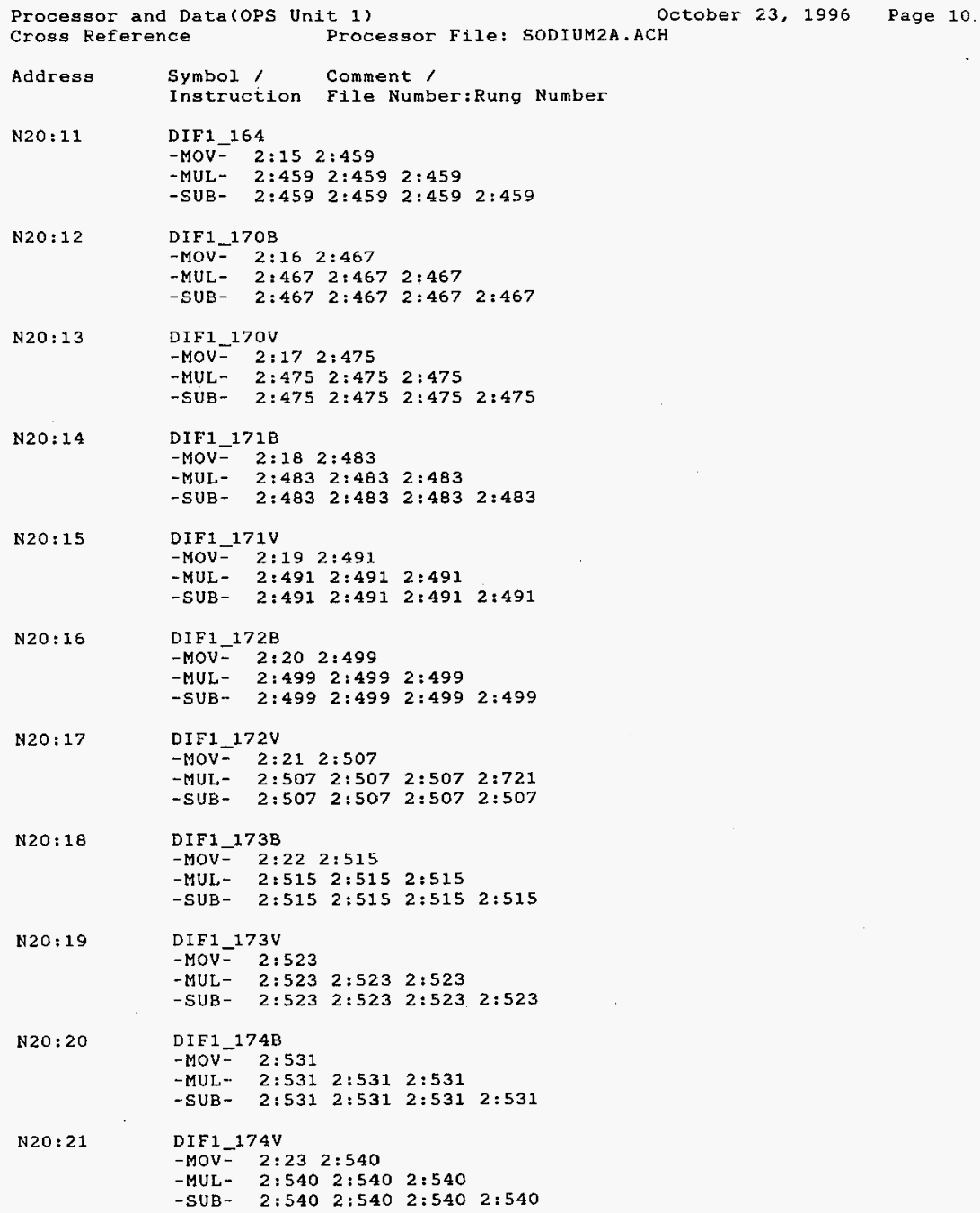




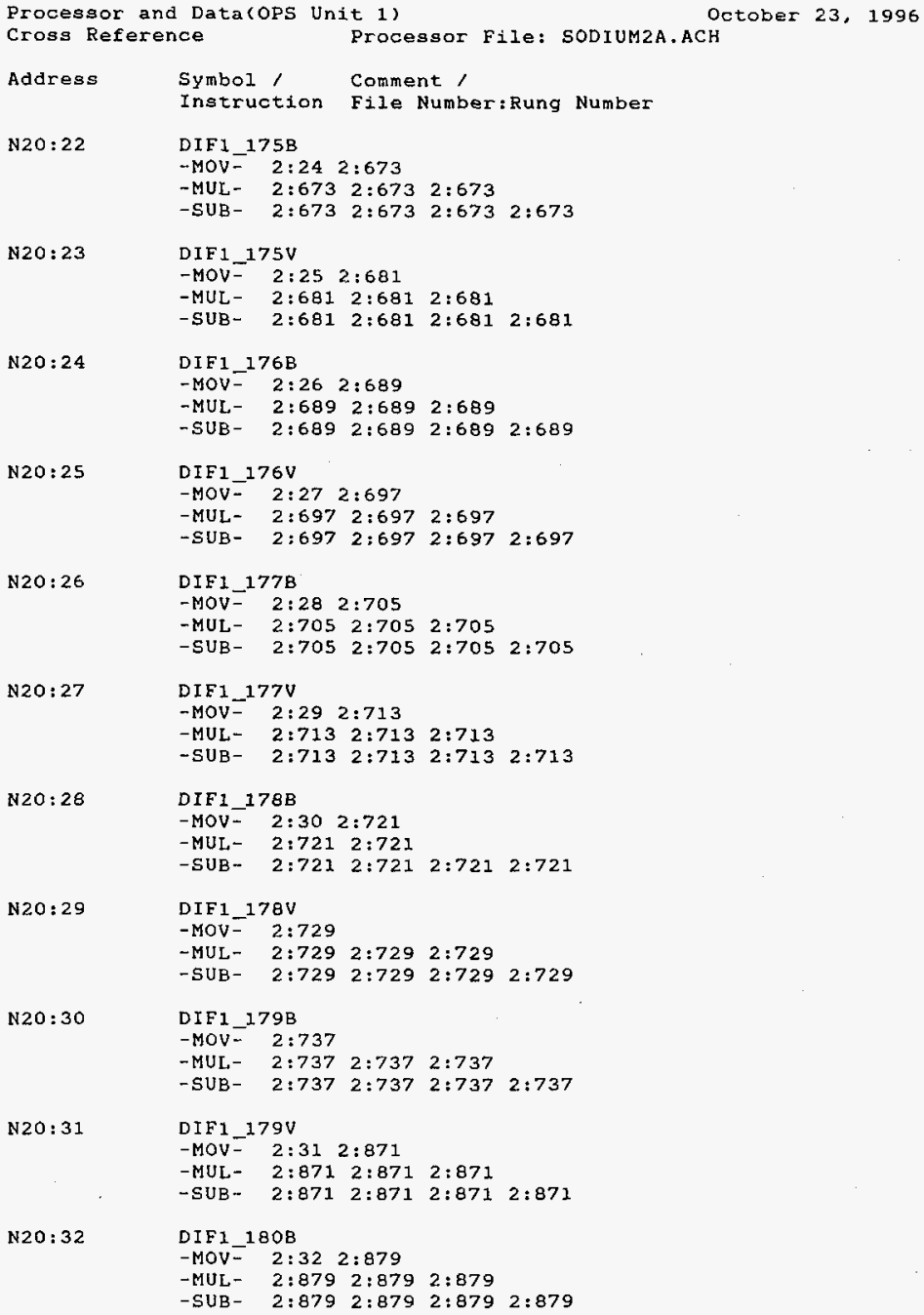

$N 20: 30$

DIF1_ $179 \mathrm{~B}$

- MOV $=2: 737$

-MUL- $2: 737 \quad 2: 737 \quad 2: 737$

$\begin{array}{llll}\text {-SUB- } & 2: 737 \quad 2: 737 \quad 2: 737 \quad 2: 737\end{array}$

$\mathrm{N} 20: 31$

DIF1_179V

-MOV= 2:31 2:871

-MUL- 2:871 2:871 2:871

$\begin{array}{lllll}\text {-SUB - } & 2: 871 & 2: 871 & 2: 871 & 2: 871\end{array}$

N20:32

DIF1 1808

-MOV- $2: 32 \quad 2: 879$

-MUL- $2: 879 \quad 2: 879 \quad 2: 879$

$\begin{array}{lllll}\text {-SUB- } & 2: 879 & 2: 879 & 2: 879 & 2: 879\end{array}$

HNF-SD-FF-CSWD-6ORev. 0 
Processor and Data(OPS Unit 1 )

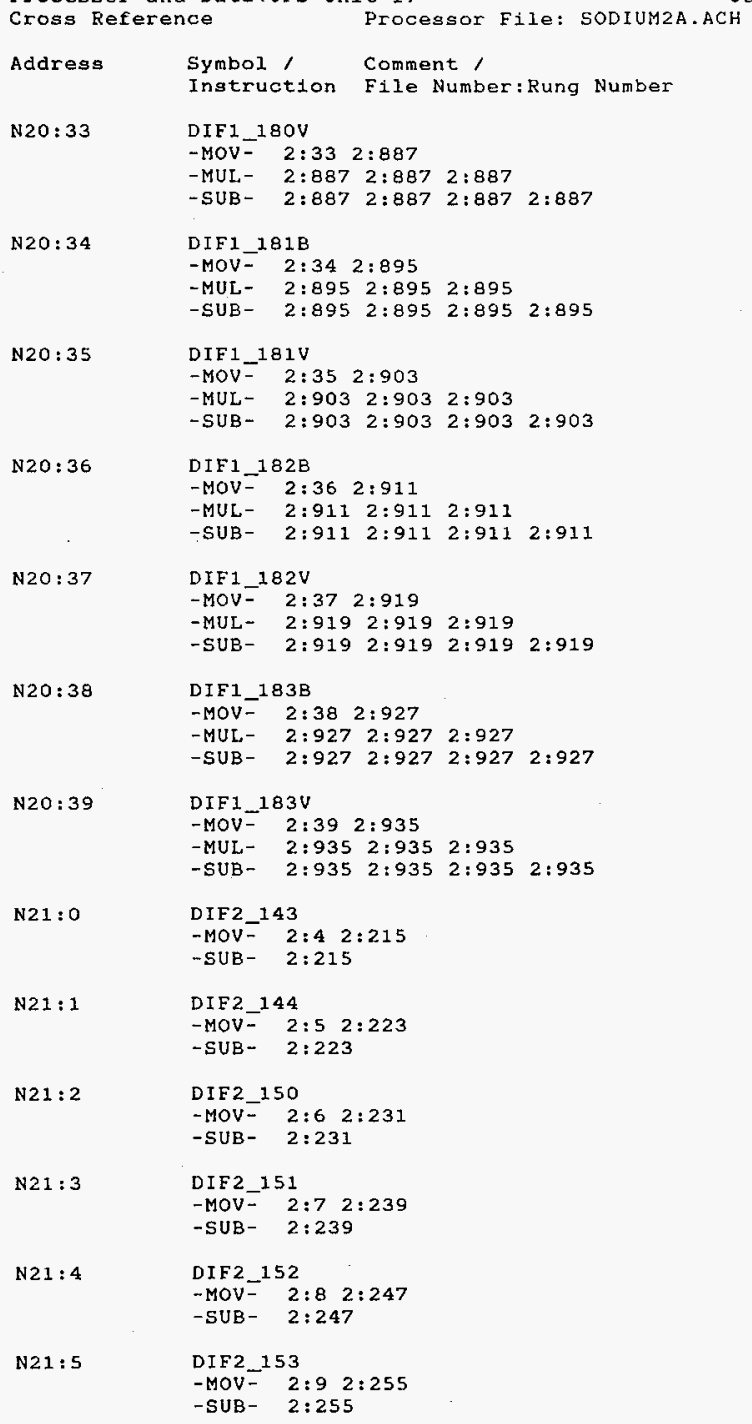

N21: 5 


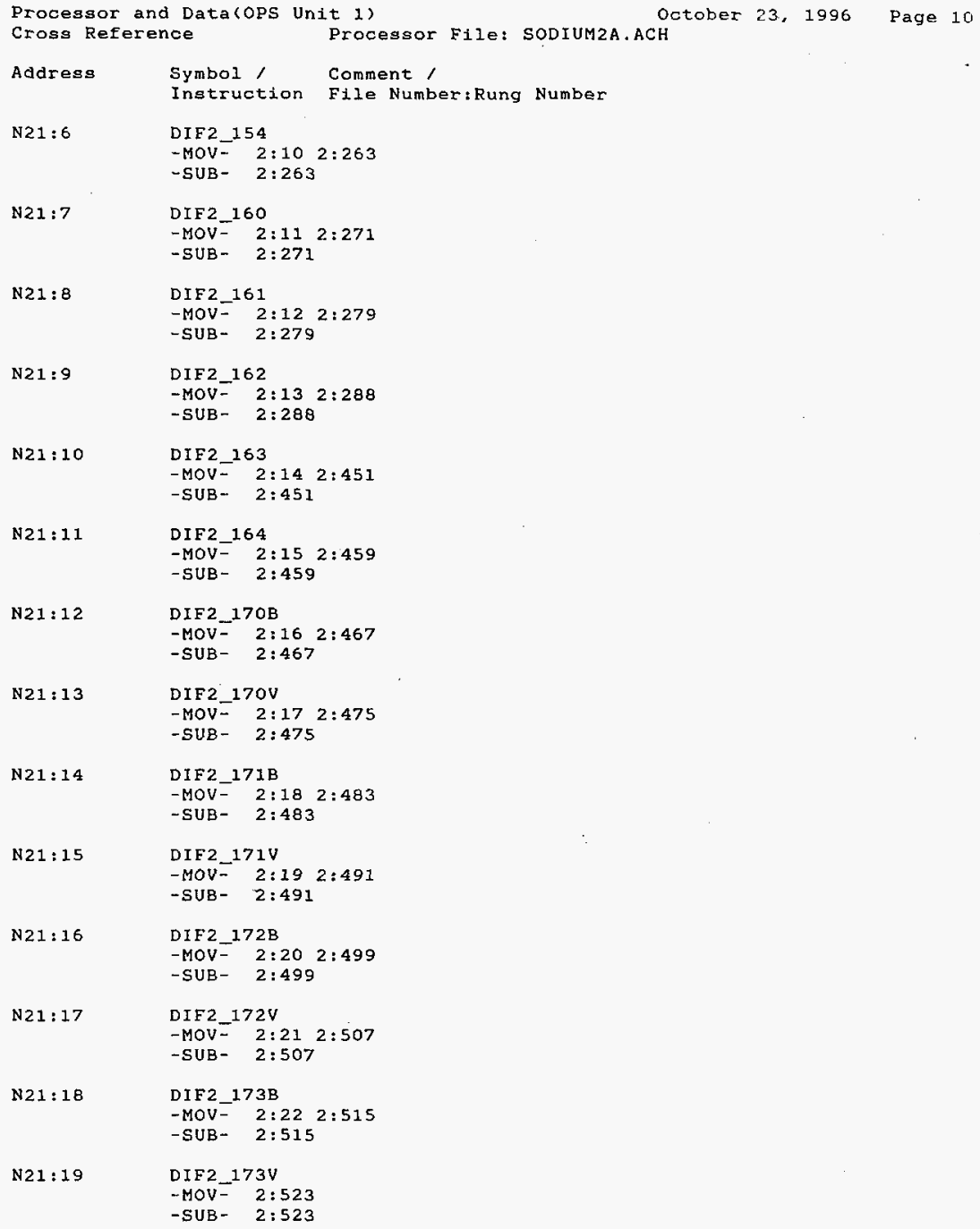




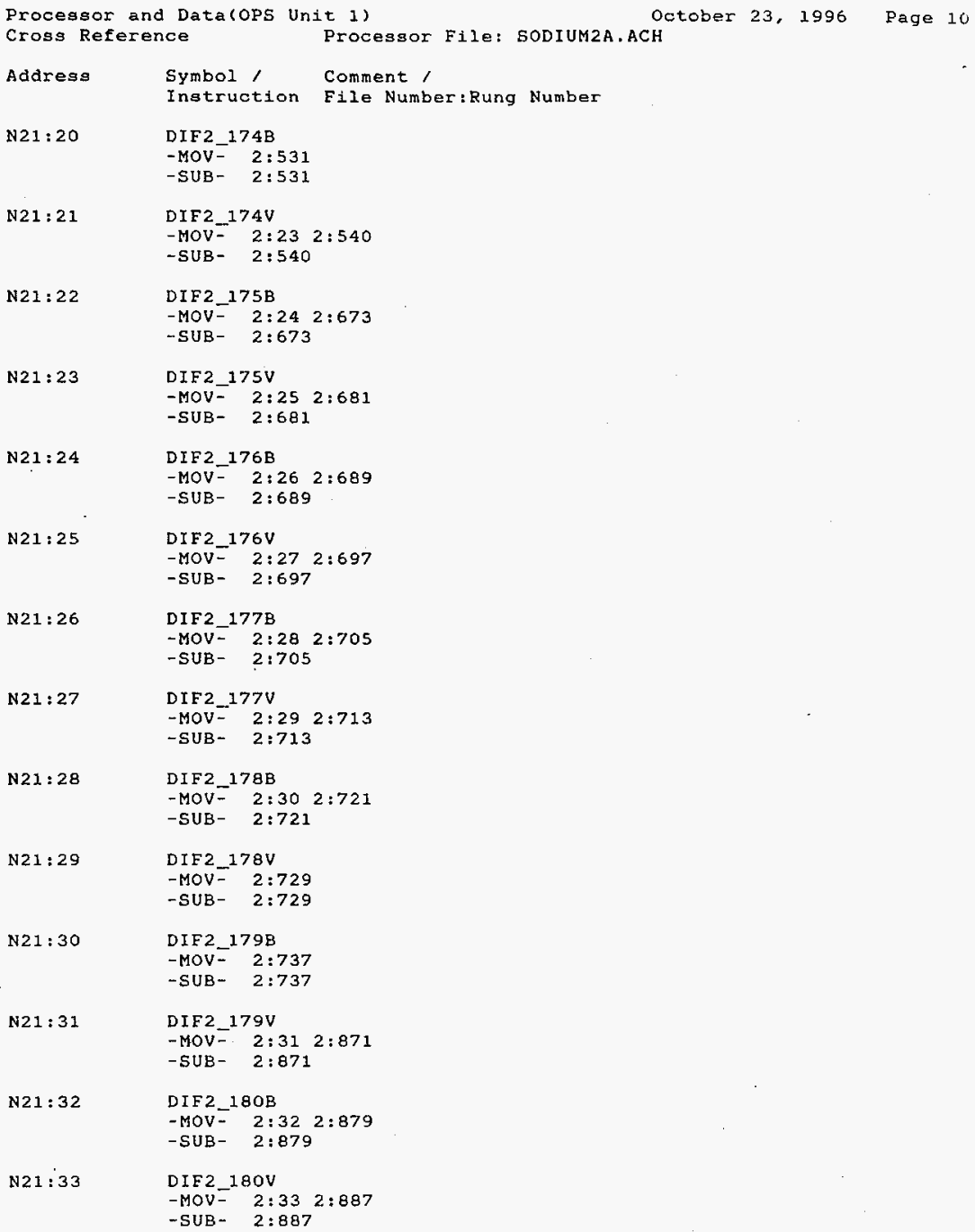


Processor and Data(OPS Unit 1 )

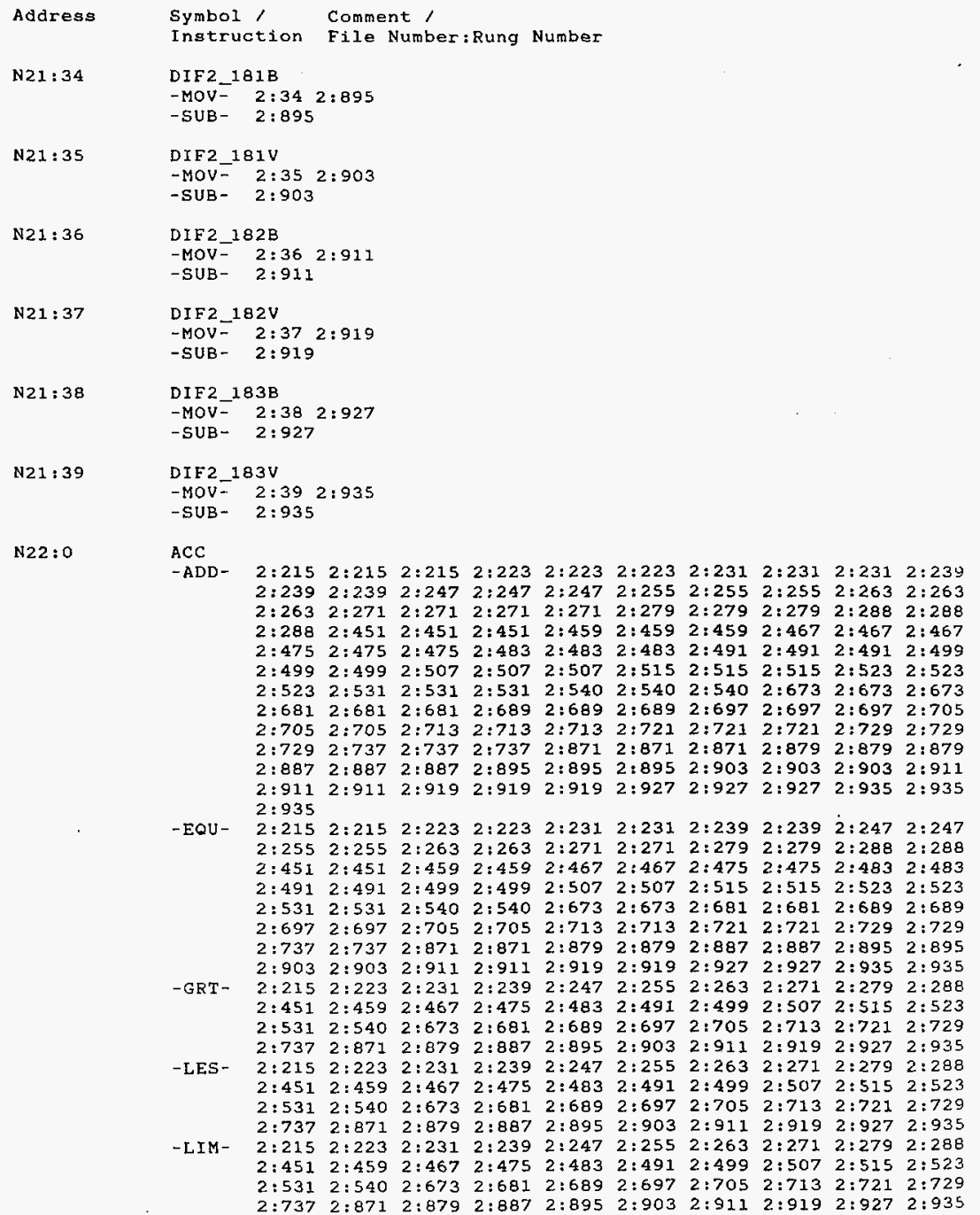

HNF-SD-FF-CSWD-60Rev. 0 
Processor and Data(OPS Unit 1) Cross Reference

Address

Symbol,

Comment ,

Instruction File Number:Rung Number

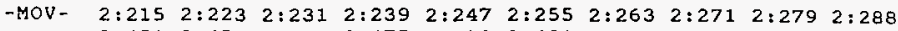
$\begin{array}{llllllllll}2: 451 & 2: 459 & 2: 467 & 2: 475 & 2: 483 & 2: 491 & 2: 499 & 2: 507 & 2: 515 & 2: 523\end{array}$

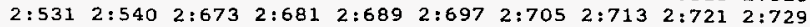
$\begin{array}{llllllllll}2: 737 & 2: 871 & 2: 879 & 2: 887 & 2: 895 & 2: 903 & 2: 911 & 2: 919 & 2: 927 & 2: 935\end{array}$

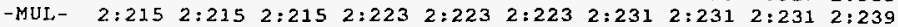
$\begin{array}{llllllllll}2: 239 & 2: 239 & 2: 247 & 2: 247 & 2: 247 & 2: 255 & 2: 255 & 2: 255 & 2: 263 & 2: 263\end{array}$

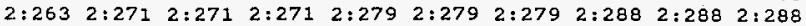
$\begin{array}{lllllllllll}2: 451 & 2: 451 & 2: 451 & 2: 459 & 2: 459 & 2: 459 & 2: 467 & 2: 467 & 2: 467 & 2: 475\end{array}$

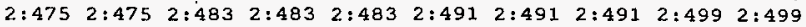
$\begin{array}{llllllllll}2: 499 & 2: 507 & 2: 507 & 2: 507 & 2: 515 & 2: 515 & 2: 515 & 2: 523 & 2: 523 & 2: 523\end{array}$

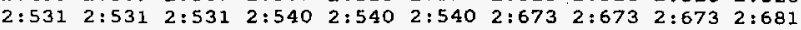
$\begin{array}{llllllllll}2: 681 & 2: 681 & 2: 689 & 2: 689 & 2: 689 & 2: 697 & 2: 697 & 2: 697 & 2: 705 & 2: 705\end{array}$ $\begin{array}{llllllllll}2: 705 & 2: 713 & 2: 713 & 2: 713 & 2: 721 & 2: 721 & 2: 721 & 2: 729 & 2: 729 & 2: 729\end{array}$ $\begin{array}{lllllllllll}2: 737 & 2: 737 & 2: 737 & 2: 871 & 2: 871 & 2: 871 & 2: 879 & 2: 879 & 2: 879 & 2: 887\end{array}$ $\begin{array}{llllllllll}2: 887 & 2: 887 & 2: 895 & 2: 895 & 2: 895 & 2: 903 & 2: 903 & 2: 903 & 2: 911 & 2: 911\end{array}$

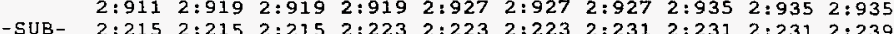

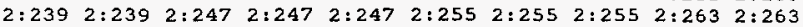

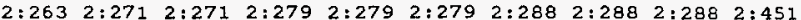

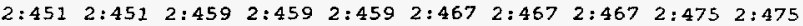

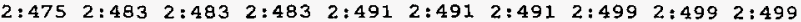

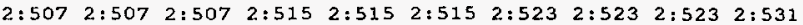
$\begin{array}{lllllllllll}2: 531 & 2: 531 & 2: 540 & 2: 540 & 2: 540 & 2: 673 & 2: 673 & 2: 673 & 2: 681 & 2: 681\end{array}$ $\begin{array}{lllllllllll}2: 681 & 2: 689 & 2: 689 & 2: 689 & 2: 697 & 2: 697 & 2: 697 & 2: 705 & 2: 705 & 2: 705\end{array}$ $\begin{array}{llllllllllll}2: 713 & 2: 713 & 2: 713 & 2: 721 & 2: 721 & 2: 721 & 2: 729 & 2: 729 & 2: 729 & 2: 737\end{array}$

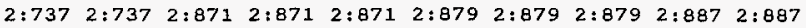
$\begin{array}{lllllllllll}2: 887 & 2: 895 & 2: 895 & 2: 895 & 2: 903 & 2: 903 & 2: 903 & 2: 911 & 2: 911 & 2: 911\end{array}$

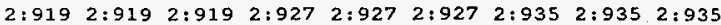

N22:1 ACC1

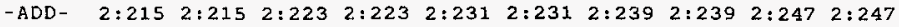

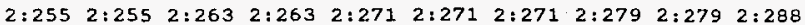

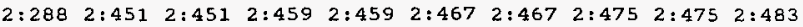

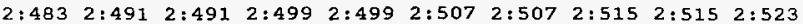
$\begin{array}{llllllllll}2: 523 & 2: 531 & 2: 531 & 2: 540 & 2: 540 & 2: 673 & 2: 673 & 2: 681 & 2: 681 & 2: 689\end{array}$ $\begin{array}{lllllllllll}2: 689 & 2: 697 & 2: 697 & 2: 705 & 2: 705 & 2: 713 & 2: 713 & 2: 721 & 2: 721 & 2: 729\end{array}$ $\begin{array}{llllllllll}2: 729 & 2: 737 & 2: 737 & 2: 871 & 2: 871 & 2: 879 & 2: 879 & 2: 887 & 2: 887 & 2: 895\end{array}$

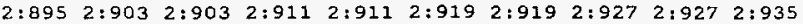
$2: 935$

-MUL- $2: 215 \quad 2: 215 \quad 2: 215 \quad 2: 223 \quad 2: 223 \quad 2: 223 \quad 2: 231 \quad 2: 231 \quad 2: 231 \quad 2: 239$

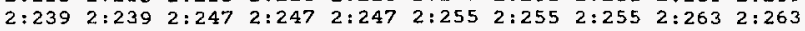
$\begin{array}{llllllllll}2: 263 & 2: 271 & 2: 271 & 2: 271 & 2: 279 & 2: 279 & 2: 279 & 2: 288 & 2: 288 & 2: 288\end{array}$

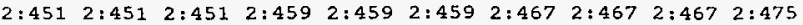
$\begin{array}{llllllllll}2: 475 & 2: 475 & 2: 483 & 2: 483 & 2: 483 & 2: 491 & 2: 491 & 2: 491 & 2: 499 & 2: 499\end{array}$

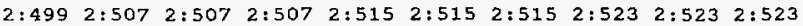
$\begin{array}{llllllllll}2: 531 & 2: 531 & 2: 531 & 2: 540 & 2: 540 & 2: 540 & 2: 673 & 2: 673 & 2: 673 & 2: 681\end{array}$ $\begin{array}{llllllllll}2: 681 & 2: 681 & 2: 689 & 2: 689 & 2: 689 & 2: 697 & 2: 697 & 2: 697 & 2: 705 & 2: 705\end{array}$ $\begin{array}{llllllllll}2: 705 & 2: 713 & 2: 713 & 2: 713 & 2: 721 & 2: 721 & 2: 721 & 2: 729 & 2: 729 & 2: 729\end{array}$ $\begin{array}{llllllllll}2: 737 & 2: 737 & 2: 737 & 2: 871 & 2: 871 & 2: 871 & 2: 879 & 2: 879 & 2: 879 & 2: 887\end{array}$

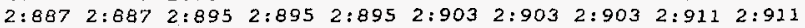
$\begin{array}{llllllllll}2: 911 & 2: 919 & 2: 919 & 2: 919 & 2: 927 & 2: 927 & 2: 927 & 2: 935 & 2: 935 & 2: 935\end{array}$

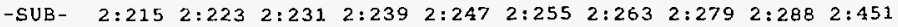
$\begin{array}{llllllllll}2: 459 & 2: 467 & 2: 475 & 2: 483 & 2: 491 & 2: 499 & 2: 507 & 2: 515 & 2: 523 & 2: 531\end{array}$ $\begin{array}{lllllllllll}2: 540 & 2: 673 & 2: 681 & 2: 689 & 2: 697 & 2: 705 & 2: 713 & 2: 721 & 2: 729 & 2: 737\end{array}$

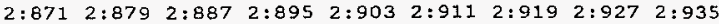

HNF-SD-FF-CSWD-6DRev. 0 
Processor and Data(OPS Unit 1) Processor File: SODIUM2A.ACH

October 23, 1996 Page 10 Cross Reference

$\begin{array}{ll}\text { Address Symbol, } & \text { Comment } / \\ & \text { Instruction File Number:Rung Number }\end{array}$

N22:2 DDIF1

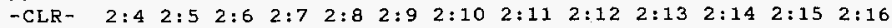

$\begin{array}{llllllllllll}2: 17 & 2: 18 & 2: 19 & 2: 20 & 2: 21 & 2: 22 & 2: 23 & 2: 24 & 2: 25 & 2: 26 & 2: 27 & 2: 28\end{array}$ $\begin{array}{lllllllllll}2: 29 & 2: 30 & 2: 31 & 2: 32 & 2: 33 & 2: 34 & 2: 35 & 2: 36 & 2: 37 & 2: 38 & 2: 39\end{array}$

-MUL- $2: 215 \quad 2: 215 \quad 2: 215 \quad 2: 223 \quad 2: 223 \quad 2: 223 \quad 2: 231 \quad 2: 231 \quad 2: 231 \quad 2: 239$ $\begin{array}{llllllllll}2: 239 & 2: 239 & 2: 247 & 2: 247 & 2: 247 & 2: 255 & 2: 255 & 2: 255 & 2: 263 & 2: 263\end{array}$

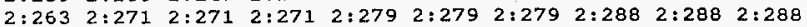
$\begin{array}{lllllllllll}2: 451 & 2: 451 & 2: 451 & 2: 459 & 2: 459 & 2: 459 & 2: 467 & 2: 467 & 2: 467 & 2: 475\end{array}$

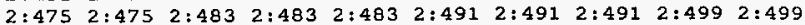
$\begin{array}{llllllllll}2: 499 & 2: 507 & 2: 507 & 2: 507 & 2: 515 & 2: 515 & 2: 515 & 2: 523 & 2: 523 & 2: 523\end{array}$ $\begin{array}{llllllllll}2: 531 & 2: 531 & 2: 531 & 2: 540 & 2: 540 & 2: 540 & 2: 673 & 2: 573 & 2: 673 & 2: 681\end{array}$ $\begin{array}{llllllllll}2: 681 & 2: 681 & 2: 689 & 2: 689 & 2: 689 & 2: 697 & 2: 697 & 2: 697 & 2: 705 & 2: 705\end{array}$ $\begin{array}{llllllllll}2: 705 & 2: 713 & 2: 713 & 2: 713 & 2: 721 & 2: 721 & 2: 721 & 2: 729 & 2: 729 & 2: 729\end{array}$ $\begin{array}{lllllllllll}2: 737 & 2: 737 & 2: 737 & 2: 871 & 2: 871 & 2: 871 & 2: 879 & 2: 879 & 2: 879 & 2: 887\end{array}$ $\begin{array}{llllllllll}2: 887 & 2: 887 & 2: 895 & 2: 895 & 2: 895 & 2: 903 & 2: 903 & 2: 903 & 2: 911 & 2: 911\end{array}$ $\begin{array}{llllllllll}2: 911 & 2: 919 & 2: 919 & 2: 919 & 2: 927 & 2: 927 & 2: 927 & 2: 935 & 2: 935 & 2: 935\end{array}$

-SUB- $2: 215 \quad 2: 223 \quad 2: 231 \quad 2: 239 \quad 2: 247 \quad 2: 255 \quad 2: 263 \quad 2: 271 \quad 2: 279 \quad 2: 288$

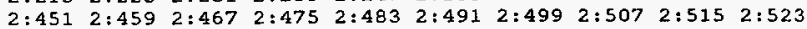
$\begin{array}{llllllllll}2: 531 & 2: 540 & 2: 673 & 2: 681 & 2: 689 & 2: 697 & 2: 705 & 2: 713 & 2: 721 & 2: 729\end{array}$

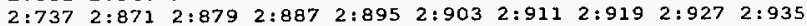

N22: 3

DDIF2

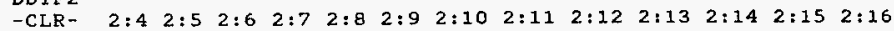

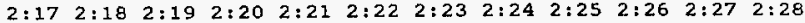

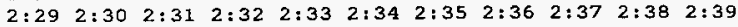

$\mathrm{N} 22: 5 / 1$

DN_1

$-]-2: 43$

$-] /[-2: 44$

$-(\mathrm{L})-2: 50$

-(U) - 2:40 2:43

N22:5/2

DN_2

$-][-2: 43$

$-] /[-2: 45$

$-(L)-2: 296$

$-(U)-2: 402: 43$

N22:5/3

DN_3

$-{ }^{-}[-$

$2: 43$

$-] /\left[\begin{array}{ll}-146 & 2: 67]\end{array}\right.$

$-(L)-2: 548$

-(U) - $2: 40 \quad 2: 40 \quad 2: 43$

N22:5/4

DN_4

$-]$ [ -

$-] /[-$

$2: 43$

- (L) - 2:746

- (U) - 2:43

N22:5/5 DNR_1

$$
\begin{array}{ll}
\text {-] } \bar{L}- & 2: 43 \\
-(L)- & 2: 287 \\
-(U)- & 2: 43
\end{array}
$$

HNF-SD-FF-CSWD-60Rev. 0 
Processor and Data(OPS Unit 1)

October 23, 1996 Page I1

Cross Reference

Processor File: SODIUM $2 \mathrm{~A} . \mathrm{ACH}$

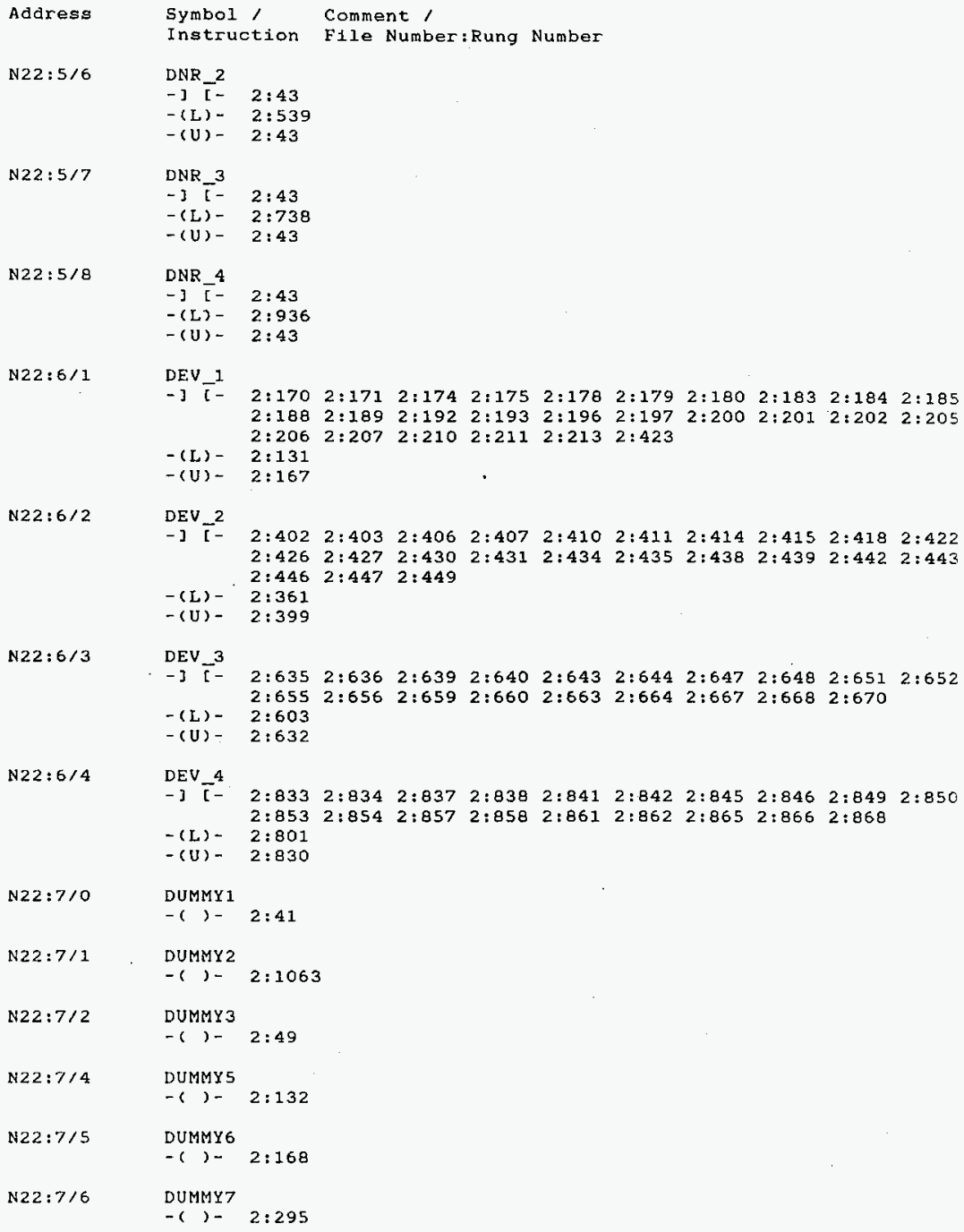




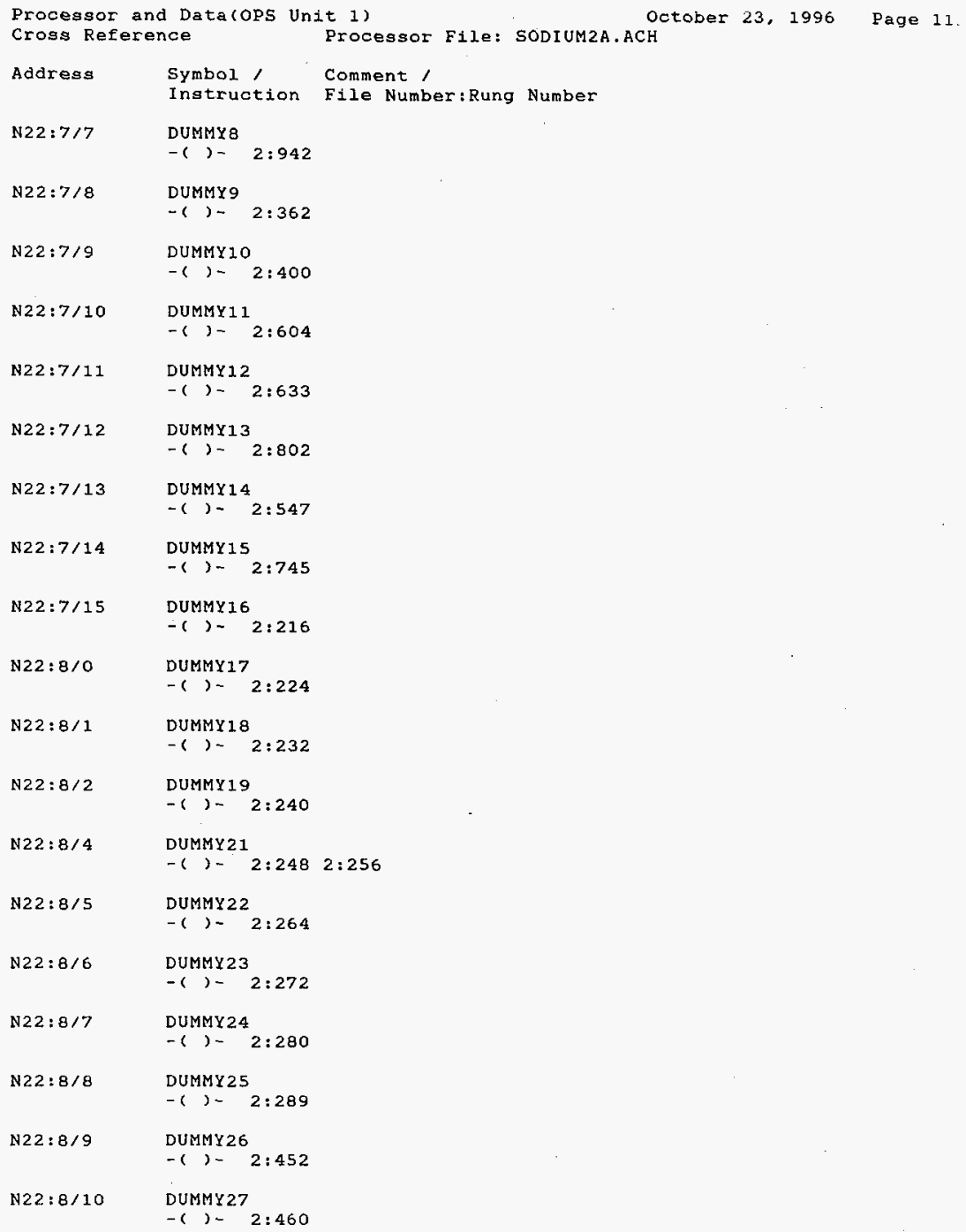




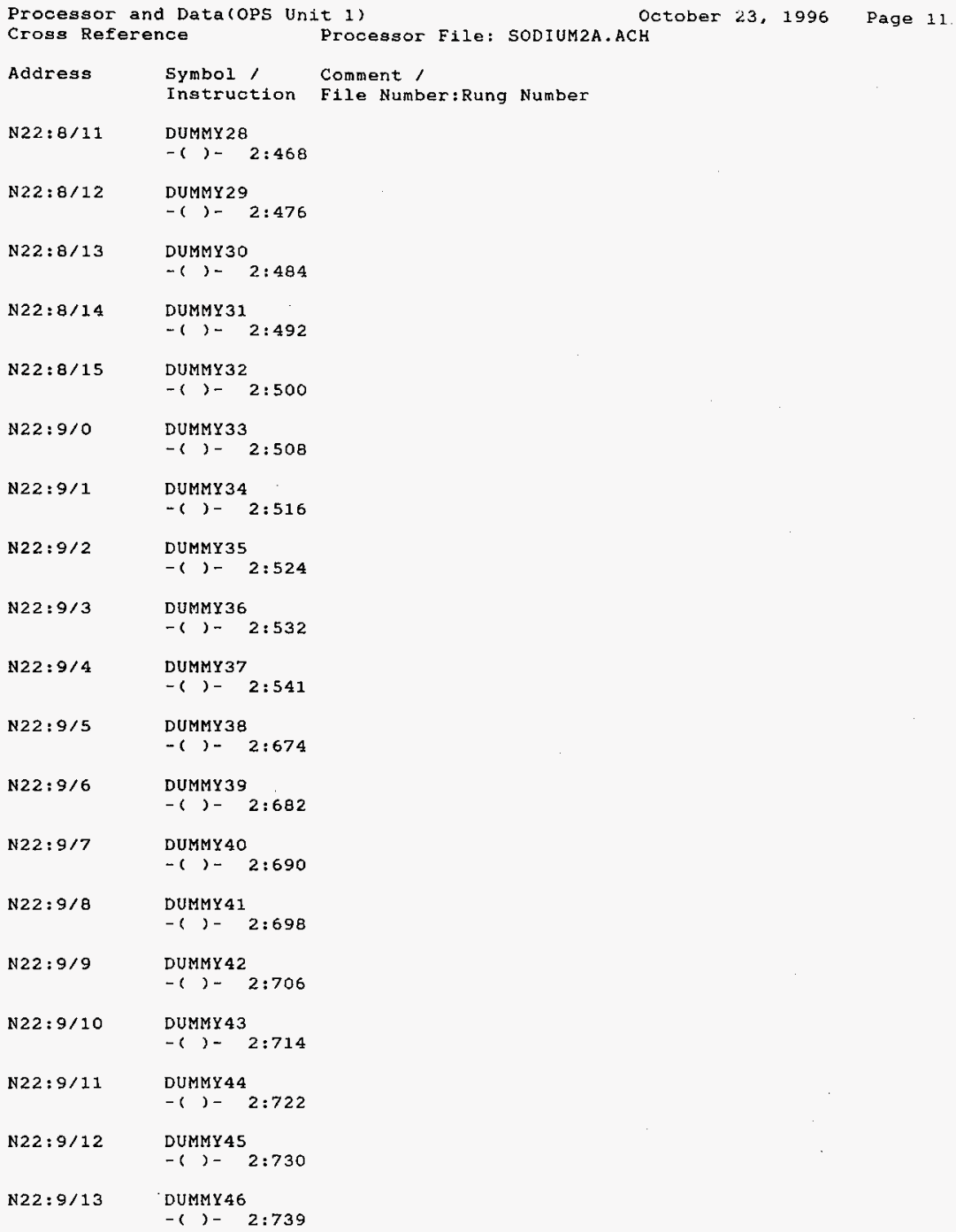

HNF-SD-FF-CSWD-60Rev. 0 


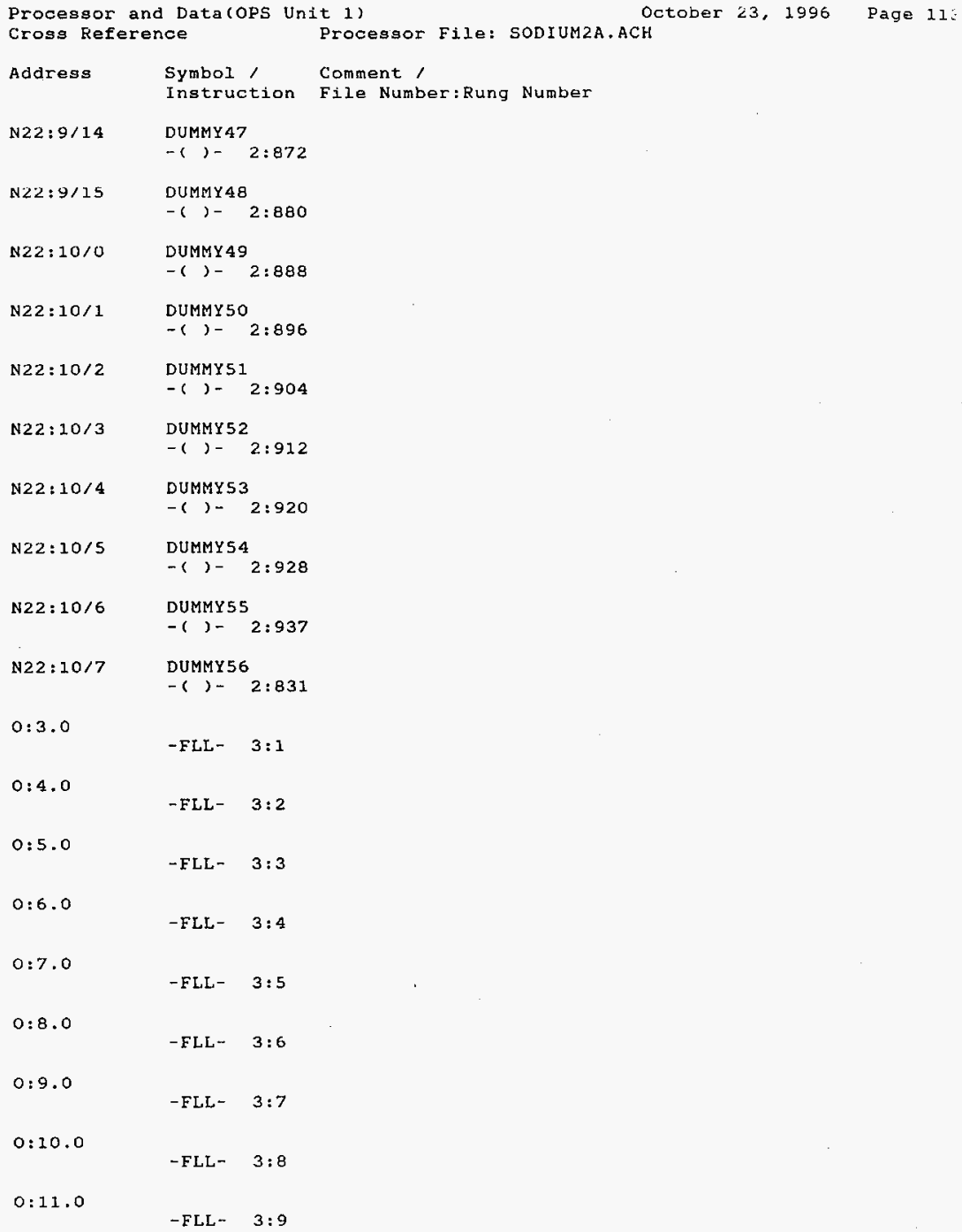




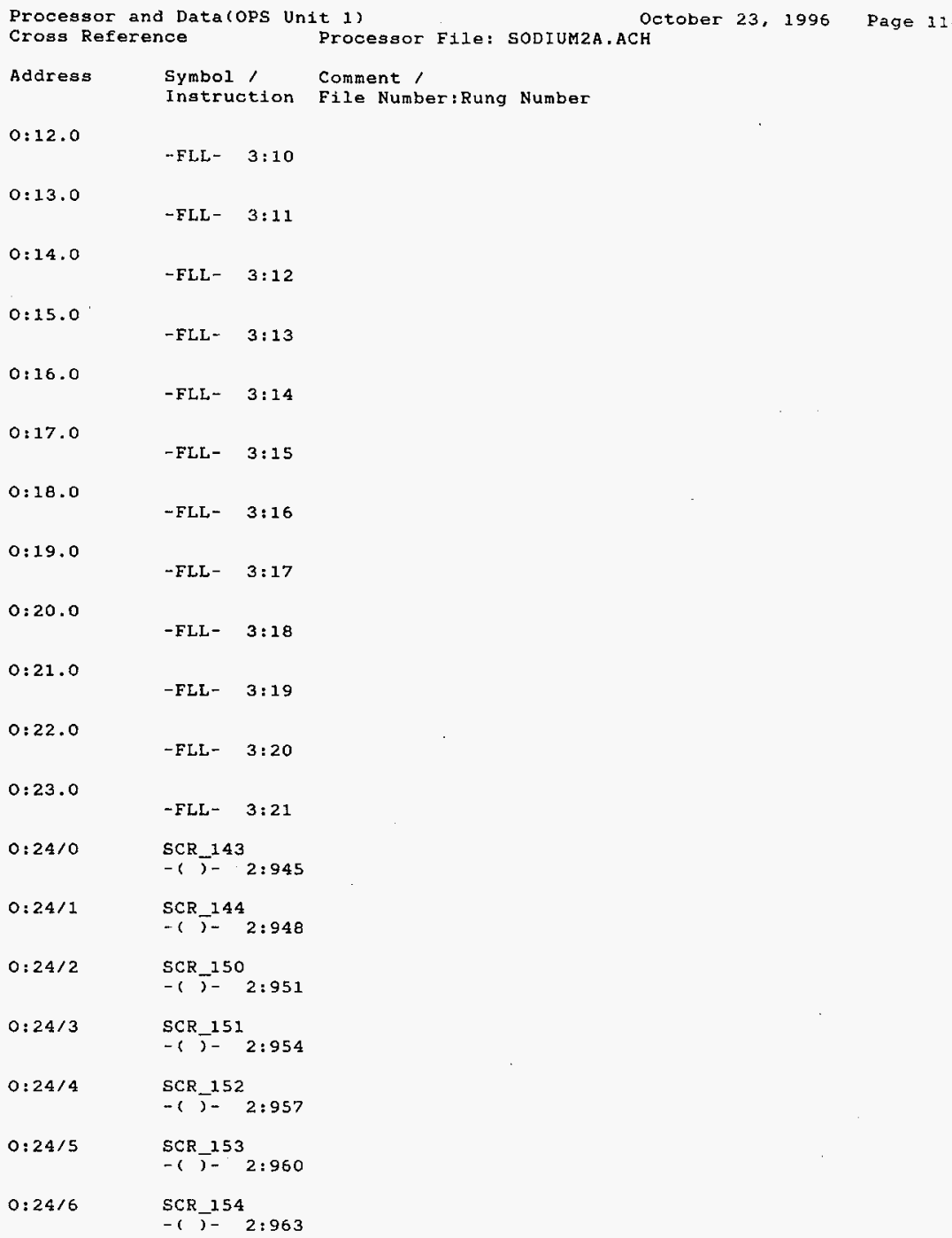

HNF-SD-FF-CSWD-6D Rev. 0 


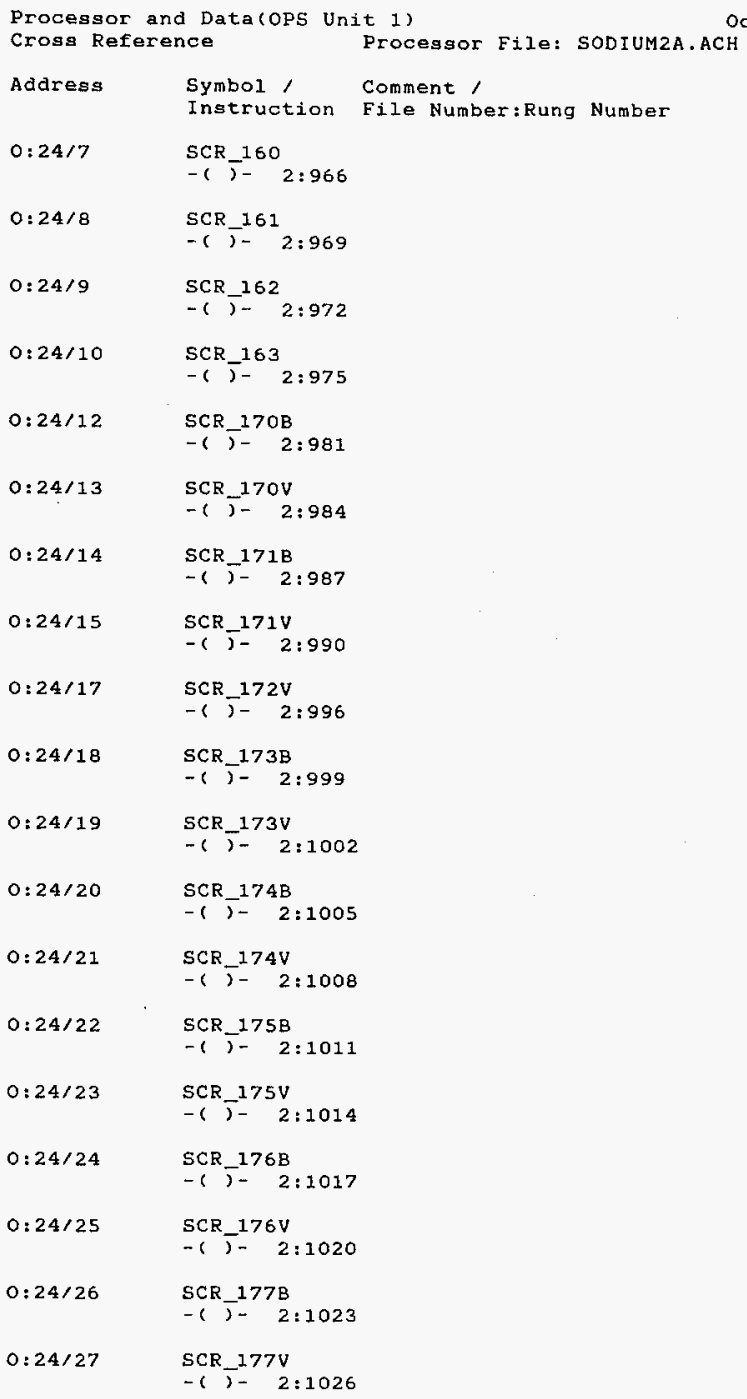

Address

$0: 24 / 7$

$0: 24 / 8$

$0: 24 / 9$

$0: 24 / 10$

$0: 24 / 12$

$0: 24 / 13$

$0: 24 / 14$

$0: 24 / 15$

$0: 24 / 18$

$0: 24 / 19$

$0: 24 / 20$

$0: 24 / 21$

$0: 24 / 22$

$0: 24 / 23$

$0: 24 / 24$

$0: 24 / 25$

$0: 24 / 26$

$0: 24 / 27$

Symbol $/$ Inetruction

SCR 160

( ) $2: 966$

SCR_161

-( )- 2:969

SCR 162

-()$^{-} 2: 972$

SCR_163

- $)$ 2:975

SCR_17OB

$-(\overline{-}-2: 981$

SCR $170 \mathrm{~V}$

$-(\overline{)}-2: 984$

SCR $171 B$

-()$-2: 987$

SCR $171 \mathrm{~V}$

-

SCR_172V

-()$-2: 996$

SCR_173B

$-(\overline{)}-2: 999$

SCR $173 \mathrm{~V}$

- ( ) $2: 1002$

SCR_174B

- () - 2:1005

SCR $174 \mathrm{~V}$

-()$-2: 1008$

SCR_175B

-( ) $-2: 1011$

SCR_175V

$-(\overline{)}-2: 1014$

SCR 1768

- () 2:1017

SCR_176V

$-(\overline{)}-2: 1020$

SCR 177B

$-(j-2: 1023$

SCR_177V

$-(1)-2: 1026$

Comment /

File Number:Rung Number 


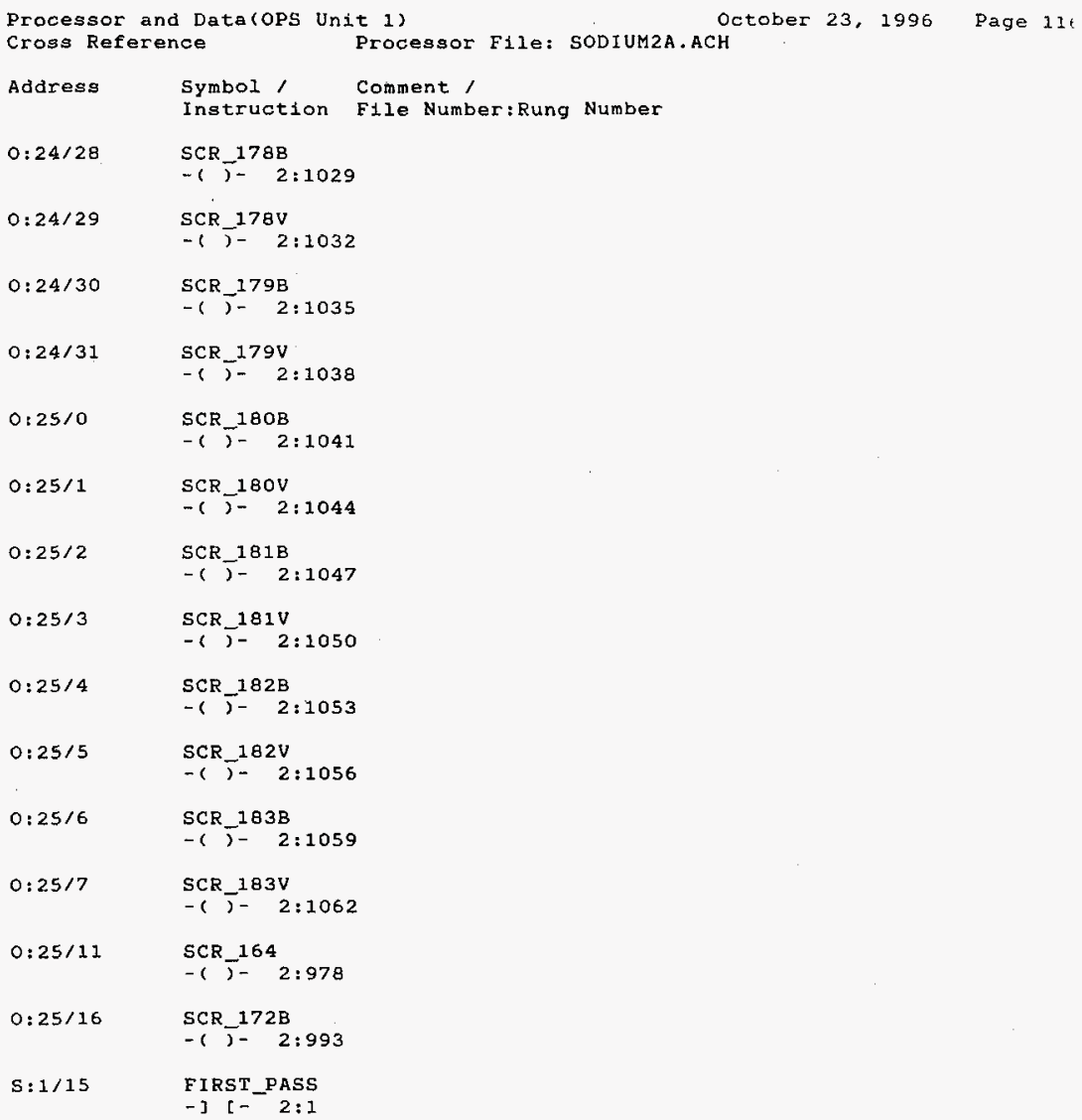

HNF-SD-FF-CSWD- 60 Rev. 0 
Processor and Data(OPS Unit 1)

$T 4: 1$

$T 4: 1 . A C C$

T4: 1/DN

T4: 2

$\mathrm{T} 4: 2, \mathrm{ACC}$

$\mathrm{T} 4: 2 / \mathrm{DN}$

$T 4: 3$

$T 4: 3 / D N$

$\mathrm{T} 4: 4$

T4:4.ACC

$T 4: 5$
TMR 143

-RES- $\quad 2: 944$

-TON- 2:943

-LES- $2: 945$

- ] $[-\quad 2: 944$

TMR144

-RES- 2:947

-TON- $2: 946$

-LES - $2: 948$

- ] $\quad-2: 947$

TMR150

-RES- 2:950

-TON- 2:949

-LES- 2:951

- ] $[-2: 950$

TMR 151

-RES - 2:953

-TON - 2:952

-LES- 2:954

- ] $t-2: 953$

TMR 152

-RES- 2:956

-TON- 2:955 


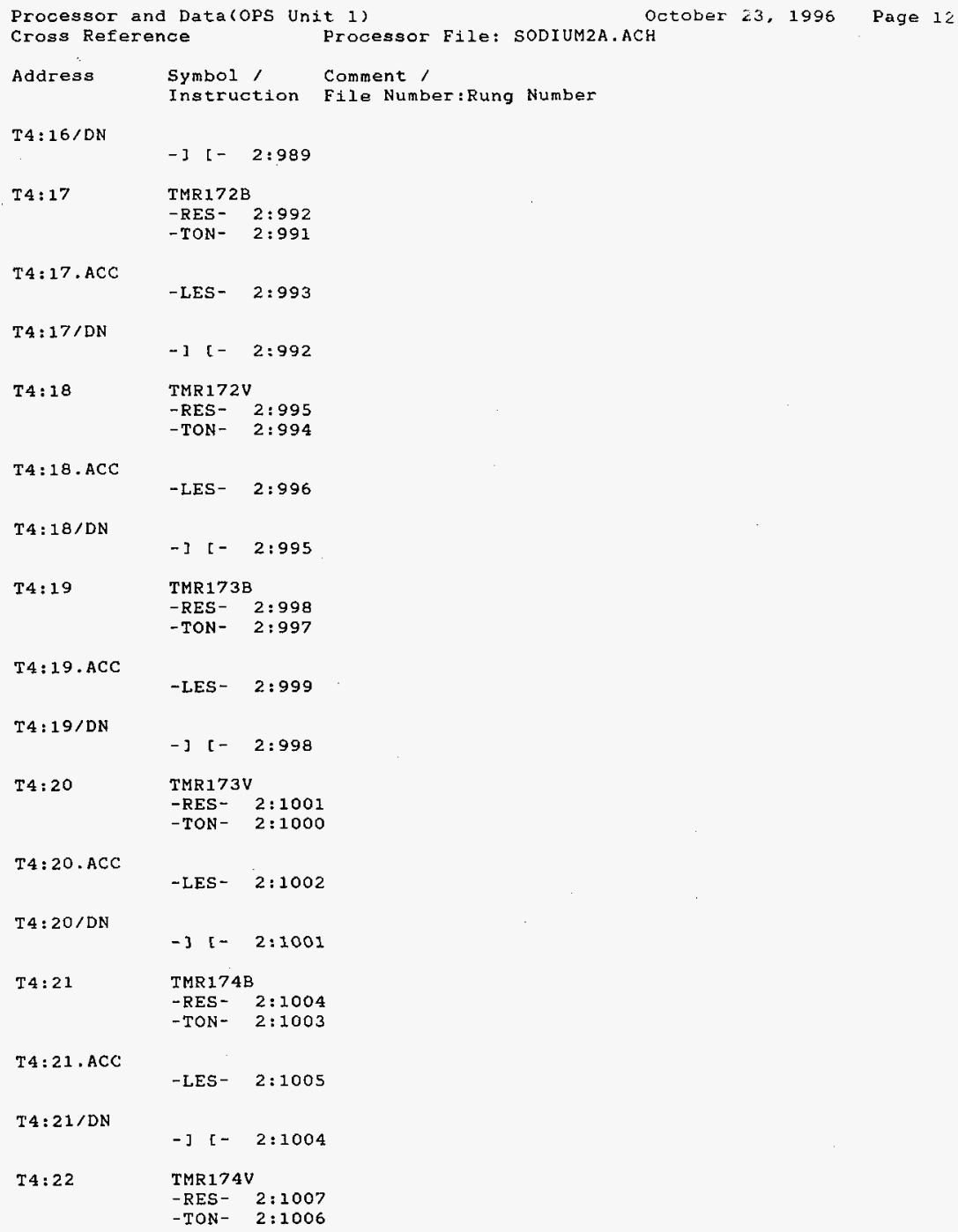

T4:20.ACC

-LES- 2:1002

T $4: 20 / D N$

-3 [ $-2: 1001$

T 4:21

TMR174B

-RES - 2:1004

-TON- 2:1003

T 4:21.ACC

-LES- 2:1005

$\mathrm{T} 4: 21 / \mathrm{DN}$

- ] $[-2: 1004$

T 4: 22

TMR174V

-RES- 2:1007

-TON- 2:1006

HNF-SD-FF-CSWD-60Rev. 0 


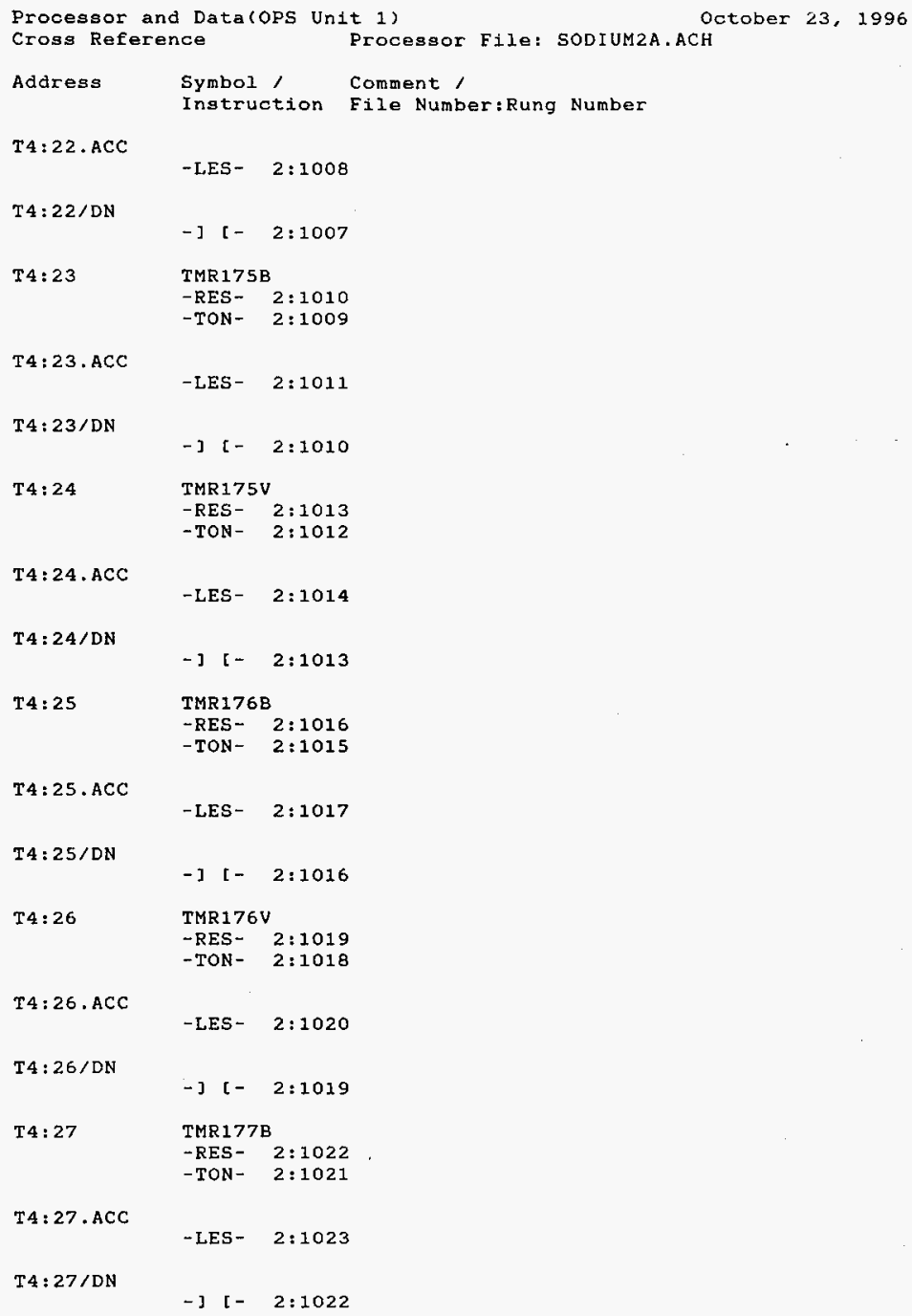

T4:26. ACC

-LES- $2: 1020$

$\mathrm{T} 4: 26 / \mathrm{DN}$

$-][-2: 1019$

T 4: 27

TMR177B

-RES - 2:1022

-TON- 2:1021

T 4: 27. ACC

-LES - 2:1023

$\mathrm{T} 4: 27 / \mathrm{DN}$

- ] [ - 2:1022

HNF-SD-FF-CSWD-60Rev, 0 


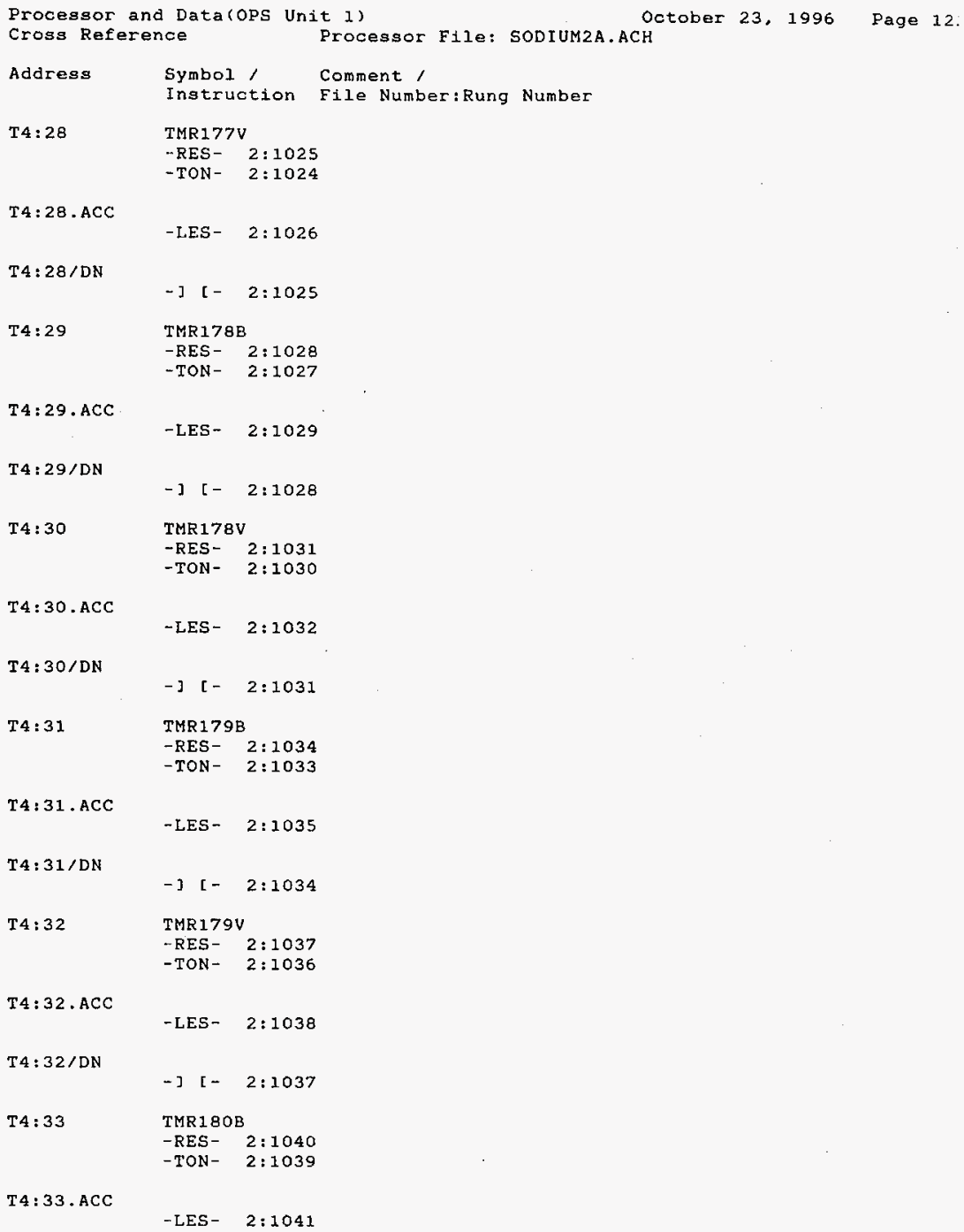

HNF-SD-FF-CSWD-60Rev. 0 


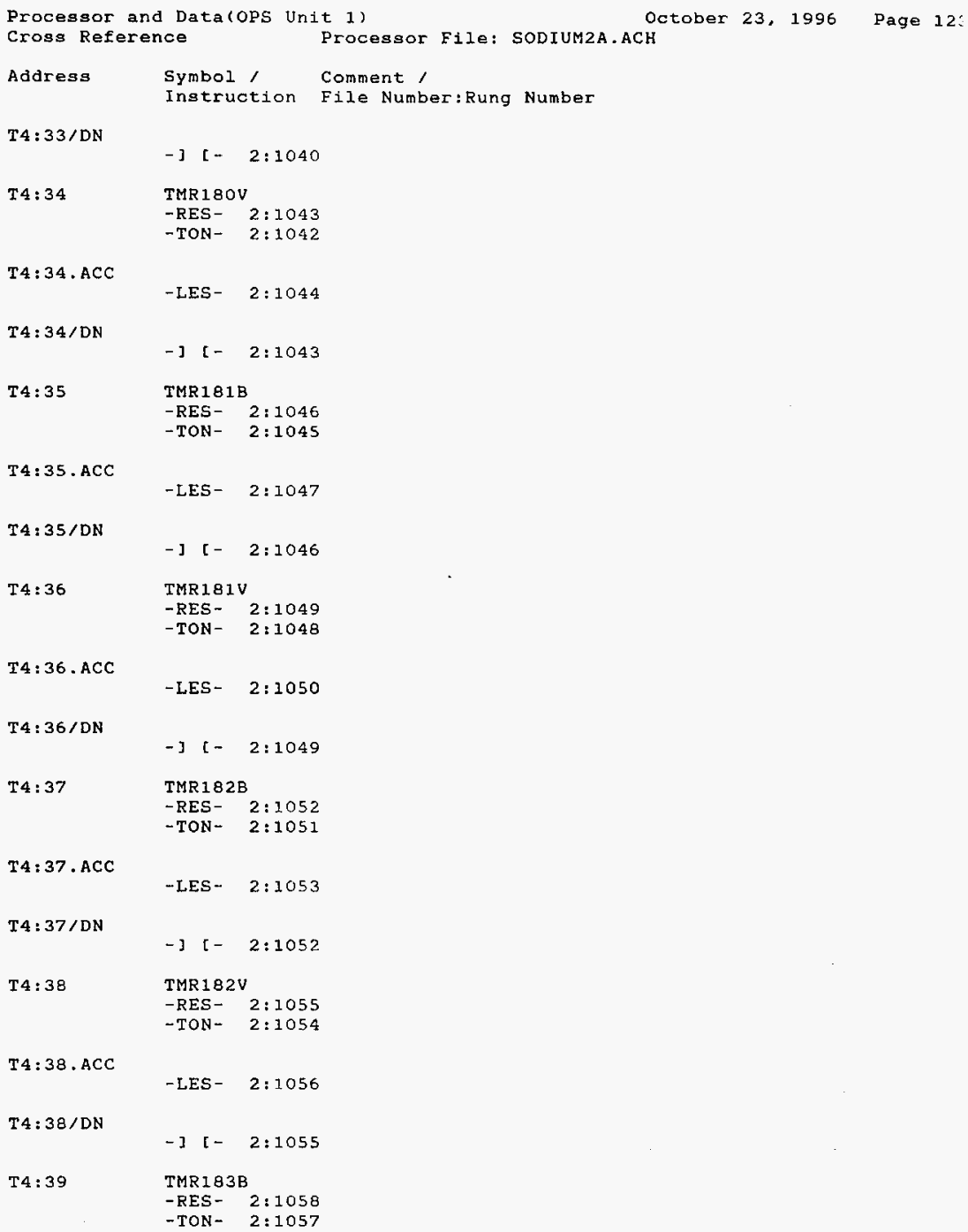




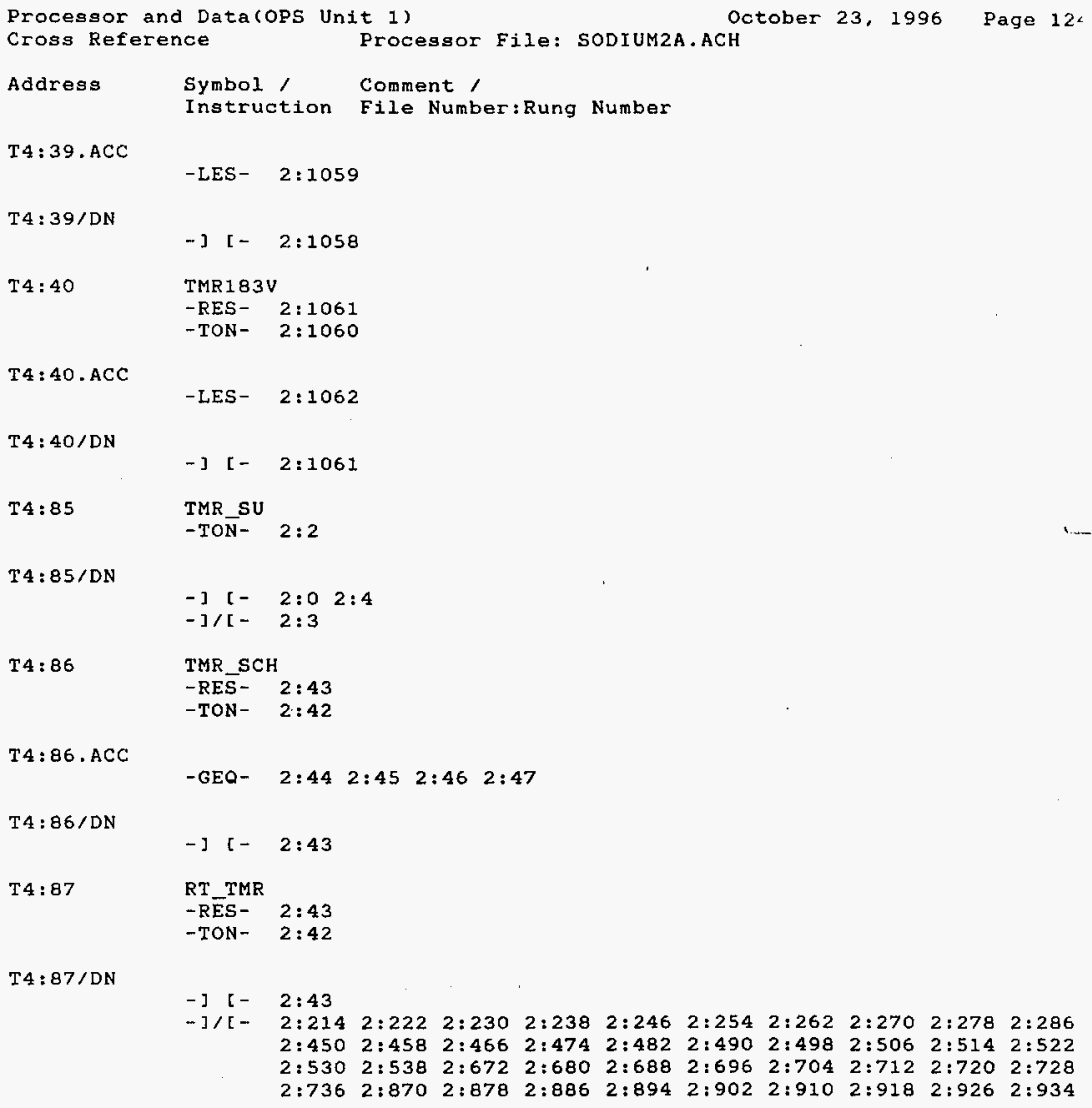

